\title{
Multi-dimensional Nuclear Magnetic Resonance Methods \\ in the Inhomogeneous Magnetic Field
}

\author{
by \\ Lauren M. Burcaw
}
A thesis
submitted to the Victoria University of Wellington in fulfillment of the requirements for the degree of Doctor of Philosophy in Physics.

Victoria University of Wellington 2011 



\begin{abstract}
This thesis introduces new NMR techniques which use the inhomogeneous internal magnetic fields present in the pore space of a porous medium exposed to an external magnetic field to obtain information about the pore size and heterogeneities of the the sample. Typically internal field inhomogeneities are regarded as unwanted due to their effect on various material properties such as relaxation and diffusion. However, in the experiments presented here, we choose samples specifically for their inhomogeneous internal fields and use multi-dimensional NMR methods and simulations to obtain our pore space and heterogeneity information.

We first describe software developed to specifically simulate the internal magnetic field and diffusion through the pore space of a simple sphere pack system. This software generates a sphere pack and calculates the internal magnetic field generated by $z$-aligned magnetic dipoles placed at the center of each sphere. The internal magnetic field gradient is also calculated in the pore space. From there, a random walk method is developed and a realistic reflection off a sphere is introduced. We work through the development of this software and the mathematics behind the algorithms used. This simulation is used in all subsequent experimental chapters.

We then use a two-dimensional exchange experiment to separate the susceptibility induced line broadening with the broadening caused by diffusion through the inhomogeneous field. We observe off-diagonal line broadening as the mixing time increases. We attempt to quantify this off-diagonal growth by selecting points on either side of the off-diagonal maximum and plotting their average as a function of mixing time. A biexponential fit to the average intensities with respect to mixing time results in a characteristic time and from that a characteristic length as a fraction of bead diameter. This experiment is simulated and a biexponential growth is also observed in the simulated off-diagonal
\end{abstract}


with characteristic lengths comparable to experiment.

To obtain a correlation length directly from experiment and not deduce one from a characteristic time, we add a spatial dimension to our exchange experiment in the form of a propagator dimension. This dimension allows us to select 2D spectra based on their $Z$-displacement. We observe off-diagonal growth due to both an increase in $Z$-displacement and an increase in mixing time. We move away from the biexponential fit and move to a relationship based on mixing time, effective diffusion, and $Z$-displacement to directly calculate a characteristic length. We see these same traits in the simulated data which agrees well with experiment.

Lastly, we move away from exchange experiments and move to correlating the transverse relaxation time with the internal field offset. We find that there is correlation at large magnetic field offsets and small $T_{2}$ times which appear to be indicative of sample heterogeneities. To confirm this we use a highly heterogeneous rock core sample which increases the correlations seen at the previous offsets and times. This experiment is more qualitative than the previous two as we do not have a concrete value for the heterogeneity of our samples. The simulation used throughout the thesis, while showing a definite correlation between field offset and $T_{2}$ relaxation, is unable to accurately simulate the experiment and requires more development. 


\section{Acknowledgements}

This thesis would never have happened if it weren't for a conversation between my mother and our then neighbor, Charlie Flaum, about my switching majors from meteorology to physics. From that conversation I got my first internship at Schlumberger-Doll Research in my small hometown of Ridgefield, CT working under Yi-Qiao Song learning NMR. From there I became the "permanent intern" working first under Yi-Qiao and later moving with SDR to Cambridge, MA continuing my NMR education under Bob Kleinberg. To Charlie I am forever grateful for getting me my first research job. I will always remember Yi-Qiao and Bob for their help in teaching me the fundamentals of NMR. Of course I wouldn't be in New Zealand at all if it weren't for Tim Hopper emailing Christoph Arns about an intern at Schlumberger looking to get a PhD outside the US.

I would also like to thank my advisor, Paul Callaghan for his help and support with this thesis throughout the years I've been here. As well as being a remarkable scientist, he is also a remarkable New Zealander and is making a big difference here to do well for his country. I'm pleased that he's fought his cancer well enough to be reading this thesis and I'm sure he's even happier than I am about it. I want to also thank my coadvisor Petrik Galvosas for his help and patience with all my unending questions. I thank Christoph Arns for his insight into how he programs his own simulations and to Greg Peirens for his help with the $900 \mathrm{MHz}$ magnet over at the University of Queensland.

I want to thank everyone in the lab: Kate Washburn, Ben Parkinson, Mark Hunter, Brad Douglass, Meghan Halse, Gui Madelin, Kirk Feindel, Alan Raudsepp, Wilfred Kittler, Jen Brown, Phil Luey, and Matthias Meyer. You all have made my stay here interesting. In particular I want to thank Ben and his wife Becky for occasionally forcing me out of my hermit ways to go out for a few 
drinks, Kirk for always making me laugh, Gui for being himself, Meghan to rant about American politics and teach me to always drink tea out of a ceramic mug, Matthias for all his mathematical help, Mark for his help with all my questions and his delicious homemade bagels, and Jen and her husband Todd for the laughs and various dinners and BBQs which made me feel like I was back at home. Although Kate left the lab only a few days after I arrived, we've since become friends and I'm grateful to her for her and her husband Endre opening their house for Rob and I to stay at in Boston, and for her support through the years. I look forward to seeing many of you upon my return to North America.

Living halfway around the world from old friends is very difficult and I can't find the words to fully express how grateful I am for the friendship of Julie and Ed Pibal during my stay here in New Zealand. They've always made me feel welcome and have really provided me with a home away from home. I will miss them terribly when I leave and I hope they're able to come visit me in the States someday. I will never forget their kindness and generosity, and I congratulate them on the birth of their first child, Victoria.

I'd also like to thank my friends from home who I've been able to keep in touch with for support. In particular I'd like to thank one of my oldest friends, Joe Firrantello for his support for the entire time I've been here when everything is falling to pieces and I've burdened Rob with enough. The Skype drinking sessions were very helpful and we probably should've done them more often even with the terrible time difference. I can't wait to share a pint with him at Zeno's when I'm back and to repay his kindness now that the situation has reversed. I'd also like to thank Kelli McNicholas for making our visits down to Jersey very memorable. The 4 th of July BBQ will probably live in infamy for me. I'm happy to say we will be back in time for her wedding!

I also thank my mom and stepdad for their support in my pursuit of my PhD. I wish my grandma was here to have seen me go to grad school but I know how proud she'd be of me. I also wanted to thank Lynn and Rich Crue for their support and for their blessing in allowing their son to move down here with me. I know it's been very hard on them.

Finally, I dedicate this thesis to my boyfriend, Rob. He quit his job and moved halfway across the world to support me and stayed here for over three 
years dealing with more than his share of stress. His unquestioning willingness to stay by me means more to me than he'll ever know. His support has been utterly invaluable and I do not think I could have completed this thesis without him being here. Our final year in New Zealand marks over nine years together and I cannot believe nine years could go by so quickly. 


\section{Contents}

1 Introduction $\quad 1$

2 Introduction to NMR 5

2.1 Introduction . . . . . . . . . . . . . . . 5

2.2 Nuclear Spin and the Magnetic Moment . . . . . . . . . . . . . . 6

2.3 The Spin Hamiltonian and the Zeeman Interaction . . . . . . . . . 7

2.4 Populations and Coherences . . . . . . . . . . . . . . . 9

2.5 Thermal Equilibrium . . . . . . . . . . . . . . . . . 11

2.6 Classical Description . . . . . . . . . . . . . . . 12

2.7 Signal Detection . . . . . . . . . . . . . . . . . . 15

2.8 Relaxation and the Bloch Equations . . . . . . . . . . . . . . . 15

2.9 Mathematical Tools . . . . . . . . . . . . . . . . . 16

2.9.1 Fourier Transform . . . . . . . . . . . . . . 16

2.9.2 Inverse Laplace Transform . . . . . . . . . . . . . . 17

3 Pulse Sequences $\quad 21$

3.1 Introduction . . . . . . . . . . . . . . . 21

3.2 One Dimensional NMR . . . . . . . . . . . . . . . . . . . . . 21

3.2.1 Free Induction Decay . . . . . . . . . . . . . . . . . 21

3.2.2 Inversion Recovery . . . . . . . . . . . . . . . . 23

3.2 .3 Spin Echo . . . . . . . . . . . . . . . . 24

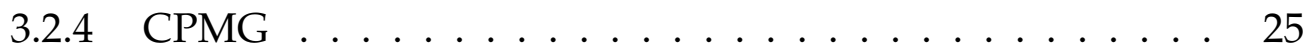

3.2 .5 Stimulated Echo . . . . . . . . . . . . . 25

3.2 .6 PGSE . . . . . . . . . . . . . . 27

3.2 .7 PGSTE ........................ 30 
3.2.8 Bipolar Gradients . . . . . . . . . . . . . . . . . 30

3.2.9 Propagators and q-space . . . . . . . . . . . . . 31

3.3 Two-Dimensional NMR . . . . . . . . . . . . . . 32

3.3.1 Two-Dimensional Exchange Experiments . . . . . . . . . . 33

3.3.2 Two-dimensional Correlation Experiments . . . . . . . . . 35

4 Porous Media $\quad 37$

4.1 Introduction . . . . . . . . . . . . . . . . . . 37

4.2 Properties of Porous Media . . . . . . . . . . . . . . . 37

4.2.1 Porosity and Representative Elementary Volume . . . . . . 38

4.2 .2 Permeability . . . . . . . . . . . . . . . . . . . 39

4.2.3 Formation Factor and Tortuosity . . . . . . . . . . . 40

4.2.4 Wettability . . . . . . . . . . . . . . 41

4.2.5 Surface to Volume Ratio and Surface Relaxivity . . . . . . 42

4.2.6 Pore Size Distribution . . . . . . . . . . . . . . . . . . . 44

4.3 Internal Magnetic Fields . . . . . . . . . . . . . . . . . 44

4.3.1 Internal Magnetic Field Calculations . . . . . . . . . . . . 44

4.3.2 Relaxation Due to Diffusion in Internal Magnetic Fields . . 46

4.3.3 Gradients Due to Internal Magnetic Fields . . . . . . . . . 48

4.4 Diffusion in the Pore Space . . . . . . . . . . . . . . . . . 49

4.4.1 Diffusion in the Short Time Limit . . . . . . . . . . . . . 49

4.4.2 Diffusion in the Long Time Limit . . . . . . . . . . . . 50

4.4.3 Diffusion in the Intermediate Time Limit . . . . . . . . 52

4.4.4 Diffusive Diffraction . . . . . . . . . . . . . . 53

4.5 This Thesis . . . . . . . . . . . . . . . . 53

5 Simulation $\quad 57$

5.1 Introduction . . . . . . . . . . . . . . . . 57

5.2 Creating the Bead Pack . . . . . . . . . . . . . . . 58

5.3 Simulating the Internal Magnetic Field . . . . . . . . . . . . . 58

5.4 Simulating the Magnetic Field Gradient . . . . . . . . . . . . . 63

5.5 Random Walk Along $x y z$-axes . . . . . . . . . . . . . . . . . . 65

5.6 Random Walk with Random Angle . . . . . . . . . . . . . . . . . 66 
5.6.1 Reflection Off A Circle . . . . . . . . . . . . . 68

5.6 .2 Reflection off a Sphere . . . . . . . . . . . 73

5.7 Adding Relaxation . . . . . . . . . . . . . . . . 78

5.8 Creating Simulated 2D Spectra $\ldots \ldots \ldots$. . . . . . . . . . 82

5.9 Conclusions ......................... 83

6 Frequency Exchange $\quad 85$

6.1 Introduction . . . . . . . . . . . . . . 85

6.2 Method . . . . . . . . . . . . . . . . . 87

6.3 Experimental . . . . . . . . . . . . . . . . 88

6.4 Results and Discussion . . . . . . . . . . . . . . . . . 89

6.4.1 100 micron Bead Pack . . . . . . . . . . . . . 89

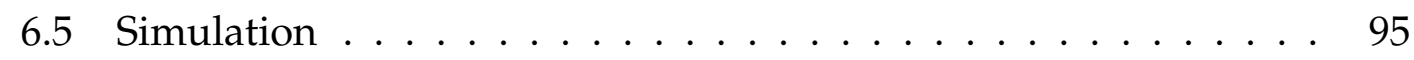

6.5.1 10 micron Bead Pack . . . . . . . . . . . . . . 97

6.6 Conclusion . . . . . . . . . . . . . . . . . . 103

7 Propagator Resolved Frequency Exchange 105

7.1 Introduction . . . . . . . . . . . . . . . . . . . 105

7.2 Method ........................ 106

7.2.1 Experimental . . . . . . . . . . . . . 106

7.2.2 Pulse Sequence . . . . . . . . . . . . . . . . 107

7.3 Experimental and Simulation Results . . . . . . . . . . . . . . 109

7.4 Theory . . . . . . . . . . . . . . . . . . 117

7.5 Conclusions . . . . . . . . . . . . . . . . 120

$8 \quad T_{2}$ - $B_{z}^{i}$ Correlation $\quad 123$

8.1 Introduction . . . . . . . . . . . . . . . . . . 123

8.2 Simulating the $|g|-B_{z}^{i}$ Correlation . . . . . . . . . . . . . . 124

8.3 Summary of $|g|-B_{z}^{i}$ Correlation Spectra . . . . . . . . . . . . . . . . 129

8.4 Pulse Sequence . . . . . . . . . . . . . . . . . . . . . . . 133

8.5 Relaxation in the Inhomogeneous Magnetic Field . . . . . . . . . 134

8.6 Analysis of FT-ILT Data . . . . . . . . . . . . . . . . 135

8.6.1 Generating the Test Spectrum . . . . . . . . . . . 136 
8.6.2 Creating and Processing the Data Matrix from the Test Spectra . . . . . . . . . . . . . . . . 139

$8.7 T_{2}-B_{z}^{i}$ Correlation for a Monodisperse Glass Bead Pack . . . . . 141

$8.8 T_{2}^{\prime}-B_{z}^{i}$ Correlation for a Bentheimer Sandstone Core $\ldots \ldots \ldots$

8.9 Simulating the $T_{2}-B_{z}^{i}$ Correlation in the Bead Pack . . . . . . 146

8.10 Conclusions . . . . . . . . . . . . . . . . . . . . . . . . 149

9 Summary and Future Work 151

9.1 Future Work . . . . . . . . . . . . . . . . . . . . . . . . . 153

9.2 Conclusion . . . . . . . . . . . . . . . . . . . . . . 154

A Publications from this Thesis 155 


\section{Chapter 1}

\section{Introduction}

The study of porous materials using nuclear magnetic resonance (NMR) has always been of interest due to the non-invasiveness NMR affords. A porous medium is anything which contains void spaces, or pores within the material. Examples include such wide-ranging materials as breads, sponges, bones, and rocks, and can range in size from zeolites to aquifers. Porous media is of particular interest in the oil industry, since oil reservoirs are comprised of rock with oil in the pore space.

When a porous material is placed in an external magnetic field, such as that found in an NMR machine, an inhomogeneous magnetic field arises in the pore space of the sample. The strength of this field is dependent on a property of the material known as the magnetic susceptibility, which we will discuss in more detail in chapter 4 . In this thesis, we seek to exploit the inhomogeneous magnetic field to characterize the pore space of the material. To do this, we will use two samples of monodisperse glass beads of two different bead diameters, and a sandstone rock core.

The thesis is split into two major parts. The first part is made up of three chapters which will be comprised of a review of NMR and porous media. First we will introduce what NMR is and the theory behind the phenomenon. Then we will review some of the major pulse sequences used to measure properties such as the spin-spin and transverse relaxation times and diffusion. Then we will introduce how these properties change when inside the boundaries of a 
pore.

The second part of the thesis will describe the development of a simulation and the three internal magnetic field experiments. First, we will summarize the creation of random walk program used to generate a random pack of spheres and simulate the inhomogeneous internal magnetic field, diffusion through the pore space, and transverse relaxation. Then we discuss a two-dimensional exchange experiment where we use the line broadening due to diffusion through the internal field to characterize the pore space of our two monodisperse glass bead samples. From there we move on to include a propagator dimension to this exchange experiment which will allow us to separate the exchange spectra according to the $Z$-displacement of the diffusing spins in the pore space. Finally we describe a correlation experiment where we correlate transverse relaxation with the inhomogeneous internal magnetic field.

This thesis is comprised of 9 chapters, including this introductory chapter. Short summaries of the other chapters are as follows:

In chapter 2 we provide an overview of nuclear magnetic resonance in which we give both a classical and quantum mechanical description of magnetic resonance and review some basic tenets needed to fully understand this thesis. We first start with introducing the nuclear spin and magnetic moment, and continue on to a quantum mechanical description, and then a classical description. From there we describe how we detect a signal and review spin-spin and transverse relaxation. Finally, we describe two major mathematical tools used in this thesis, the Fourier transform and the inverse Laplace transform.

Chapter 3 includes descriptions of some basic fundamental pulse sequences frequently used in NMR, and is split into two major parts: one-dimensional pulse sequences and two-dimensional pulse sequences. The one-dimensional section will cover the major 1D sequences such as the free induction decay, inversion recovery, spin and stimulated echoes, the Carl-Purcell-Meiboom-Gill method and the use of pulsed field gradients. The two-dimensional section will introduce exchange and correlation experiments.

Porous media and the important aspects of porous media and NMR are summarized in chapter 4 . We first discuss the major properties of porous media such as porosity, permeability, wettability, the surface to volume ratio, and the sur- 
face relaxivity. We discuss the properties of internal magnetic fields and internal field gradients in the pore space and discuss how this effects relaxation in the pore space. Finally we review restricted diffusion and how diffusion in porous media varies from free diffusion.

Chapter 5 reviews the development of the Monte Carlo simulation used for the subsequent chapters. We first discuss the creation of the bead pack and the generation of the internal magnetic field and internal magnetic field gradient in the pore space of this bead pack. The drawbacks to a random walk along the $x y z$-axis are discussed and a random walk which steps along a varying angle is introduced. We then go into the mathematics of reflecting a tracer point off a circle and apply a similar method to reflection off a sphere. Finally we add relaxation to the simulation and describe how we generate two-dimensional spectra.

We present our results for a two-dimensional NMR experiment in chapter 6 in which we describe a method to observe water molecules diffusing through the pore space of a monodisperse bead pack. We use two different samples of monodisperse beads and run the experiment at two different field strengths of $400 \mathrm{MHz}$ and $900 \mathrm{MHz}$. Finally we simulate the results of the larger diameter bead pack.

We expand the experiment from the previous chapter and in chapter 7 we include a propagator dimension so as to add a spatial dimension. The addition of a spatial dimension allows us to obtain 2D exchange spectra that are defined both by mixing time and displacement. In this chapter we present the results of this new experiment using a single monodisperse bead pack and field strength of $400 \mathrm{MHz}$. We then again use our program to simulate the results of this experiment, including both the propagators and the 2D spectra.

In our final experimental chapter, chapter 8 , we use a correlation experiment to correlate the transverse relaxation time with the internal magnetic field. For this experiment we again use the same monodisperse bead pack as in the previous chapter, and also use a sandstone rock core. As for the previous two chapters, we simulate the results of the experiment, but due to the limitations of our software, we are unable to get an exact representation of experiment.

We conclude the thesis in chapter 9 with a short summary of the results obtained and include possibilities for future continuation of the research. 


\section{Chapter 2}

\section{Introduction to NMR}

\subsection{Introduction}

Nuclear magnetic resonance was discovered by F. Bloch [1] and E.M. Purcell [2] in 1946. For their discovery, they were jointly awarded the Nobel Prize for physics in 1952. In the beginning, NMR was dominated by the traditional methods of spectroscopy, and utilized a slow field sweep to measure the sample's spectrum, known as a "continuous wave" spectrum. The use of short frequency pulses to excite the sample were relegated mainly to relaxation measurements.

In the 1970s, the development of Fourier spectroscopy and two-dimensional spectroscopy by Ernst earned him the Nobel Prize in chemistry in 1991. These developments allowed spectra to be measured in a shorter period of time using short radio frequency pulses. The introduction of inexpensive computers in the late-1960s made Fourier spectroscopy practical and today this type of spectroscopy has replaced nearly all continuous wave spectrometers. The addition of a second dimension in NMR measurements allowed more information to be obtained from samples.

NMR is particularly useful for researching porous media. As we are most interested in the fluid within the pores of the medium, be it hydrocarbon, water, or a mixture of both, NMR is a noninvasive method to study this fluid. Properties of the resultant NMR signal can give us information about the pore space such as pore size, pore composition, and the structure of the sample. There 
are various ways of processing the signal including the Fourier transform and Inverse Laplace transform. The development of new NMR methods to study porous media is valuable to many industries including the oil industry, biology, and chemical engineering.

We begin this thesis with an introduction describing how NMR works using both a quantum mechanical description and a semi-classical description. There are many sources for this information, however we have found Callaghan [3, 4], Levitt [5], Keeler [6], and Becker [7] particularly useful.

\subsection{Nuclear Spin and the Magnetic Moment}

Atoms posses two types of angular momentum, orbital angular momentum which arises from the motion of the particle and is dependent on the particle's history, and spin, $I$, which is unrelated to the motion of the particle or its history. Quantum orbital angular momentum can be analogous to its classical counterpart, but it's important to note that unlike classical spin, quantum spin is not caused by the rotation of the particle but is rather an intrinsic property of the particle itself. Atomic nuclei have a characteristic spin which may have either integer or half integer values ranging from $-I$ to $I$. For example, the spin of a hydrogen proton, ${ }^{1} H$ is $\frac{1}{2}$ while the spin of a deuterium proton, ${ }^{2} H$ is 1 . For this thesis, we will focus mostly on spin- $\frac{1}{2}$ as our experiments involve only ${ }^{1} H$.

A spin- $\frac{1}{2}$ particle will have $I$ values of $\pm \frac{1}{2}$, which can also be written as $\uparrow$ for the "spin up" state, $\frac{1}{2}$ and $\downarrow$ for the "spin down" state, $-\frac{1}{2}$. A single hydrogen proton will have two spin states, either spin up or spin down, whereas a pair of hydrogen protons can have a combination of four spin states: $\uparrow \uparrow, \uparrow \downarrow, \downarrow \uparrow$, and $\downarrow \downarrow$. Each of these states will correspond to an energy level, where the ground state is the state which possesses the lowest energy.

Any particle which possesses spin will also have a magnetic dipole moment, $\mu$, which is proportional to the particle's spin by a factor $\gamma$, known as the gyromagnetic ratio. If this magnetic dipole moment is exposed to an external magnetic field, it will experience a torque, $\mu \times B$, and precess about the field. A positive $\gamma$ results in $\mu$ aligning in the same direction as $I$, whereas a negative $\gamma$ 
results in $\mu$ aligning antiparallel to $I$. However, it is important to note that the spin polarization itself does not align with the field but rather precesses about the field at a frequency $\omega_{0}$, known as the Larmor frequency. The Larmor frequency is given by

$$
\omega_{0}=\gamma B_{0}
$$

where $B_{0}$ is the external magnetic field at the site of the spin system. The direction of precession is given by the sign of $\gamma$ where a negative gamma will result in a counterclockwise precession and a positive gamma will result in a clockwise precession as shown in figure 2.1.
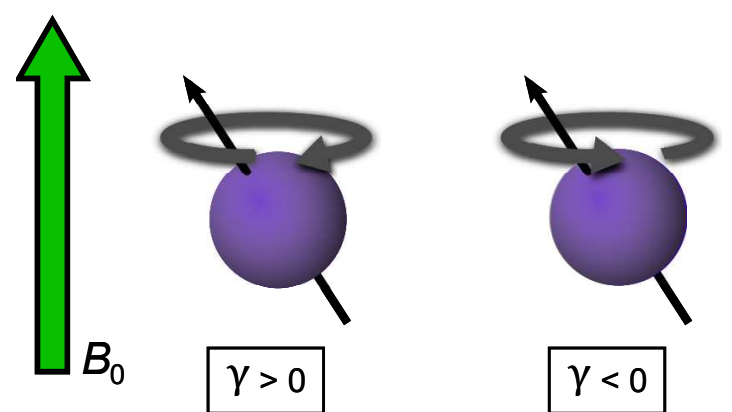

Figure 2.1: Spin precession in an external magnetic field, $B_{0}$. A positive gyromagnetic ratio, $\gamma$ causes the magnetic moment, $\mu$ to precess clockwise about $B_{0}$. If $\gamma<0$, $\mu$ will precess counterclockwise.

Placing a proton $\left(\gamma=2.675 \times 10^{8} \mathrm{rad} \mathrm{s}^{-1} \mathrm{~T}^{-1}\right)$ in a laboratory magnet with a magnetic field strength, $B_{0}$ of $9.4 T$ will result in a cyclic Larmor frequency $\left(\frac{\omega_{0}}{2 \pi}\right)$ of $400 \mathrm{MHz}$.

\subsection{The Spin Hamiltonian and the Zeeman Interac- tion}

A spin- $\frac{1}{2}$ particle has two eigenstates, $|m\rangle$, given by $\left|+\frac{1}{2}\right\rangle$ or $\left|-\frac{1}{2}\right\rangle$, which we write as $|\alpha\rangle$ and $|\beta\rangle$ respectively. A spin in state $|\alpha\rangle$ is polarized along the $+z$-axis and a spin in state $|\beta\rangle$ is polarized along the - $z$-axis. 
The state of a particle, $|\Psi\rangle$ is given by

$$
|\Psi\rangle=\sum_{m} a_{m}|m\rangle
$$

so a spin- $\frac{1}{2}$ particle will have the state

$$
|\Psi\rangle=a_{\alpha}|\alpha\rangle+a_{\beta}|\beta\rangle
$$

where $a_{\alpha}$ and $a_{\beta}$ are the complex amplitudes of each eigenstate.

To learn about the dynamics of the spin system we use the Schrödinger equation

$$
i \hbar \frac{\partial}{\partial t}|\Psi(t)\rangle=\hat{H}|\Psi(0)\rangle
$$

where $\hat{H}$ is the Hamiltonian operator, and $\Psi(t)$ is the state of the particle at time $t$, given by equation 2.2. Using this equation with knowledge of the Hamiltonian operator and the initial state $\Psi(0)$ allows us to predict future states of the particle. When $B_{0}$ is orientated along the $z$-axis, the Hamiltonian has the form

$$
\hat{H}=-\gamma \hbar B_{0} I_{z}
$$

which is known as the Zeeman Hamiltonian.

Outside an external magnetic field, a spin is $(2 I+1)$-fold degenerate, meaning that a value of $I=\frac{1}{2}$ will have two substates of the same energy, and a value of $I=1$ will have three substates of the same energy. To break this degeneracy, we can place the spin system inside a magnetic field. In an external magnetic field, the spin- $\frac{1}{2}$ particle will split into two energy levels as seen in figure 2.2a, and the spin- 1 particle will split into 3 energy levels as seen in figure $2.2 \mathrm{~b}$. No matter the value of $I$ the energy level separation is given by the Zeeman Hamiltonian as $-\gamma \hbar B_{0}$. This is known as Zeeman splitting.

Suppose we know the state of a spin in an external field $B_{0}$ at time $t=0$ and wish to know the state of a spin at some other time $t$. If $\hat{H}$ is constant with time, we can find the state $\Psi(t)$ from equation 2.4

$$
|\Psi(t)\rangle=\hat{U}(t)|\Psi(0)\rangle
$$


a).

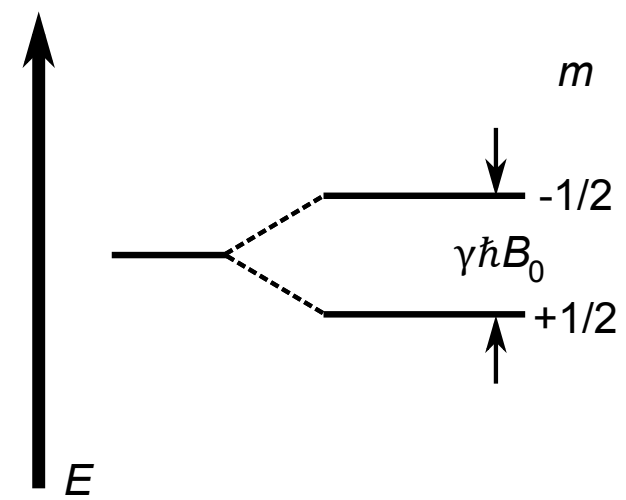

b).

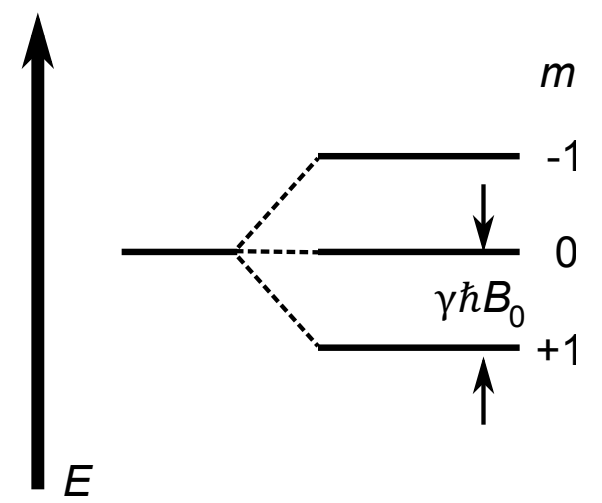

Figure 2.2: a). Zeeman splitting of an $I=\frac{1}{2}$ spin. Upon exposure to an external magnetic field, $B_{0}$, the particle splits into two energy levels separated by $E=\gamma \hbar B_{0}$. b). Zeeman splitting of an $I=1$ particle which when exposed to an external magnetic field splits into three energy levels separated by $E=\gamma \hbar B_{0}$.

where $\hat{U}(t)$ is the evolution operator given by

$$
\hat{U}(t)=\exp (-i \hat{H} t / \hbar)
$$

Combining equations 2.5 and 2.7 we have an evolution operator $U(t)=$ $\exp \left(i \gamma B_{0} I_{z} t\right)$ which is equivalent to a clockwise rotation about the $z$-axis at an angle $\gamma B_{0} t$. From equation 2.1, this translates to a precession about the $z$-axis at the Larmor frequency, $\omega_{0}$.

\subsection{Populations and Coherences}

So far we've described what happens to a single spin, however, as stated before, in NMR we are concerned with what happens in a typical sample where we have large ensemble of spins in a variety of quantum states $|\Psi\rangle$. We can break this ensemble up into subensembles of equal quantum states $|\Psi\rangle$, where each ensemble has a probability $p_{\Psi}$. We define the operator $\hat{\rho}$ as the density matrix to generalize the method of ensemble averaging. $\hat{\rho}$ can be written mathematically as 


$$
\hat{\rho}=\sum_{\Psi} p_{\Psi}|\Psi\rangle\langle\Psi|
$$

The spin density operator can be thought of as the quantum mechanical equivalent to the classical mechanical density in that it describes the number of points in phase space. However it has nothing to do with the physical density of the sample. We can use $\hat{\rho}$ to describe the full state of the system rather than having to define each subensembles' quantum state. Thus if we want to make a measurement on the system, we only need two spin operators, $\hat{\rho}$ and the spin operator for the measured observable.

The evolution of $\hat{\rho}$ is given by the Liouville equation which is found by simply using the Schrödinger equation

$$
i \frac{\delta \hat{\rho}}{\delta t}=[\hat{H}, \hat{\rho}]
$$

where $[\hat{H}, \hat{\rho}]$ is the commutator given by $[\hat{H}, \hat{\rho}]=\hat{H} \hat{\rho}-\hat{\rho} \hat{H}$.

For an ensemble of spin-1/2, the matrix representation of $\hat{\rho}$ is

$$
\hat{\rho}=\left(\begin{array}{cc}
\rho_{\alpha \alpha} & \rho_{\alpha \beta} \\
\rho_{\beta \alpha} & \rho_{\beta \beta}
\end{array}\right)=\left(\begin{array}{ll}
\overline{a_{\alpha} a_{\alpha} *} & \overline{a_{\alpha} a_{\beta} *} \\
\overline{a_{\beta} a_{\alpha} *} & \overline{a_{\beta} a_{\beta} *}
\end{array}\right)
$$

where the diagonal components $\left(\rho_{\alpha \alpha}\right.$ and $\left.\rho_{\beta \beta}\right)$ are called the populations of states $|\alpha\rangle$ and $|\beta\rangle$, and the off-diagonal components $\left(\rho_{\alpha \beta}\right.$ and $\left.\rho_{\beta \alpha}\right)$ are called the coherences between states $|\alpha\rangle$ and $|\beta\rangle$. Since the state of each spin is normalized, the sum of the two populations must be unity. The difference between the two populations indicates the net longitudinal spin polarization, or magnetization in the sample.

A state where the population in $|\alpha\rangle$ is greater than that in state $|\beta\rangle$ means that the net magnetization is parallel to the external field, a state which has a greater population in the $|\beta\rangle$ state means that the net magnetization is antiparallel to the external field. If the population in the two states is equal, that means there is no net magnetization the direction of the external field in the sample.

The coherences give us information about the net transverse polarization to the external field, that is to say the net spin polarization perpendicular to the external field. $\rho_{\alpha \beta}$ and $\rho_{\beta \alpha}$ are complex numbers, which means they have both 
an amplitude and a phase. Physically we can picture this as a vector in the transverse, or $x y$-plane, where the phase indicates the direction of the vector, while the amplitude determines the vector's magnitude.

\subsection{Thermal Equilibrium}

If we want to take a measurement of $I_{z}$ in the sample, we begin with the equation for the expectation value, found by calculating $\left\langle m\left|I_{z}\right| m\right\rangle$, so the expectation value of $I_{z}$ in a state $|\Psi\rangle$ given by equation 2.3 is

$$
\left\langle\Psi\left|I_{z}\right| \Psi\right\rangle=\alpha\left|a_{\alpha}\right|^{2}+\beta\left|a_{\beta}\right|^{2} .
$$

To measure an ensemble of spins in a sample we expand this equation to include all the subensembles

$$
\overline{\left\langle\Psi\left|\hat{I}_{z}\right| \Psi\right\rangle}=\sum_{\Psi} p_{\Psi}\left\langle\Psi\left|\hat{I}_{z}\right| \Psi\right\rangle
$$

For a sample of spin- $\frac{1}{2}$ particles in an NMR machine, we know that the spins will split into two eigenstates $|\alpha\rangle$ and $|\beta\rangle$ as given by the Zeeman Hamiltonian, equation 2.5. Using equations 2.2, 2.11, and 2.12 we have

$$
\overline{\left\langle\Psi\left|\hat{I}_{z}\right| \Psi\right\rangle}=\frac{1}{2}\left(\overline{\left|a_{\alpha}\right|^{2}}-\overline{\left|a_{\beta}\right|^{2}}\right) .
$$

What this equation tells us is that the average $I_{z}$ depends on the difference in population of the two different energy levels. As first mentioned in section 2.4, this difference in population gives us information about the polarization of the sample.

If we allow the $N$ spins in the system to reach thermal equilibrium, the population in each energy level is given by the Boltzmann distribution [8]:

$$
\frac{N_{i}}{N}=\frac{\exp -E_{i} / k_{B} T}{\sum_{i} \exp -E_{i} / k_{B} T}
$$

where $N_{i} / N$ is the number of spins in state $i, E_{i}$ is the energy of state $i, k_{B}=$ $1.381 \times 10^{-13} \mathrm{JK}^{-1}$ is the Boltzmann constant, and $T$ is the temperature measured in K. For a spin- $\frac{1}{2}$ system, we know that there will be two energy levels 
whose energy is given from the Zeeman splitting as $-\gamma \hbar B_{0}$. Using equation 2.1 and 2.14 we calculate the Bolzmann distribution for a spin- $\frac{1}{2}$ system:

$$
\overline{\left|a_{ \pm \frac{1}{2}}\right|^{2}}=\frac{\exp \left( \pm \hbar \omega_{0} / 2 k_{B} T\right)}{\exp \left(-\hbar \omega_{0} / 2 k_{B} T\right)+\exp \left(\hbar \omega_{0} / 2 k_{B} T\right)} .
$$

The difference between the two populations depends on energy level difference $\hbar \omega_{0}$ and the Boltzmann energy $k_{B} T$. At room temperature the difference between $\hbar \omega_{0}$ and $k_{B} T$ is on the order of five magnititudes. This small difference allows us to simplify equation 2.15 to

$$
\overline{\left|a_{ \pm \frac{1}{2}}\right|^{2}}=\frac{1}{2}\left[1 \pm \hbar \omega_{0} / 2 k_{B} T\right] .
$$

The Boltzmann equation tells us that the lower energy state is more populated than the higher energy state. This can be seen in figure 2.3, where we have an ensemble of spins in thermal equilibrium in an external magnetic field, $B_{0}$, which is aligned along the $z$-axis. From the Boltzmann equation, we have a higher population of spins in the lower $|\alpha\rangle$ state than in the $|\beta\rangle$ state. Which in turn results in a net polarization along the $z$-axis known as the bulk magnetization vector, $\mathrm{M}$.

\subsection{Classical Description}

Now that we have shown how the bulk magnetization arises, we can move away from the quantum mechanical description of NMR and instead use a classical description. The bulk magnetization as first introduced in section 2.5, will experience a torque when exposed to a magnetic field, this torque will change $\mathrm{M}$ by a rate given by

$$
\frac{d \mathbf{M}}{d t}=\gamma \mathbf{M} \times \mathbf{B}
$$

Solving this equation for $\mathbf{B}=\mathbf{B}_{\mathbf{0}}$ gives us equation 2.1.

This bulk magnetization is roughly four orders of magnitude smaller than the external magnetic field, making detection in the $\mathbf{B}_{\mathbf{0}}$ direction impractical. To detect the bulk magnetization, a transverse magnetic field, $\mathbf{B}_{\mathbf{1}}$ is applied which 

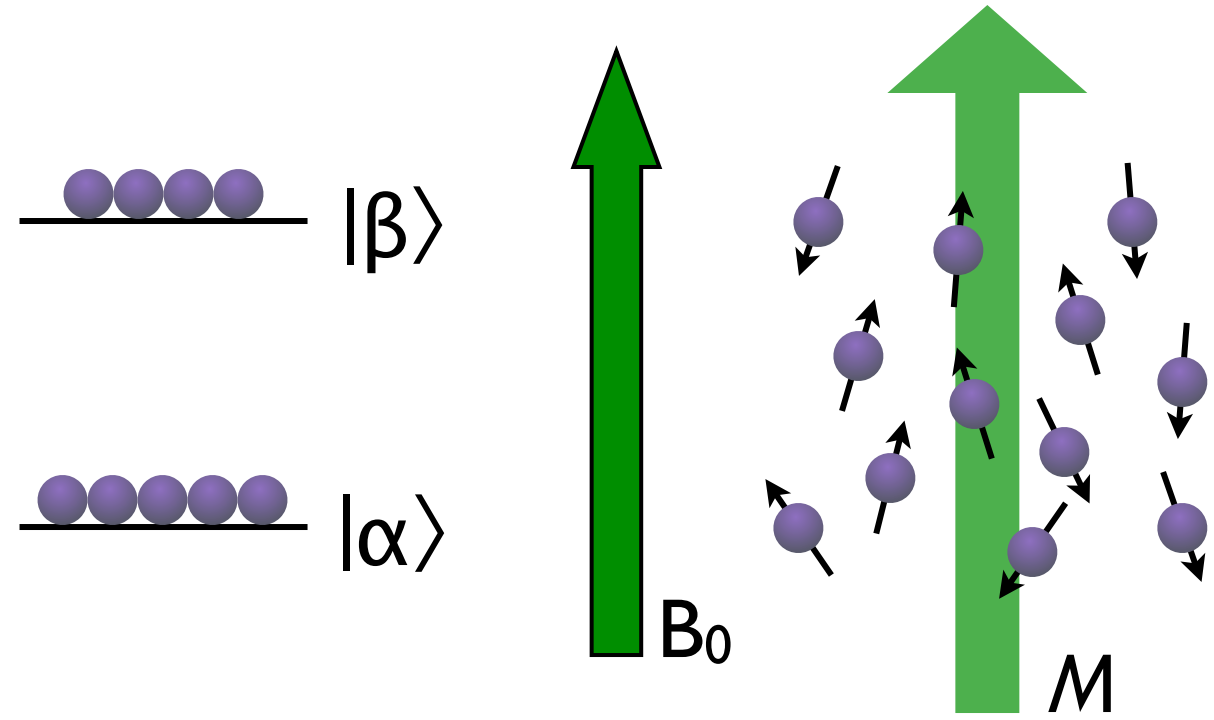

Figure 2.3: A spin ensemble in thermal equilibrium within a magnetic field. A net polarization forms parallel to the direction of $B_{0}$. This net polarization is known as the bulk magnetization vector, $M$.

oscillates at the Larmor frequency, $\omega_{0}$ of the bulk magnetization. This transverse magnetic field is given by the equation

$$
\mathbf{B}_{\mathbf{1}}(t)=2 \mathbf{B}_{\mathbf{1}} \cos \omega_{0} t \hat{i}
$$

which is comprised of two counter rotating components

$$
\begin{aligned}
\mathbf{B}_{\mathbf{1}}(t) & =\mathbf{B}_{\mathbf{1}} \cos \omega_{0} t \hat{i}-\mathbf{B}_{\mathbf{1}} \sin \omega_{0} t \hat{j} \\
\mathbf{B}_{\mathbf{1}}(t) & =\mathbf{B}_{\mathbf{1}} \cos \omega_{0} t \hat{i}+\mathbf{B}_{\mathbf{1}} \sin \omega_{0} t \hat{j} .
\end{aligned}
$$

One of these two components in equation 2.19 rotates in synchrony with the spins and is able to interact with and reorient them. The other, counter rotating component, rotates opposite to the spins and its effect averages to zero.

We can find the evolution of $\mathrm{M}$ using equation 2.17 and the initial condition $\mathbf{M}(0)=M_{0} \hat{k}$ which gives 


$$
\begin{aligned}
& M_{x}=M_{0} \sin \omega_{1} t \sin \omega_{0} t \\
& M_{y}=M_{0} \sin \omega_{1} t \cos \omega_{0} t \\
& M_{z}=M_{0} \cos \omega_{1} t
\end{aligned}
$$

where $\omega_{1}=\gamma B_{1}$. Equation 2.20 tells us that when we apply a transverse magnetic field $B_{1}$ oscillating at $\omega_{0}$, the bulk magnetization vector will precess about the longitudinal field $B_{0}$ at a frequency $\omega_{0}$ and simultaneously precess about the transverse field $B_{1}$ at a frequency $\omega_{1}$. This transverse magnetic field is known as a radiofrequency, or r.f. pulse. The duration of the r.f. pulse defines the flip angle of the magnetization vector. This flip angle, $\beta$, is given by the equation

$$
\beta=\omega_{1} t_{p}
$$

where $t_{p}$ is the pulse duration. The longer the pulse duration, the larger the flip angle. If we change our frame of reference from the laboratory frame to a frame which rotates around $B_{0}$ at the same frequency as $B_{1}$, in other words, at frequency $\omega_{1}$, the application of an r.f. pules simply rotates the magnetization vector about the $B_{1}$ vector by angle $\beta$ as seen in figure 2.4.

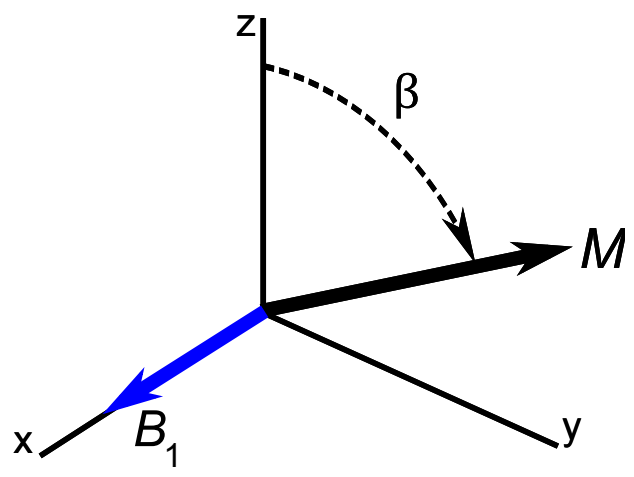

Figure 2.4: The magnetization vector in the rotating frame following an r.f. pulse. The magnetization vector will rotate about $B_{1}$ by an angle $\beta$. 


\subsection{Signal Detection}

It is shown by equation 2.20 that the application of an r.f. pulse will cause the bulk magnetization vector to precess around the longitudinal field $B_{0}$. This can also be expressed as an excitation of nuclear spins where they transition from the ground state energy level to a higher energy level.

If we have a solenoidal or saddle shaped r.f. coil whose winding axis is perpendicular to the $B_{0}$ field, we can detect the precessing transverse magnetization which will generate an electric current in the coil. This oscillating current is known as the NMR signal or free induction decay (FID). The FID will be discussed further in section 3.2.1.

\subsection{Relaxation and the Bloch Equations}

As discussed in section 2.5, the sample's thermal equilibrium state has a magnetization vector aligned with the longitudinal magnetic field, $B_{0}$, along the $z$-axis. An r.f. pulse will cause this magnetization vector to leave thermal equilibrium and precess in the transverse plane. As the magnetization vector is in the transverse plane it is exchanging energy with its surroundings which brings it back to thermal equilibrium. This process is known as longitudinal or spin-lattice relaxation, where the spin's surroundings is known as the "lattice". This relaxation has the form

$$
\frac{d M_{z}}{d t}=\frac{-\left(M_{z}-M_{0}\right)}{T_{1}}
$$

where $T_{1}$ is known as the spin-lattice or longitudinal relaxation time.

The nuclear spins in the sample also return to thermal equilibrium with themselves. This is known as spin-spin, or transverse relaxation which corresponds to the phase coherence between the spins. The transverse relaxation has the form

$$
\frac{d M_{x, y}}{d t}=\frac{-M_{x, y}}{T_{2}}
$$

where $T_{2}$ is known as the spin-spin or transverse relaxation time. 
Combining equations $2.17,2.22$, and 2.23 results in a set of equations in the rotating frame known as the Bloch equations

$$
\begin{aligned}
& \frac{d M_{x}}{d t}=\gamma\left(M_{y} B_{0}+M_{z} B_{1} \sin \omega t\right)-\frac{M_{x}}{T_{2}} \\
& \frac{d M_{y}}{d t}=\gamma\left(M_{z} B_{1} \cos \omega t-M_{x} B_{0}\right)-\frac{M_{y}}{T_{2}} \\
& \frac{d M_{z}}{d t}=\gamma\left(-M_{x} B_{1} \sin \omega t-M_{y} B_{1} \cos \omega t\right)-\frac{\left(M_{z}-M_{0}\right)}{T_{1}} .
\end{aligned}
$$

If $M$ is restored to thermal equilibrium along the $z$-axis there will be no magnetization present in the transverse plane and thus $T_{2}$ may be equal to or less than $T_{1}$, but can never be greater than $T_{1}$. However, it is important to note that the spins can also dephase due to inhomogeneities in $B_{0}$. This gives rise to an effective $T_{2}$, referred to as $T_{2}^{*}$ and is related to $T_{2}$ through the relationship

$$
\frac{1}{T_{2}^{*}}=\frac{1}{T_{2}}+\gamma \Delta B_{0}
$$

where $\Delta B_{0}$ is the spread in $B_{0}$. The effect of $T_{2}^{*}$ on the NMR signal will be discussed further in section 3.2.1.

\subsection{Mathematical Tools}

\subsubsection{Fourier Transform}

The NMR signal in the time domain may have features that are not easily recognizable by the human eye, such as the existence of multiple frequencies. There exists a mathematical tool known as the Fourier transform (FT) by which we can convert the NMR signal from the time domain to the frequency domain. Mathematically the FT of the function $f(t)$ is given by

$$
F(\omega)=\frac{1}{\sqrt{2 \pi}} \int_{-\infty}^{\infty} f(t) \exp ^{i \omega t} d t
$$

with the inverse FT giving $f(t)$ as

$$
f(t)=\frac{1}{\sqrt{2 \pi}} \int_{-\infty}^{\infty} F(\omega) \exp ^{-i \omega t} d \omega
$$


The FT of a complex signal will result in a spectrum of both real and imaginary parts, as shown in figure $2.5 \mathrm{~b}$. The real signal will take the form of a Lorentzian, shown by the blue solid line, and the imaginary signal will take the form of an dispersion spectrum.
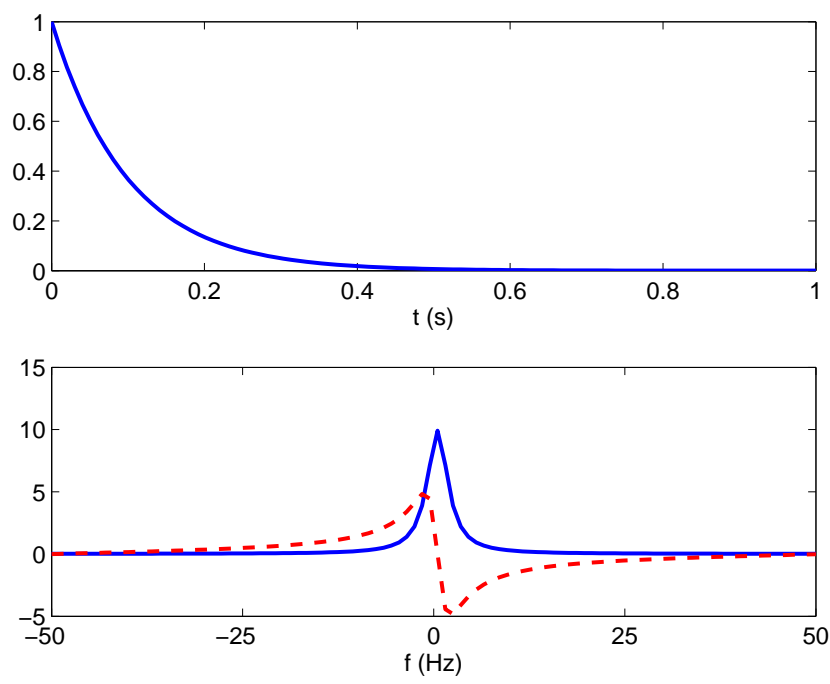

Figure 2.5: a). A simple example NMR signal. b). The Fourier transform of the signal shown in a). The real signal has a Lorentzian lineshape and is shown by the blue solid line. The imaginary signal is shown by the red dashed line and has the shape of an dispersion spectrum.

\subsubsection{Inverse Laplace Transform}

In cases where we seek to obtain characteristic decay times from NMR data rather than frequencies, we use the inverse Laplace transform, which is given by [9]

$$
f(s)=\frac{1}{2 \pi i} \int_{-i \infty}^{+i \infty} \exp (s t) F(t) d t
$$

where the Laplace transform is given by 


$$
F(t)=\int_{0}^{\infty} \exp (-s t) f(s) d s
$$

The inverse Laplace transform (equation 2.29) is part of a class of integrals known as "Fredholm integrals of the first kind" [10] which have the form

$$
F(t)=\int_{a}^{b} K(t, s) f(s) d s
$$

where $K(t, s)$ is the "kernel" of the function. What we wish to do is find the function $f(s)$ given our experimental data $F(t)$ and our kernel.

Multiexponential NMR data typically is in the form of a Fredholm integral of the first kind $[11,12]$

$$
M_{i}(t)=\int_{a}^{b}\left(f\left(T_{2}\right) \exp \left(-t_{i} / T_{2}\right)+\epsilon_{i}(t)\right) d T_{2}, \quad i=1,2, \ldots, N
$$

where $f\left(T_{2}\right)$ is the unknown amplitude of the spectral component at a relaxation time $T_{2}$. The limits $a$ and $b$ are chosen such that the logarithm of the expected values of the relaxation are contained within these limits. $K(t, s)$ is given by $\exp \left(-t_{i} / T_{2}\right)$, which can be changed depending on the data being analyzed. For example, for diffusion data, we use the kernel $\exp \left(-\gamma^{2} D \delta^{2}(\Delta-\delta / 3) \mathbf{g}^{2}\right.$ which will be explained in section 3.2.6. Finally, $\epsilon$ is the experimental noise of the measurement.

What makes the inverse Laplace transform different from the Fourier transform is that an increase in $\exp (s t)$ can cause divergence in the solution $f(s)$. This divergence is exacerbated by noise in $F(t)$ and by finite precision errors. Another way of looking at it, is if we add the function $\sin (\omega s)$ to $f(s)$, the RiemannLebesque lemma $[13,14]$ states that for any kernel $K(t, s)$,

$$
\lim _{\omega \rightarrow \infty} \int_{a}^{b} K(t, s) \sin (\omega s) d s=0 .
$$

This means that even for small errors $\epsilon$ in $F(t)$ and an arbitrarily large amplitude $A$, there still exists an $\omega$ such that $f(s)=A \sin (\omega s)$ is a solution to equation 2.30 within the error $\epsilon$. This means we have an infinite solution set, which means we have no unique solution, thus making this an ill-posed problem. 
In 1982, Provencher [15] developed a method to solve the inverse Laplace transform using three main strategies: 1). Absolute prior knowledge of the system, for example we know that the function $f(s)$ is non-negative. 2). Statistical prior knowledge of the system forces an optimal solution to be found in which the mean-squared error is minimized. 3). The principle of parsimony says that out of the solutions remaining after the first two strategies have been applied, we must select the simplest one. In other words, we must select the solution that has the least amount of unknown information to minimize the possibility of artifacts. Provencher uses these three strategies in his ILT program known as CONTIN [16].

To satisfy the principle of parsimony, Provencher makes use of Tikhonov regularization [17]. Then we discretize the integral and treat the solution $f(s)$ as a vector $\mathbf{f}$, the signal as a vector $\mathbf{F}$, and the kernel as a matrix $\mathbf{K}$, equation 2.30 becomes

$$
\mathbf{F}=\mathbf{K f}
$$

Using a least squares fit for $\mathbf{f}$, the residual will be $\|\mathbf{K f}-\mathbf{F}\|^{2}$. Tikhonov regularization adds a term $\Gamma \mathbf{f}$ to the residual, so we want to minimize the expression

$$
\chi^{2}(\alpha)=\|\mathbf{K f}-\mathbf{F}\|^{2}+\alpha^{2}\|\mathbf{\Gamma} \mathbf{f}\|^{2}
$$

where $\alpha^{2}$ is called the regularization parameter. $\Gamma$ is chosen such that the principle of parsimony is retained, i.e. we wish to have the minimum number of peaks in our inversion, for example, we can set our smoothness parameter to incorporate the second derivative of the solution

$$
\|\mathbf{\Gamma} \mathbf{f}\|^{2}=\int_{s_{\min }}^{s_{\max }}\left[f^{\prime \prime}(s)\right]^{2} d s
$$

where $s_{\min }$ and $s_{\max }$ are the bounds of our solution vector.

From equation 2.33 equation 2.34 becomes

$$
\chi^{2}(\alpha)=\sum_{i}^{N} \sum_{j}^{M}\left(F_{i}-K\left(t_{i}, s_{j}\right) f_{j}\right)^{2}+\alpha^{2} \sum_{j}^{M}\left(2 f_{j}-f_{j+1}-f_{j-1}\right)^{2} .
$$

where the last term is the numerical form of the second derivative of $f(s)$. 
We know our solution must be non-negative, so using that information, we seek to minimize $\chi^{2}$ for a range of $\alpha$ such that the value of $\chi^{2}$ is just approaching its "heel" as shown in figure 2.6a at around $\alpha^{-1}=10^{5}$. A value of $\alpha$ chosen at a greater value of $\chi^{2}$ will result in an overly smoothed inversion as seen in figure 2.6b, and an $\alpha$ after the heel can result in an under smoothed inversion which is unstable as shown in figure 2.6d. An extremely narrow $T_{2}$ distribution is indicative of an undersmoothed inversion and can result in pearling effects, which break the distribution up into multiple shark peaks. Figure 2.6c shows the inversion generated by choosing an $\alpha$ near the heel of figure 2.6a.
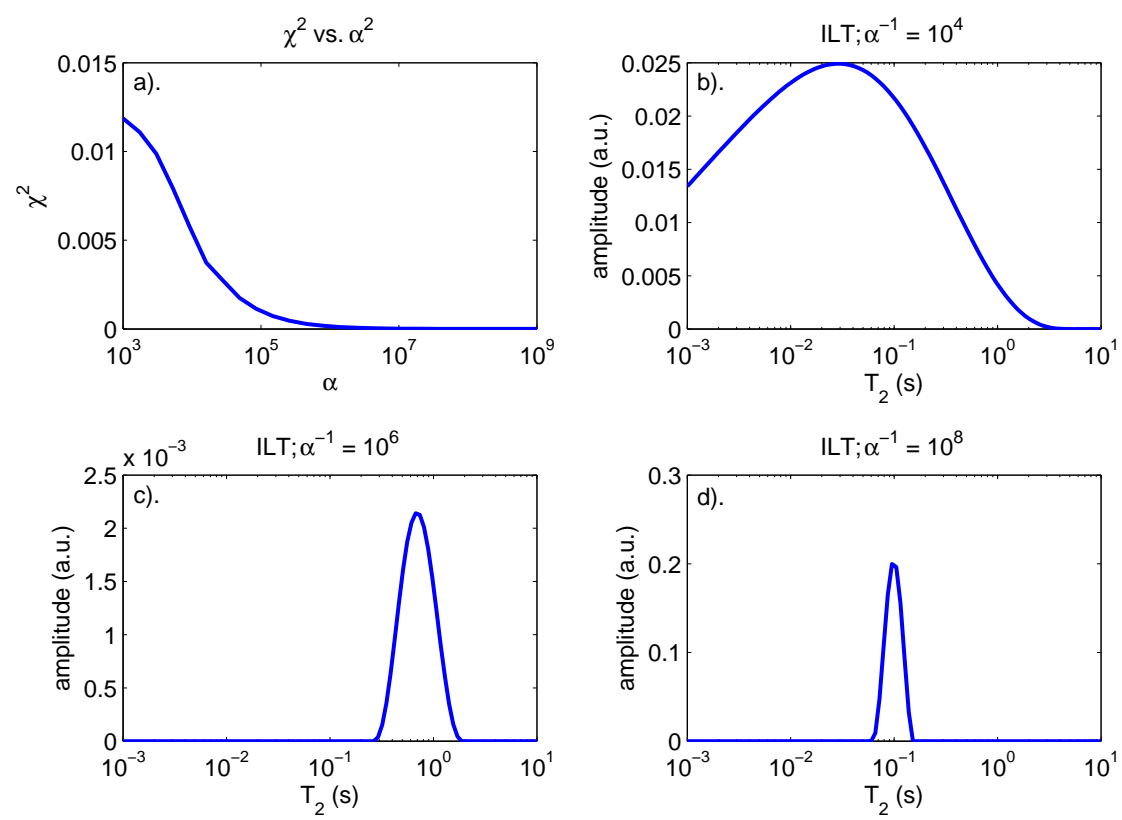

Figure 2.6: a). The regularization parameter, $\alpha^{-1}$ is chosen by selecting the value of $\alpha^{-1}$ which just minimizes $\chi^{2}$. b). An ILT for a single exponential with $\alpha^{-1}=10^{4}$. c). Same as b). but with $\alpha^{-1}=10^{6}$. d). As for b). with $\alpha^{-1}=10^{8}$. 


\section{Chapter 3}

\section{Pulse Sequences}

\subsection{Introduction}

Pulse sequences are the series of radio frequency pulses, delay times, and occasionally magnetic field gradient pulses which allow us to measure various characteristics of the spin system. In this section we introduce some of the fundamental pulse sequences of NMR. These pulse sequences range from the most basic FID comprised of a single r.f. pulse and an acquisition time to a Cotts sequence which uses multiple r.f. pulses, gradient pulses, and delay times. The knowledge of these fundamental pulse sequences will allow us to develop our own pulse sequences later on.

\subsection{One Dimensional NMR}

All one dimensional pulse sequences are made up of two parts: preparation and acquisition. In the preparation phase we bring the spin system to a desired state and then detect the resultant signal during the acquisition phase.

\subsubsection{Free Induction Decay}

The most basic NMR pulse sequence is comprised of a single $90^{\circ}$ pulse which rotates the magnetization vector into the transverse plane (figure 3.1). The mag- 
netization vector after this r.f. pulse is given by the equation

$$
M(t)=\left[M_{0} \cos \omega_{0} t \hat{i}+M_{0} \sin \omega_{0} t \hat{j}\right] \exp \left(-t / T_{2}\right)
$$

which shows that the magnetization after the pulse oscillates at the Larmor

$$
\text { r.f. }
$$
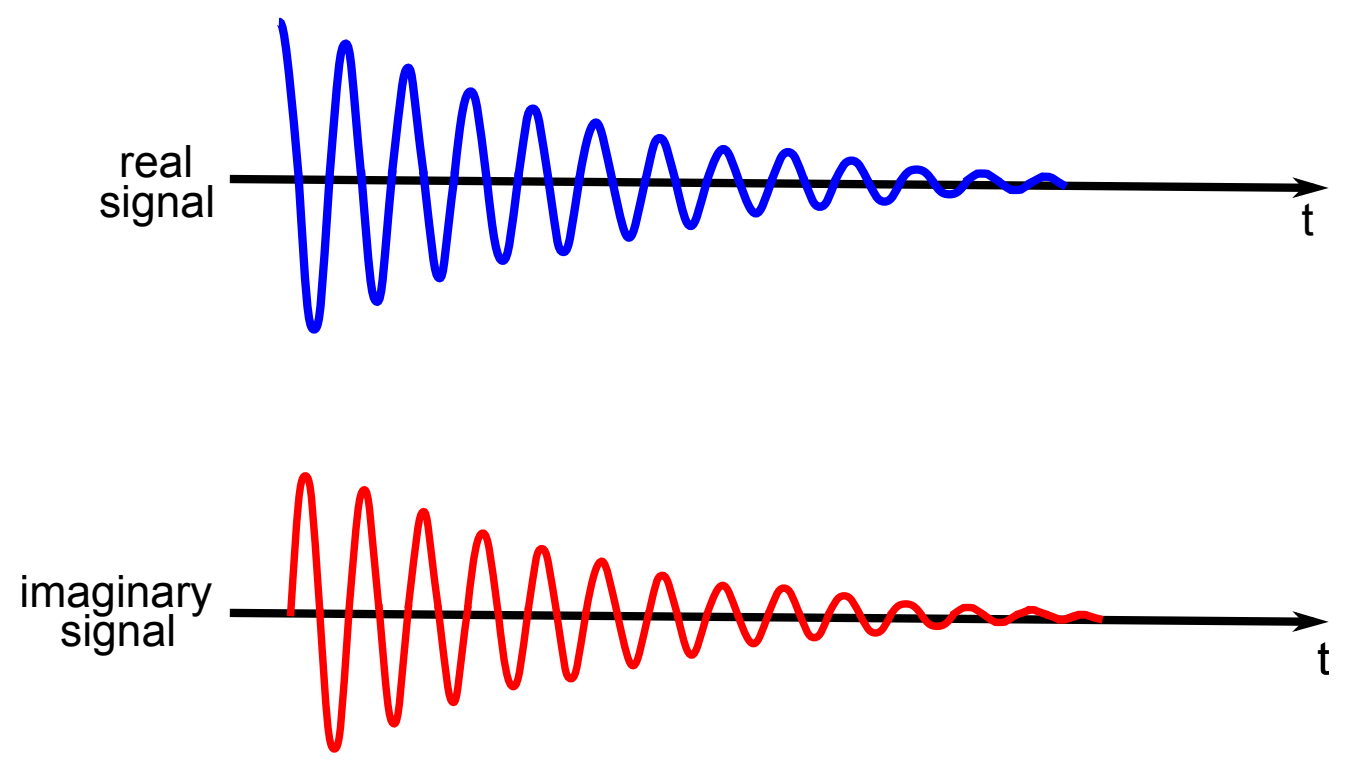

Figure 3.1: The FID pulse sequence. This produces a real signal and an imaginary signal as shown by the blue and red lines respectively.

frequency, $\omega_{0}$, and decays at a rate $T_{2}$. The $\mathrm{x}$ and $\mathrm{y}$ components of the magnetization generate two signals in the receiver called $M_{x}$ and $M_{y}$, which are known as the real and imaginary part of the signal respectively. To present this data in the frequency domain rather than the time domain, we can apply a Fourier transform as introduced in section 2.9.1. In the frequency domain, the real data will appear as a Lorentzian peakshape centered at the Larmor frequency, with 
a full width half maximum (FWHM) of $1 / \pi T_{2}$. This peakshape is also known as the absorption spectrum which is shown in figure $2.5 \mathrm{~b}$. The imaginary signal in the frequency domain will produce a dispersion Lorentzian also shown in figure $2.5 \mathrm{~b}$, which is slightly broader than the absorption spectrum.

It is important to note that an NMR sample in an external magnetic field, $B_{0}$, will experience magnetic field inhomogeneities, and the FID signal will decay faster than it would just from the $T_{2}$ relaxation alone. The resultant time constant from these field inhomogeneities is known as $T_{2}^{*}$ to differentiate it from the relaxation due to coherence loss, $T_{2}$. This line broadening caused by $T_{2}^{*}$ is known as inhomogeneous broadening, and this coherence loss due to the inhomogeneous magnetic field is able to be "corrected" by refocusing, whereas homogeneous broadening caused by coherence loss due to relaxation is irreversible as it is caused by the random motion of the spins.

\subsubsection{Inversion Recovery}

As defined in section 2.8, longitudinal relaxation, $T_{1}$ is caused by the exchange of energy of a precessing nuclear spin in an external magnetic field with its surroundings. Solving equation 2.22, the magnetization's return to equilibrium is given by

$$
M_{z}(t)=M_{z}(0) \exp \left(-t / T_{1}\right)+M_{0}\left(1-\exp \left(-t / T_{1}\right)\right) .
$$

A common way to measure $T_{1}$ is via a pulse sequence known as inversion recovery [18] shown in figure 3.2a.

This pulse sequences begins with a single $180^{\circ}$ pulse which brings the magnetization into the $-z$-axis. After a time $\tau$, a $90^{\circ}$ pulse brings the magnetization into the transverse plane where it is detected. The signal amplitude during this pulse sequence is given by the equation

$$
M(t)=M_{0}\left[1-2 \exp \left(-\tau / T_{1}\right)\right] .
$$

This tells us that at short $\tau$, the signal amplitude is negative, and as $\tau$ increases, this amplitude passes through zero and becomes positive, eventually reaching a plateau at $M_{0}$ (see figure 3.2b). 
a.

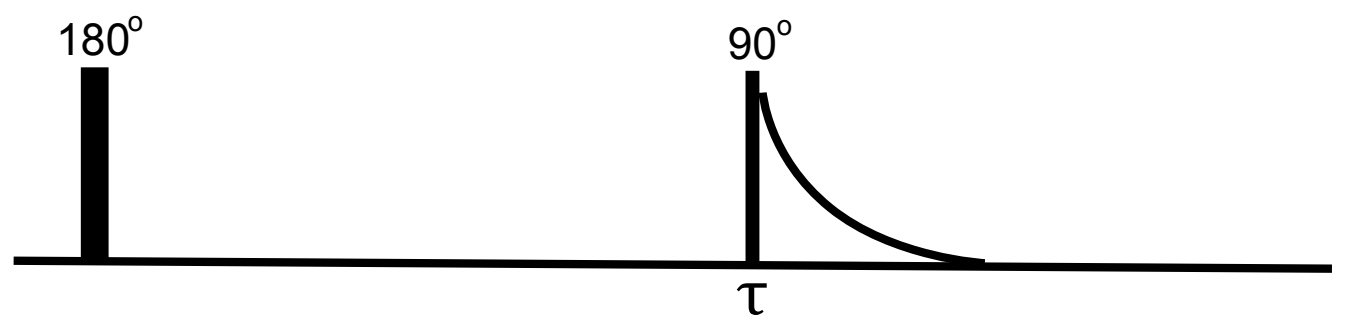

b.

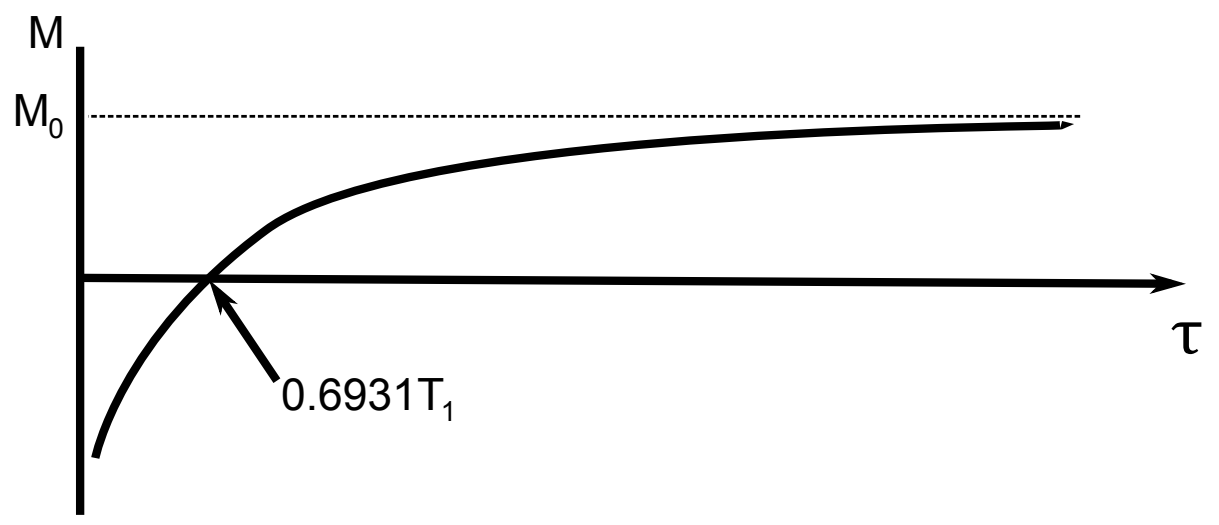

Figure 3.2: a). The inversion recovery sequence used to measure $T_{1}$ relaxation. b). The signal amplitude increases with $\tau$ and eventually reaches a plateau at $M_{0}$. The point where the signal becomes positive is equal to $0.6931 T_{1}$.

\subsubsection{Spin Echo}

As mentioned in section 3.2.1 we stated that the FWHM of the absorption spectrum is given by $1 / \pi T_{2}$. However, due to inhomogeneous broadening caused by macroscopic field inhomogeneities in the sample, we cannot use the FWHM of an FID spectrum to measure $T_{2}$. A method to separate the inhomogeneous broadening with the homogeneous relaxation based broadening was developed by Hahn in 1950 [19].

As discussed in section 2.8, the spins in the sample will eventually lose phase coherence due to interactions with their neighboring spins and the inhomogeneous magnetic field. Hahn discovered that this decoherence due to the magnetic field inhomogeneity is reversible upon application of a second r.f. pulse.

Referring to figure 3.3, if we apply a $180^{\circ}$ pulse at a time $\tau$ after the initial $90^{\circ}$ 
pulse, a refocusing of the phase coherence will appear at a time $2 \tau$ after the $90^{\circ}$ pulse.

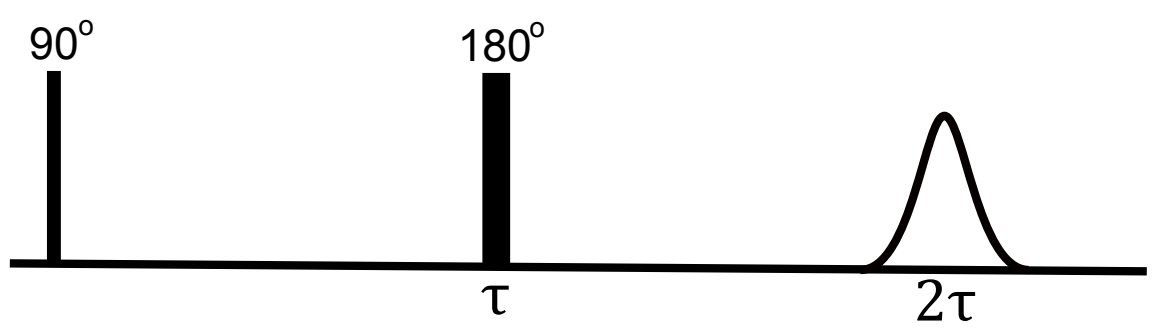

Figure 3.3: The spin echo pulse sequence. A spin echo will appear at a time $2 \tau$ after the $180^{\circ}$ pulse.

\subsubsection{CPMG}

As stated above, the spin echo gives us a method to separate broadening caused by $T_{2}$ from broadening caused by macroscopic field inhomogeneities, however, after the echo peak we will lose the phase coherence of the spins. Therefore, to measure $T_{2}$ using the pulse sequence shown in figure 3.3, we must repeat the measurement, increasing $\tau$ each time. A more efficient way of measuring $T_{2}$ was developed in 1954 by Carr and Purcell [20], in which they added a train of $180^{\circ}$ pulses after the initial $90^{\circ}$ pulse. If the $180^{\circ}$ pulses in the train are not exact, small angle errors will occur which will cause an error in the result. To compensate for this, Meiboom and Gill in 1958 [21] add a $90^{\circ}$ phase shift to the initial $90^{\circ}$ pulse. This is known as a Carr-Purcell-Meiboom-Gill, or CPMG pulse sequence and is shown in figure 3.4. The envelope of the spin echoes produced by the CPMG will decay at a rate of $T_{2}$.

\subsubsection{Stimulated Echo}

In materials where $T_{2}$ is much shorter than $T_{1}$, we can "store" the coherence over a time interval, $T$, for later recall. This method could be used for an application such as tracking how far spins have moved in a sample. The stimulated echo pulse sequence is shown in figure 3.5. 


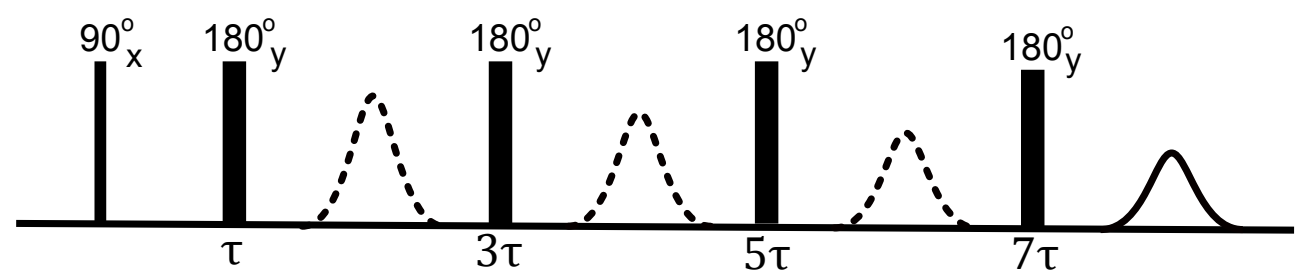

Figure 3.4: The Carr-Purcell-Meiboom-Gill (CPMG) pulse sequence used for measuring $T_{2}$. The envelope produced by the spin echoes will decay at a rate of $T_{2}$. Note, the phase difference between the $90^{\circ}$ and $180^{\circ}$ pulses must be $\pi / 2$ as this will cancel out any accumulating phase errors if the $180^{\circ}$ pulse is not exact.

A $90^{\circ}$ r.f. pulse brings the magnetization into the transverse plane where it experiences dephasing. A second $90^{\circ} \mathrm{rf}$ pulse at time $\tau$ brings it into longitudinal polarization along the $z$-axis. After a time $T$, a third $90^{\circ}$ pulse brings it back to the transverse plane where a stimulated echo appears at a time $T+\tau$.

Essentially the big difference between a spin echo and a stimulated echo is that for a stimulated echo a storage pulse is used to store the transverse magnetization along the $z$-axis. This is typically used in materials where the motion is very slow and $T_{2}$ is much shorter than $T_{1}$. This means that the spin polarization lasts longer than the spin coherence. It's this long lasting spin polarization that allows us to take advantage and store it along the $z$-axis.

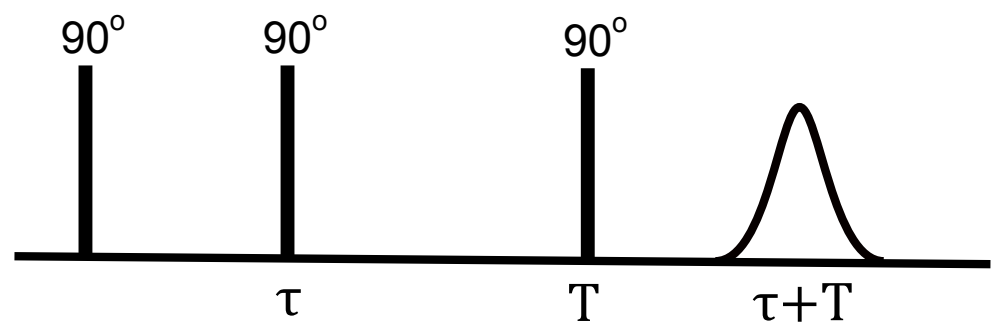

Figure 3.5: The stimulated echo pulse sequence. The splitting of the $180^{\circ}$ pulse into two $90^{\circ}$ pulses allows us to store the magnetization along the z-axis between time $\tau$ and $T$ and eliminate effects of $T_{2}$ relaxation. The stimulated echo will appear at a time $\tau+T$ after the initial $90^{\circ}$ pulse. 


\subsubsection{PGSE}

From equation 2.1, we know that the frequency a spin precesses at is dependent upon the external magnetic field. We can apply a linearly varying magnetic field which is much smaller than the polarizing field, $B_{0}$. Applying this known magnetic field gradient to the sample does two things: 1) because the applied gradient is much smaller than the longitudinal field, $B_{0}$, only those components of the gradient parallel to $B_{0}$ will affect the Larmor frequency. 2). components of the gradient orthogonal to the $z$-axis will serve to slightly rotate the net field direction. As the slight rotation has a negligible effect, we can write the Larmor frequency under an applied magnetic field gradient as

$$
\omega(r)=\gamma B_{0}+\gamma \mathbf{g} \cdot \mathbf{r}
$$

where $\mathrm{g}$ is the grad of the applied magnetic field gradient parallel to $B_{0}$ and $\mathbf{r}$ is the nuclear spin position.

Since the applied gradient will impart a Larmor frequency on the spins as a function of position, we can use the gradient to encode the spatial distribution of spins. Gradients can be thought to add phase to spins. The use of gradients in information storage with spin echoes was first described by Anderson in 1955 [22], and further refined by McCall in 1963 [23], in which they suggest the use of gradients to measure the self-diffusion coefficient. In 1965, Stejskal and Tanner demonstrated the use of a pulsed gradient spin echo (PGSE) pulse sequence (figure 3.6) as a way of measuring the self-diffusion coefficient.

The first $90^{\circ}$ excitation pulse brings the spins into the transverse plane. The applied gradient will change the Larmor frequency of the spins of the system along the gradient. So spins at position $z_{1}$ have a Larmor frequency of $\omega_{1}$ and spins at position $z_{2}$ have a Larmor frequency $\omega_{2}$. These different frequencies will cause the spins to lose coherence, another way to look at this is that the spins gain a phase $\phi_{1}$. After the $180^{\circ}$ pulse is applied, a second gradient is applied which "reverses" the dephasing the first gradient caused. In other words, after the second gradient, if the spins have remained in the same position, they will lose the phase $\phi_{1}$. However, if a spin has changed position or diffused during time $\Delta$, that spin will still have a phase shift after the second gradient. This 


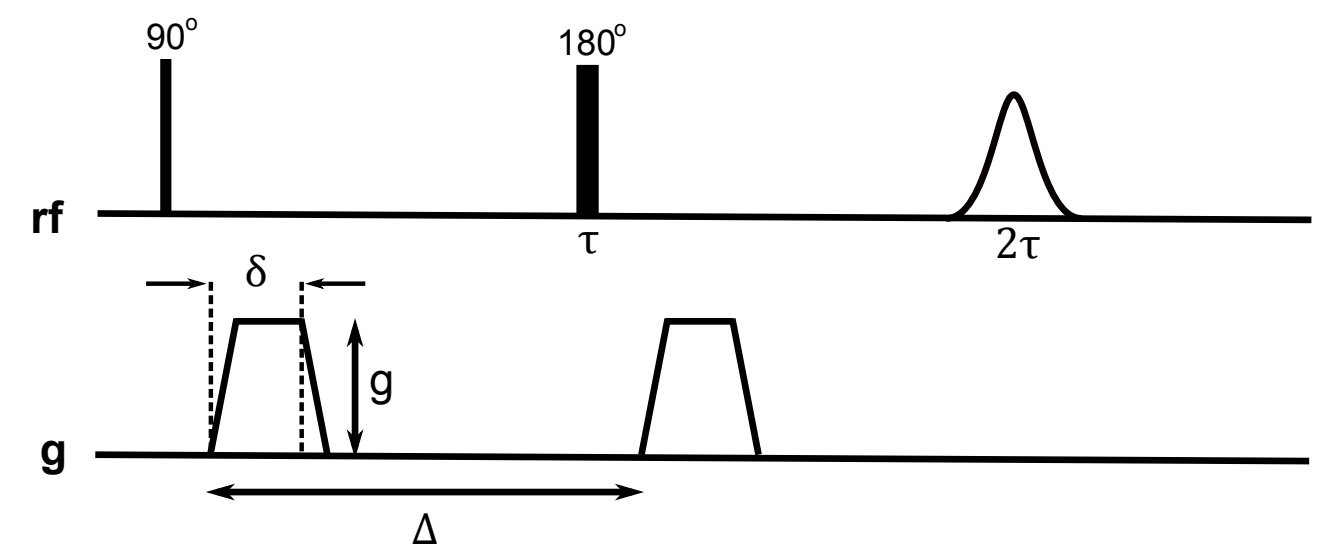

Figure 3.6: PGSE pulse sequence used to measure the self-diffusion coefficient.

phase will contribute to echo attenuation.

Given a volume of nuclear spins $d V$ at position $\mathbf{r}$, the local spin density will be $\rho(\mathbf{r})$, which means there are $\rho(\mathbf{r}) d V$ spins in the volume $d V$. We can write the NMR signal as

$$
d S(\mathbf{g}, t)=\rho(\mathbf{r}) d V \exp \left[i\left(\gamma B_{0}+\gamma \mathbf{g} \cdot \mathbf{r}\right) t\right]
$$

Since the reference frequency is $\gamma B_{0}$, the signal obtained oscillates at $\mathbf{g} \cdot \mathbf{r}$. This means that we can neglect the $B_{0}$ term in the above equation which gives us our integrated signal amplitude:

$$
S(t)=\iiint \rho(\mathbf{r}) \exp [i \gamma \mathbf{g} \cdot \mathbf{r} t] d \mathbf{r} .
$$

Formation of an echo in the presence of a field gradient depends on the assumption that the nuclear spins experience the same Larmor frequency during the dephasing and rephasing parts of the sequence. This assumption requires that the nuclei don't have any translational motion along the gradient direction. In liquids, translational motion is inevitable due to molecular self-diffusion. This movement will lead to random fluctuations in Larmor frequency and thus a distribution of residual phase shifts at the echo center.

Self-diffusion can be thought of as a "random walk" where motion is resolved in the direction of the field gradient. In a random walk, a molecule has an equal probability of moving to the left or right for a given step. Given an 
r.m.s displacement of $\xi$ for each step and a mean step time of $\tau_{s}$, the distance traveled after $n$ jumps at a time $t=n \tau_{s}$ is given by

$$
Z\left(n, \tau_{s}\right)=\sum_{i=1}^{n} \xi a_{i}
$$

where $a_{i}$ is a random number equal to \pm 1 . $Z$ is the $z$-axis displacement of the molecules.

The self-diffusion constant in one dimension is defined as

$$
D=\xi / 2 \tau_{s}
$$

and

$$
\overline{Z^{2}(t)}=2 D t
$$

We can use the attenuation of the spin echo from diffusive dephasing under the influence of a pulsed gradient to measure molecular self-diffusion by using a pulsed gradient spin echo (PGSE) technique (see Figure 3.6) first developed by Stejskal and Tanner in 1965 [24]. The Stejskal-Tanner equation for the echo attenuation is given by

$$
S(\mathbf{g}) / S(0)=E(g)=\exp \left[-\gamma^{2} \mathbf{g}^{2} \delta^{2} D(\Delta-\delta / 3)\right] .
$$

We can find the self-diffusion coefficient, $D$ by plotting $\ln (E(\mathbf{g}))$ as a function of $\gamma^{2} \mathbf{g}^{2} \delta^{2}(\Delta-\delta / 3)$ and fitting a straight line to the resulting trend. $D$ will be the slope of the fit line. Units are $\mathrm{m}^{2} \mathrm{~s}^{-1}$.

Torrey [25] recognized that diffusion would affect the magnetization and added a diffusion term to the Bloch equations (equation 2.24) to create the Bloch equations with diffusion, also known as the Bloch-Torrey equations

$$
\begin{aligned}
& \frac{d M_{x}}{d t}=\gamma\left(M_{y} B_{0}+M_{z} B_{1} \sin \omega t\right)-\frac{M_{x}}{T_{2}}+\nabla \cdot D \nabla M_{x} \\
& \frac{d M_{y}}{d t}=\gamma\left(M_{z} B_{1} \cos \omega t-M_{x} B_{0}\right)-\frac{M_{y}}{T_{2}}+\nabla \cdot D \nabla M_{y} \\
& \frac{d M_{z}}{d t}=\gamma\left(-M_{x} B_{1} \sin \omega t-M_{y} B_{1} \cos \omega t\right)-\frac{\left(M_{z}-M_{0}\right)}{T_{1}}+\nabla \cdot D \nabla M_{z} .
\end{aligned}
$$




\subsubsection{PGSTE}

Using PGSE for measurement of the self-diffusion coefficient over a long storage time, $\Delta$, can be limited by phase coherence loss due to $T_{2}$ relaxation. By splitting the $180^{\circ}$ pulse into two $90^{\circ}$ pulses, we can convert the spin echo PGSE to a stimulated echo which will allow us to eliminate the effects of $T_{2}$ relaxation by storing the magnetization along the $z$-axis. The spins will still be affected by $T_{1}$ relaxation, which is slower than transverse relaxation. This method was first proposed by Tanner in 1970 [26], and is advantageous in situations where a long $\Delta$ is needed, such as a sample which is highly viscous, which will result in a short $T_{2}$ and low self-diffusion coefficient, or simply when a long $\Delta$ time is of interest. The technique is known as a pulsed gradient stimulated echo (PGSTE) and is shown in figure 3.7 .

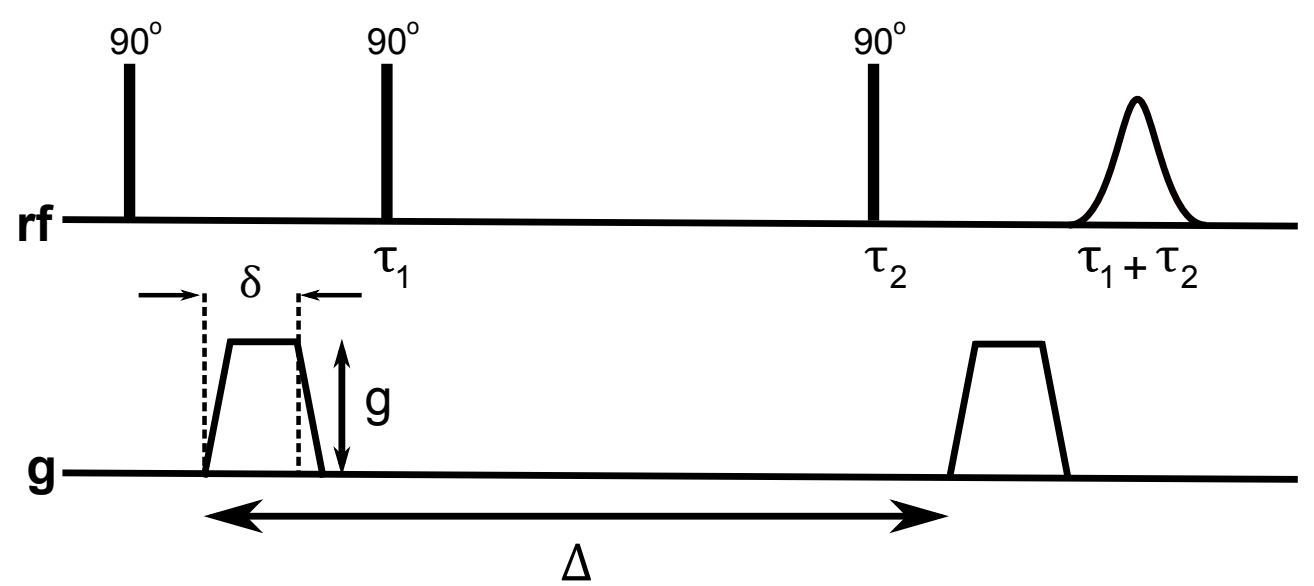

Figure 3.7: PGSTE pulse sequence, similar to figure 3.6, but uses a stimulated echo rather than a spin echo r.f. sequence.

\subsubsection{Bipolar Gradients}

In samples where there are large internal magnetic field inhomogeneities present the measurement of diffusion can become inaccurate due to a loss of signal from a decrease in $T_{2}$ relaxation, causing the $T_{2}$ time to shorten. In 1970, Packer et al [27] showed empirically that the addition of two gradient pulses in a CPMG sequence could be used to measure $D$ provided diffusion was slow. This method 
was expanded upon by Williams et al in 1978 [28] in which the gradients are placed in specific places in the CPMG train which allows one to measure $D$ independently of background gradients. In 1980, Karlicek and Lowe [29] developed the alternating PFG (APFG) technique which is comprised of $2180^{\circ}$ pulses between which are placed gradients of alternating signs. Cotts et al [30] expanded upon this idea of bipolar gradients and combines them with a stimulated echo sequence to produce the 13-interval sequence most commonly used to eliminate effects of internal magnetic gradients. This sequence is shown in figure 3.8.

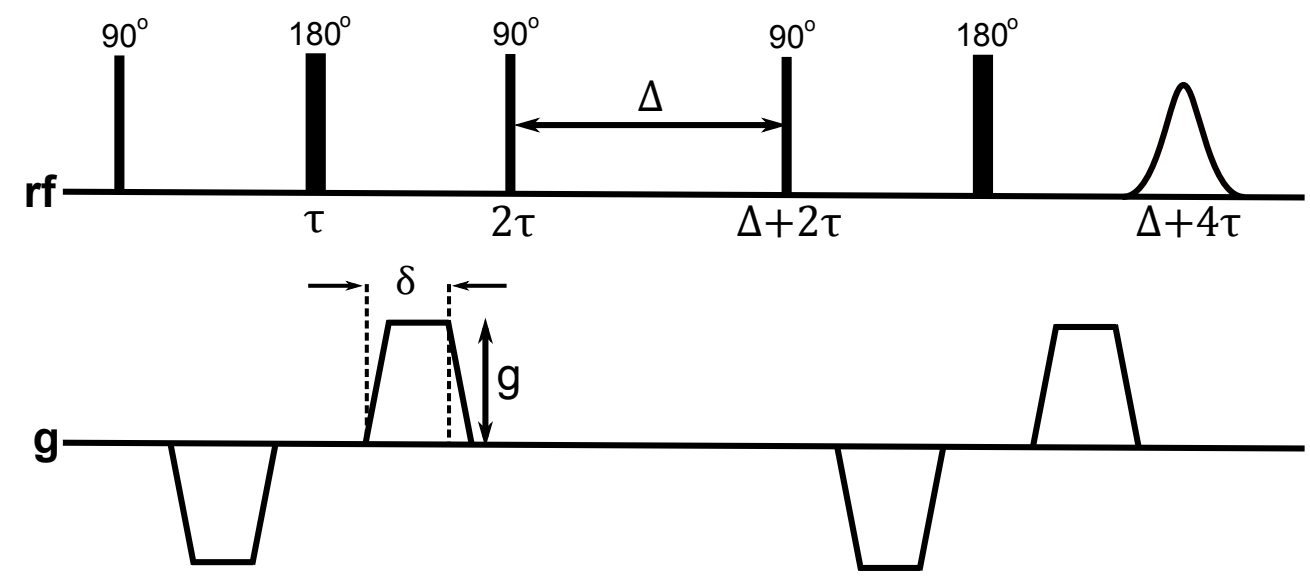

Figure 3.8: The 13-interval Cotts sequence, used to reduce the interaction of magnetic field inhomogeneities present in the sample and the pulsed field gradients used in the pulse sequence.

The spin echo parts around the $180^{\circ}$ r.f. pulse obviously refocuses the effects of inhomogeneous local fields, but in addition, cross terms between the applied gradient pulses and the inhomogeneous field are also eliminated. This latter effect depends upon the gradient pulses being symmetrically placed within the echo interval [30].

\subsubsection{Propagators and q-space}

By making the time, $\delta$, of the applied gradients in figure 3.6 very small with respect to $\Delta$, we can neglect diffusion of the spins over $\delta$ and we can get information about general translational motion, not just diffusion but also information about the velocity correlation function $\overline{v_{z}(0) v_{z}(t)}$ and the propagator, $P_{s}$ 
described later. In this thesis, we do not make much use of the velocity correlation function, so we will not expand upon it here.

The first pulsed gradient will give the spins a phase shift of $\gamma \delta \mathbf{g} \cdot \mathbf{r}$ for a spin located at position $\mathbf{r}$ at the time of the pulse. A change in position to $\mathbf{r}^{\prime}$ at the time of the second gradient pulse will impart a net phase shift of $\gamma \delta \mathbf{g} \cdot\left(\mathbf{r}^{\prime}-\mathbf{r}\right)$ causing an attenuation of the echo signal, $E(\mathbf{g}, \Delta)$, defined as the maximum amplitude at the center of the echo. The total signal is a superposition of phases, $\exp \left[i \gamma \delta \mathbf{g} \cdot\left(\mathbf{r}^{\prime}-\mathbf{r}\right)\right]$ each weighted by the probability of a spin to move from $\mathbf{r}$ to $\mathbf{r}^{\prime}, \rho(\mathbf{r}) P_{s}\left(\mathbf{r} \mid \mathbf{r}^{\prime}, t\right)$. Therefore we have

$$
E(\mathbf{g}, \Delta)=\int \rho(\mathbf{r}) \int P_{s}\left(\mathbf{r} \mid \mathbf{r}^{\prime}, \Delta\right) \exp \left[i \gamma \delta \mathbf{g} \cdot\left(\mathbf{r}^{\prime}-\mathbf{r}\right)\right] d \mathbf{r}^{\prime} d \mathbf{r}
$$

We define a reciprocal space $\mathbf{q}$

$$
\mathbf{q}=\gamma \delta \mathbf{g}
$$

and define $\mathbf{R}=\mathbf{r}^{\prime}-\mathbf{r}$, we can rewrite equation 3.12 as

$$
E(\mathbf{q}, \Delta)=\int \bar{P}_{s}(\mathbf{R}, \Delta) \exp (i \mathbf{q} \cdot \mathbf{R}) d \mathbf{R}
$$

From equation 3.14 we can see there is a Fourier relationship between $E(\mathbf{q}, \Delta)$ and $\bar{P}_{s}(\mathbf{R}, \Delta)$. By acquiring the signal in q-space we are able to get information about the propagator, $\bar{P}_{s}(\mathbf{R}, \Delta)$.

$\overline{P_{s}}(\mathbf{R}, \Delta)$ is known as the propagator, or the probability a molecule has diffused a distance $\mathbf{R}$ during time $\Delta . \overline{P_{s}}(\mathbf{R}, \Delta)$ can be written as [31]

$$
\overline{P_{s}}(\mathbf{R}, \Delta)=(4 \pi D \Delta)^{-3 / 2} \exp \left(-\mathbf{R}^{2} / 4 D \Delta\right) .
$$

Short times result in a narrow propagator, which will broaden with increasing $\Delta$.

\subsection{Two-Dimensional NMR}

Two-dimensional NMR was first proposed by Jeener [32] at the Ampere International Summer School II. Unfortunately this was never published in a scientific 
journal, however, Ernst's group review Jeener's proposal in 1976 while giving full credit to Jeener [33]. 2D NMR is also covered in detail by Ernst [34]. There are times in which we cannot fully characterize a system by a one dimensional spectrum alone. In these cases we can expand the measurement into two dimensions by adding a second domain. In general, a 2D NMR experiment can be split up into four parts: preparation, evolution, mixing, and detection.

During the preparation time, we prepare the spin system by exciting it into the transverse plane. This may involve something as simple as a single $\pi / 2$ pulse or something more complex such as polarization transfer or enhancement of polarization. We then allow the system to evolve during the evolution time, $t_{1}$ which comprises the first time domain in our 2D experiment. Following the evolution time we have the mixing time. The mixing time is used to amplify the information gained from the experiment. It can be just a time, $\tau_{m}$ or can include pulses. Finally, the detection time, $t_{2}$, is the time when the NMR signal is acquired.

The application of a 2D Fourier transformation to the time domain data $t_{1}$ and $t_{2}$, will produce a 2D spectra with frequencies $f_{1}$ and $f_{2}$ with peaks at various frequencies. Peaks along the diagonal indicate areas where the signal did not change frequency between times $t_{1}$ and $t_{2}$. An off-diagonal peak indicates a change in frequency between the evolution and detection times. The appearance of these off-diagonal peaks is dependent on the mixing time, without which there would be only on-diagonal peaks.

\subsubsection{Two-Dimensional Exchange Experiments}

Two-dimensional exchange spectroscopy (EXSY) allows us to be able to track the exchange of spins within a system [34,35]. By frequency tagging spins during the evolution time, $t_{1}$, allowing them to exchange during the mixing time $\tau_{m}$, and measuring the exchange during the acquisition time, $t_{2}$, we are able to track the migration of molecules in the sample.

Relaxation exchange spectroscopy (REXY) was first presented by Lee in 1993 [36] who developed a pulse sequence which detects exchange between two species in a heterogeneous system. Diagonal peaks are formed by spins that have not 
undergone exchange during the mixing time, in other words, those spins whose $T_{2}$ remains the same before and after the mixing time. Off-diagonal peaks are formed when spins undergo sufficient exchange to change the $T_{2}$ rate. They tested the pulse sequence on samples of urea and water and found that they do see diagonal and off-diagonal peaks due to proton exchange.

In 2005, McDonald et al [37] extended Lee's work to studying cement pastes. In a preliminary experiment, they looked at the exchange of water between gel and capillary pores in cement gels that are hydrated between 1 and 4 days. This work was built upon and expanded in 2006 by Monteilhet et al [38] in which they simulated $T_{2}-T_{2}$ exchange spectra for mixing times and exchange rates. They found that with a high exchange rate off-diagonal peaks do not appear and instead there is one diagonal peak at a location given by the average $T_{2}$. Increasing $\tau_{m}$ results in a decreasing intensity across all peaks due to $T_{1}$ relaxation. The simulations were also run with added levels of noise and a baseline error to see how this would affect the Laplace inversion. They found that adding noise will not affect the position of the peaks, but would affect the intensity of the offdiagonal peaks. Adding a baseline error plus noise would change the position of some of the peaks, particularly the peaks occurring at both short $T_{2}^{1}$ and short $T_{2}^{2}$ times. Experiments were run on 4 day old cement paste samples at different mixing times. By analyzing the locations and intensities of the off-diagonal peaks, Monteilhet estimated the dominant pore sizes in the cement paste and calculated the water diffusivity.

Washburn and Callaghan [39] in 2006 used REXSY to investigate pore-pore exchange in Castlegate sandstone. To ensure that the off-diagonal peaks appear due to pore to pore exchange they used quantitative analysis. They found that growth of off-diagonal peaks and decay of diagonal peaks match nicely with theory.

Most recently, Kuntz et al [40] proposed a simple exchange experiment to study the frequency exchange in a water-filled glass bead sample and a polystyrene sample. For their experiments they used two glass bead packs with a mean diameter of $31 \mu \mathrm{m}$ and standard deviations of $5.6 \mu \mathrm{m}$ and $9.3 \mu \mathrm{m}$. The polystyrene sample had a mean diameter of $9.1 \mu \mathrm{m}$ and a standard deviation of $1.2 \mu \mathrm{m}$. They found growth along the diagonal, however their spectra for the glass beads were 
slightly asymmetrical, most likely as a result of the high standard deviation of their samples. The resultant spectra of the water for the polystyrene spheres were highly asymmetrical, which Kuntz attributed to the heterogeneous pore space due to the rough surface of the polystyrene found by SEM of the spheres.

\subsubsection{Two-dimensional Correlation Experiments}

Two-dimensional correlation spectroscopy (COSY) gives us a way to investigate the structure of the spin system by utilizing coherence transfer [34]. The 2D spectra obtained from COSY can give information about the coherence transfer pathways of the sample. In chemistry and biology, COSY is often used to determine the structure of complex molecules such as proteins. Coherence transfer is the exchange of coherence from one transformation to the other. J-couplings cause precession of simple magnetization into higher order terms in the density matrix (e.g. $I_{x} \rightarrow I_{y} I_{z}$ ). A second r.f. pulse applied to the sample can be thought of as applying a rotation operator, $R$, to the density matrix $\rho$ of the sample. For example, the rotation operator for a $\beta$ rotation about the $x$-axis is given by

$$
R_{x}(\beta)=\exp \left(-i \beta I_{x}\right) .
$$

The application of an r.f. pulse to, for example, $I_{y} I_{z}$, results in the generation of new coherences. This transfer of coherence results in cross peaks in a COSY spectrum. The intensity of the peaks is dependent on the amount of coherence transfer.

In porous media, we can use COSY to give us information about the sample's structure. For example, we can use a diffusion diffusion correlation experiment (DDCOSY) [41] to give us information about samples that are macroscopically isotropic but microscopically anisotropic such as lamellar phase liquid crystals [42, 43, 44], hollow capsules [45], or plant tissues [46]. By applying successive gradients that are either parallel or perpendicular with one another, we obtain either diagonal or off-diagonal features. Applying parallel gradients will result in measuring a distribution of molecular diffusion according to the direction between the applied gradient and the local principal axes, $D_{\|}$, while applying orthogonal gradients result in a different sensitivity to the distribution. 
Diagonal peaks arise during an application of a collinear gradient because we have repeated the same experiment twice. Upon an application of orthogonal gradients, off-diagonal intensities grow due to the local anisotropies in the system, with some spins having their local $D_{\|}$aligned with the first gradient pulse pair but orthogonal to the second pair. Others will, by chance, have similar diffusion for both pairs, and so contribute diagonal peaks. The pattern obtained tells us about the nature of local anisotropy.

Diffusion relaxation correlation spectroscopy (DRCOSY) allows us to correlate $T_{2}$ with corresponding diffusion coefficients which can enable us to distinguish between two fluids such as oil and water [47] or can allow us to determine where water is located within a porous medium such as hollow capsules [45] or plant structures [46]. For example, the $T_{2}$ distributions of oil and water are often similar, and their corresponding diffusion distributions are different since water typically diffuses faster than oil. By correlating $D$ and $T_{2}$, we are able to separate the water or oil signal contribution in the $T_{2}$ distribution, thus allowing one to distinguish between the two fluids.

Relaxation relaxation correlation spectroscopy (RRCOSY) [48] correlates $T_{1}$ with $T_{2}$ which is another way to investigate the pore space in porous media. This method has been used to study enzymes [49], muscle tissue [50], water filled rocks [51], and cements [37,38]. By correlating $T_{1}$ with $T_{2}$, one can find the $T_{1} / T_{2}$ ratio, and probe the exchange of protons within the porous system. 


\section{Chapter 4}

\section{Porous Media}

\subsection{Introduction}

Nearly all materials can be classified as porous in some way. Some examples of porous media include wood, rocks, bones, and even foods such as cake. Many industries are interested in the characterization of porous materials to gain more information about them. For example, the oil industry is interested in the pore size distribution and the fraction of the pore space containing oil or water in an oil reservoir, medical researchers can use information about the pore size of bones to diagnose osteoporosis. In this chapter we introduce some basic properties of porous materials and how the pore space affects NMR measurements. Some helpful books and reviews on porous media include [52, 53, 54, 55].

\subsection{Properties of Porous Media}

Of course, the major characteristic of a porous medium is that it's porous, in other words, it's comprised of two phases, a solid phase and a fluid (or vacuum) phase, which are also known as the matrix and pore space, respectively. However, we can describe porous media in terms of its various properties, including porosity, permeability, formation factor, tortuosity, wettiblity, and the pore size distribution. In this section we give a brief review of what these properties are in terms of the study of porous media. 


\subsubsection{Porosity and Representative Elementary Volume}

Porosity, $\phi$, is simply defined as the ratio of empty space in the material to that of the bulk volume. This value can vary from near zero to near unity depending on the material being examined.

Porosity can be determined using a variety of methods such as the direct method, where the bulk volume of material is measured and then pulverized to destroy all pore space. The volume of pulverized material is then measured via fluid displacement. Another method of measurement is the mercury injection method where the porous sample is placed in a vessel containing a known volume of mercury under a known pressure. The bulk volume of the sample is found by the displaced volume of mercury. To find the pore volume, the pressure in the vessel is increased by a volumetric pump so that the mercury saturates the pore space of the sample. Finally, it can also be measured via NMR where the porosity is proportional to the signal intensity of the fluid in a saturated sample.

Before continuing on to describe other porous media characteristics, it is important to define a representative elementary volume (REV). This is defined to be smallest volume over which we can make a statistical analysis around a point $P$. It must also be smaller than the entire sample, otherwise we will not have a meaningful analysis of what happens at $P$. One way to define the REV is by using the porosity of the sample. A good description of this can be found in Bear's book [54].

First we take a sample volume $\Delta V_{i}$ centered at a point $P$ and calculate the porosity of this volume as $\left.\phi_{i}=\left(\Delta V_{v}\right)_{i}\right) / \Delta V_{i}$ where $\left(\Delta V_{v}\right)_{i}$ is the volume of pore space within $\Delta V_{i}$. We repeat this for a series of $\Delta V_{i}$ where $i=1,2,3, \ldots$ and each successive volume is smaller than the last, i.e. $\Delta V_{1}>\Delta V_{2}>\Delta V_{3}$ and so on.

Referring to figure 4.1, if we begin at large values of $\Delta V_{i}$, and slowly reduce the volume, the value of $\phi_{i}$ is fairly constant but may undergo slight changes if the observed domain is inhomogeneous. As we keep decreasing $\Delta V_{i}$ we reach a region where $\phi_{i}$ begins to exhibit large fluctuations due to the dimensions of $\Delta V_{i}$ reaching the size of a single pore. Finally, as $\Delta V_{i} \rightarrow 0, \phi_{i}$ will converge to one if $P$ is in the matrix, or zero if $P$ is in a pore space. 


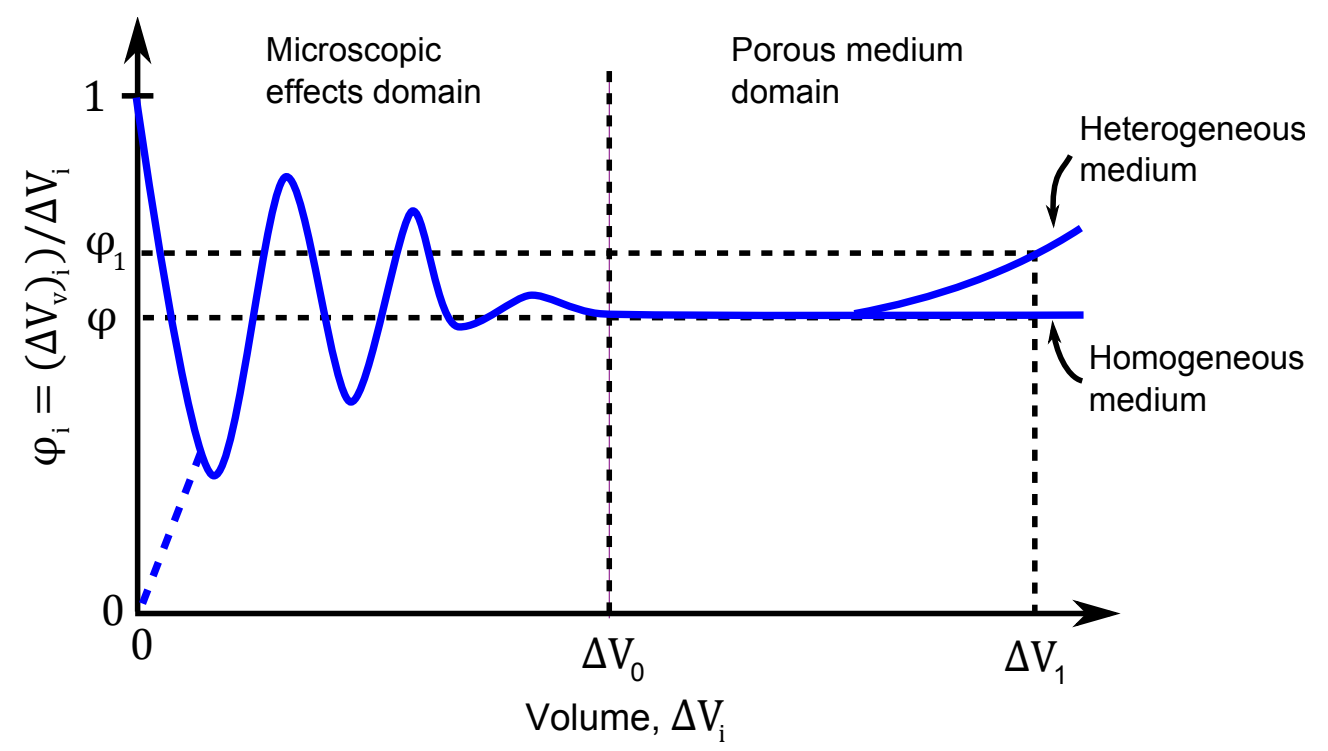

Figure 4.1: Finding the representative elementary volume. Adapted from [54].

If the pore space of a porous sample is distributed in an ordered or disordered fashion such that pore morphologies are indistinguishable, the sample is known as homogeneous and will approach $\phi$ with increasing $\Delta V_{i}$. However, if the pore space morphology varies in such a way that one is able to correlate pore size with position over the space of a few pores, the medium is then known as heterogeneous and will deviate slightly from $\phi$ depending on the pore geometry. Figure 4.2a shows an example of a homogeneous medium where the pore sizes do not depend on location. Contrasting this, in figure $4.2 b$, is an example of a heterogeneous medium where we have four clusters of smaller pores with a small pore separation that are dependent on pore location.

\subsubsection{Permeability}

The permeability, $K_{p}$ of a porous medium is a measure of how easily a fluid is able to flow through the medium. This value is dependent on the pore structure and the viscosity of the fluid and is given in units of darcy. A porous material has a permeability of 1 darcy if a pressure difference of $1 \mathrm{~atm}$ will cause a flow rate of $1 \mathrm{~cm}^{3} / \mathrm{s}$ of a fluid with a viscosity of $1 \mathrm{cP}$ through a cube of side $1 \mathrm{~cm} . K_{p}$ 
a).

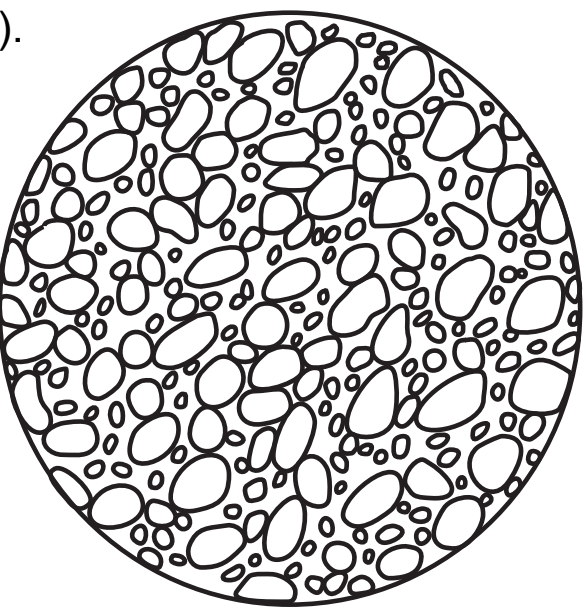

b).

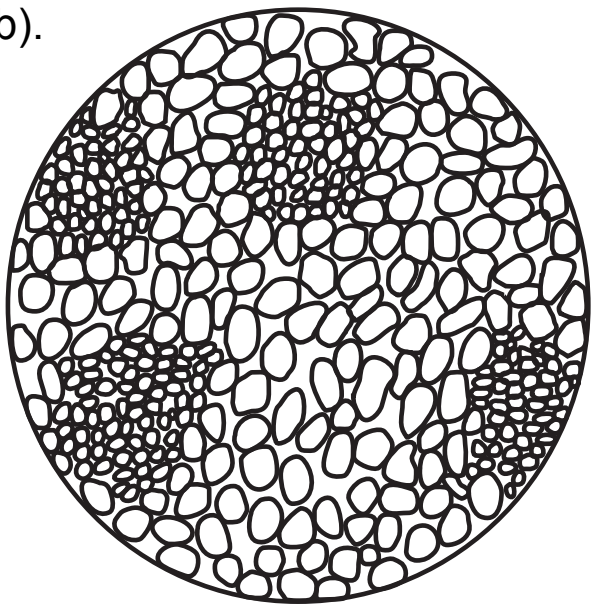

Figure 4.2: a). homogeneous pore structure. b). heterogeneous pore structure. Reproduced from [52].

is defined by Darcy's Law [4]:

$$
\left\langle v_{z}\right\rangle=-\frac{K_{p}}{\eta} \frac{d p}{d z}
$$

where $\eta$ is the fluid viscosity, and $\left\langle v_{z}\right\rangle$ is the average flow rate (velocity) along the pressure gradient, $d p / d z$. The average flow rate is calculated from the volumetric flow rate across an area $A$ as $\dot{Q}=\left\langle v_{z}\right\rangle A$.

While permeability can be measured using a steady state flow through a sample, these measurements can have a high experimental error. Due to its non-invasiveness, convenience and accuracy, NMR can be used as an alternative method of permeability measurement [56].

\subsubsection{Formation Factor and Tortuosity}

The formation factor, $F$, is an electrical property of fluid-filled porous media and is defined as [57]

$$
F=\frac{\rho_{o}}{\rho_{w}}
$$

where $\rho_{o}$ is the resistivity of a porous sample filled with an ionic fluid (typically a $\mathrm{NaCl}$ solution with a concentration greater than $10 \mathrm{~g} / \mathrm{L}$ ), and $\rho_{w}$ is the resistivity of a bulk volume of the same fluid. The formation factor is always greater than 
unity and is also related to the porosity of a sample by [58]

$$
F=\frac{D_{0}}{\phi D_{e f f}}
$$

where $D_{0}$ is the bulk diffusion coefficient, and $D_{\text {eff }}$ is the effective diffusion which is further discussed in section 4.4 and corresponds to the measured value after some particular diffusion time. For that reason, $D_{\text {eff }}$ is also referred to as $D(t)$. We can use the formation factor to find the tortuosity, $\alpha$ of the sample, which is a measure of the deviation from the macroscopic flow in a sample, in other words, a measure of flow direction changes. The tortuosity is given by

$$
\alpha=F \phi
$$

For the particular case of a bead pack, $\alpha \simeq 1 / \sqrt{\phi}$ [58], a result that will be used in chapter 7 .

\subsubsection{Wettability}

Wettability is a measure of how easily a liquid adheres to a surface. A "wetting" surface will cause a small contact angle, $\theta$ between the liquid and the surface, whereas a "nonwetting" surface will cause a liquid to have a large contact angle with the surface as shown in figure 4.3. The surface will be fully wetting when $\theta=0$ and fully nonwetting when $\theta=\pi$. This contact angle becomes difficult to measure in irregular surfaces such as those found in reservoir rocks and therefore the wettability in these materials cannot be measured directly.

However, one way of measuring wettability in porous media was first proposed by Brown and Fatt in 1956 [59], where the $T_{1}$ of water wet and oil wet sand packs was found to be shorter in water wet systems. If we assume that the water molecules at the surface of the solid are in rapid exchange with those molecules in the bulk fluid, the $T_{1}$ is given by [60]

$$
\frac{1}{T_{1}}=\frac{1-f}{T_{1 b}}+\frac{f}{T_{1 s}}
$$

where $T_{1 b}$ is the relaxation time of the bulk fluid, $T_{1 s}$ is the relaxation time of the fluid at the surface, and $f$ is the fraction of fluid molecules in contact with the 


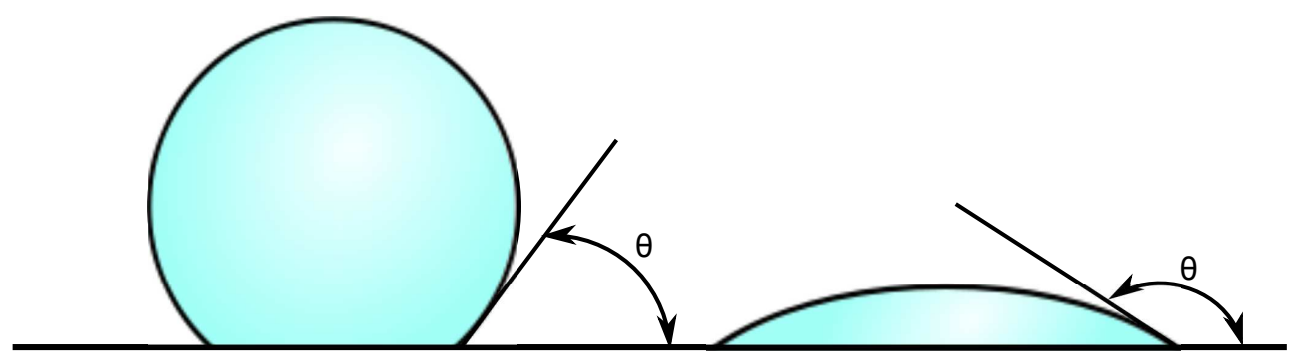

Figure 4.3: The critical angle, $\theta$, and its relationship with wettability. A non-wetting surface will have a small $\theta$ as seen on the left, whereas a wetting surface will have a large $\theta$, as seen on the right.

surface. Typically, $f \ll 1$. Wettability is characterized by the relaxation rate due to surface effects, $1 / T_{1}-1 / T_{1 b}$, a faster relaxation indicates a more water wet surface or a more relaxing surface [61].

\subsubsection{Surface to Volume Ratio and Surface Relaxivity}

We know the average $T_{1}$ in a pore is given by equation 4.5. If the thickness of $f$ is given by $h$, the pore surface area is $S$ and the pore volume is $V$, we can rewrite equation 4.5 as [62]

$$
\frac{1}{T_{1}}=\frac{1}{T_{1 b}}+\frac{S h}{V}\left(\frac{1}{T_{1 s}}-\frac{1}{T_{1 b}}\right)
$$

which reduces to [63]

$$
\frac{1}{T_{1}}=\frac{1}{T_{1 b}}+\rho_{1} \frac{S}{V}
$$

where $\rho_{1}$ is the surface relaxivity for spin-lattice relaxation. The surface relaxivity is a constant and represents the velocity at which the nuclear magnetization leaves the fluid at the pore surface and typically has units in the range of a few $\mu \mathrm{m} / \mathrm{s}$ [64] and can be measured using PFG methods [65, 66]. In the fast diffusion regime, in which molecules diffuse rapidly across local pores in the time $t$, the combination of surface relaxation and restricted diffusion in the presence of a field gradient cause the magnetization decay with respect to $\rho$ during a PFG 
experiment as given by Lipsicas [67]

$$
-\ln \frac{M(t)}{M(0)} \sim \frac{t}{T_{2 B}}+\left(\frac{27}{20} \rho^{2} t^{2} \gamma^{2} \mathbf{g}^{2} \delta^{2}\right)^{1 / 3} .
$$

By plotting $-\ln M(t) / M(0)$ with respect to $(\mathbf{g} \delta)^{2} / 3, \rho$ can be found from the slope.

Note that in the slow diffusion regime, the $T_{2}$ relaxation is dependent on only the internal field gradient rather than both the internal field gradient and surface relaxivity [25]

$$
\frac{1}{T_{2}^{\prime}}=\frac{1}{T_{2}}+\gamma^{2} g^{2} t^{2} \frac{D}{3}
$$

If the value of $\rho_{1}$ or $\rho_{2}$ is known, the surface to volume ratio can be calculated by the rate of $T_{1}$ or $T_{2}$ relaxation. Since the relaxation time of water in the pore space is generally much shorter than that of bulk water we can neglect the bulk first term in equation 4.7:

$$
\frac{1}{T_{1}}=\rho_{1} \frac{S}{V}
$$

Similarly for $T_{2}$

$$
\frac{1}{T_{2}}=\rho_{2} \frac{S}{V} .
$$

If $\rho$ is known, equations 4.10 and 4.11 allow us to use the rate of $T_{1}$ or $T_{2}$ relaxation to find $S / V$. Assuming a spherical pore, the pore radius $a$, is given by $S / V=3 / a$ [68].

Another method of determining the surface to volume ratio involves using the diffusion constant rather than the $T_{1}$ or $T_{2}$ relaxation. In 1992, Mitra et al [69] developed an expression for the surface to volume ratio which is independent of surface relaxivity by utilizing random walk simulations in porous media which allow for surface relaxivity and assume a smooth pore surface. They derive the following equation for short times, $t \rightarrow 0$

$$
\frac{D(t)}{D_{0}}=1-\frac{4}{3 d \sqrt{\pi}}\left(\frac{S}{V}\right)\left(D_{0} t\right)^{1 / 2}+\mathcal{O}\left(D_{0} t\right) .
$$

where $d$ is the spatial dimension. This relationship will be discussed further in section 4.4.1. 


\subsubsection{Pore Size Distribution}

We can define the "pore size" in a sample as a pore with diameter $d$ such that $d$ defines the largest sphere that contains the point of observation inside a pore and is still entirely within the surrounding pore space. If we assign a pore diameter to each pore in the sample we can define a pore size distribution as $\alpha(d)$, where $\alpha$ is the fraction of total pore space volume with a pore diameter between $d$ and $d+\delta d$ where $\lim \delta d \rightarrow 0$. Since the geometric shape of a pore is often hard to define, for simplicity we can envision the pore system as a series of spheres connected by capillary tubes.

Due to the variation in pore sizes in porous materials, there will also be a distribution of relaxation times which from equation 4.11 the magnetization will follow

$$
M(t)=\sum_{i} M_{i} \exp \left[-\rho_{2}\left(\frac{S}{V}\right)_{i} t\right]
$$

where each $i$ represents a pore and where $M_{i}$ is proportional to the volume of fluid in each pore.

The pore size distribution can be obtained by taking an inverse Laplace transform (section 2.9.2) of equation 4.13, and scaling with $\rho_{2}$.

\subsection{Internal Magnetic Fields}

\subsubsection{Internal Magnetic Field Calculations}

As discussed in section 4.2, all porous media are made up of two phases: the solid phase and the fluid, or empty phase. Each of these phases will have an associated magnetic susceptibility, $\chi$, and the difference in $\chi$ between these two phases, $\delta \chi$, will cause inhomogeneities in the internal magnetic field roughly on the order of $\delta \omega=\gamma \delta \chi B_{0}$. This will cause line broadening in the one-dimensional NMR spectrum. This line broadening was first investigated by Brown [70] in 1961, in which he studied the line broadening caused by ferromagnetic micronsized grains suspended in water with 5\% carboxy-methylcellulose. In 1962 this work was expanded upon by Drain [71] who looked at magnetic resonance line broadening in powdered samples. 


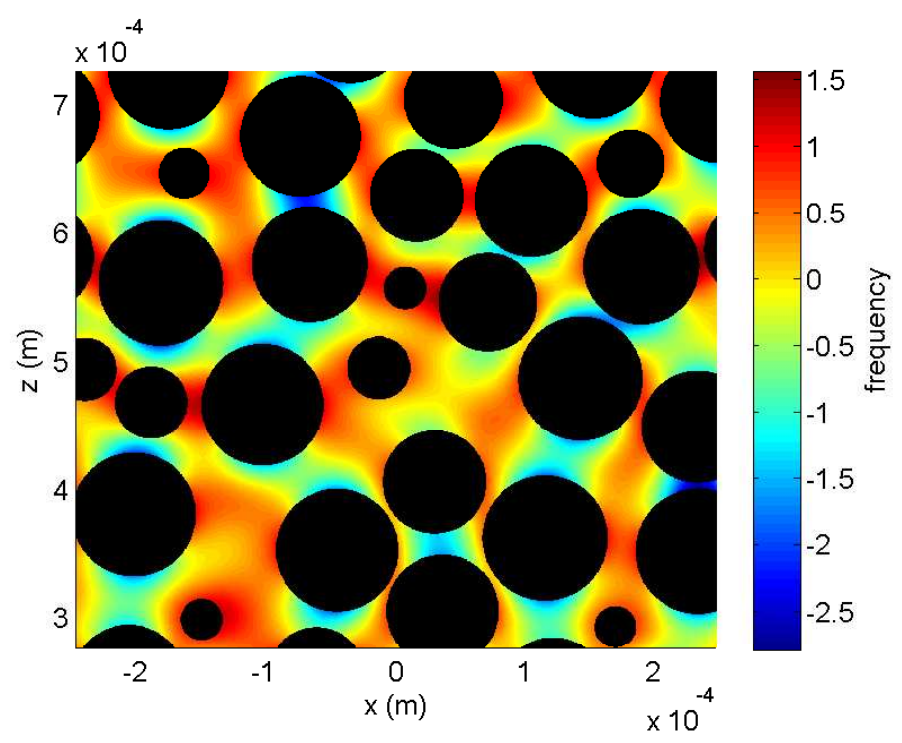

Figure 4.4: Simulated $B_{z}^{i}$ for a random pack of spheres. The spheres have a radius of $48.6 \mu \mathrm{m}$. Details on its calculation are given in section 5.3. Note frequency is in dimensionless units.

We can calculate these internal magnetic fields by first considering a sample porous medium whose matrix is of magnetic susceptibility $\chi_{b}$ and is filled with a fluid of magnetic susceptibility $\chi_{f}$, and then exposed to an external magenetic field, $B_{0}$. The difference in $\chi_{,}\left|\chi_{b}-\chi_{f}\right|=\delta \chi$ will cause pertubations in $\mathbf{B}_{\mathbf{0}}(\mathbf{r})$ at a position $\mathbf{r}^{\prime}$ where the offset, $\Delta \mathrm{B}\left(\mathbf{r}^{\prime}\right)$ is given by

$$
\Delta \mathbf{B}\left(\mathbf{r}^{\prime}\right)=\frac{\mu_{0}}{4 \pi} \int_{V}\left[3 \frac{\mathbf{M}(\mathbf{r}) \cdot\left(\mathbf{r}^{\prime}-\mathbf{r}\right)}{\left|\mathbf{r}^{\prime}-\mathbf{r}\right|^{5}}\left(\mathbf{r}^{\prime}-\mathbf{r}\right)-\frac{\mathbf{M}(\mathbf{r})}{\left|\mathbf{r}^{\prime}-\mathbf{r}\right|^{3}}\right] d r
$$

where $\mathbf{M}(\mathbf{r})$ is the local magnetization given by

$$
\mathbf{M}(\mathbf{r})=\frac{1}{\mu_{0}} \chi(\mathbf{r}) B_{0} \hat{\mathbf{k}}
$$

provided $\chi \ll 1$.

An example of this is shown in figure 4.4.

In this thesis, we are mostly concerned with the internal magnetic field parallel to $B_{0}$, which we label $B_{z}^{i}$.

The value of $B_{z}^{i}$ is dependent on the pore shape and size and has been studied by various groups. Sen and Axelrod [72] examined the positional dependence of $B_{z}^{i}$ in an array of cylinders assuming a point dipole at the center of 
each cylinder and used a combination of a position dependent discrete sum inside a Lorentz cavity and an integral outside the cavity. They find that $B_{z}^{i}$ varies over the length scale of the pores. Cho [73] used MRI on an array of cylinders and found similar results to Sen.

Audoly et al. [74] examine the structure of the $B^{i}$ field in a dense random pack of non-penetrating spheres. They treat each bead as a dipole and calculate the total field in the bead pack as a superposition of the fields from each sphere. Similarly to Sen et al, they find that that the variation of $B_{z}^{i}$ occurs over the length of a pore. Plotting a distribution of the $B_{z}^{i}$ field shows a nearly symmetrical distribution showing an average $B_{z}^{i}$ field of zero across the bead pack due to the isotropy of the randomly packed beads. The maximum $B_{z}^{i}$ occurs where there are two beads close to one another that are also parallel to the applied external magnetic field, $B_{0}$. Minimum $B_{z}^{i}$ occurs where two beads are close to one another perpendicular to $B_{0}$.

Chen et al. [75] created a simulation of the internal magnetic fields in a fully water saturated and partially water and air saturated Berea sandstone based on the pore structure of a thin slice of Berea. They find results similar to Audoly [74] that the internal field is proportional to $B_{0}$ and $\Delta \chi$ and that the $B^{i}$ variation occurs over the length of a pore. They also find that the $B^{i}$ distribution is similar for both fully and partially saturated pores.

\subsubsection{Relaxation Due to Diffusion in Internal Magnetic Fields}

Molecular diffusion through the inhomogeneous field will cause irreversible dephasing (i.e. phase change from an echo) which will cause an increase in transverse relaxation such that $T_{2} \ll T_{1}$. The change in transverse relaxation is dependent on the local frequency fluctuations, $\Delta \omega_{0}(t)$, which can be characterized by defining a correlation time

$$
\tau_{c}=\int_{0}^{\infty} \frac{\left\langle\Delta \omega_{0}(t+\tau) \Delta \omega_{0}(t)\right\rangle}{\left\langle\Delta \omega_{0}^{2}\right\rangle} d \tau
$$

where $\left\langle\Delta \omega_{0}^{2}\right\rangle$ is the second moment of the linewidth as $\tau \rightarrow \infty$. We also use

$\tau_{c}$ to define "rapid fluctuations" and "slow fluctuations" as $\tau_{c}^{-1} \gg \sqrt{\left\langle\Delta \omega_{0}^{2}\right\rangle}$ 

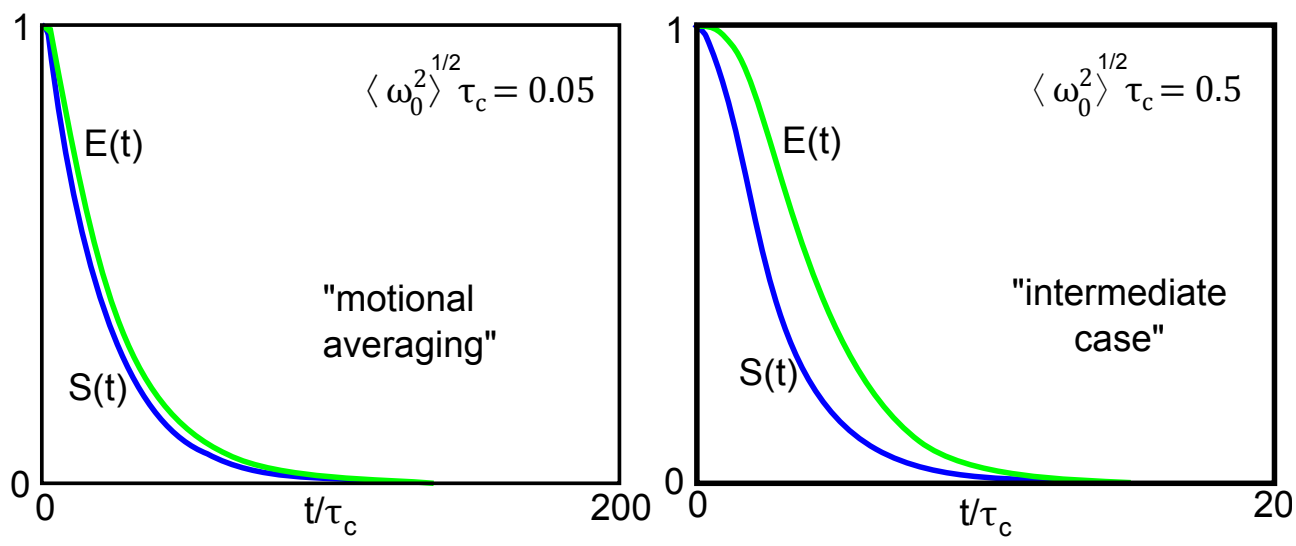

Figure 4.5: $S(t)$ and $E(2 \tau)(E(2 \tau)=E(t))$ in the fast (left) and intermediate (right) fluctuation regimes. Adapted from [4].

and $\tau_{c}^{-1} \ll \sqrt{\left\langle\Delta \omega_{0}^{2}\right\rangle}$ respectively. Rapid fluctuations will produce homogeneous broadening which is irreversible and decays at a relaxation time, $T_{2}$, of $\left(\left\langle\omega_{0}^{2}\right\rangle \tau_{c}\right)^{-1}$. The slow fluctuations produce inhomogeneous broadening which is reversible and,using a Gaussian assumption, decays at a relaxation time $T_{2}^{*}$, of $\exp \left(-\frac{1}{2}\left\langle\Delta \omega_{0}^{2}\right\rangle t^{2}\right)$

We can determine the behavior of the FID and spin echo decays by using the Anderson-Weiss theory [76] and the fluctuating field correlation function

$$
g_{\omega}(\tau)=\frac{\left\langle\Delta \omega_{0}(t+\tau) \Delta \omega_{0}(t)\right\rangle}{\left\langle\Delta \omega_{0}^{2}\right\rangle} .
$$

For random processes this correlation is typically given by

$$
g_{\omega}(\tau)=\exp \left(-|\tau| / \tau_{c}\right)
$$

This will give us an FID decay, $S(t)$ and spin echo amplitude, $E(2 \tau)$ of [3]

$$
\begin{aligned}
& S(t)=\exp \left(-\left\langle\Delta \omega_{0}^{2}\right\rangle \tau_{c}^{2}\left(\exp \left(-t / \tau_{c}\right)-1+t / \tau_{c}\right)\right) \\
& E(2 \tau)=\exp \left(-\left\langle\omega_{0}^{2}\right\rangle \tau_{c}^{2}\left(4 \exp \left(-\tau / \tau_{c}\right)-\exp \left(-2 \tau / \tau_{c}\right)+2 \tau / \tau_{c}-3\right)\right)
\end{aligned}
$$

Figure 4.5 shows equation 4.19 in both the slow and intermediate fluctuation regimes. We define the relaxation times at these regimes as $T_{2}^{*}$ and $T_{2}^{\dagger}$, respectively [77].

The internal magnetic field inhomogeneities can be used to characterize the pore space of a sample. In 2000, Song et al [78] developed a method to obtain 
characteristic pore sizes and pore size distributions in porous materials such as glass bead packs and sandstone and carbonate rocks using the $B^{i}$ fields generated by susceptibility differences. They used a combination of the echo signal, $E$, obtained from applying a stimulated echo and the initial amplitude of an FID signal, $R$, where the phase contribution from the $B^{i}$ field has been eliminated. By combining these two signals, they found the signal decay produced from the $B^{i}$ field alone. By plotting the ratio of $E / R$ to the mixing time used in the stimulated pulse sequence, and applying an inverse Laplace transform, Song was able to obtain a pore size distribution for the sample.

Another method to approximate pore size using internal fields is demonstrated by Cho [79] in 2008. They use pulsed field gradient NMR to obtain propagators for which the internal field is nullified, $P$, and for which the internal field is allowed to effect the stimulated echo, $P^{\prime}$. Experiments are performed on two samples of randomly packed monodisperse glass beads. By plotting the ratio of the two propagators, $P / P^{\prime}$ with $Z$-displacement, Cho found that a minimum occurs at $z \sim 1.5 r_{0}$ where $r_{0}$ is the radius of a glass bead. These experimental results are compared with a numerical simulation that treated the field as a superposition of dipoles which are located at the center of each bead. They found reasonable agreement with experiment.

\subsubsection{Gradients Due to Internal Magnetic Fields}

We can also characterize inhomogeneities in $B_{z}^{i}$ by studying the magnitude of the local magnetic field gradient, $\mathrm{g}=\left|\nabla B_{z}^{i}\right|$. Like the internal field, $\mathrm{g}$ scales with the linewidth, $\gamma \delta \chi B_{0}$, but the largest gradients, $g_{\max }$ are found at the pore surface [80,75], and are generally proportional to $\left(\Delta \chi B_{0}\right)^{3 / 2}$ [81]. The value of $g_{\max }$ can also give us information about the smoothness of the pore walls, where a large value of $g_{\max }$ indicates a rough pore surface [82].

Sun and Dunn [83] correlate internal field gradients with $T_{2}$ relaxation in two different sandstone samples of varying permeabilities. Using the assumption that a short $T_{2}$ time corresponds to a small pore size, they find that gradient strengths are highest in smaller pores. 


\subsection{Diffusion in the Pore Space}

Diffusion is first discussed in section 3.2.6, where we first defined the diffusion constant $D$ as a function of the r.m.s. displacement and diffusion time. When we place the bulk fluid in a restrictive medium, such as a pore space, the diffusion of the molecules within this geometry is inhibited by the walls of the pore, which means we cannot assign a single diffusion coefficient to this behavior, but we must use a time-dependent value of diffusion, $D(t)$, which can give us information about the pore structure. Restricted diffusion was first studied by Woessner in 1962 [84] where he studied the $D(t)$ of water and silica suspensions, water in sandstone, and benzine imbibed into rubber. A very good tutorial on using $D(t)$ to study pore geometry is written by Sen [85]. Callaghan's books [3, 4] are also recommended resources for information about restricted diffusion.

We can define $D(t)$ using the mean squared displacement given by Einstein [86] as

$$
D(t)=\frac{\left\langle(\mathbf{r}(t)-\mathbf{r}(0))^{2}\right\rangle}{6 t}
$$

where $\mathbf{r}$ is the position of a molecule. If we take the limit of $t \rightarrow 0, D(t)$ will approach the same value as that of bulk diffusion, $D_{0}$ since with a smaller timestep, the molecules are less likely to encounter a pore wall, therefore for short diffusion times, $\Delta, D(t)$ will be similar to the bulk diffusion coefficient, $D_{0}$. How short $\Delta$ needs to be will be dependent on the pore geometry. Given a characteristic structural length, $l$, which is the mean distance between the pore walls, unrestricted diffusion will occur at $\sqrt{D_{0} \Delta} / l \ll 1$, and if $\sqrt{D_{0} \Delta} / l \gg 1, D(t)$ will become asymptotic.

\subsubsection{Diffusion in the Short Time Limit}

From the short-time limit, we can get information about the surface to volume ratio of the pore space. During short times, $\sqrt{D t} \ll l$, the only molecules that will come into contact with the wall are those that are close to the surface. The thickness of this layer is on the order of the root mean squared diffusive distance which is $\sqrt{D_{0} t}$. Given the total surface area exposed to these molecules, $S$, the total volume of molecules within this is given by $S \sqrt{D_{0} t}$. Therefore the vol- 
ume fraction over the entire pore space for these surface molecules is given by $S \sqrt{D_{0} t} / V$ where $V$ is the total pore volume. We can then assume that $D(t) / D_{0}$ will be given by

$$
\frac{D(t)}{D_{0}}=1-\frac{S \sqrt{D_{0} t}}{V}
$$

From this, Mitra et al $[69,87]$ derive $D(t)$ for short times

$$
\frac{D(t)}{D_{0}}=1-\frac{4}{9 \sqrt{\pi}} \frac{S}{V} \sqrt{D_{0} t}-\frac{S}{12 V}\left\langle\frac{1}{R_{1}}+\frac{1}{R_{2}}\right\rangle D_{0} t+\frac{1}{6} \frac{\rho S}{V} D_{0} t+\mathcal{O}\left[\left(D_{0} t\right)^{3 / 2}\right] .
$$

where $R_{1}$ and $R_{2}$ are the principal radii of curvature over the pore surface, and $\rho$ is the surface relaxivity. For a flat reflecting wall, equation 4.22 simplifies to 4.12 .

In figure 4.6 we show an example of the change in $\left\langle(\mathbf{r}(t)-\mathbf{r}(0))^{2}\right\rangle / 6$ with respect to $l$ and $\Delta$. We use a simulated random pack of spheres and vary the sphere radius, $R$, to increase or decrease $l$. Larger values of $R$ will result in smaller values of $l$. Free diffusion, $D_{0}$, is given by the dashed line.

\subsubsection{Diffusion in the Long Time Limit}

As $t \rightarrow \infty$ in well-connected porous media, $D(t)=D_{0} / \alpha$, where $\alpha$ is the tortuosity as described in section 4.2.3. In simple systems such as a random bead pack, $\alpha=\sqrt{\phi}$ [58]. In more complicated structures, from equation 4.3, $D(t)=D_{0} / F \phi$.

We can also get information about the pore space from how the long-time limit $D_{0} / \alpha$ is reached. de Swiet and Sen [88] found that in general, for a porous medium with non-penetrating walls, for long times $\sqrt{D t} \gg l$, the diffusion coefficient will approach its long-time limit as

$$
\frac{D(t)}{D_{0}} \rightarrow \frac{1}{\alpha}+\frac{\beta}{D_{0} t}-\frac{\gamma}{\left(D_{0} t\right)^{3 / 2}}+\ldots \text { as } t \rightarrow \infty
$$

where $\alpha, \beta$, and $\gamma$ are dependent on the pore geometry and cannot be predicted as part of a general theory. 


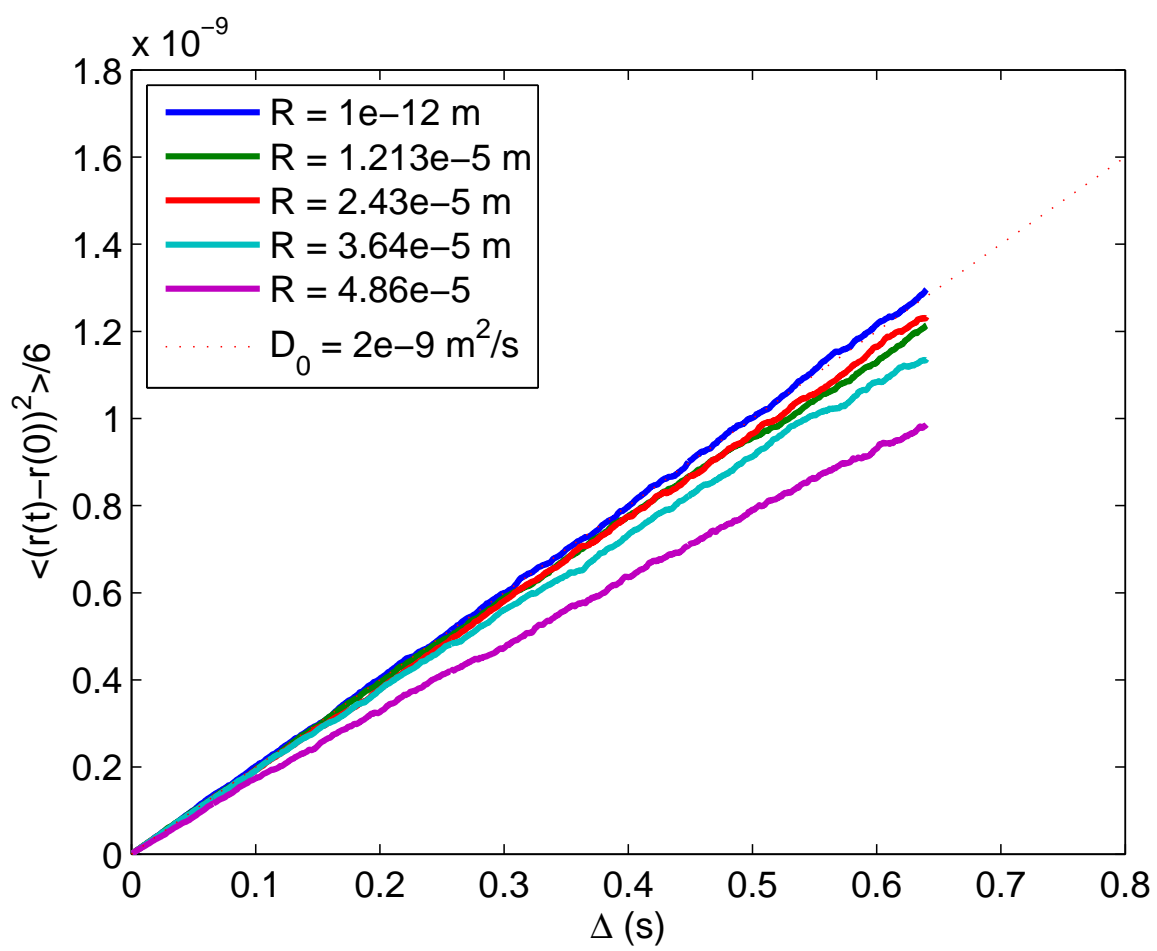

Figure 4.6: $\left\langle(\mathbf{r}(t)-\mathbf{r}(0))^{2}\right\rangle / 6$ in the short time limit with respect to $l$ and $\Delta$. Data is generated using a random walk through a matrix of randomly packed spheres. To decrease $l$, the sphere radius, $R$ is increased resulting in a greater deviation of $\left\langle(\mathbf{r}(t)-\mathbf{r}(0))^{2}\right\rangle / 6$ from the free diffusion curve as given by the dashed line or the solid blue line where $R=10^{-12} \mathrm{~m}$. 


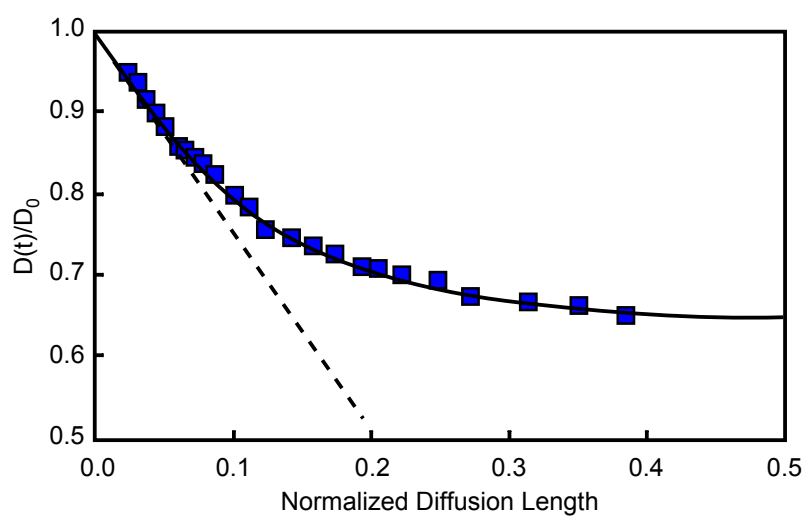

Figure 4.7: $D(t) / D_{0}$ for $4 \mathrm{~mm}$ beads with a Xe gas pressure of 6.41 bar. The solid line is the Padé approximation given by equation 4.24. The dashed line shows the short-time diffusion behavior as given by $\sqrt{D_{0} t}[69,87]$. Adapted from reference [91].

\subsubsection{Diffusion in the Intermediate Time Limit}

To interpolate between the short and long time diffusion regimes we make use of the two-point Padé approximation $[89,90]$

$$
\frac{D(t)}{D_{0}}=1-\left(1-\frac{1}{\alpha}\right) \frac{c \sqrt{t}+(1-1 / \alpha) t / \theta}{(1-1 / \alpha)+c \sqrt{t}+(1-1 / \alpha) t / \theta}
$$

where $c=(4 / 9 \sqrt{\pi})(S / V) \sqrt{D_{0}}$ and $\theta$ has dimensions of time. In bead packs, $\theta$ will be proportional to the pore separation, $b$. For general porous media, $\theta$ has not been found to have a geometrical equivalent but is used as fitting parameter. Mair et al [91] performed a series of experiments of xenon gas diffusion through random packs of monodisperse glass beads. $D(t) / D_{0}$ for a sample of diameter $4 \mathrm{~mm}$ under a Xe gas pressure of 6.41 bar is shown in figure 4.7

The change in mean squared displacement over time will also have an effect on the shape of the propagator. Propagators are first introduced in section 3.2.9 and are the probability a molecule has diffused a distance $R$ during time $t$. In free diffusion, the propagator is Gaussian in shape, however, with restrictive diffusion, the propagator's shape may become time dependent depending on the pore geometry. Tanner [92] calculated $D(t)$ for a system comprised of a series of permeable barriers and found that the propagator would cease to be gaussian at $t \sim a^{2} / D$ where $a$ is the pore radius. 


\subsubsection{Diffusive Diffraction}

Given an ensemble of molecules in a single pore, as $t \rightarrow \infty$, the starting point of molecules in the pore space will become unimportant as molecules will be found everywhere in the pore no matter what their starting point. This will cause $P(\mathbf{r} \mid \mathbf{r}, \Delta)$ to reduce to the pore molecular density function, $\rho\left(\mathbf{r}^{\prime}\right)$. The average propagator will become

$$
\overline{P_{s}}(\mathbf{R}, \infty)=\int \rho(\mathbf{r}+\mathbf{R}) \rho(\mathbf{r}) d \mathbf{r}
$$

which is the autocorrelation function of $\rho(\mathbf{r})$. The Wiener-Kintchine theorem [93], which states that the Fourier transform of an autocorrelation function is the frequency power spectrum, thus

$$
E_{\infty}(\mathbf{q})=|S(\mathbf{q})|^{2}
$$

where $q$ is the reciprocal space defined by equation 3.13. Cory and Garroway [94] pointed out that this result is analogous to the echo amplitude being equivalent to the diffraction pattern produced by a single slit in optics.

If we expand this single pore system to a multi-pore system with interconnected pores that allow the molecules to diffuse to different pores, this single slit experiment analogy will expand to a multiple slit experiment where the elements of the "diffraction grating" are defined by probability of a molecule diffusing to a different pore during $\Delta$ [95]. A good example of this "diffraction effect" is from Callaghan et al [95] where they perform a series of PGSE experiments on a loose randomly packed glass bead matrix of pore spacing $15.8 \mu \mathrm{m}$ at $\Delta$ times ranging from 20 to $110 \mathrm{~ms}$. They find a coherence peak at $q \approx(16 \mu \mathrm{m})^{-1}$ for values of $\Delta \geq 70 \mathrm{~ms}$ as shown in figure 4.8 .

\subsection{This Thesis}

In this thesis we seek to characterize porous media by exploiting the inhomogeneous internal magnetic field generated by susceptibility differences between the solid matrix and fluid filled pores as discussed in section 4.3.1 of this chapter. We will use three different experiments to achieve this goal: the first is frequency 


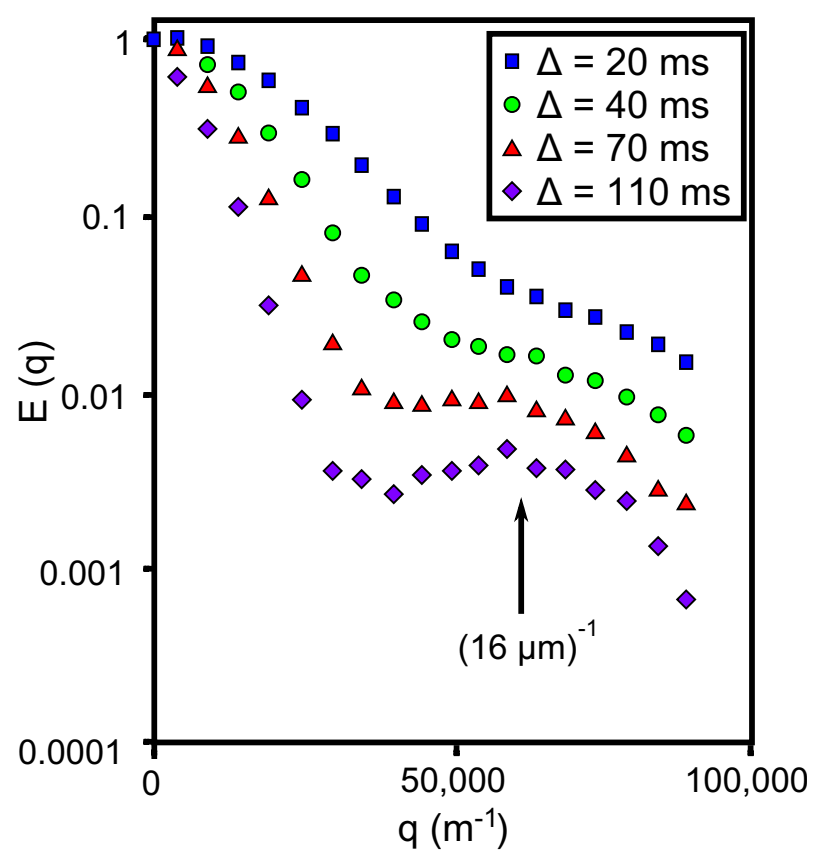

Figure 4.8: Echo intensities $E(q)$ for a glass bead matrix of pore spacing $15.8 \mathrm{mu} \mathrm{m}$. A coherence peak is located at $q \approx(16 \mu \mathrm{m})^{-1}$ for values of $\Delta \geq 70 \mathrm{~ms}$.Adapted from reference [95]. 
exchange where use a two-dimensional exchange experiment to separate the line broadening caused by the susceptibility difference in a monodisperse bead pack and the line broadening caused by diffusion through the inhomogeneous field. For our second experiment, we expand upon the first experiment and add a third dimension in the form of a propagator. What the propagator dimension will allow us to do is separate exchange spectra by the $Z$-displacement of the spins in the pore space of the system. Finally, we move away from exchange experiments and switch to a correlation experiment, where we correlate the transverse relaxation decay, $T_{2}$ with the inhomogeneous magnetic field, and vary the echo spacing between pulses.

Before we describe our experiments, however, we first want to review the development of a random-walk simulation specifically developed for these experiments. We use this simulation not only to simulate the exchange and correlation spectra, but to simulate the displacement propagators and visualize the internal magnetic field and the internal gradient field. 


\section{Chapter 5}

\section{Simulation}

\subsection{Introduction}

An important part of this thesis concerns creating software which we can use to simulate our multi-dimensional NMR experiments. Since all our experiments will involve diffusion through an inhomogeneous magnetic field, we require that our program calculate an internal magnetic field and also simulate diffusion. To do these things, we first generate a dense random pack of spheres, place a point dipole aligned along the $z$-direction at the center of each sphere then calculate the internal magnetic field in the void space as a superposition of all dipoles in the system. The magnetic field outside a charged sphere is exactly the same as that outside a point dipole [97], and as we are only concerned with the field in the pore space, using a superposition of point dipoles in the bead pack will give us an accurate representation of the internal field. We look at two different methods of simulating diffusion, both random walk methods; but one method restricts the walk to discrete jumps along the $x y z$-axes, while the other allows for a random step at any orientation. Later on we also add a basic method to include $T_{2}$ relaxation within the simulation. 


\subsection{Creating the Bead Pack}

To generate our random bead pack we use an algorithm written by Jackson [96] in which we define a cylinder of diameter $D_{c y l}$ and length $L_{c y l}$, whose bottom face is centered at the origin and the cylinder is aligned along the $z$-axis. Then spheres of radius $R_{\text {sphere }}$ are randomly packed inside the cylinder by selecting a random point at $z=0$ and then random walking mostly in the $+z$ direction until the sphere is outside the rest of the bead pack. The sphere will then follow a downwards random walk until it reaches a local potential energy minimum. Essentially, the beads are dropped one by one into a cylinder. This will result in a list of coordinates for the beadpack in units of sphere diameter.

For the beadpack used in this simulation, we use a cylinder of height $20 R_{\text {sphere }}$ and with a radius of $10 R_{\text {sphere }}$. This beadpack is shown in figure 5.1.
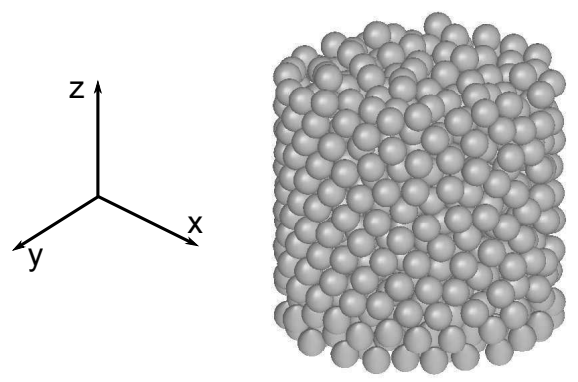

Figure 5.1: The actual beadpack used in the simulation. The cylinder has a radius of $10 R_{\text {sphere }}$ and a height of $20 R_{\text {sphere }}$.

\subsection{Simulating the Internal Magnetic Field}

To simulate our internal magnetic field, $B_{z}^{i}$, we place a magnetic dipole at the center of each sphere and align it with the $z$-axis. This method has been used previously by Audoly [74]. The magnetic field at a distance $\mathbf{R}$ from a dipole with magnetic moment $\mathbf{m}$ is given by equation 5.56 in Jackson [97]

$$
\mathbf{B}_{\mathbf{z}}^{\mathbf{i}}(\mathbf{r})=\frac{\mu_{0}}{4 \pi}\left[\frac{3 \mathbf{n}(\mathbf{n} \cdot \mathbf{m})-\mathbf{m}}{|\mathbf{r}|^{3}}\right]
$$


where $\mathbf{n}$ is the unit vector in the direction of $\mathbf{r}$. The magnetic field of the $z$ component is given by

$$
B_{z}^{i}(r) \hat{z}=\frac{\mu_{0}}{4 \pi} \frac{m\left(3 \cos ^{2} \theta-1\right)}{|\mathbf{r}|^{3}}=\frac{\mu_{0}}{4 \pi} m\left[\frac{3 z^{2}}{r^{5}}-\frac{1}{r^{3}}\right]
$$

where $m=|\mathbf{m}| \propto R_{\text {sphere }}^{3}, r=|\mathbf{r}|$, and $\theta$ is the polar inclination angle from the dipole to the point of measurement.

These points of measurement will be our tracer points. Initially, these points were placed in the pore space along a $120 \times 120 \times 120$ mesh cube with a side of approximately 4 bead diameters which was centered inside the bead cylinder. The size of this cube was chosen so as to ensure no tracers would diffuse outside the bead pack. This was a rather simplistic method, and has since been upgraded so that tracers are placed randomly through the pore space of the bead pack rather than along a set mesh cube. First we place a large number of tracers, $N_{\text {tracer }}$, typically on the order of $2.5 \times 10^{6}$, inside our cylindrical boundary which is defined as having a radius 1 sphere diameter smaller than that of the bead pack. The reason for this is to eliminate edge effects which will be discussed in further detail later. Given a cylinder which is aligned along the $z$ axis, the cylindrical coordinates, $\left(r_{\text {trac }}^{i}, \theta_{t r a c}^{i}, z_{t r a c}^{i}\right)$ for each of the tracer particles $i=1, \cdots, N_{\text {tracer }}$ are given by

$$
\begin{aligned}
r_{\text {trac }}^{i} & =r_{\text {max }} * \text { rand }^{i} \\
z_{\text {trac }}^{i} & =z_{\text {min }}+\text { rand }^{j} * z_{\text {max }} \\
\theta_{\text {trac }}^{i} & =2 \pi * \text { rand }^{k}
\end{aligned}
$$

where $r_{\max }, z_{\min }$, and $z_{\max }$ are defined by the boundary conditions, and the rand $^{i, j, k}$ comprise $1 \times N_{\text {tracer }}$ vectors of uniformly distributed random numbers between 0 and 1 .

After we have placed our tracers inside our selected boundary conditions, we check each one to make sure it is not inside a bead by comparing each tracer position with the bead coordinates. If a tracer is within a bead radius of a bead center coordinate, that tracer is dropped. We can find the simulated sphere pack porosity, $\phi_{\text {pack }}$ by $N_{\text {pore }} / N_{\text {tracer }}$ where $N_{\text {pore }}$ is the number of tracers in the pore space of the sphere pack. 
Now that we have our tracers randomly placed in the pore space, we can use these as our points of measurement for equation 5.2. From the values of $B_{z}^{i}$ for each tracer, we can calculate the 1D spectrum of the field as shown in figure 5.2. The resultant field map, $B_{z}^{i}$ is shown in figure 5.3. In contrast to how we calculate our 2D spectra (described at the end of the chapter), which uses tracers that are randomly placed in the pore space of the bead pack, the magnetic field (and later, gradient) maps are generated using tracers placed along a 1000 $\times 1000$ mesh which is centered at $y=0$ and aligned along the $x z$-plane. Only tracers on the mesh which are located in the pore space are used.

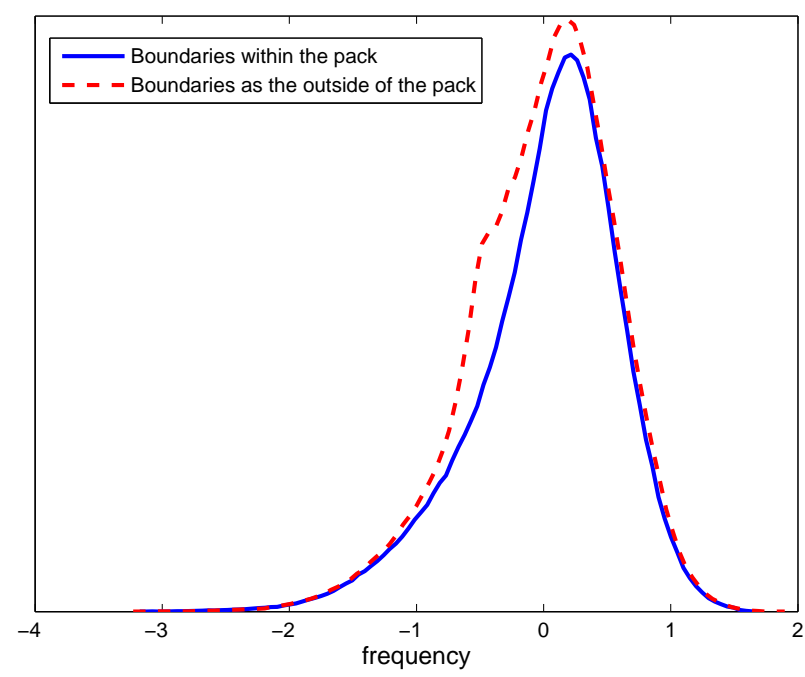

Figure 5.2: Effects of boundary condition choice on the 1D spectrum. The solid blue line shows the 1D spectrum if boundary conditions are chosen to be within a single bead diameter of the outside of the cylindrical bead pack. The dashed red line is the 1D spectrum if boundary conditions are selected to be the outer edge of the cylindrical bead pack. Note that the frequency is dimensionless.

In this calculation, $\chi<0$, therefore the strongest negative field is found at the $z$-poles, while the strongest positive field is found around the equator of the beads. This field distribution is due to the fact we have a dipole oriented along the $z$-direction at the center of each sphere.

At this point it is important to discuss the importance of selecting bound- 


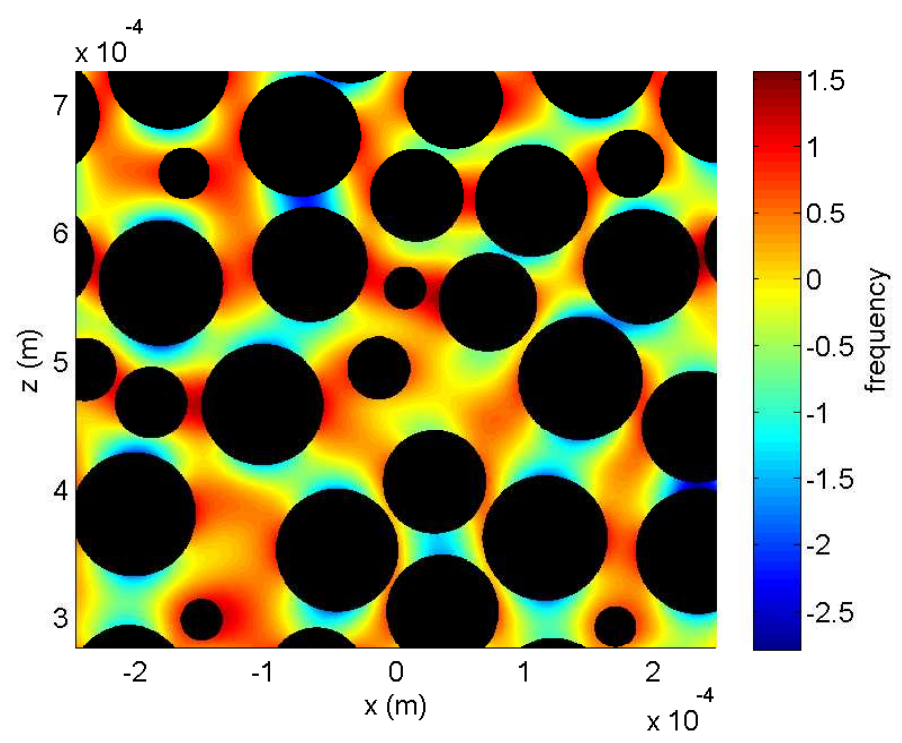

Figure 5.3: The internal magnetic field. A $1000 \times 1000$ mesh is centered at $y=0$ and aligned along the $x z$-plane. Tracers are placed on the mesh in the pore space and the internal field at that point is calculated using equation 5.2. The spheres in this figure have a radius of $48.6 \mu \mathrm{m}$.

ary conditions that incorporate enough of the sample to produce meaningful results, while being small enough to avoid edge effects from the outside of the bead pack. If we define the boundary conditions as the edges of the cylinder, we see in the 1D spectrum a negative shoulder as shown by the dashed red line in figure 5.2. Defining the boundary conditions as a bead diameter less than the total cylindrical diameter and height, we find the 1D spectrum no longer has the shoulder as shown in figure 5.2. For best results, we seek to maximize the volume of bead pack simulated while not setting the boundary conditions so large that we have significant edge effects in our simulated data, therefore we set the boundary conditions to be within a bead diameter of the edge of the bead pack. We find a slight negative skew and an average field value of -0.0146 . This is most likely due to edge effects from the size of the simulated bead pack. In an infinite bead pack, we would expect the average field value to be zero.

In figure 5.4 we correlate the internal field with tracer position. At left is the $B_{z}^{i}-z$ correlation, and at right is the $B_{z}^{i}-r$ correlation. The $B_{z}^{i}-z$ correlation shows 

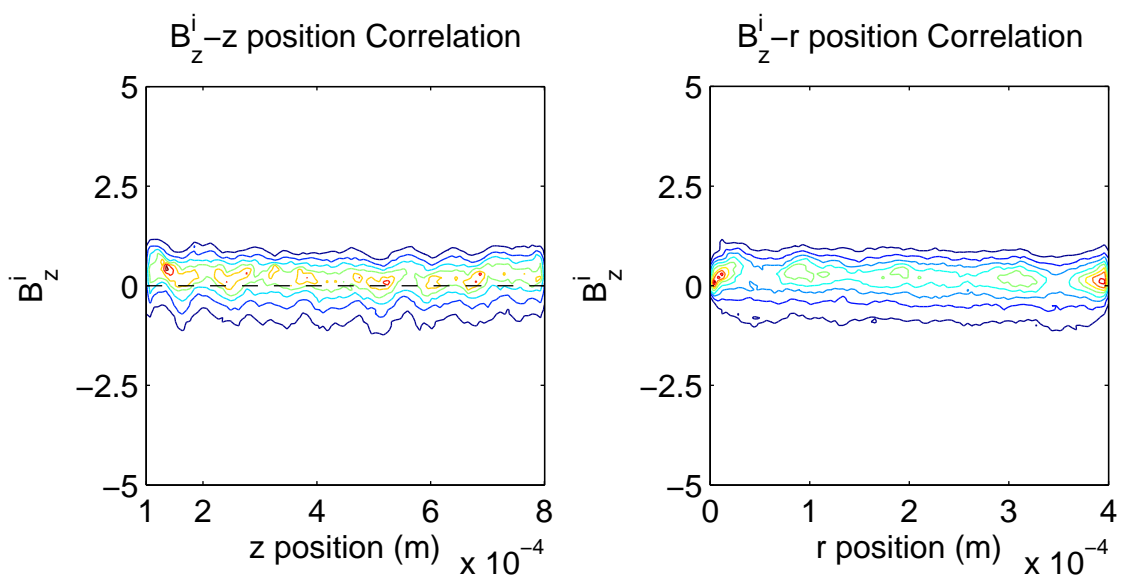

Figure 5.4: The correlations between the internal magnetic field and position. Left: The internal magnetic field and $z$ position correlation. Right: The internal magnetic field and $r$ position correlation. Contour lines range from 200 to 1800 with intervals of 200. Red contours indicate higher intensity. 
that the negative field is sensitive to the vertical spacing of the beads, and at low values of $z$, we see a slight oscillation at negative offsets. This oscillation is likely due to a regularity in the spheres due to the way the sphere pack is constructed with a bottom layer of spheres that rest along a flat bottom parallel to the $x y$-plane at $z=0$. We also note that at $r=r_{\max }$, there is more radial ordering, which may affect the resultant simulated field. Indeed, we see a slight increase towards more positive magnetic field offsets at large values of $r$. The use of an infinite pack would eliminate the chance of ordering along the edges affecting the calculation of the internal magnetic field.

\subsection{Simulating the Magnetic Field Gradient}

The internal magnetic field gradient describes the change in internal magnetic field over a specific distance. Given information about position and the corresponding magnitude of the internal magnetic field at that position, we can calculate the values for the internal magnetic field gradient inside the pore space of the bead pack.

The magnetic field gradient magnitude is given by the equation

$$
\left|\nabla B_{z}^{i}\right|=\sqrt{\left(\frac{\partial B_{z}^{i}}{\partial x}\right)^{2}+\left(\frac{\partial B_{z}^{i}}{\partial y}\right)^{2}+\left(\frac{\partial B_{z}^{i}}{\partial z}\right)^{2}} .
$$

Numerically simulating $\left|\nabla B_{z}^{i}\right|$ requires an additional 6 points per tracer placed along the $x-, y-$, and $z$-axis on either side of the tracer. Using these points we can solve equation 5.4 as the average of $B_{z}^{i+}$ and $B_{z}^{i-}$ where $B_{z}^{i+}$ and $B_{z}^{i-}$ are the value of the internal magnetic field at the points above and below the tracer point along the $x-, y-$, and $z-$ axis. These values are given by

$$
\begin{aligned}
& \left|\nabla B_{z}^{i+}\right|=\sqrt{\left(\frac{B_{z}^{i+}-B_{z}^{i}}{x^{+}-x}\right)^{2}+\left(\frac{B_{z}^{i+}-B_{z}^{i}}{y^{+}-y}\right)^{2}+\left(\frac{B_{z}^{i+}-B_{z}^{i}}{z^{+}-z}\right)^{2}} \\
& \left|\nabla B_{z}^{i-}\right|=\sqrt{\left(\frac{B_{z}^{i}-B_{z}^{i-}}{x-x^{-}}\right)^{2}+\left(\frac{B_{z}^{i}-B_{z}^{i-}}{y^{-}-y^{-}}\right)^{2}+\left(\frac{B_{z}^{i}-B_{z}^{i-}}{z-z^{i}}\right)^{2}}
\end{aligned}
$$

where $x^{+}, y^{+}$, and $z^{+}$are the coordinate values of the points above the tracer along the $x y z$-axes and $x^{-}, y^{-}$, and $z^{-}$are the coordinate values of the points 
below the tracer along the $x y z$-axes. The resultant gradient map for points at a distance of $0.1 R_{\text {sphere }}$ from the tracer are shown as the left plot in figure 5.5.

The addition of 6 points for every tracer in the simulation will cause an increase in calculation time. Also, determining what distance from the tracer is the best choice for calculation introduces another variable to the simulation. A distance too close or too far from the tracer will give an unrealistic representation of the gradient field. Rather than calculating the value of $\left|\nabla B_{z}^{i}\right|$ numerically, we can also calculate it analytically by combining equations 5.4 and 5.2. We obtain

$$
\left|\nabla B_{z}^{i}\right|=\frac{\mu_{0}}{4 \pi} \sqrt{\left(3 x m\left[\frac{5 z^{2}}{r^{7}}-\frac{1}{r^{5}}\right]\right)^{2}+\left(3 y m\left[\frac{5 z^{2}}{r^{7}}-\frac{1}{r^{5}}\right]\right)^{2}+\left(3 z m\left[\frac{5 z^{2}}{r^{7}}-\frac{3}{r^{5}}\right]\right)^{2}}
$$

Note that the magnitude of the internal magnetic field gradient is also given by the notation $|g|$. The results for the analytically calculated gradient are shown in the right plot in figure 5.5. Note that the results for the analytic and numeric gradient are indistinguishable from one another.
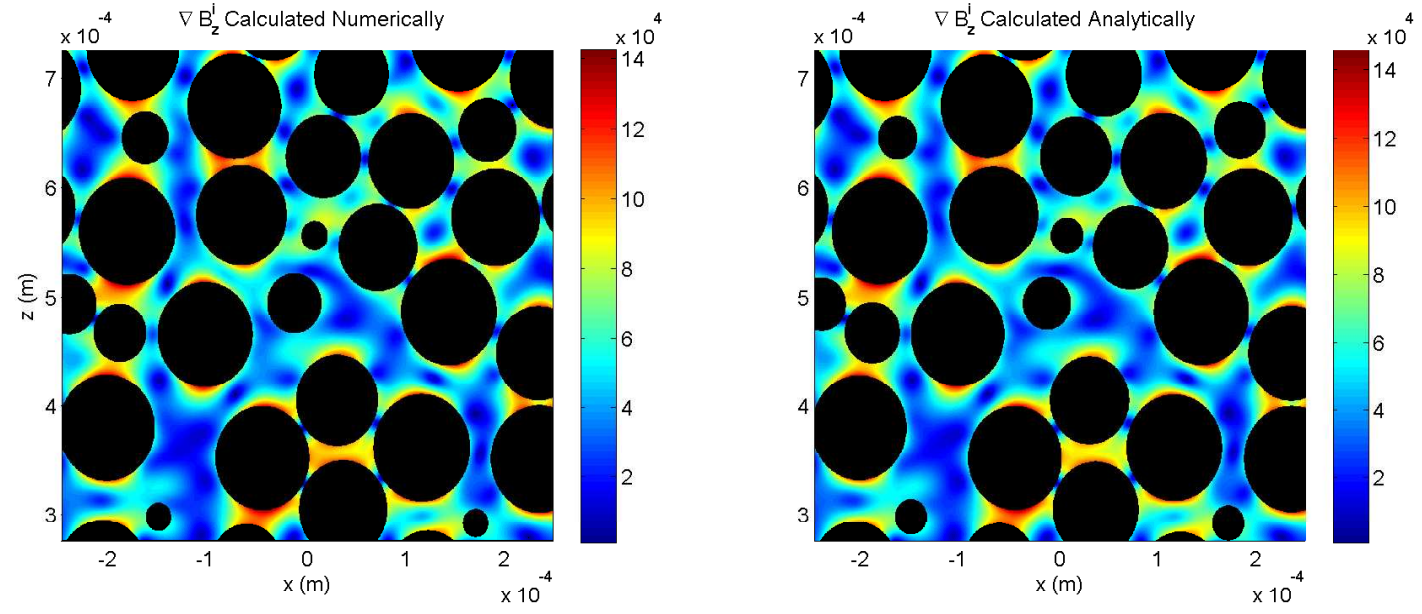

Figure 5.5: Left: Internal field gradient map calculated numerically using equation 5.5. The points $x^{+}, y^{+}$, etc are at a distance of $0.1 R_{\text {sphere }}$ from each tracer. Right: Internal field gradient map calculated analytically using equation 5.6. The color scale represents gradient magnitude. 


\subsection{Random Walk Along $x y z$-axes}

The core of our simulation is the random walk procedure which we use to simulate the diffusion of our spins through the bead pack. We first start out with a simple random walk in which we step a set length $L$ along either the $x-, y-$, or $z$ - directions. In these early stages, $L$ is set to be a fraction of the bead diameter. The change of position for each tracer during each diffusion step is given by

$$
\begin{aligned}
& \Delta x=+L \text { or }-L \\
& \Delta y=+L \text { or }-L \\
& \Delta z=+L \text { or }-L .
\end{aligned}
$$

If a tracer encounters a sphere at any point during the random walk, the last step is thrown out and the final step location will be the same as the initial step location. The step length is calculated from $\langle L\rangle=2 D t$.

To calculate magnetic frequency exchange, this simple simulation is sufficient. However, with the addition of a propagator dimension, the shortcomings of this method soon become apparent.

As defined in section 3.2.9, the propagator is the probability a molecule has diffused a distance $R$ during time $\Delta$. In our simulation, we are concerned with the $Z$-displacement of the tracers in the system, so this method will produce two problems:

1. Since we are only concerned with the $Z$-displacement, having a step that is only along the $z$-axis results in a $Z$-displacement that is discretized to 土step length.

2. For lower number of steps, this discrete step length is glaringly obvious.

Figure 5.6 shows the propagators generated for an increasing number of diffusion steps, $j$. For $j=1$, which is a single diffusion step, there are two peaks whose maxima correspond to the step length, $L$, used in the simulation. The value at 0 displacement is non-zero which corresponds to those tracers which have encountered a bead and thus have a final step position equal to the starting step position, which for $j=1$ will be equal to a zero $Z$-displacement. 

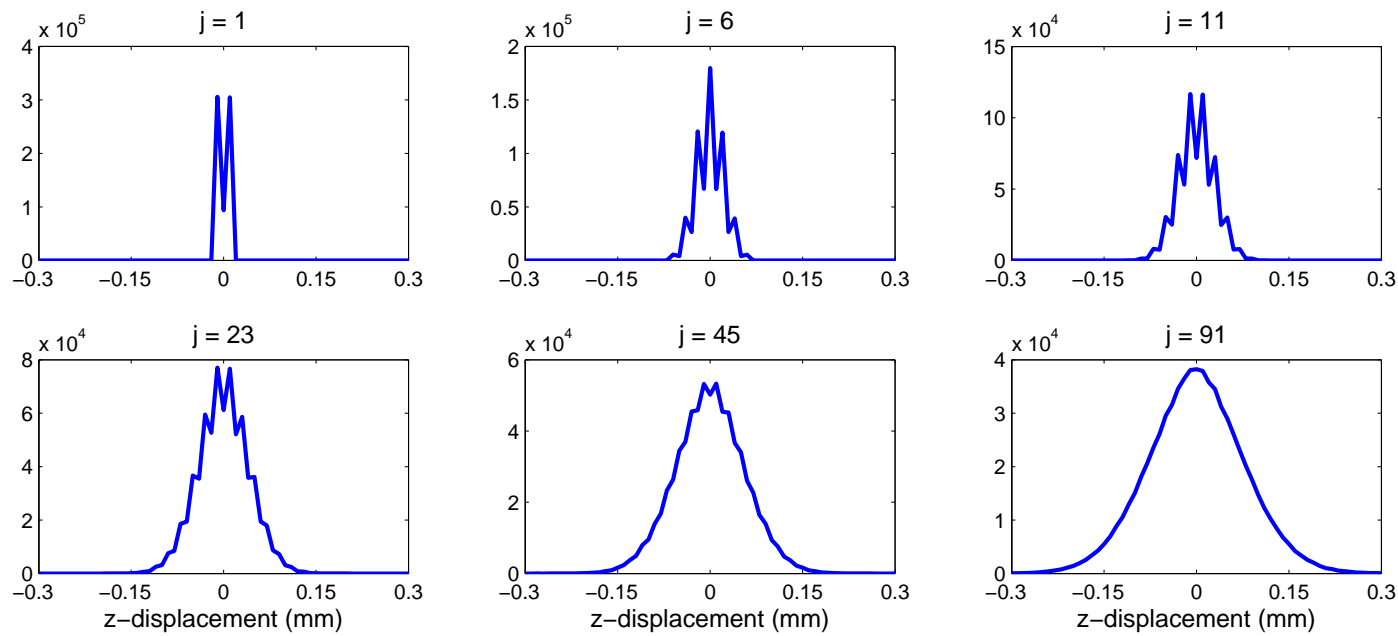

Figure 5.6: The propagators generated for a random walk which is stepped only along the $x y z$ axes. The variable $j$ signifies the number of random walk steps. The discretization of the random walk can be seen at lower $j$ values. At $j=1$ three values of displacement can be seen at $\pm L$ and 0 . The oscillatory nature of the propagators lessens as the number of steps is increased.

As the value of $j$ is increased, the oscillation in the propagators will lessen as the descretization of the $Z$-displacements averages out as more diffusion steps begin to increase the range of maximum $Z$-displacement. It is also possible to reduce the oscillations for the desired diffusion times by reducing $L$ so that a higher number of $j$ will be required for each $\Delta$. However, oscillations will still be present for small values of $j$, and decreasing $L$ will result in a longer computational time.

\subsection{Random Walk with Random Angle}

An alternative to stepping along the $x-, y$ - and $z$ - axes is to step along a random direction, which has the requirement that any single step has an equal chance of falling at any point on the sphere of radius $R_{\text {sphere }}$ centered on $x_{0}, y_{0}$, and $z_{0}$. See the right of figure 5.7, which shows a sample start point and 100 examples of possible steps that could be taken which originate from the start point. The 
green triangle is the start point centered at $(0,0,0)$, and each magenta square represents an end point corresponding to a sample step length of length 1 . The right of figure 5.7 shows the $Z$-displacement for 100,000 tracers with a sample step length $L$ of 1 . Note that the $Z$-displacements span the entire range of $\pm L$.
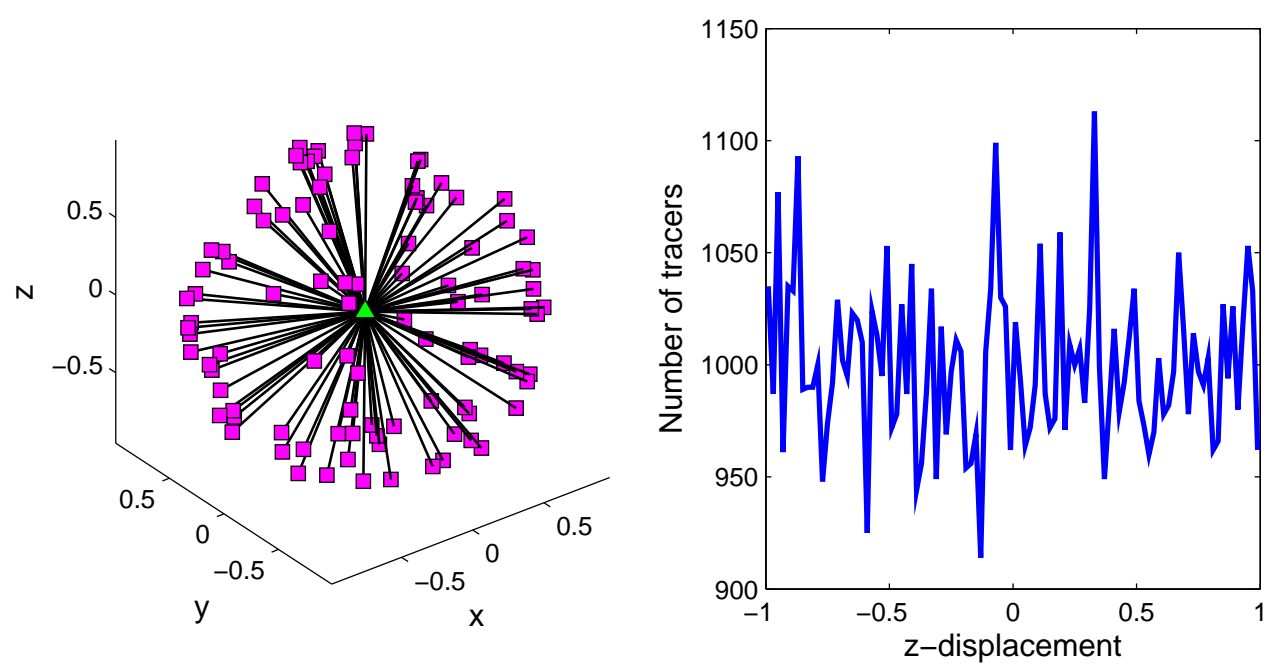

Figure 5.7: Left: An example of 100 random steps a tracer could take starting from point $(0,0,0)$. The green triangle represents the start point, and the magenta squares represent various end points. Right A histogram of the $Z$ displacements of 100,000 tracers, each with a step length of 1 . Note that the $Z$-displacements span the entire range of $\pm L$. The probability is approximated independent of $Z$, as expected.

Rather than defining the step length, $L$, as a fraction of a sphere, it is now dependent on three variables: the diffusion coefficient, $D$, which is $2 \times 10^{-9}$, the total diffusion time, $\Delta$, and the total number of diffusion steps, $N$. $L$ is given by the equation

$$
L=\sqrt{6 D \frac{\Delta}{N}}
$$

and the change in position for each tracer for each diffusion step is given by the 
equations

$$
\begin{aligned}
\Delta x & =L \cos (\phi) \sqrt{1-b^{2}} \\
\Delta y & =L \sin (\phi) \sqrt{1-b^{2}} \\
\Delta z & =L b
\end{aligned}
$$

where $\phi$ is a random angle between 0 and $2 \pi$, corresponding to the azimuthal angle in the $x y$-plane, and $b$ is a random number between -1 and 1 , and represents $\cos (\theta)$, where $\theta$ is the zenith angle.

Allowing the tracer step angle to vary along a sphere will remove the descretization of the $Z$-displacement, since the $Z$-displacement for each step will have the equal probability of falling anywhere between $\pm L$ as shown in the right of figure 5.7, which shows the $Z$-displacement values for the left of figure 5.7. This means that the propagator at $j=1$ will no longer have 3 values but a range of values between $\pm L$. Another major benefit with this procedure is that diffusion time, $\Delta$ is no longer calculated as a number based on $\langle L\rangle$, but is a value hard coded in the simulation to be consistent with a diffusion coefficient of $2 \times 10^{-9} \mathrm{~m}^{2} \mathrm{~s}^{-1}$ making it more comparable with experiment.

Another problem with the original simulation is in the treatment of the tracer reflection off the spheres. Originally, when a tracer encountered a bead, that step was reset to the starting point. It is possible that a tracer can stay at its starting point for a number of steps if it repeatedly encounters a sphere in subsequent steps. To eliminate this possibility as well as make a more realistic simulation we change the procedure so that when a tracer encounters a sphere it will realistically reflect off the sphere surface rather than reflect back to its original position. This reflection needs to be accomplished with knowledge of only the sphere center, the tracer start point, and tracer end point.

As a first step, we reflect off a circle, then expand the method learned here to reflect off a sphere.

\subsubsection{Reflection Off A Circle}

We begin with a circle of radius $R_{\text {circle }}$ and a starting tracer point, $S$, located at $\left(x_{\text {start }}, y_{\text {start }}, z_{\text {start }}\right)$, and generate a test end point, $B$, located at $\left(x_{\text {test }}, y_{\text {test }}, z_{\text {test }}\right)$ 
that is inside the circle centered at $\mathrm{A}\left(x_{0}, y_{0}, z_{0}\right)$. The step angle $\theta$, is a random number between 0 and $2 \pi$, and is the angle of the vector $\overrightarrow{S B}$, where we treat the point $S$ as the origin (see figure 5.8. The length of $\overrightarrow{S B}$ is given by the step length, $L$. To obtain the tracer end point after reflection, $E$, located at $\left(x_{\text {end }}, y_{\text {end }}, z_{\text {end }}\right)$, we need to find the point on the circle where the tracer will reflect off of.

This point will be $C$, located at $\left(x_{C}, y_{C}, z_{C}\right)$.

As $L \ll R_{\text {circle, }}$ we can treat this as a simple reflection problem where the angle of incidence $\theta_{i}$ is equal to the angle of reflection $\theta_{r}$ about a normal line drawn from $A$ through $C$.

$S=$ tracer starting point, $\left(x_{\text {start }}, y_{\text {start }}, z_{\text {start }}\right)$

$A=$ circle center, $\left(x_{0}, y_{0}, z_{0}\right)$

$B=$ tracer end test point, $\left(x_{\text {test }}, y_{\text {test }}, z_{\text {test }}\right)$

$C=$ reflection point, $\left(x_{C}, y_{C}, z_{C}\right)$

$E=$ tracer reflected end point, $\left(x_{\text {end }}, y_{\text {end }}, z_{\text {end }}\right)$

A diagram of the circle is shown on the left side of figure 5.8. The labeling of sides and angles formed by the triangle $A B C$ is shown on the right side of figure 5.8.

We know the location of points $S, A$, and $B$ and want to find point $C$. We can find the length, $c$, of side $A B$ by the norm of vector $\overrightarrow{A B}$. We also know that the length, $b$, of side $A C$ will be $R_{\text {circle }}$ no matter the location of $A$. We can find the cosine of angle $\beta$ between vector $\overrightarrow{A B}$ and vector $\overrightarrow{S B}$ by using the dot product:

$$
\cos \beta=\frac{\overrightarrow{S B} \cdot \overrightarrow{A B}}{|\overrightarrow{S B}||\overrightarrow{A B}|}
$$

Now that we know $\cos (\beta)$, and the lengths $b$ and $c$ we can use the law of cosines to find the length, $a$ of side $B C$. The law of cosines is given by the equation

$$
b^{2}=a^{2}+c^{2}-2 a c \cos (\beta)
$$

Rearranging the above equation gives

$$
0=a^{2}-2 a \cos (\beta)+c^{2}-b^{2}
$$


which is similar to a 2 nd degree polynomial. We can use the quadratic equation to solve for $a$. In this case the correct value of $a$ will be the positive one since a length cannot be negative. From figure 5.8, we can see that $a=|\overrightarrow{B C}|$. Therefore, we can find point $C$ as

$$
\begin{aligned}
& C_{x}=B_{x}+a \cos (\theta-\pi) \\
& C_{y}=B_{y}+a \sin (\theta-\pi) .
\end{aligned}
$$

The normal from point $C$ is just the extension of vector $\overrightarrow{A C}$ outside the sphere. We can see from figure 5.8 that the angle between this extension through point $C$ and vector $\overrightarrow{S C}$ is equal to angle $\gamma$. Therefore the reflection angle between point $S$ and $E$ will be $2 \gamma$. Now that we have the length of a we can again use the law of cosines to find the angle $\gamma$.

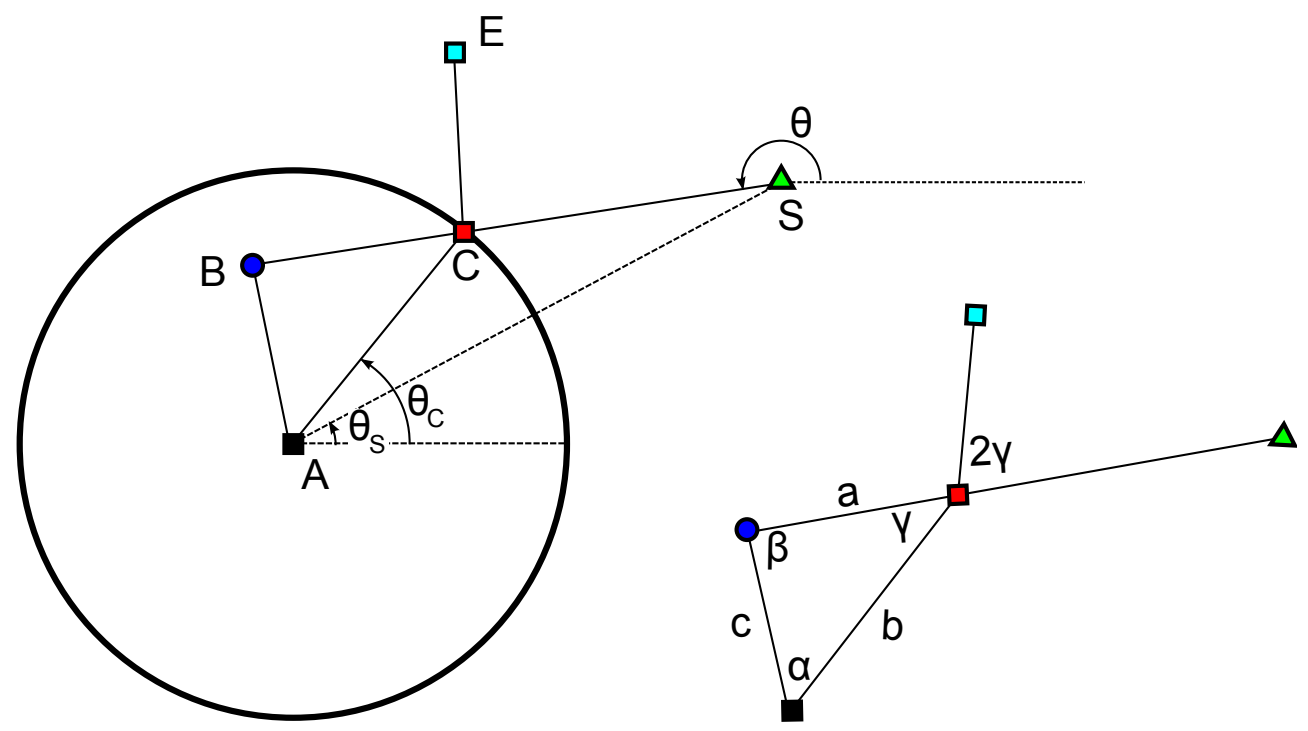

Figure 5.8: Diagram representing the circle centered at $A$ and the tracer with start point $S$, end point $B$, and point $C$ located on the surface of the circle. At right are the same points but with lengths $a, b$, and $c$ and angles $\alpha, \beta$, and $\gamma$ labeled.

$$
\gamma=\cos ^{-1}\left(\frac{a^{2}+b^{2}-c^{2}}{2 a b}\right) .
$$

The reflection angle, in other words, the angle between $\overrightarrow{C S}$ and $\overrightarrow{C E}$, is always $2 \gamma$, however whether the reflection will occur clockwise $\left(\theta_{E}=(\theta-\pi)-2 \gamma\right)$ or 
counter clockwise $\left(\theta_{E}=(\theta-\pi)+2 \gamma\right)$ from the line $\overrightarrow{C S}$, depends on where the reflection point $C$ is relative to the starting point, $S$. In general, we can simply compare the polar angle, $\theta_{C}$ of point $C$ to that of the polar angle, $\theta_{S}$ of point $S$. If $\theta_{C}<\theta_{S}$, then the reflection would occur clockwise, if $\theta_{C}>\theta_{S}$, the reflection would occur counterclockwise. However, there are two special cases where this does not hold.

Case 1: Referring to figure $5.9 \mathrm{a}$, the start point, $S$, shown by the green triangle, occurs in the first quadrant of the circle which corresponds to a $\theta_{S}$ where $0<\theta_{S}<\pi / 2$, and the surface reflection point, $C$, shown by the red square, occurs in the forth quadrant corresponding to a $\theta_{C}$ of $3 \pi / 2<\theta_{C}<2 \pi$. In this case, $\theta_{C}>\theta_{S}$, which will result in the reflection occurring clockwise. A clockwise reflection will cause the final reflection point $E$ to reflect into the circle, as shown by the yellow square. In this case, a counter clockwise reflection is required to obtain the correct final reflection point, shown by the cyan square.

Case 2: The reverse of Case 1. Referring to figure $5.9 \mathrm{~b}$, we have a start point $S$, shown by the green triangle in the forth quadrant corresponding to a $\theta_{S}$ where $3 \pi / 2<\theta_{S}<2 \pi$ and the surface reflection point $C$, shown by the red square occurs in the first quadrant with a $\theta_{C}$ where $0<\theta_{C}<\pi / 2$. Here $\theta_{C}<\theta_{S}$, which will result in a clockwise reflection. A clockwise reflection will result in the final reflection point falling inside the circle as shown by the yellow square. In this case, we need a counterclockwise reflection which will cause the tracer to reflect correctly and result in the final reflection point shown by the cyan square.

We apply a test in the program which checks for these two cases and should either situation arise, both $\theta_{C}$ and $\theta_{S}$ are offset by a value of $2 \pi-\Theta$, where $\Theta=\theta_{C}$ for case 1 , and $\Theta=\theta_{S}$ for case 2 . This offset will correct the inequality comparison between $\theta_{S}$ and $\theta_{C}$ so that $\theta_{E}$ will be calculated properly as shown by the cyan square in both figure $5.9 \mathrm{a}$ and $\mathrm{b}$.

We have the reflection angle, and now we need the length between the reflection point and the end point. This will simply be equal to the length of side $a$. 

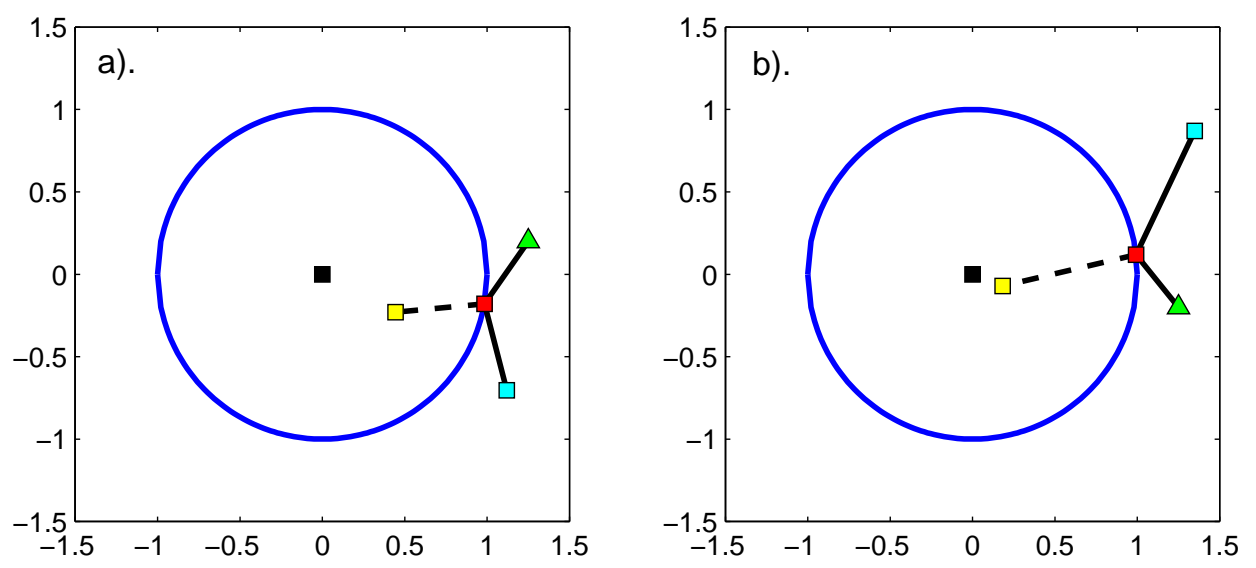

Figure 5.9: a). An example of Case 1 as described above. The start point, shown by the green triangle, falls in the first quadrant, resulting in $0>\theta_{S}>\pi / 2$. The surface reflection point, shown by the red point results in $3 \pi / 2<\theta_{C}<2 \pi$. In this case $\theta_{C}>\theta_{S}$, which normally would result in a clockwise reflection which produces a final reflection point inside the circle as shown by the yellow square. However, our simulation will correct for this situation as described above and will result in the correct final reflection point as shown by the cyan square. b). As for a)., but showing the situation of Case 2 . 
The results of this method are shown in figure 5.10a, where we place a circle of radius $R_{\text {circle }}=1$ around the origin, then generate random start points and step them in such a way that they will encounter the circle and thus bounce back. The tracer start points are shown by the green triangles, the reflection point on the surface of the circle is given by the red squares, and the final reflected tracer point is given by the cyan square. We repeat this in figure $5.10 \mathrm{~b}$ which is the same as a, except the circle has been moved to be centered around $(1,1)$ and has a radius $R_{\text {circle }}=0.5$.
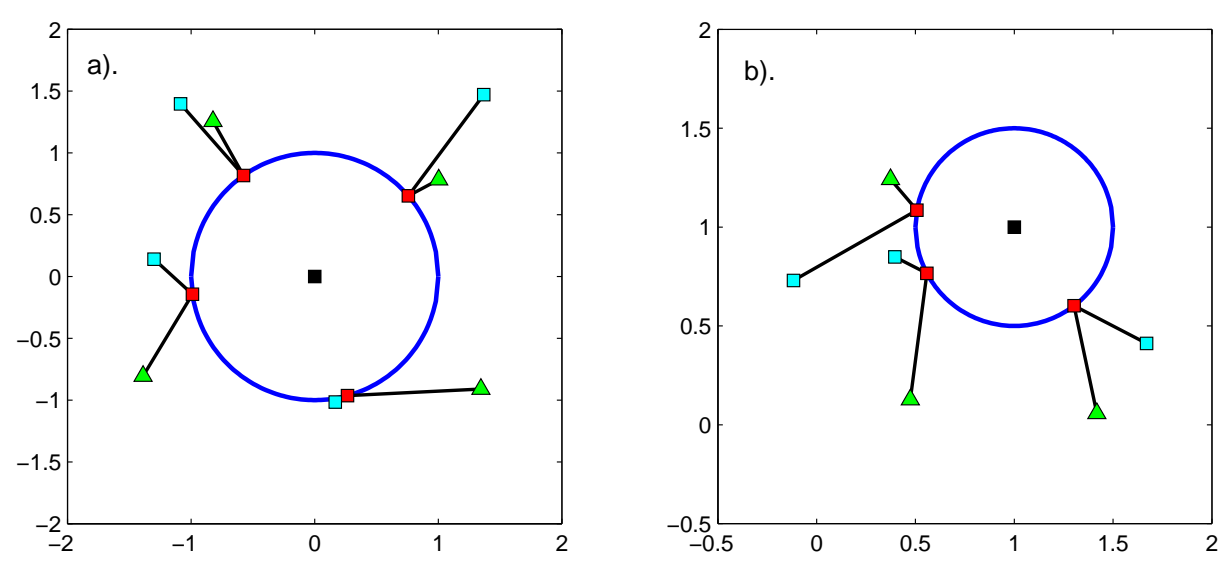

Figure 5.10: Tracer reflection off a circle. a). A circle of radius $R_{\text {circle }}=1$ centered about the origin. Random tracers are generated and stepped into the sphere and bounced off. Tracer start points are given by the green triangle, the point of reflection on the surface of the circle is given by the red square, and the final reflected tracer point is given by the cyan square. b). As for a)., but with a circle of radius $R_{\text {circle }}=0.5$ centered about $(1,1)$.

\subsubsection{Reflection off a Sphere}

Now that we're able to reflect off a circle, we can move on to reflection off a sphere. The points $A, B$ and $S$ all fall on the same plane, which is essentially a map of the circle above, which we will refer to as the "circle plane" from now on. The final point $E$ will also fall on this plane. The first step is to map these 
starting points onto this plane.

Since we have the points $A, B$ and $S$ in Cartesian coordinates, this is easy. We define a point $K$ that will always sit on the circle plane at $x=R_{\text {sphere }}$ and $y=0$ or in polar coordinates, $r=R_{\text {sphere }}$ and $\theta=0^{\circ}$. To find where this point will fall on the sphere we find $\theta_{\text {plane }}$, the angle of the line of intersection between the circle plane and the $x y$-plane by

$$
\theta_{\text {plane }}=\tan ^{-1}\left(\frac{(\vec{n} \times \vec{m})_{\widehat{j}}}{(\vec{n} \times \vec{m})_{\widehat{i}}}\right),
$$

where $\vec{n}$ is the normal vector to the circle plane given by $\vec{n}=\overrightarrow{B A} \times \overrightarrow{S B}$ and $\vec{m}$ is the normal vector to the $x y$-plane, and the subscripts $\widehat{i}$ and $\widehat{j}$ represent the $x$ and $y$ components, respectively. The location of point $K$ on the sphere will be, in cylindrical components, $r=R_{\text {sphere }}, \theta_{K}=\theta_{\text {plane, }}$ and $z=0$. Now that we know the location of point $K$ on the sphere, we can find the values of $\theta_{S K}$ and $\theta_{B K}$ on the circle plane by simply using the dot product as in equation 5.10. The values of $r_{\text {Splane }}$ and $r_{\text {Bplane }}$ are given by $|\overrightarrow{S A}|$ and $|\overrightarrow{B A}|$, respectively. See figure 5.11.

We find point $C_{\text {plane }}$ by using the law of cosines similarly to how we found point $C$ in the original circle in the previous section. After we find $C_{\text {plane }}$ we must map it back to the sphere. Referencing figure 5.12, we know the $C_{x p l a n e}$ and $C_{\text {yplane, }}$ and $\theta_{\text {Cplane }} . r_{C}$ will be $R_{\text {sphere }}$ for both the sphere and circle.

Since we know the angle between the circle plane and the xy-plane, $\phi_{A}$, given by

$$
\phi_{A}=\cos ^{-1}\left(\frac{\vec{n} \cdot \vec{m}}{|\vec{n}||\vec{m}|}\right)
$$

we can find the point $C_{z}$ on the sphere by

$$
C_{z}=C_{\text {yplane }} \sin \left(\phi_{A}\right) .
$$

Points $C_{x}$ and $C_{y}$ are a bit trickier. Refer to figure 5.12 for the variable names in finding points $C_{x}$ and $C_{y}$. First we find the radius of $C$ as a projection onto the xy-plane, and label it $\rho$, which is found by

$$
\rho=\sqrt{R_{\text {sphere }}-C_{z}^{2}}
$$




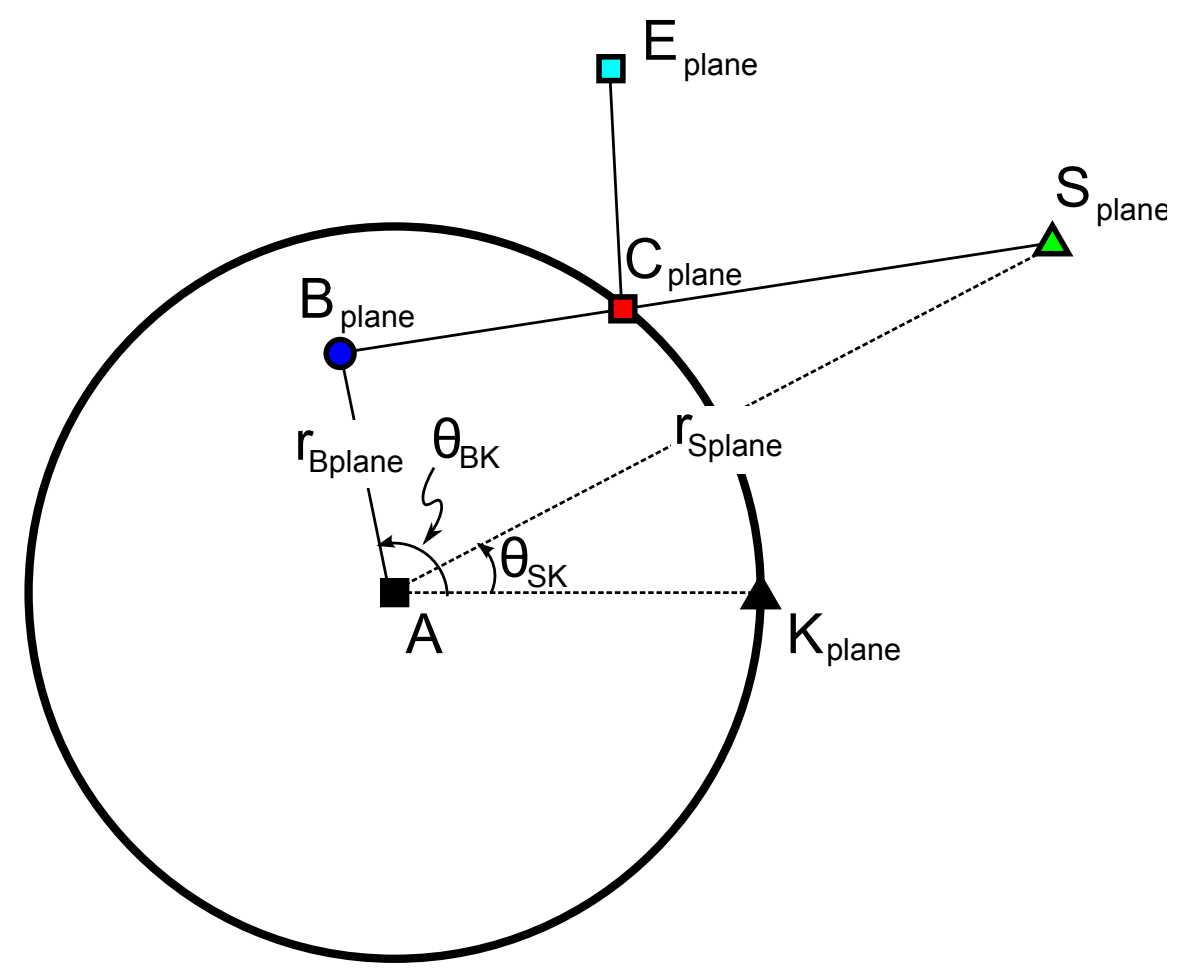

Figure 5.11: A figure of the circle plane showing some of the variables used to calculate $C_{\text {plane. }}$ 


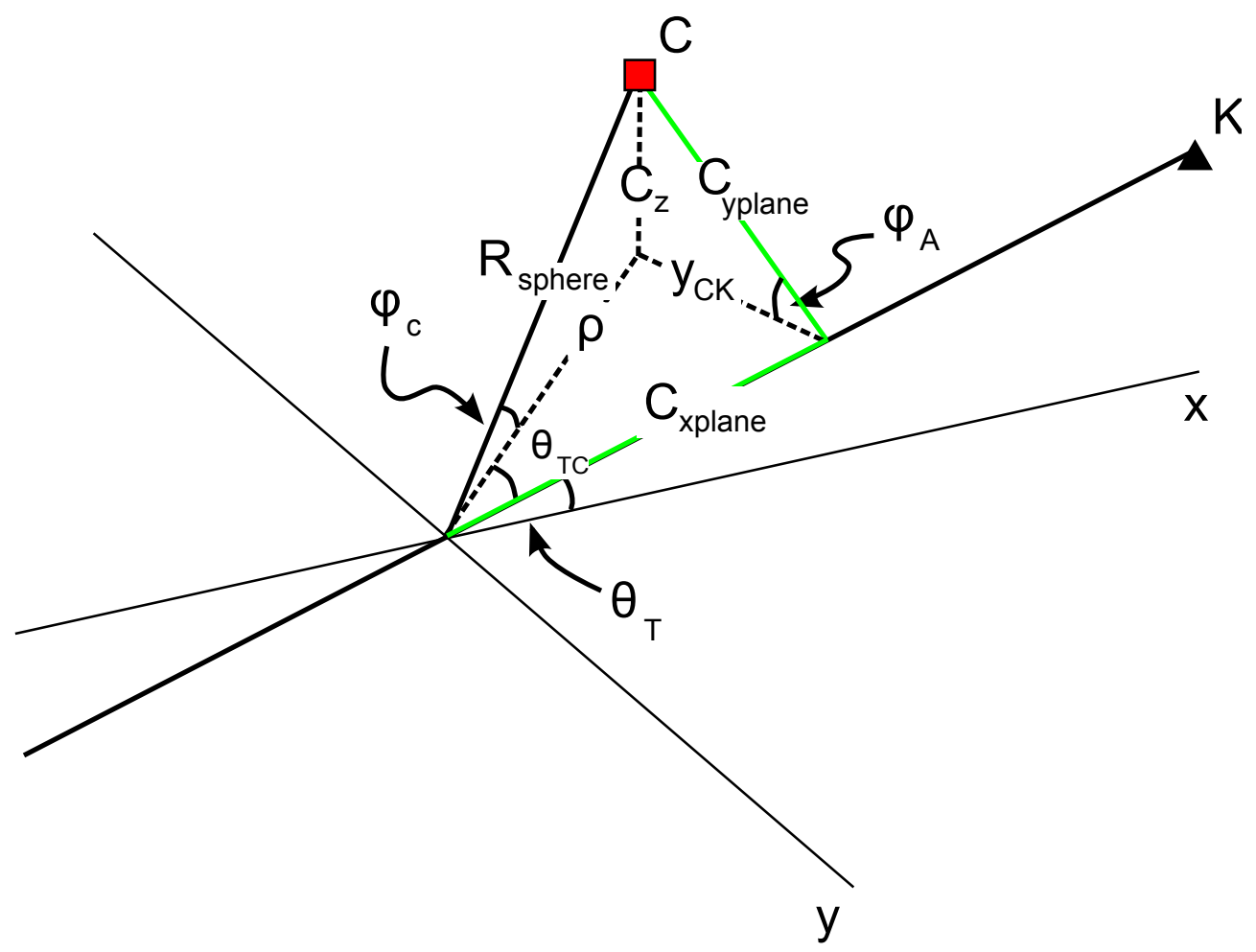

Figure 5.12: A figure of some of the variables used in mapping the surface reflection point $C$ from the circle plane to the sphere. 
The y-value of $C$ on the xy-plane relative to point $K$ is given as $y_{C K}$ and the $\mathrm{x}$-value will be the same as $C_{x p l a n e} . y_{C K}$ is given by

$$
y_{C K}=\sqrt{C_{\text {yplane }}^{2}-C_{z}^{2}} \text {. }
$$

The angle between point $K$ and $C$ on the xy-plane is given by $\theta_{T C}$ and can be found by taking the inverse sine of the ratio of $y_{C K}$ and $\rho$. To find $\theta_{C}$ we simply add the angles $\theta_{K C}$ and $\theta_{T}$ together. The elevation angle to $C, \phi_{C}$ is given by the inverse sine of $C_{z} / R_{\text {sphere }}$. To review, the spherical coordinates of the reflection point $C$ on the sphere are given by

$$
\begin{array}{r}
R_{C}=R_{\text {sphere }} \\
\theta_{C}=\sin ^{-1}\left(\frac{y_{C K}}{\rho}\right) \\
\phi_{C}=\sin ^{-1}\left(\frac{C_{z}}{R_{\text {sphere }}}\right) .
\end{array}
$$

Now that we have the reflection point, $C$, we can find the reflection angle to determine $E$ similarly to how we found point $C$.

First we find $E_{\text {plane }}$ using the same method by which we found the reflection point $E$ on the circle in section 5.6.1. Once we have the Cartesian coordinates for $E_{\text {plane, }}$ we can find the three dimensional coordinates for point $E$ using the same method by which we found the surface reflection point, $C$.

The results of this method are shown in figure 5.13. Figure 5.13a shows a sphere centered about the point $(1,1,1)$ with a radius of 0.5 . The sphere's center is given by the black square. Our tracer start point is given by the green triangle, the original end point inside the sphere is given by the blue circle, the reflection point on the surface of the sphere is given by the red square, and the subsequent reflected end point is given by the cyan square. Point $K$ as described above is given by the black triangle. The circle plane is also plotted in this figure. Note all points fall on this circle plane. b). As for a). but without the circle plane plotted to allow for a better visual of the tracer points. c). The circle plane itself.

To ensure that our points are occurring where they should we have set up a series of error checks in the software. These tests include:

1. Ensuring all points are on the circle plane. 
2. The surface reflection point, $C$, is on the surface of the sphere.

3. The surface reflection point, $C$, is on the line $S B$.

4. The final reflection point, $E$ is not inside the sphere.

5. The angle between $\overrightarrow{C S}$ and $\overrightarrow{C E}$ is $2 \gamma$.

6. The distance between points $C$ and $E$ is equal to the distance between points $C$ and $B$.

Note that to eliminate the chance of a tracer reflecting into another sphere, the sphere radii are reduced from their original radius of $50 \mu \mathrm{m}$ which is used to construct the bead pack, to a radius of $48.6 \mu \mathrm{m}$, ensuring the closest distance between two sphere is $2 L$. While this will slightly increase the porosity of the bead pack and slightly decrease the maximum gradient magnitude, it will not have a large effect on the simulation results.

\subsection{Adding Relaxation}

The total nuclear magnetization of a sample is given by [98]

$$
M(t)=M(0) \sum_{n=0}^{\infty} I_{n} \exp \left(-t / T_{n}\right) .
$$

where $n$ refers to the mode number, and a mode is a single exponential decay. Physically, this equation represents the discretized magnetization decay due to $T_{2}$ relaxation.

For planar geometry

$$
I_{n}=4 \sin ^{2} \xi_{n} / \xi_{n}\left[2 \xi_{n}+\sin \left(2 \xi_{n}\right)\right]
$$

and

$$
T_{n}=a^{2} / D \xi_{n}^{2}
$$

The values of $\xi_{n}$ are given by the positive roots of the transcendental equation

$$
\xi_{n} \tan \left(\xi_{n}\right)=\frac{\rho a}{D},
$$



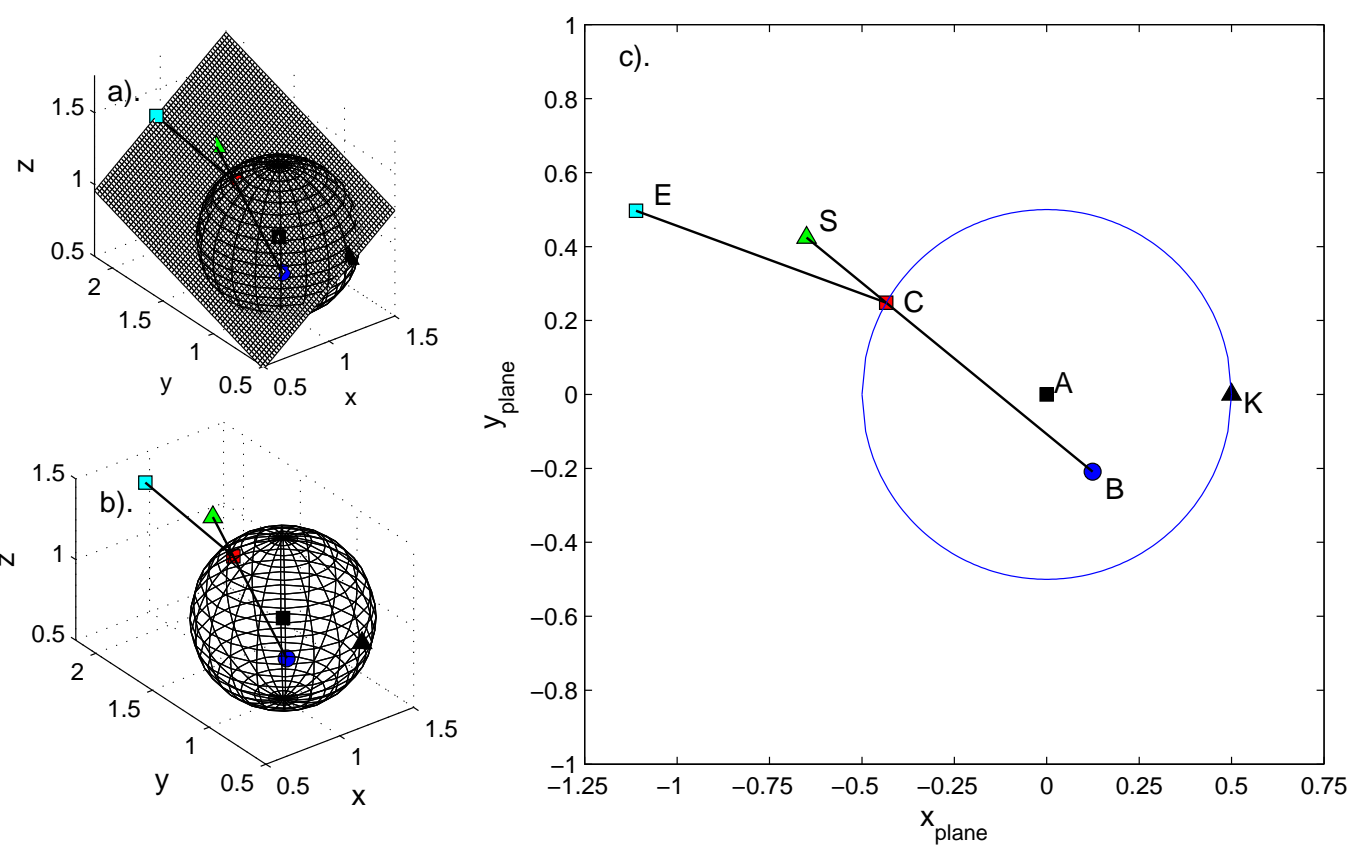

Figure 5.13: a). A test sphere centered about point $A$ shown by the black square at $(1,1,1)$. A start tracer shown by the green triangle has an end point inside the sphere at point $B$, shown by the blue circle. Therefore, the tracer is reflected off the sphere at reflection point $C$ given by the red square and ends up at the final reflection point, $E$, given by the cyan square. Point $K$ is shown by the black triangle. The circle plane is plotted with its relation to the sphere to show that all points lie on the plane. b). As for a). but without the circle plane so as to better show reflection off the sphere. c). The circle plane itself. 
where $a$ is the characteristic length of the system, $D$ is the diffusion coefficient and $\rho$ is a measure of the relaxivity of the system and is always positive. We can use this information to incorporate relaxation into our simulation.

First we need to find the probability that a tracer a distance $L$ from the sphere surface will "relax", i.e. the tracer will be removed from the simulation system. This probability has been calculated in the 3D case by Hunter [99].

A particle a distance $z$ from the sphere surface with a step length $L$ will make a random step given by equations 5.8 and equation 5.9 .

The probability that a tracer particle within a distance $z$ of a wall will step into the wall is given as a fraction of the surface area of the part of the sphere intersecting the wall, $A_{h}$, versus the surface area of the entire sphere, $A_{t}$. $A_{h}$ is

$$
A_{h}=\int_{0}^{\phi} \int_{0}^{2 \pi} L^{2} \sin \phi d \theta d \phi=2 \pi L^{2}(1-\cos \phi)
$$

Given that $\cos \phi=z / L$ and $A_{t}=4 \pi L^{2}$, the probability that a tracer a distance $z$ from the wall will step into the wall is

$$
P_{h}=\frac{1}{2}-\frac{z}{2 L} .
$$

Next we need to define the distribution, $P$ of tracers within a distance $L$ of the wall, the distribution should be uniform so that

$$
P=\frac{1}{L}
$$

To determine the total number of particles within $L$ of the wall to hit the wall, $P_{\text {tot }}$, is

$$
P_{t o t}=\int_{0}^{L} P P_{h} d z=\int_{0}^{L}\left(\frac{1}{2}-\frac{z}{2 L}\right) \frac{1}{L} d z=\frac{1}{4}
$$

We can define the measure of relaxivity in the system, $\rho$, as the rate of particles hitting the wall multiplied by the probability of a particle dying, $k$. We know that the rate of particles hitting the wall is given by $P_{t o t} L / \delta t$, where $\delta t=\Delta / N$. So we can define $\rho$ as

$$
\rho=k \frac{1}{4} \frac{L}{\delta t}
$$

Solving for $k$ :

$$
k=\frac{4 \rho L}{6 D} .
$$


We now have an expression for the probability of a particle dying after hitting a sphere surface, however we still need to determine a value of $\rho$. In 1979, Brownstein and Tarr [98] derived a method to estimate the value of $\rho a / D$ in the slow diffusion regime as

$$
\frac{T_{0}}{T_{i}}=\frac{\alpha \rho a}{D}
$$

where $a$ is the structural characteristic length, which we treat as the distance between tracer barriers, a sphere diameter, $\alpha$ is a constant with a value of 0.41 , and $T_{i}$ and $T_{o}$ are the initial and long time decay times in a decay curve. To find the values of $T_{i}$ and $T_{o}$ to use in our simulation, we fit a double exponential decay curve to a CPMG decay curve for the $100 \mu \mathrm{m}$ diameter bead pack we seek to simulate and find values of $24.5 \mathrm{~ms}$ for $T_{i}$ and $66.9 \mathrm{~ms}$ for $T_{o}$. The CPMG decay and the double exponential fit is shown in figure 5.14. Using these values in equation 5.31 , we determine $k$ to be 0.05 .

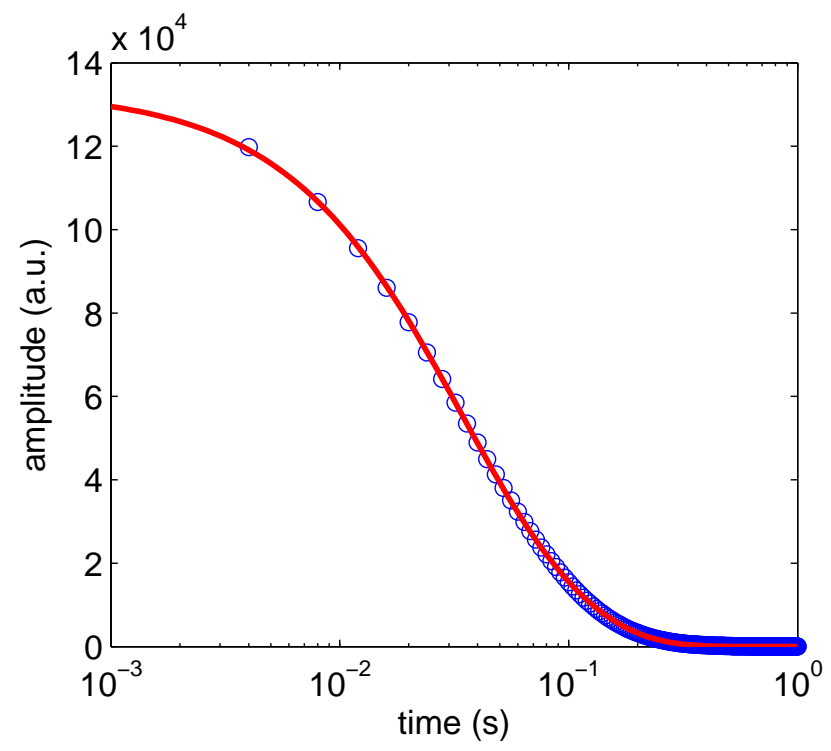

Figure 5.14: A double exponential decay fit to an experimental CPMG experiment of a $100 \mu \mathrm{m}$ monodisperse bead pack used to find $\rho$ in equation 5.31. In this fit we find $T_{i}=24.5 \mathrm{~ms}$ and $T_{o}=66.9 \mathrm{~ms}$.

The value of the probability a tracer will relax when encountering a sphere, $k$, only affects the average position of the $T_{2}$ distribution, and not the width. In figure 5.15, we have run a simulation with approximately 50,000 tracers and 
vary the value of $k$ from 0.05 to 0.95 . Rather than use an inverse Laplace transform on the simulated signal decay, we make a histogram of the number of tracers that have relaxed at specific times in the simulation. From this figure, we can see that increasing the value of $k$ will lead to a decrease in the average $T_{2}$ distribution and a decrease in the distribution's amplitude. The amplitude decrease is due to the rapid loss of tracers at short times as $k$ increases. This rapid loss of tracers at short times as $k$ increases also causes the non-continuity in the $T_{2}$ distribution at short times. This non-continuity is due to the $T_{2}$ times used for data binning being logarithmically spaced from $1 \times 10^{-4}$ to $1 \times 10^{0}$ in 100 bins for the histogram.

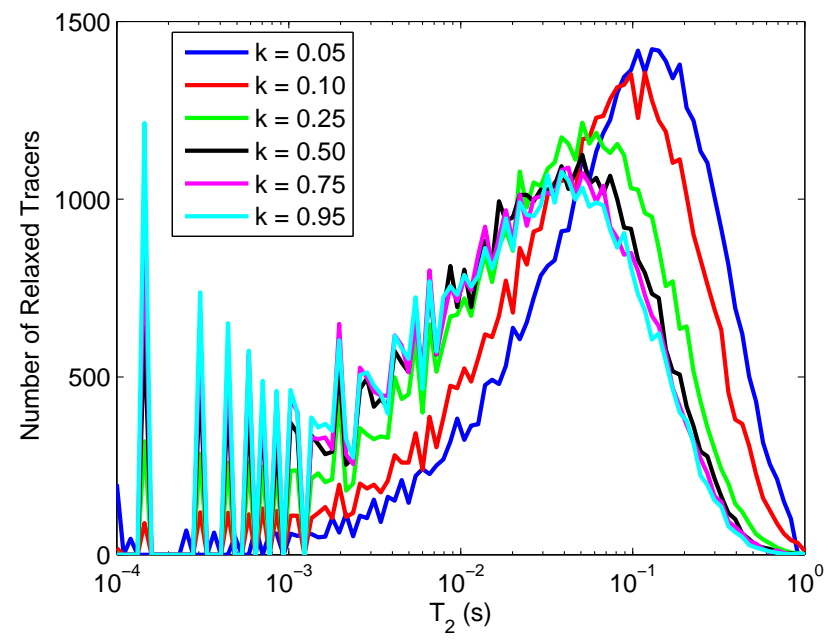

Figure 5.15: $T_{2}$ histograms varying the probability a tracer will relax when encountering a sphere. The number of tracers used is approximately 50,000. $k$ varies from 0.05 to 0.95 .

\subsection{Creating Simulated 2D Spectra}

Given our vectors for the initial internal magnetic field, the internal magnetic field after diffusion, the internal magnetic field gradients, and the relaxation times for each tracer, we can develop 2D spectra to simulate our experimental data. The simulated 2D spectra are simply two-dimensional histograms of 
the desired correlation or exchange spectrum. To simulate our experiments, we have three types of 2D spectra, the first is frequency exchange (chapter 6), given by a histogram of the initial internal magnetic field and and the internal magnetic field after diffusion, secondly $|g|-B_{z}^{i}$ correlation, (chapter 8) which uses the initial internal magnetic field and the internal magnetic field gradient, and finally the $T_{2}-B_{z}^{i}$ correlation (chapter 8 ) which is given by the relaxation times for each tracer and the internal magnetic field after diffusion.

A special case is made for the propagator resolved frequency exchange data (chapter 7). Rather than using solely the simulated initial and post-diffusion internal magnetic field vectors, we incorporate the $Z$-displacement of the tracers, given by $z_{f}-z_{i}$ where $z_{i}$ is the initial $z$ position and $z_{f}$ is the $z$ position after diffusion. Using this $Z$-displacement information, we bin the internal field vectors into a range of $Z$-displacements from 0 to $55 \mu \mathrm{m}$ in $5 \mu \mathrm{m}$ increments. We then proceed to construct the $2 \mathrm{D}$ histograms corresponding to those tracers which have diffused a specific $Z$-displacement.

The 2D spectral results of the simulation will be shown in subsequent experimental chapters.

\subsection{Conclusions}

The development of the simulation used for all subsequent experimental chapters in this thesis was described in this chapter. In it we first show how we generate a randomly packed dense bead matrix, and then show that a simple random walk along the $x y z$-axis is insufficient to portray diffusion through the pore space of this bead pack. This is made evident through the use of simulated propagators, which show oscillatory properties when the diffusion method used is along the $x y z$-axes. We incorporate a diffusion stepping method based on a random walk with the inclusion of more reflection off a bead in cases where a tracer has stepped across the surface of a sphere. Using this diffusion method, we are able to simulate more realistic propagators. We also include a simple method to add $T_{2}$ relaxation by the use of a probability constant that a tracer encountering a sphere will cease to contribute to the simulation - therefore, the tracer 
will have "relaxed". By using the information output from the initial tracer positions and the final tracer positions after the diffusion stepping, we are able to generate multi-dimensional simulated spectra to compare to experiment. 


\section{Chapter 6}

\section{Frequency Exchange}

\subsection{Introduction}

Now that we have introduced the basics of NMR and explained how our simulation works, we now move on to the experimental part of the thesis. The following three chapters will cover the use of multiexponential NMR to try to utilize the inhomogeneous internal magnetic field to characterize the pore space of a sample.

One of the important questions in porous media physics concerns the rate at which imbibed fluid molecules diffuse from pore to pore. In petrophysics, this is particularly relevant as it bears on the question of rock permeability. A number of NMR methods allow for an investigation of fluid diffusion. Pulsed Gradient Spin Echo NMR [24] allows for a direct measurement of molecular translational motion as do spin echo experiments in which one observes signal attenuation due to diffusion in the locally inhomogeneous magnetic field [78]. In 2005 and 2006, a two-dimensional $T_{2}$ exchange method was proposed $[37,39]$ in which one labels molecules in a local pore by virtue of the pore size dependent $T_{2}$ value. Being an exchange method, the technique senses changes in $T_{2}$ values as evidenced by the growth of off-diagonal intensity following a mixing time during which molecules are allowed to diffuse. This method relies on the use of two-dimensional Laplace inversion [100] and is one of a number of 2D separation, correlation and exchange methods [36, 37, 51, 101, 42, 83, 102] 
based on this type of analysis. However there are difficulties associated with the inverse Laplace transform because of pearling effects [103]. This can lead to some ambiguity when it comes to interpreting peak locations. Kuntz et al [40] have proposed a new 2D exchange experiment which involves only the Fourier transformation. This simple exchange method in principle allows one to investigate exchange between pores of imbibed fluid molecules, simply as a result of changes in local Larmor frequencies.

It is well known that porous media in an applied magnetic field will experience internal magnetic field inhomogeneities due to susceptibility differences between the matrix and the pore space. The magnetic field variations will in turn give rise to an inhomogeneous broadening of the NMR lineshape. Such local field inhomogeneity was investigated many years ago by Brown [70] using ferromagnetic grains suspended in water with 5\% carboxy-methylcellulose, and expanded upon by Drain [71] who examined the broadening of magnetic resonance lines in powdered samples. More recently, the internal field has been studied in more depth by Audoly et. al. [74] using a Finney pack of non-penetrating spheres. They find that the variation of the internal magnetic field occurs over the length scale of the pore size in the bead pack, and that its distribution is approximately symmetrical due to the superposition of the field from multiple spheres. Chen et. al. [75] simulate the internal magnetic field for both a fully and partially water saturated Berea sandstone and find similar results to Audoly et. al.

In this chapter, we consider water molecules distributed through the pore space of a porous medium placed in an external magnetic field. As these molecules diffuse, they will experience fluctuations in the local internal magnetic fields arising from inhomogeneities caused by the magnetic susceptibility difference of the matrix and water. For frequency-encoding times that are small compared with the time to diffuse a significant distance in the pore geometry, the 1D spectrum is characteristic of the inhomogeneously broadened line associated with the static distribution of molecule locations. Of course the characteristic time is here associated with the frequency spread associated with the inhomogeneous linewidth, $\tau_{c}^{-1} \sim \delta \omega$. Hence, motional narrowing will occur when the characteristic length, $l$, over which a representative variation of magnetic field is found, 
is such that $l^{2} \delta \omega / D \ll 1$, where $D$ is the self diffusion coefficient of the liquid molecule. In the slow motion limit, $l^{2} \delta \omega / D \gg 1$, frequency encoding of spins gives a good sense of spatial location. By contrast in the fast motion case, spatial localization is lost. This means that the experiment we propose works best for large field inhomogeneity and/or large pores. However, as we shall show here, even when the slow motion condition is relaxed, good estimates of pore-pore exchange times can be made.

The work of Kuntz et al [40] involved a polydisperse glass bead pack in which significant exchange occurred during the frequency encoding time. In this thesis we extend that work to investigate frequency exchange in a monodisperse glass bead pack and using a $B_{0}$ up to $21 \mathrm{~T}(900 \mathrm{MHz})$, thus ensuring the criterion $l^{2} \delta \omega / D \gg 1$.

\subsection{Method}

In an exchange experiment we seek to monitor the migration of molecules to different field positions. This is done by using a pulse sequence similar to a NOESY [35] with two independent frequency-encoding times, $t_{1}$ and $t_{2}$, which lead to frequencies $f_{1}$ and $f_{2}$ which are separated by a mixing time. Ideally the frequency encoding times will be short compared to the mixing period. Figure 6.1 shows the relevant sequence.

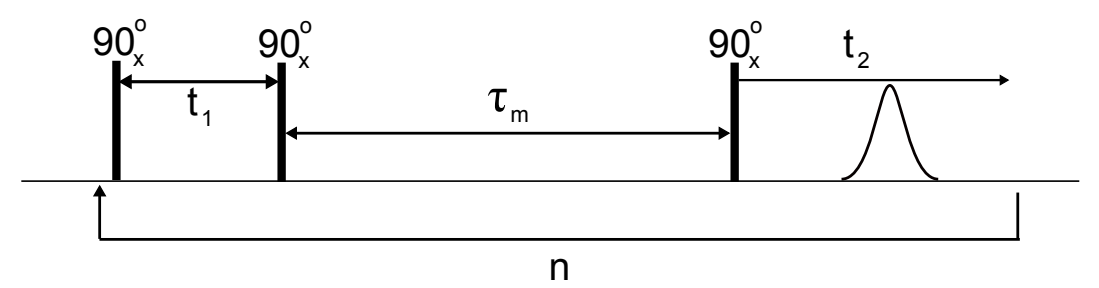

Figure 6.1: 2D Pulse sequence used for local field exchange experiment. The evolution time, $t_{1}$, is increased incrementally for each $\mathrm{n}$. The mixing time, $\tau_{m}$, is kept constant for each repetition, but varies for each experiment.

An initial excitation $90^{\circ}$ pulse rotates the magnetization to the transverse plane. After an evolution time, $t_{1}$, a second storage $90^{\circ}$ pulse brings the mag- 
netization back to the $z$-axis. This inhibits $T_{2}$ relaxation, although the magnetization will still experience $T_{1}$ relaxation. Following this storage pulse, we wait a mixing time $\tau_{m}$, and then apply the third $90^{\circ}$ pulse which rotates the magnetization back to the transverse plane. We then acquire signal over a sampling time labelled $t_{2}$. Phase cycling is used to ensure that the only signal obtained is from that transverse magnetization which derived from the initial $90^{\circ}$ excitation pulse.

This pulse sequence is repeated $n$ times such that for each $n$, the evolution time $t_{1}$ is incrementally increased by an addition of a time equal to 1 /bandwidth. Because of the dephasing that occurs during $t_{1}$, the signal occurs as an echo in the $t_{2}$ domain and with each increment in $t_{1}$, that echo appears at later times with decreasing intensity, a consequence of molecular diffusion, even at the shortest exchange time, $\tau_{m}$. Nonetheless, the echo is always captured in the acquisition window and the attenuation effect leads to some broadening of the spectrum in the $f_{1}$ Fourier domain. However, once the exchange time is increased, the broadening grows significantly as molecules migrate to positions in which the local fields substantially differ. It is the growth of off-diagonal intensity, as a function of mixing time $\tau_{m}$, which we exploit in these measurements

The pulse sequence of figure 6.1 produces an $n \times m$ data matrix where $m$ is given by the number of points acquired during $t_{2}$. From here we apply a $2 \mathrm{D}$ Fourier transform to obtain the 2D frequency spectra shown in the next section. Data was processed using Matlab (The MathWorks, Natick, MA). Samples used were two randomly packed monodisperse glass beads (Duke Scientific Products, Fremont, CA) with diameters of 100 microns and 10 microns. The 100 micron beads were soda lime glass and the 10 micron beads were borosilicate glass.

\subsection{Experimental}

The experiments were performed using Bruker $400 \mathrm{MHz}$ and $900 \mathrm{MHz}$ spectrometers and the proton NMR signal from water imbibed in a randomly packed matrix of monodisperse glass spheres. Specifically, two different samples of 
monodisperse glass beads were used. These had diameters of 100 microns and 10 microns and a standard deviation of $3.6 \mu \mathrm{m}$ and $1.5 \mu \mathrm{m}$, respectively. Different ranges of mixing times were used for each sample depending on bead size. At $400 \mathrm{MHz}$, the bandwidth in both the evolution and detection periods is 20 $\mathrm{kHz}$, with 256 data points being acquired. This leads to a total evolution time less than $13 \mathrm{~ms}$, thus ensuring the diffusive motion of water molecules during the encoding times is much smaller than the bead size in the 100 micron sphere diameter case, and comparable with the sphere size in the 10 micron case. Each experiment took roughly 45 minutes to run with both $n$ and $m=256$.

For comparison, experiments were also performed on a Bruker $900 \mathrm{MHz}$ spectrometer at the University of Queensland in Australia. The higher magnetic field and greater inhomogeneous linewidth allowed us to increase the bandwidth, resulting in a shorter evolution time during frequency encoding. Temperature was held constant at $25^{\circ} \mathrm{C}$ for all experiments.

\subsection{Results and Discussion}

\subsubsection{0 micron Bead Pack}

\section{$400 \mathrm{MHz}$}

The $1 \mathrm{D}$ spectrum of the 100 micron bead pack at $400 \mathrm{MHz}$ is shown in figure 6.2a. Full line width at half maximum (FWHM) is roughly $5.2 \mathrm{kHz}$. Taking the characteristic length to be the bead diameter, $d, d^{2} \delta \omega / D \approx 2 \times 10^{4}$ ensuring that we are in the slow motion limit.

For the 100 micron bead pack, 8 mixing times ranging from 1 to $640 \mathrm{~ms}$ were employed. We were unable to use longer mixing times due to decreased signalto-noise ratio. Figure 6.3 shows the 2D spectra obtained using 256 data points at $20 \mathrm{kHz}$ acquisition and evolution bandwidths. However, to better compare with simulation, we have converted from Hertz to ppm. The longest evolution time is thus $12.8 \mathrm{~ms}$, corresponding to a diffusion length of around 7 microns. Note that each $2 \mathrm{D}$ spectrum has been normalized to constant total intensity so as to remove $T_{1}$ relaxation effects. We note that unlike [40], the $2 \mathrm{D}$ spectra obtained 

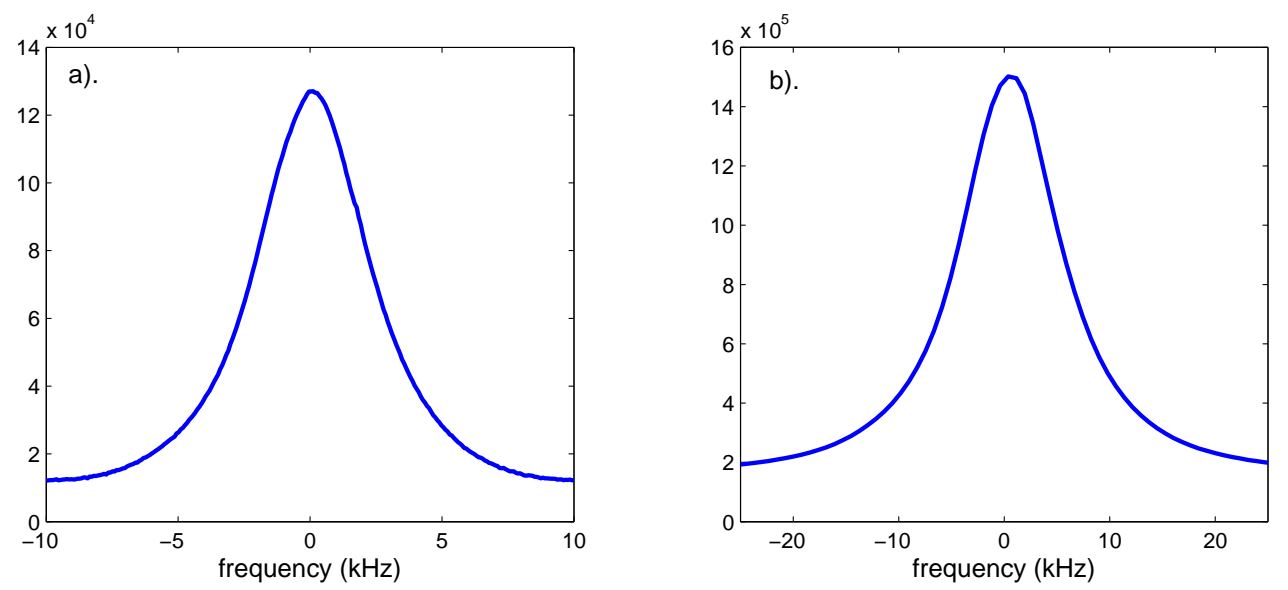

Figure 6.2: a). $1 \mathrm{D}^{1} \mathrm{H}$ NMR spectra obtained from water in 100 micron bead pack at 400 $\mathrm{MHz}$ with a $20 \mathrm{kHz}$ bandwidth. b). as for a). but for $900 \mathrm{MHz}$ and $200 \mathrm{kHz}$ bandwidth.

here are very symmetric.

A clear growth of off-diagonal intensity is observed as the mixing time is increased. This growth is evident as a broadening of the $2 \mathrm{D}$ spectrum in the off-diagonal direction. To quantify this growth, we take an average of two intensities on either side of the off-diagonal corresponding to a total frequency offset equal to a fraction of the FWHM of the 1D spectrum and centered in the diagonal spectrum. Since we normalize the spectra for a constant total intensity, noise can effect the intensity of the center peak. To mitigate this, we take the first row of the $n \times m$ raw data matrix and take the first and last 30 data points which are comprised of only experimental noise. We take an average of these 60 points to get an average noise value, and subtract this value from all data in the $256 \times$ 256 matrix. To ensure that when we select a point on the off-diagonal that isn't within a peak or trough, we take the surrounding 9 points and average them together to get the intensity point. To find the error bars we take the standard deviation and standard error of the first and last 9 points of the first row which will have zero signal.

In figure 6.4 these intensities are plotted against mixing time. We have repeated these measurements of off-diagonal intensity versus mixing time for a range of frequency offsets: 0.6, 0.8, 1.0 and 1.2 FWHM. In each case we fit the 

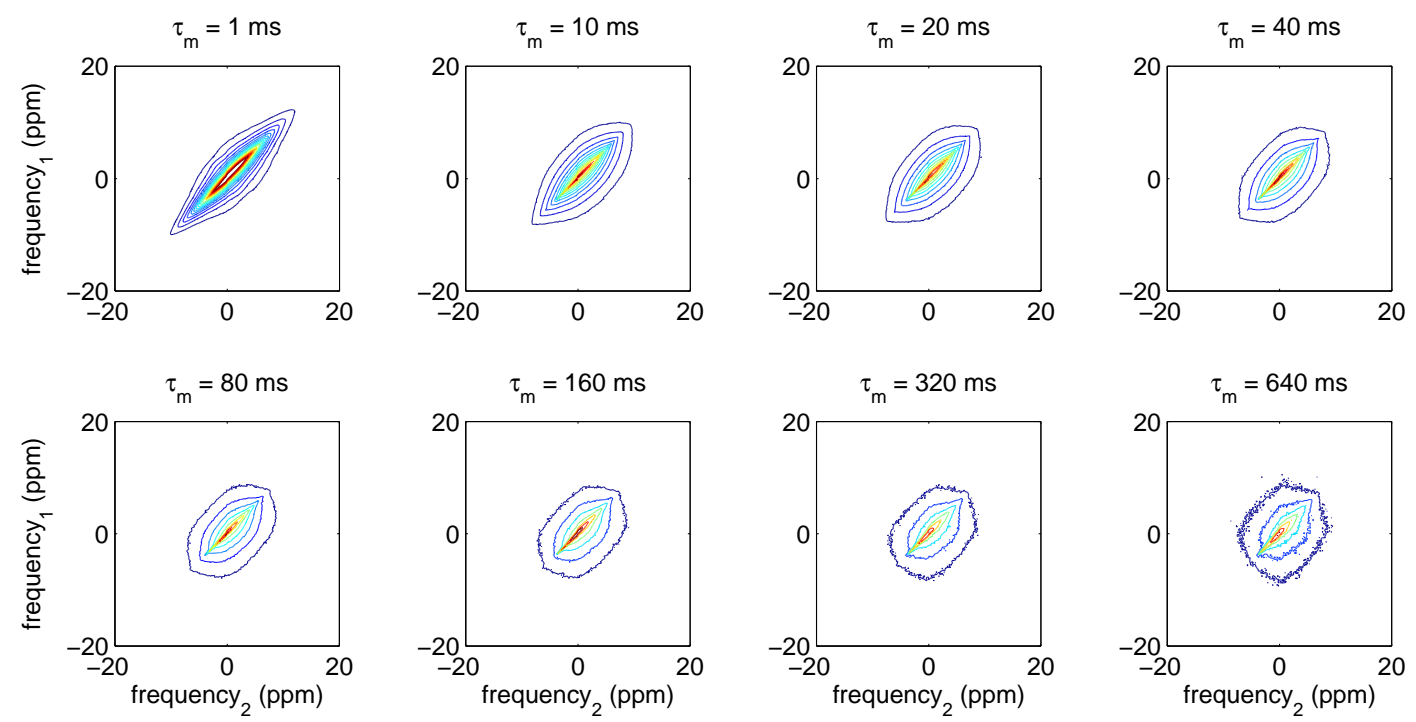

Figure 6.3: $2 \mathrm{D}^{1} \mathrm{H}$ NMR exchange spectra obtained from water in 100 micron bead pack at $400 \mathrm{MHz}$ with a $20 \mathrm{kHz}$ bandwidth. Frequency has been converted from Hertz to ppm to better compare with simulation. The 8 examples show mixing times ranging from 1 to $640 \mathrm{~ms}$. Contours range from $4 \times 10^{-5}$ to $4 \times 10^{-4}$ in $2 \times 10^{-5}$ intervals with blue contours indicating lower intensities and red contours indicating higher intensities. 
data to a bi-exponential growth relation $a_{0}+a_{1} \exp \left(\frac{-t}{\tau_{1}}\right)+a_{2} \exp \left(\frac{-t}{\tau_{2}}\right)$. While the precision of the data justify a fit to more than one exponential, we acknowledge that a bi-exponential model may be simplistic and that a continuum of characteristic times may exist. Table 6.1 shows the values of the parameters obtained. Note that we have large values of $a_{0}$ due to diffusion during our encoding time contributing to off-diagonal broadening. However, there is a remarkable consistency of behaviors for whatever frequency offset is chosen for the analysis. In particular, we find characteristic times of $\tau_{1} \approx 6-11 \mathrm{~ms}$ and $\tau_{2} \approx 250-900$ $\mathrm{ms}$, the short time component $\left(a_{1}\right)$ dominates at smaller offsets and the long time constant component $\left(a_{2}\right)$ dominates at large offsets. Note that the $\tau_{1}$ and $\tau_{2}$ time constants correspond roughly with $1 \mathrm{D}$ diffusive distances of 0.05 - 0.06 and $0.32-0.60$ bead diameters respectively given a bulk diffusion constant of $D_{0}=2 \times 10^{-9} \mathrm{~m}^{2} \mathrm{~s}^{-1}$.
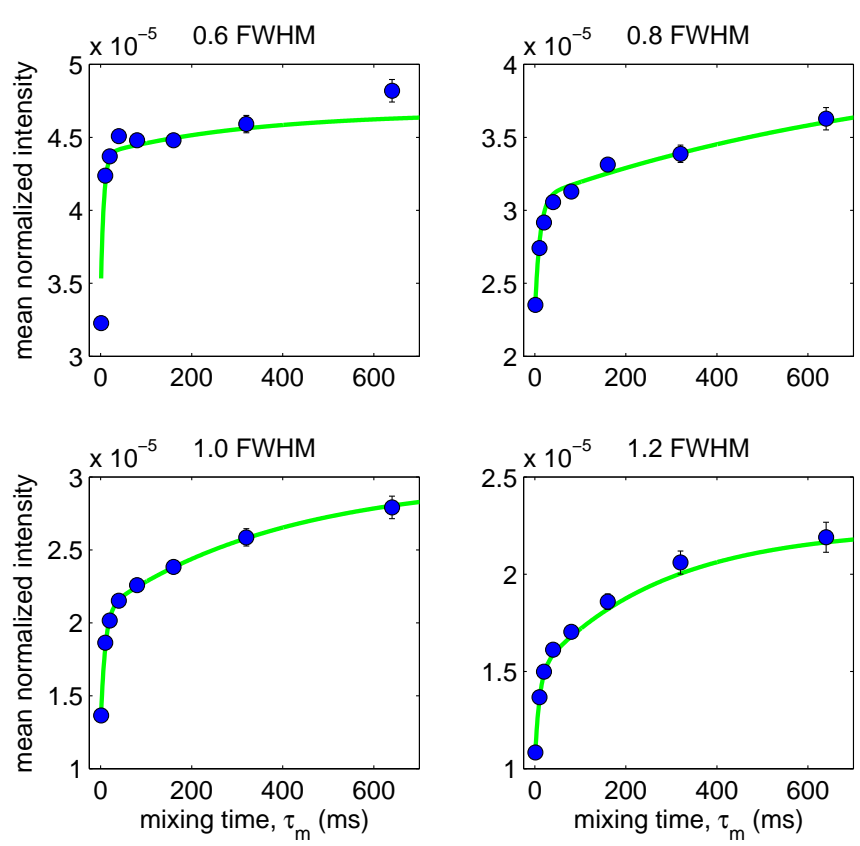

Figure 6.4: Mean normalized intensities located at equidistant points of the center peak along the off-diagonal of spectra shown in figure 6.3 with respect to mixing time. The solid green line is a fit to a bi-exponential growth $a_{0}+a_{1} \exp \left(\frac{-t}{\tau_{1}}\right)+$ $a_{2} \exp \left(\frac{-t}{\tau_{2}}\right)$. 


\begin{tabular}{|c||c|c|c|c|}
\hline & 0.6 FWHM & 0.8 FWHM & 1.0 FWHM & 1.2 FWHM \\
\hline \hline$a_{0}$ & $4.7 \times 10^{-5}$ & $4.1 \times 10^{-5}$ & $3.0 \times 10^{-5}$ & $2.2 \times 10^{-5}$ \\
\hline$a_{1}$ & $-9.9 \times 10^{-6}$ & $-8.0 \times 10^{-6}$ & $-8.0 \times 10^{-6}$ & $-4.6 \times 10^{-6}$ \\
\hline$a_{2}$ & $-2.8 \times 10^{-6}$ & $-9.9 \times 10^{-6}$ & $-9.0 \times 10^{-6}$ & $-7.4 \times 10^{-6}$ \\
\hline$\tau_{1}$ & $6.7 \mathrm{~ms}$ & $11.0 \mathrm{~ms}$ & $9.7 \mathrm{~ms}$ & $9.8 \mathrm{~ms}$ \\
\hline$\tau_{2}$ & $333 \mathrm{~ms}$ & $870 \mathrm{~ms}$ & $402 \mathrm{~ms}$ & $278 \mathrm{~ms}$ \\
\hline
\end{tabular}

Table 6.1: Parameters obtained for figure 6.4 with the bi-exponential growth relation $a_{0}+a_{1} \exp \left(\frac{-t}{\tau_{1}}\right)+a_{2} \exp \left(\frac{-t}{\tau_{2}}\right)$.

\section{$900 \mathrm{MHz}$}

As we stated in section 6.2, we want to minimize the diffusion that occurs during the evolution time. By increasing the magnitude of the external magnetic field, we can increase the internal magnetic field inhomogeneity and thus increase the broadening of the spectrum. This broadening will allow us to increase the spectral width which in turn will reduce the size of the $t_{1}$ increments. For the $900 \mathrm{MHz}$ experiments, evolution and acquisition bandwidth was increased by an order of magnitude to $200 \mathrm{kHz}$ while $n$ was increased to 512 for a maximum $t_{1}$ of less than $3 \mathrm{~ms}$, helping minimize the maximum diffusion distance during the evolution time to around 3 microns. Mixing times cover a range from 1 to $640 \mathrm{~ms}$. Figure $6.2 \mathrm{~b}$ shows the 1D spectrum and figure 6.5 shows the 2D spectra obtained. Even though the bandwidth is $200 \mathrm{kHz}$, these figures show a bandwidth of $40 \mathrm{kHz}$ to better display the spectra. The FWHM 1D spectrum is approximately $11.8 \mathrm{kHz}$, which is roughly $9 / 4$ broader than the $1 \mathrm{D}$ spectrum at $400 \mathrm{MHz}$, yielding $d^{2} \delta \omega / D \approx 5 \times 10^{4}$. All 2D spectra are normalized with respect to total intensity.

One of the most noticeable qualities of the $900 \mathrm{MHz}$ data is the improved signal-to-noise ratio at longer mixing times. However, we do see a vertical spread of very low intensities in the $f_{1}$ dimension likely due to phase cycling errors. As with the $400 \mathrm{MHz}$ data, we see a growth of off-diagonal intensity as mixing time is increased indicated by a line broadening in the off-diagonal direction. We quantify this broadening by taking the mean intensity of two points 

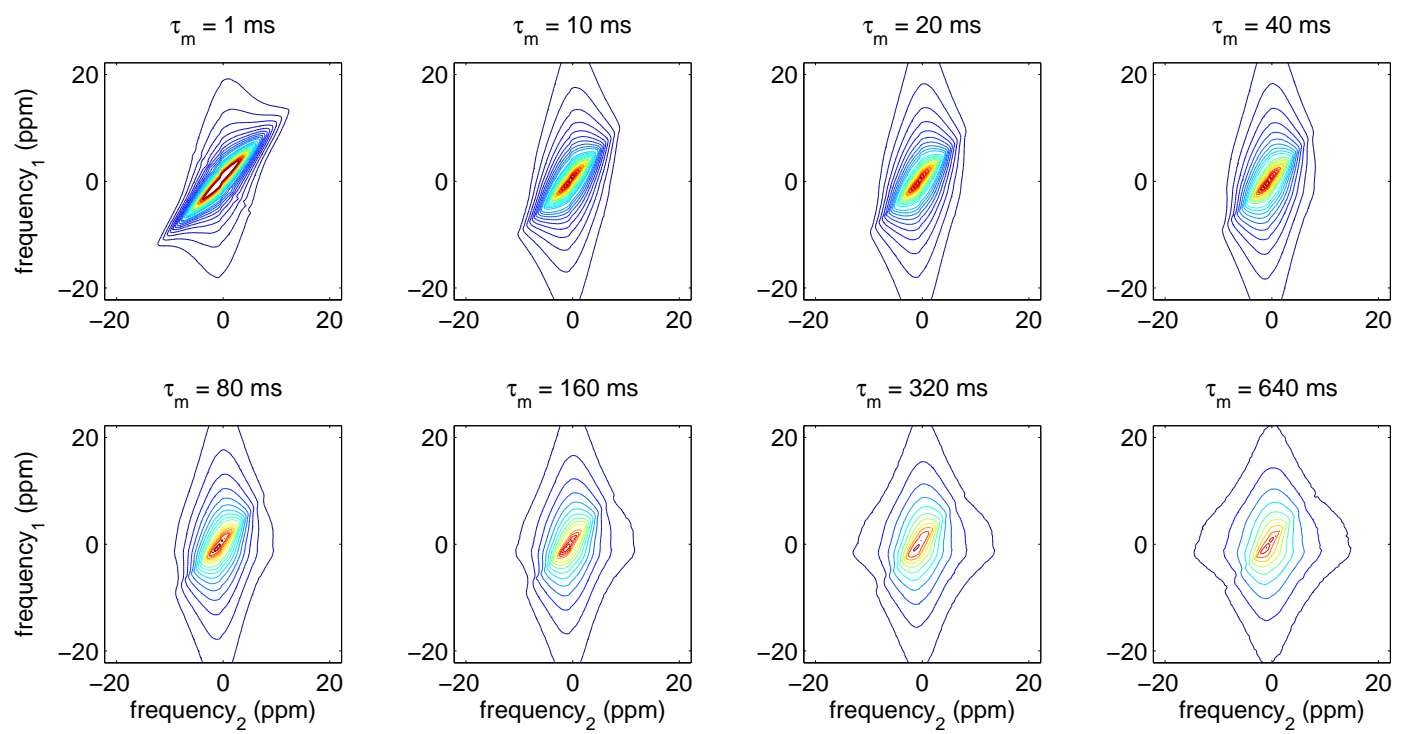

Figure 6.5: $2 \mathrm{D}{ }^{1} \mathrm{H}$ NMR exchange spectra obtained from water in 100 micron bead pack at $900 \mathrm{MHz}$ with a $200 \mathrm{kHz}$ bandwidth. The bandwidth shown is $40 \mathrm{kHz}$. The 8 examples show mixing times ranging from 1 to $640 \mathrm{~ms}$. Intensities range from $1 \times 10^{-4}$ to $2 \times 10^{-3}$ with intervals of $1 \times 10^{-4}$. Blue contour lines indicate lower intensities, with red contour lines indicating higher intensities.

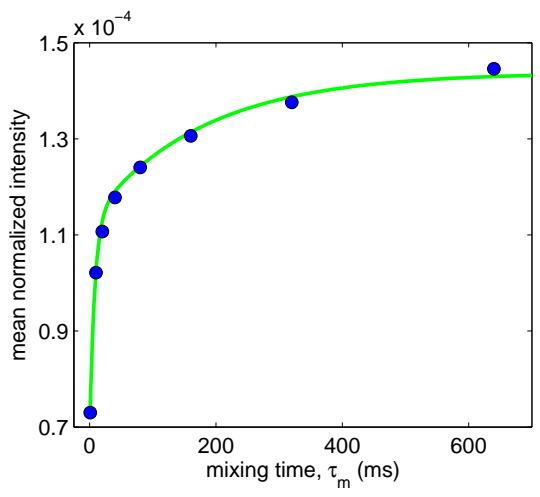

Figure 6.6: Mean normalized intensities located at equidistant points of the center peak along the off-diagonal of spectra shown in figure 6.5 with respect to mixing time. The solid green line is a fit to bi-exponential growth $a_{0}+a_{1} \exp \left(\frac{-t}{\tau_{1}}\right)+$ $a_{2} \exp \left(\frac{-t}{\tau_{2}}\right)$. The error bars in this case are smaller than the data markers. 
equidistant from the center peak, again at a frequency offset equal to the inhomogeneous linewidth. These mean intensities are shown in figure 6.6 along with a fit to the biexponential growth model.

The biexponential growth observed here is similar to that obtained from the $400 \mathrm{MHz}$ experiments. Mean intensities are greater for the $900 \mathrm{MHz}$ results than the $400 \mathrm{MHz}$ results due to the higher signal to noise ratios at this magnetic field. Characteristic bi-exponential growth times are again on the order of $7.85 \mathrm{~ms}$ and $177 \mathrm{~ms}$. These correspond to water molecule rms diffusion distances of 0.06 and 0.27 bead diameters respectively.

\subsection{Simulation}

The simulation we use is described in depth in the previous chapter. We take approximately 1.2 million tracers in the pore space of our sample cylinder for a tracer density of about 7 tracers per $10 \mu \mathrm{m}^{3}$. After the internal magnetic field is calculated at the tracer starting positions, the tracers diffuse randomly through the pore space for a maximum simulation time of $640 \mathrm{~ms}$ to replicate the experiment. The final tracer positions are recorded at simulation times equivalent to the experimental times. Using the final tracer positions, the final internal magnetic field for each mixing time is calculated and by use of both the final and initial fields a two-dimensional simulated spectrum for each mixing time can be created as shown in figure 6.7. Since the simulated frequency is dimensionless, it is converted to ppm to better compare to experiment.

As in the experiment, the simulated data shows an increase in off-diagonal intensity with increasing mixing time. The simulated off-diagonal intensity growth is much more obvious compared to that in figure 6.3, due to no experimental noise, no influence of $T_{1}$ relaxation and no diffusion during the encoding time as the simulation only diffuses tracers for a time equivalent to $\tau_{m}$. As before, to quantify the off-diagonal intensity growth we take the mean intensity of two points which are equidistant from the main center point. The same FWHMs as the $400 \mathrm{MHz}$ experiment using $100 \mu \mathrm{m}$ beads are used and are shown in figure 6.8. The mean intensities at each mixing time are shown by the blue circles 

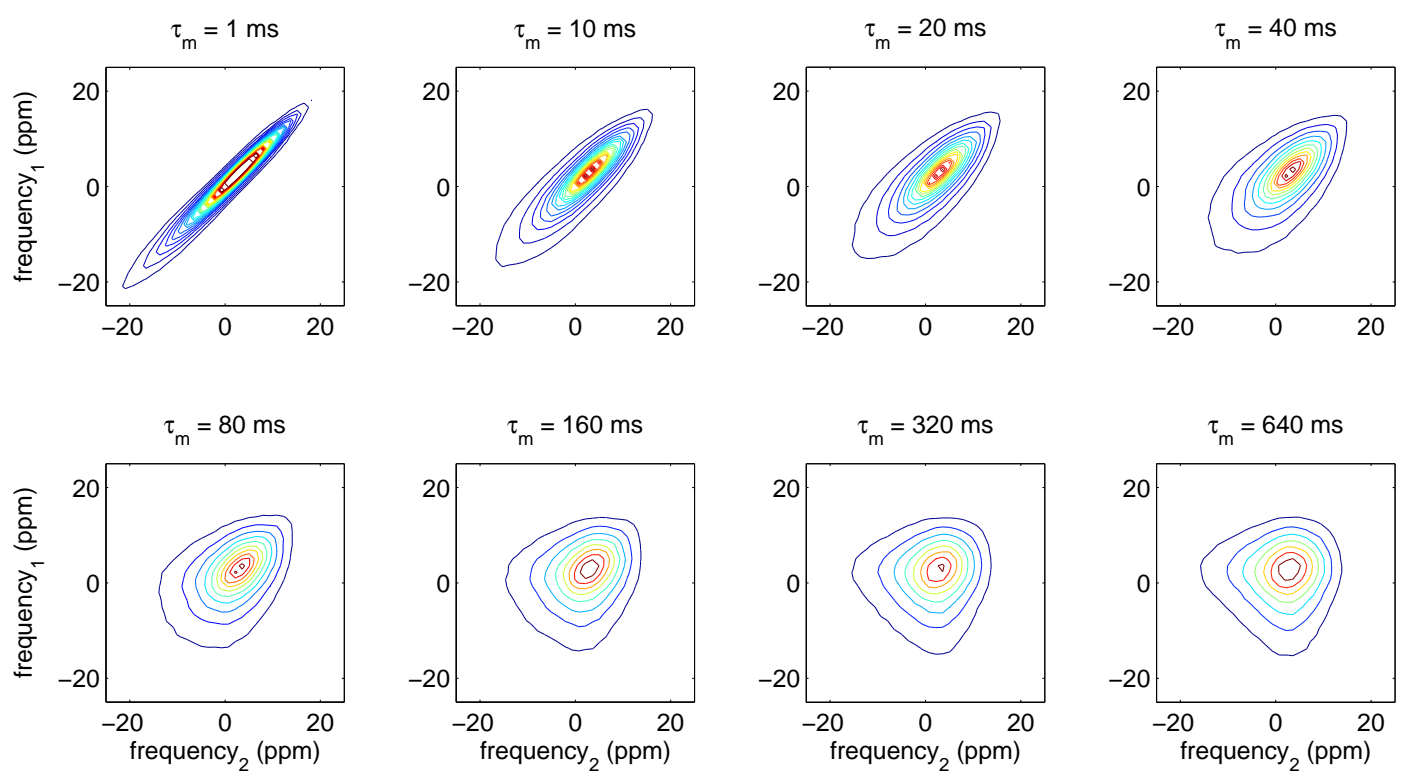

Figure 6.7: Simulated exchange spectra for a $100 \mu \mathrm{m}$ bead pack with mixing times ranging from 1 to $640 \mathrm{~ms}$. Intensities range from $1 \times 10^{-3}$ to $2.5 \times 10^{-2}$ with intervals of $9.6 \times 10^{-4}$. Blue contour lines indicate lower intensities, with red contour lines indicating higher intensities. 
and are fit to a biexponential. The resultant fits are shown by the solid green lines and the values for these fits are shown in table 6.2. Similar to experiment, simulated characteristic times range from 9 - $15 \mathrm{~ms}$ and $100-250 \mathrm{~ms}$ which correspond to $0.06-0.08$ bead diameters and $0.21-0.32$ bead diameters, respectively.
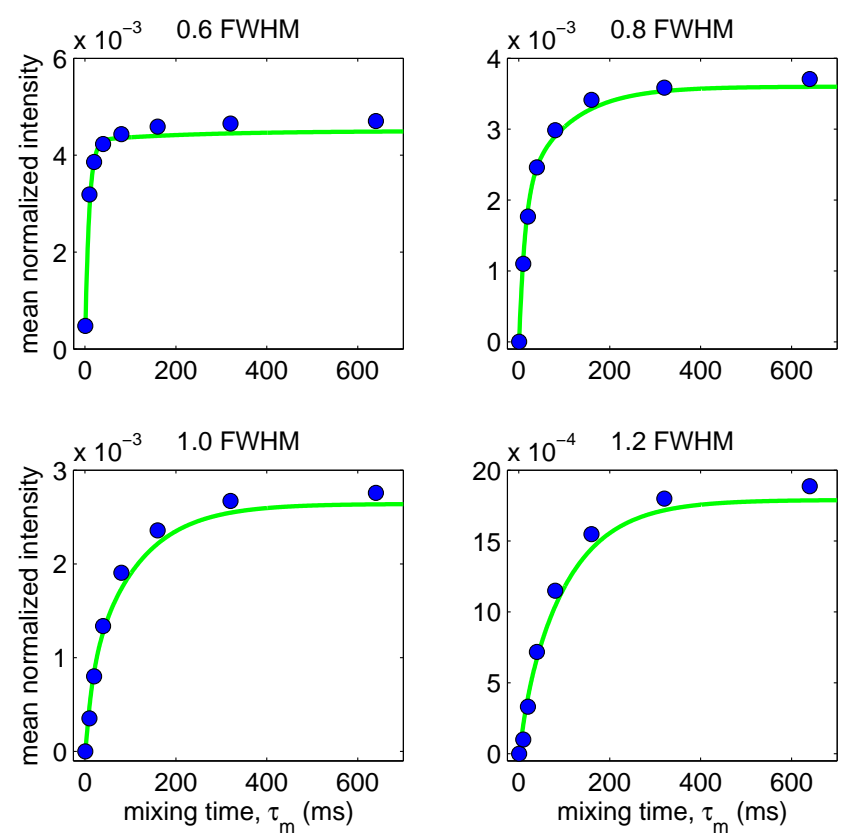

Figure 6.8: Mean normalized intensities located at equidistant points of the center peak along the off-diagonal of spectra shown in figure 6.7 with respect to mixing time. The solid green line is a fit to a bi-exponential growth $a_{0}+a_{1} \exp \left(\frac{-t}{\tau_{1}}\right)+$ $a_{2} \exp \left(\frac{-t}{\tau_{2}}\right)$.

\subsubsection{0 micron Bead Pack}

For porous media for which the slow limit, $l^{2} \delta \omega / D \gg 1$, does not apply, frequency exchange during the encoding periods $t_{1}$ and $t_{2}$ will lead to severe broadening of the 2D spectra even at the shortest mixing times employed. Nonetheless, the effect of an increasing mixing time may be sufficient to slightly enhance this broadening in the case where extreme motional averaging does not apply. For that reason we have attempted to carry out our 2D exchange experiments 


\begin{tabular}{|l||c|c|c|c|}
\hline & 0.6 FWHM & 0.8 FWHM & 1.0 FWHM & 1.2 FWHM \\
\hline \hline$a_{0}$ & $4.5 \times 10^{-3}$ & $3.6 \times 10^{-3}$ & $2.3 \times 10^{-3}$ & $1.8 \times 10^{-3}$ \\
\hline$a_{1}$ & $-4.4 \mathrm{e}-5 \times 10^{-3}$ & $-2.2 \times 10^{-3}$ & $-8.2 \times 10^{-4}$ & $-2.1 \times 10^{-4}$ \\
\hline$a_{2}$ & $-2.0 \times 10^{-4}$ & $-1.6 \times 10^{-3}$ & $-1.9 \times 10^{-3}$ & $-1.6 \times 10^{-3}$ \\
\hline$\tau_{1}$ & $8.8 \mathrm{~ms}$ & $13.0 \mathrm{~ms}$ & $14.7 \mathrm{~ms}$ & $14.5 \mathrm{~ms}$ \\
\hline$\tau_{2}$ & $233 \mathrm{~ms}$ & $100 \mathrm{~ms}$ & $106 \mathrm{~ms}$ & $103 \mathrm{~ms}$ \\
\hline
\end{tabular}

Table 6.2: Parameters obtained for figure 6.8 with the bi-exponential growth relation $a_{0}+a_{1} \exp \left(\frac{-t}{\tau_{1}}\right)+a_{2} \exp \left(\frac{-t}{\tau_{2}}\right)$.

for beads of 10 micron diameter. The diffusion distances at longest evolution time, 7 microns and 3 microns, for 400 and $900 \mathrm{MHz}$, correspond to a significant fraction of one bead diameter.

\section{$400 \mathrm{MHz}$}

The 1D spectrum for the 10 micron bead pack at $400 \mathrm{MHz}$ gives a half height width of $2.9 \mathrm{kHz}$, smaller than that seen for the 100 micron beads presumably due to a different diamagnetic susceptibility for this particular glass. Here $d^{2} \delta \omega / D \approx 10^{2}$.

For the 10 micron bead pack, 12 mixing times are employed ranging from 1 to $40 \mathrm{~ms}$. The smaller range of mixing times is needed because it takes less time for a water molecule to diffuse through the smaller pore space in the 10 micron bead pack compared to the larger pore space of the 100 micron bead pack. The resultant spectra can be seen in figure 6.9. These $2 \mathrm{D}$ spectra have also been normalized to constant total intensity in order to remove $T_{2}$ relaxation effects. Because of the rapid motional averaging occurring during the frequency encoding times, the 2D spectra are very broad, even at the shortest mixing time. Consequently, the growth of off-diagonal spectral characteristics is not as noticeable as found for the 100 micron beads. The shortest mixing time lacks a sharply diagonal spectrum. Even during the $1 \mathrm{~ms}$ mixing time, significant diffusion has taken place compared to that for the $100 \mu \mathrm{m}$ sample. Nonetheless, some additional broadening is visible over mixing times from 1 to $20 \mathrm{~ms}$, just enough for 

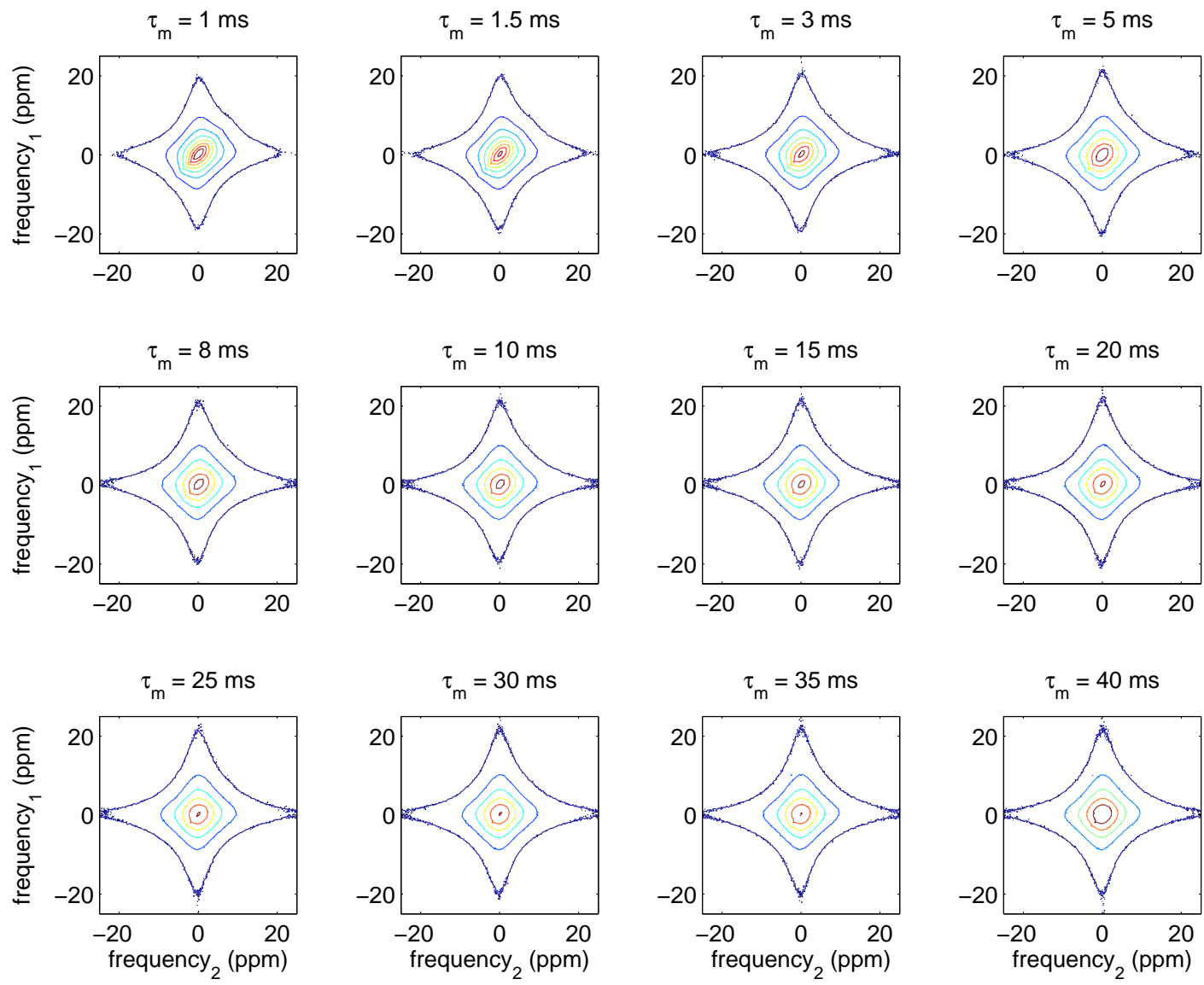

Figure 6.9: $2 \mathrm{D}{ }^{1} \mathrm{H}$ NMR exchange spectra obtained from water in 10 micron bead pack at $400 \mathrm{MHz}$ with a bandwidth of $20 \mathrm{kHz}$. The 12 examples show mixing times ranging from 1 to $40 \mathrm{~ms}$. Intensities range from $2 \times 10^{-5}$ to $1.6 \times 10^{-4}$ with intervals of $1.75 \times 10^{-5}$. Blue contour lines indicate lower intensities, with red contour lines indicating higher intensities. 
us to be able to observe pore exchange effects. This residual visibility results from the fact that it takes about $25 \mathrm{~ms}$ for a water molecule to diffuse one bead diameter. The line broadening can again be quantified by taking the average of two intensities at equidistant points from the center peak and plotting them against mixing time, shown in figure 6.10. Again we see a growth, albeit over a shorter time scale than that of the 100 micron bead pack.

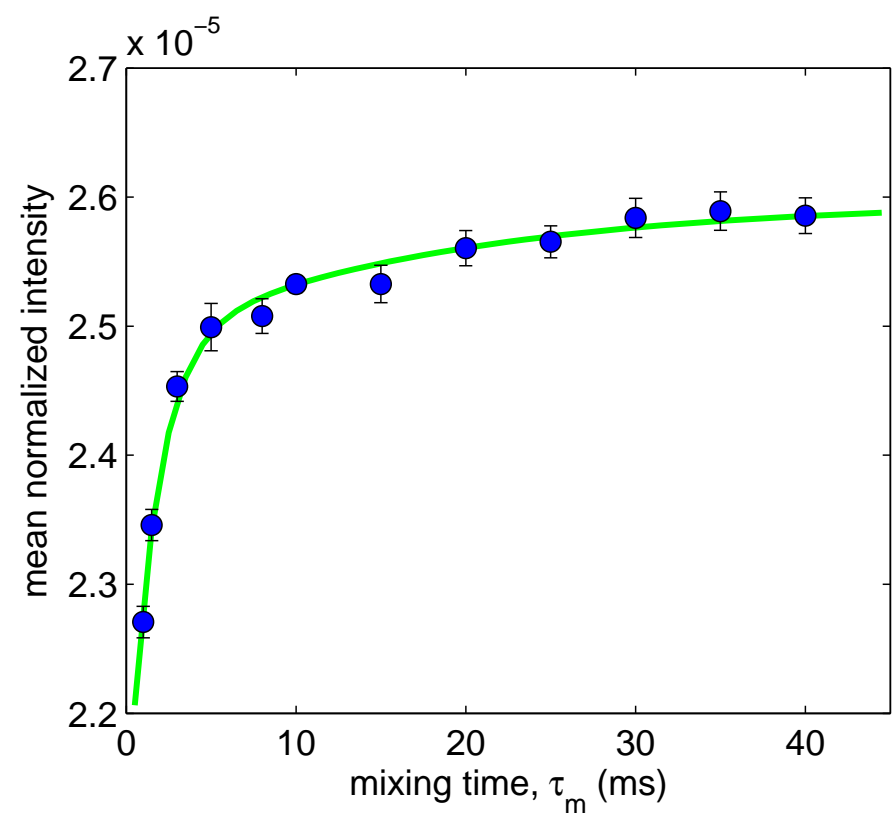

Figure 6.10: Mean normalized intensities located at equidistant points of the center peak along the off-diagonal of $400 \mathrm{MHz}$ spectra shown in figure 6.9 with respect to mixing time. The solid green line is a fit of biexponential growth.

\section{$900 \mathrm{MHz}$}

The same 12 mixing times were used for the 10 micron sample at $900 \mathrm{MHz}$ at a bandwidth of $200 \mathrm{kHz}$ and an $n$ of 512 . The maximum $t_{1}$ for the $900 \mathrm{MHz}$ experiments is less than $3 \mathrm{~ms}$, comparable to a 3 to 4 micron diffusion distance. The 1D spectrum has a FWHM of roughly $6.2 \mathrm{kHz}$. The 2D spectra are shown in figure 6.11, and again have a vertical skew along the $f_{1}$ direction likely due to phase cycling errors. All spectra have have been plotted with a display bandwidth of $40 \mathrm{kHz}$ rather than their full $200 \mathrm{kHz}$ to better observe the broadening 

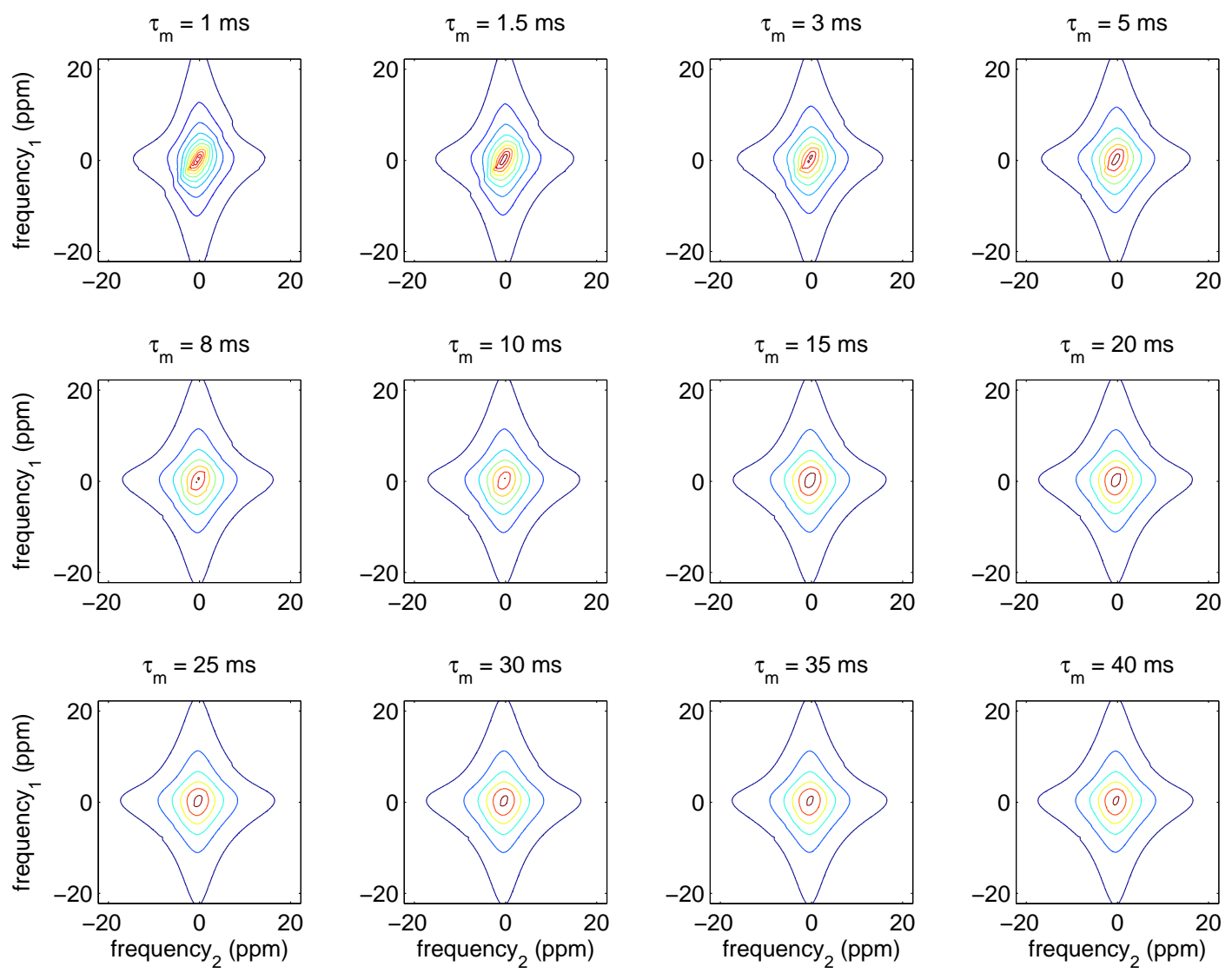

Figure 6.11: $2 \mathrm{D}{ }^{1} \mathrm{H}$ NMR exchange spectra obtained from water in 10 micron bead pack at $900 \mathrm{MHz}$ with a bandwidth of $200 \mathrm{kHz}$. Bandwidth shown in the figure is $40 \mathrm{kHz}$. The 12 examples show mixing times ranging from 1 to $40 \mathrm{~ms}$. Intensities range from $1 \times 10^{-4}$ to $1.1 \times 10^{-3}$ with intervals of $9 \times 10^{-5}$. Blue contour lines indicate lower intensities, with red contour lines indicating higher intensities. 
with respect to increasing mixing time. All 2D spectra have been normalized with respect to integrated intensity to compensate for $T_{1}$ relaxation over the mixing time. Most noticeable in comparing the $400 \mathrm{MHz}$ and $900 \mathrm{MHz}$ spectra is the decrease in noise due to the higher magnetic field. Off-diagonal broadening indicates an increase in off-diagonal intensity. Quantification of this intensity with respect to mixing time is shown in figure 6.12.

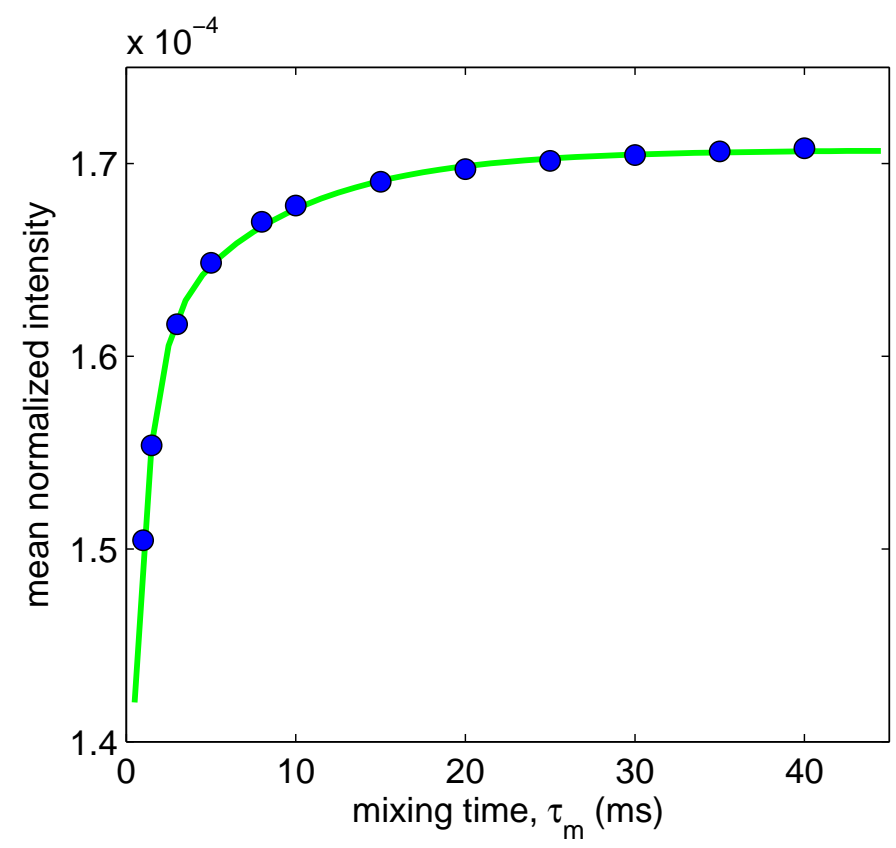

Figure 6.12: Mean normalized intensities located at equidistant points of the center peak along the off-diagonal of $900 \mathrm{MHz}$ spectra shown in figure 6.11 with respect to mixing time. The solid green line is a fit of biexponential growth.

The growth of off-diagonal for the 10 micron beads at $900 \mathrm{MHz}$ shows a trend similar to that found for 10 microns at $400 \mathrm{MHz}$. In both cases characteristic times of $1-2 \mathrm{~ms}$ and $8-18 \mathrm{~ms}$, corresponding to 0.02 and $.05-.08$ bead diameters. However given the large amount of exchange which occurs during the encoding periods, we are cautious in attributing significance to these parameters. 


\subsection{Conclusion}

In this chapter we introduced the first of our experiments, a simple 2D exchange experiment which allowed us to separate the line broadening due to the susceptibility differences between that of the line broadening due to diffusion through an inhomogeneous field. We found that as mixing time is increased, the offdiagonal broadening due to diffusion through the inhomogeneous field is increased. To quantify this off-diagonal broadening we take the mean intensity of two points on either side of the off-diagonal maximum. The two points are chosen as a fraction of the FWHM of the 1D spectrum which are the best compromise between noise and sensitivity to exchange and are plotted with respect to mixing time. We then fit a biexponential to these intensities to obtain characteristic times from which we can deduce a mean displacement to give some insight into the pore size.

In the next chapter, to better obtain information about the pore size, we add a spatial dimension in the form of a propagator which will give $Z$-displacement information about the spins in the pore space directly from experiment rather than from a calculation of characteristic time as we have done in this chapter. 


\section{Chapter 7}

\section{Propagator Resolved Frequency Exchange}

\subsection{Introduction}

In this chapter we move on to an extension of the inhomogeneous local field exchange experiment from the previous chapter and add a third displacement dimension. In the earlier 2D version of the experiment, the off-diagonal intensity arises from all molecules which have changed their local field value, irrespective of the distance travelled. By including the propagator dimension we are now able to distinguish molecules which have diffused different distances along the field gradient axis. Hence, rather than measuring an off-diagonal intensity which is a function of mixing time alone, we add a displacement dimension, allowing a spatio-temporal analysis of the diffusion between sites of differing field. Of course the connection between space and time, in this context, is provided by the diffusion rate, and the characteristic length scale over which local fields change significantly, as measured by the spread of Larmor frequencies in the NMR spectrum. The ability to access the spatial information therefore provides a more robust check on any model used to interpret our experimental results. It is this use of a well-defined spatial dimension which differentiates our method from earlier local field exchange methods such as DDIF or the 2D method described in chapter 6 . 
The new data we acquire consist of two-dimensional spectra where axes are labelled by the frequency spread corresponding to the inhomogeneous field. We then focus our attention to the off-diagonal intensity of these spectra at some particular frequency offset. This is done to establish the degree of exchange that is occurring during the mixing period. We then measure the intensity as a function of molecular displacement, $Z$, and mixing time, $\tau_{m}$, using the label $I\left(Z, \tau_{m}\right)$. We adopt two approaches in the interpretation of our data. First, we model the internal fields in a random bead pack and simulate the restricted diffusive motion of molecules within that pack, thus gaining estimates of $I\left(Z, \tau_{m}\right)$. Second, we adopt a simple analytic model for the migration of NMR signal away from the spectral diagonal by assuming an isotropic Gaussian propagator with effective diffusion coefficient $D_{\text {eff }}$, and a measure of the degree of randomizing of the NMR frequency within the inhomogeneous field spectrum which depends on total displacement $R$ as $1-\exp \left(-R^{2} / 2 l_{c}^{2}\right)$ where $l_{c}$ is some characteristic length scale.

While our new method has strong links with prior work, such as DDIF and $\mathrm{T}_{2}$-exchange methods $[78,39,104,105]$, it is distinct in a number of regards. First it is "many-dimensional", having both evolution and acquisition frequency domains, $\mathrm{f}_{1}$ and $\mathrm{f}_{2}$, a mixing time dimension, and a displacement dimension. Second, the use of pulsed field gradient encoding allows direct determination of molecular displacements, rather than via an indirect deduction based on a known diffusion time and an assumed effective diffusion coefficient, as implied in DDIF. Finally, the multitude of dimensions allows for different modes of analysis, by selecting different "planes" of parameters, or, as we shall show, by comparing with model predictions, we produce a two-dimensional surface, rather than a one-dimensional graph.

\subsection{Method}

\subsubsection{Experimental}

As in the previous chapter, all experiments were performed at $22^{\circ} \mathrm{C}$ on a 400 $\mathrm{MHz}$ Bruker spectrometer using the proton signal from distilled water in a ran- 
dom dense pack of monodisperse glass spheres. The glass beads are the same $100 \mu m$ beads as described in the previous chapter.

\subsubsection{Pulse Sequence}

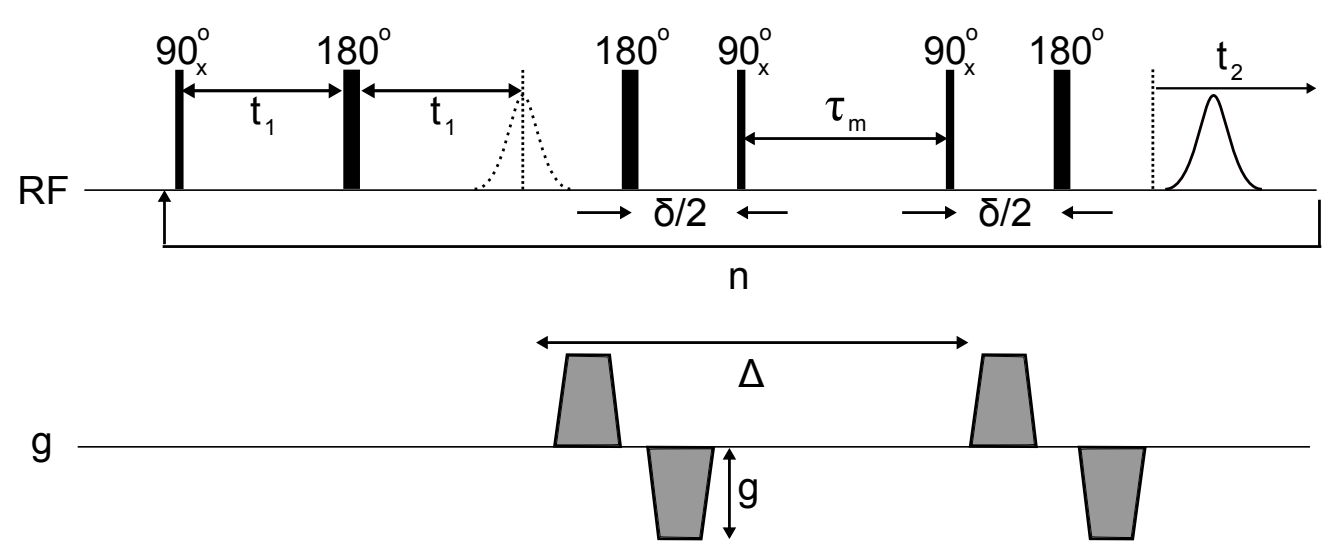

Figure 7.1: Pulsed sequence used for the propagator-resolved field exchange experiment.

The pulse sequence used is shown in figure 7.1. To prevent interaction between the applied gradients and the internal magnetic field gradients of the sample [30], we use bipolar pulsed field gradients of strength $g$ with a storage time $\Delta$ measured between the beginnings of each gradient pair. For each $n$, we step the applied gradients $p$ times to a maximum strength of $13.5 \mathrm{G} \mathrm{cm}^{-1}$ and incrementally increase the frequency encoding time, $t_{1}$ by a increment equal to $1 /$ bandwidth, in our case $(20 \mathrm{kHz})^{-1}$ or $50 \mu$ s. Since we want to keep the storage time $\Delta$ constant for all $n$, we include a spin echo sequence before the diffusion measurement to allow us to increment $t_{1}$. Note that the initial spin echo produced from this spin echo sequence will appear at a time $t_{1}$ after the first $180^{\circ}$. The time between the first and second $180^{\circ}$ is $1.01 \mathrm{~ms}$. Due to the inhomogeneously broadened 1D spectrum, the signal from this echo will have decayed $e^{-1}$ at a $t_{1}$ of $61.2 \mu \mathrm{s}$. Thus we will only forfeit a small amount of resolution in our final 2D spectrum. After the first applied gradient pair, a $90^{\circ}$ pulse stores the magnetization along the $z$ axis to eliminate any effect from $T_{2}$ relaxation, however, the protons still undergo $T_{1}$ relaxation. After a mixing time, $\tau_{m}$, which is 
approximately $\Delta$, the magnetization is brought back to the transverse plane and the second pair of gradients are pulsed to complete the diffusion measurement. We acquire for a time $t_{2}$ following a time $\delta$ after the final $90^{\circ}$ pulse. To ensure the resultant signal is only that derived from the initial $90^{\circ}$ pulse, we utilize a 16 step phase cycling.

This pulse sequence produces an $m \times n \times p$ data matrix where $m$ is the number of acquisition points, $n$ the number of time evolution steps and $p$ the number of q-gradient steps. For all experiments we acquire for 128 points (acquisition time $6.4 \mathrm{~ms}$ ), increment $t_{1} 64$ times (evolution time $3.3 \mathrm{~ms}$ ) and step our applied gradients 33 times for a data matrix of size $128 \times 64 \times 33$ which we zero fill to $256 \times 256 \times 64$ and apply a 3D fast Fourier transform. The total experiment time is about 15 hours.

The one-dimensional NMR spectrum for the water/beadpack sample is shown in figure 7.2a, and has a full width half maximum (FWHM) of $5.2 \mathrm{kHz}$ or 12.9 ppm.
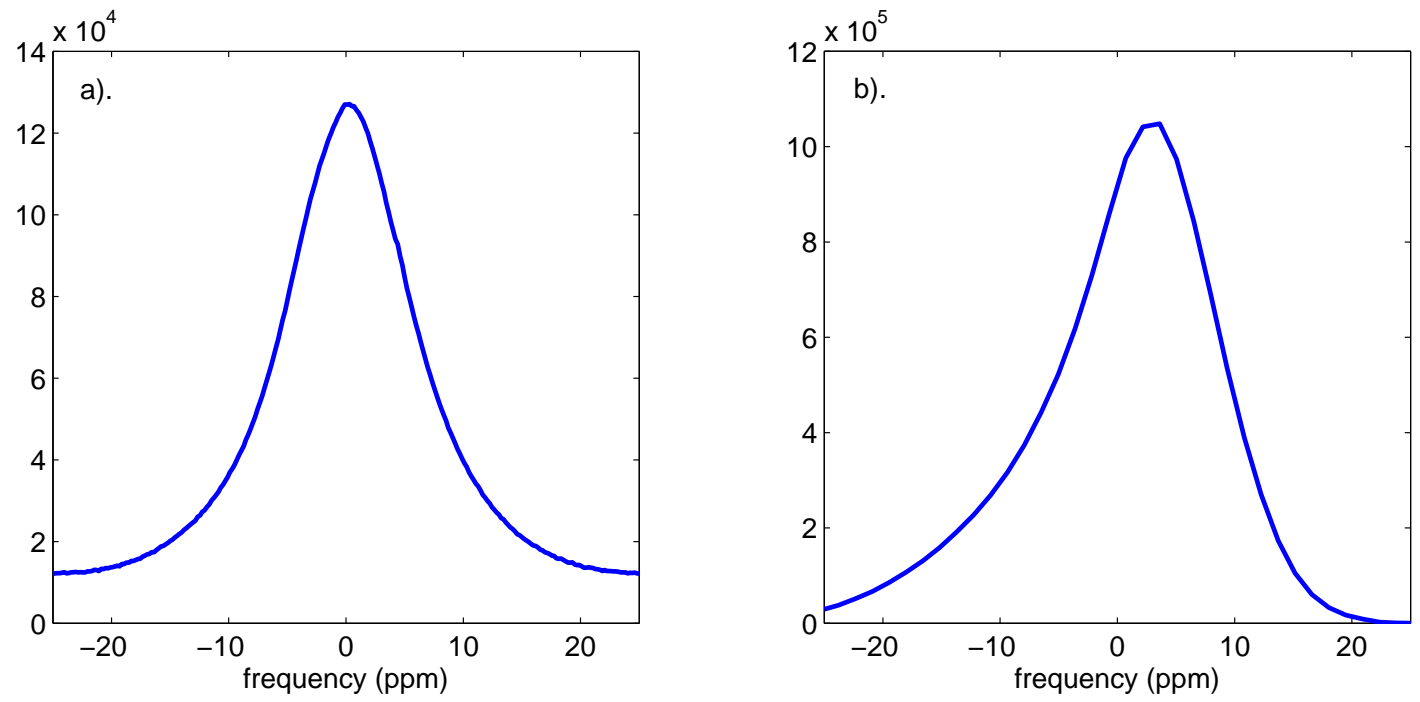

Figure 7.2: a). The measured $1 \mathrm{D}^{1} \mathrm{H}$ NMR spectrum for the water in glass bead pack at $400 \mathrm{MHz}$. The spectrum has an FWHM of $5.2 \mathrm{kHz}(12.9 \mathrm{ppm})$. b). The simulated NMR spectrum for a random bead pack. Frequency units are dimensionless and the spectrum has an FWHM of 0.91 (13 ppm). Both 1D spectra are plotted in units of ppm for easy comparison. 


\subsection{Experimental and Simulation Results}

The simulation used in this chapter is previously described in chapter 5. In it, we generate an internal magnetic field by placing a $z$-aligned magnetic dipole at the center of each sphere in a matrix of randomly packed spheres. We then allow approximately 12.4 million tracers to diffuse through the pore space of the system, for a tracer density of approximately 80 tracers per $10 \mu \mathrm{m}^{3}$. The reason for using such a high density of tracers is due to the fact that we will be separating tracers in to bins with respect to their $Z$-displacement distances, therefore, we need to have a large amount of tracers to ensure an adequate amount of tracers are represented in each $Z$-displacement. After diffusion, the magnetic field at the final position for each tracer is recorded. From the initial and final positions of the tracers, we are able to simulate a propagator, which is essentially a histogram of tracer $Z$-displacements in the program. Using knowledge of these $Z$-displacements, we can construct simulated 2D exchange spectra which are comprised only of tracers which have diffused a certain $Z$-displacement.

Figure 7.3 shows the $Z$-displacement propagator for a mid-range mixing time of $160 \mathrm{~ms}$. This corresponds to a $\Delta$ of $162 \mathrm{~ms}$. The experimentally measured propagator is shown by the solid line and has a resolution of $3.5 \mu \mathrm{m}$. For molecules diffusing in a random pore glass with pore separation $b$, the expected echo attenuation is the diffusive-diffraction behavior, as given in the pore hopping model [106] by

$$
E(q, \Delta)=\left|S_{0}(q)\right|^{2} \exp \left(-\frac{6 D_{e f f} \Delta}{b^{2}}\left[1-\frac{\sin (q b)}{q b}\right]\right)
$$

where $\left|S_{0}(q)\right|^{2}$ is the pore structure factor and the $q$ vector magnitude is $\gamma \delta g$. For asymptotic conditions, $D_{\text {eff }} \Delta \gg b^{2}$, this expression reduces to $\exp \left(-q^{2} D_{\text {eff }} \Delta\right)$ for which the asymptotic propagator is given by the Gaussian

$$
P(Z, \Delta)=\left(4 \pi D_{e f f} \Delta\right)^{-1 / 2} \exp \left(-\frac{Z^{2}}{4 D_{e f f} \Delta}\right)
$$

where $Z$ represents displacements along the gradient axis.

Given $\Delta=162 \mathrm{~ms}$, and fitting to the low $q^{2}$ limit of the echo attenuation $E(q)$ (i.e. from the mean-squared displacement $D_{\text {eff }}=\left\langle Z^{2}\right\rangle / 2 \Delta$ ), we find an 


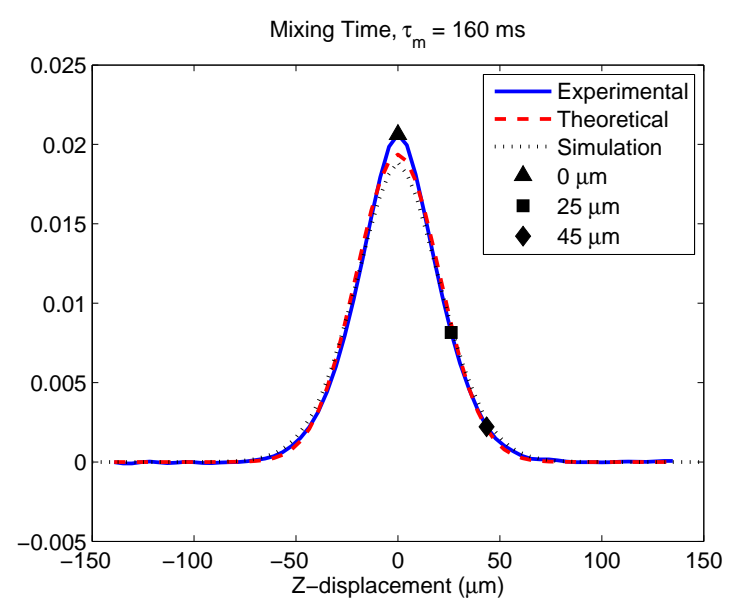

Figure 7.3: Propagator for a mixing time of $160 \mathrm{~ms}$. The experimental data is shown by the solid line. The calculated theoretical propagator as given by equation 7.2 using an effective diffusion of $0.65 D_{0}$ is shown by the dashed line. The simulated propagator is shown by the dotted line. The markers and indicate $Z$-displacements of $0 \mu \mathrm{m}, 25 \mu \mathrm{m}$, and $45 \mu \mathrm{m}$ respectively. The experimental and theoretical propagators have a resolution of about $8.7 \mu \mathrm{m}$, but the echo attenuations are zero filled to 64 points before Fourier transformation. 
effective diffusion of $D_{\text {eff }}=0.65 D_{0}$ where $D_{0}$ is the diffusion coefficient of bulk water. The theoretical propagator is shown by the dashed line and agrees moderately well with experiment. The slightly different experimental propagator shape probably arises because the asymptotic conditions for diffusion have not quite been reached at $160 \mathrm{~ms}$, and therefore the actual propagator is not yet gaussian. The dotted line is the propagator found by simulation at a mixing time of $160 \mathrm{~ms}$. Again, to determine an effective diffusion coefficient, we derive $E(q)$ from the simulated propagator and fit for $q^{2}$ in the low $q$ limit. For the simulations, the effective diffusion coefficient at $162 \mathrm{~ms}$ is found to be $0.82 D_{0}$ where $D_{0}$ is the bulk diffusion constant.

Figure 7.4 shows the approach to asymptotic conditions as the mixing time is increased of $D_{\text {eff }}$ for both experiment and simulations. For both the experiments and simulation, asymptotic conditions have not been fully reached at 640 $\mathrm{ms}$, however the limit is sufficiently close that a reasonable estimate can be made for the purpose of calculating porosity. In a bead pack the expected asymptotic diffusion coefficient is $\sqrt{\phi} D_{0}$ where $\phi$ is the porosity [58]. For the experiments, where we find $\phi=0.436$, this agrees well with this expected limit. The simulated bead pack has a porosity of 0.45 , suggesting an asymptotic $D_{\text {eff }}$ of $0.67 D_{0}$. This estimate is slightly lower than what is shown in figure 7.4, and suggests that asymptotic conditions have not yet been reached at $162 \mathrm{~ms}$ or $642 \mathrm{~ms}$ in the case of the simulations. It is possible that the size of the simulated cylindrical bead pack may have been insufficient to obtain a representative elementary volume. In fact, we find the average of the $B_{z}$ field in figure $7.2 \mathrm{~b}$ to be 0.0146 suggesting we do not have full anisotropy in the sample, as full anisotropy would give us an average $B_{z}$ of 0 . We are constrained by computer memory with regards to the size of this sample, however the simulations presented do provide a useful comparison.

We can select 2D spectra along the propagator dimension which correspond to spectra containing the NMR signals from molecules which have diffused a specific range of $Z$-displacements. The top row of figure 7.5 shows the experimental spectra obtained at $\tau_{m}=160 \mathrm{~ms}$ for $Z$-displacements of $0 \mu \mathrm{m}, 25 \mu \mathrm{m}$, and $45 \mu m$ which correspond to the markers $\Lambda, \mathbf{\square}$, and $\downarrow$ on the experimental propagator in figure 7.3. 

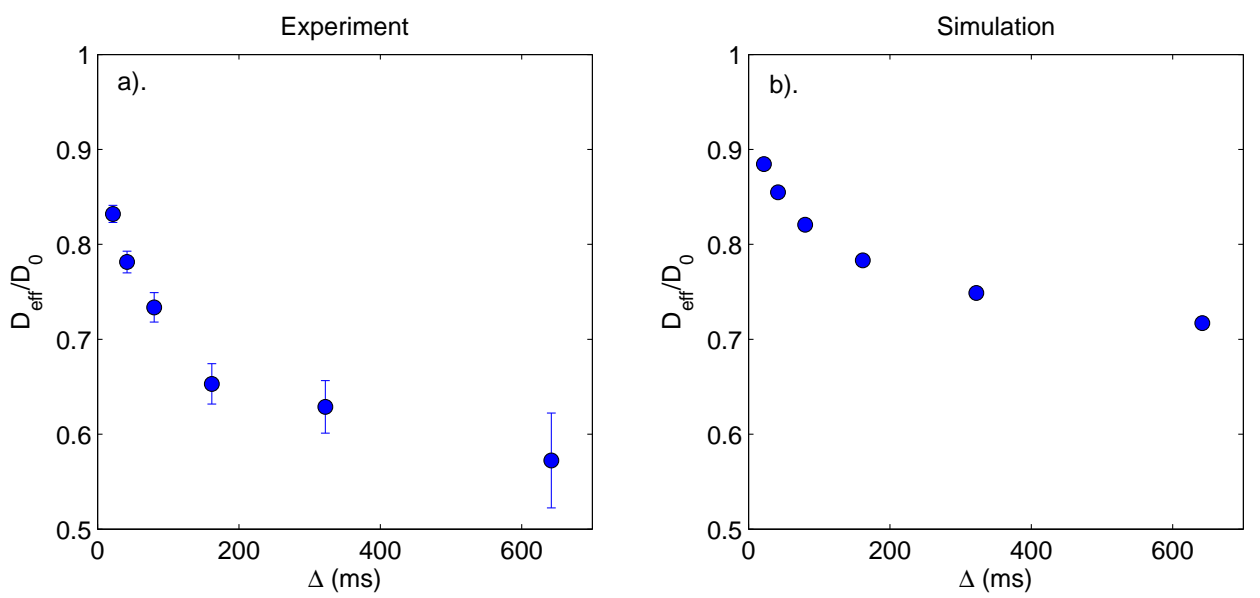

Figure 7.4: a). The ratio of effective diffusion to bulk diffusion for a $100 \mu \mathrm{m}$ beadpack from experiment using the pulse sequence shown in figure 7.1 using a maximum gradient of $5 \mathrm{G} / \mathrm{mm}$ and without $t_{1}$ stepping. $\mathrm{b}$ ). The ratio of effective diffusion to bulk diffusion for the simulation. Note error bars are inside the markers.

Since a molecule diffusing for a greater distance has a greater chance of changing its local magnetic field value, we expect the off-diagonal of the spectrum to grow in intensity with increasing displacement. In the top row of figure 7.5, we see that for a $Z$-displacement of $0 \mu \mathrm{m}$, there is a narrow off-diagonal. Of course, zero $Z$-displacement does not mean that a spin-bearing molecule has not changed its local field during $\tau_{m}$. The possibility remains that that some molecules will have diffused to a different field in the transverse $(X, Y)$ displacement plane during $\Delta$, but remained at or returned to their initial $z$-position at the end of the diffusion measurement. This will result in some broadening along the off-diagonal even at $0 \mu \mathrm{m} Z$-displacement. Increasing $\tau_{m}$ (and hence $\Delta)$ makes such broadening even more pronounced as $X$ and $Y$ displacements grow.

To quantify the off-diagonal intensity for each $\tau_{m}$ and $Z$, we average together two points on either side of the maximum of the off-diagonal slice as we did in the previous chapter. These two points are chosen to be the equivalent of a fraction of the FWHM of the 1D inhomogeneous spectrum. In this chapter, we will look at two different widths, 0.8 FWHM and 1.0 FWHM, the equivalent of 

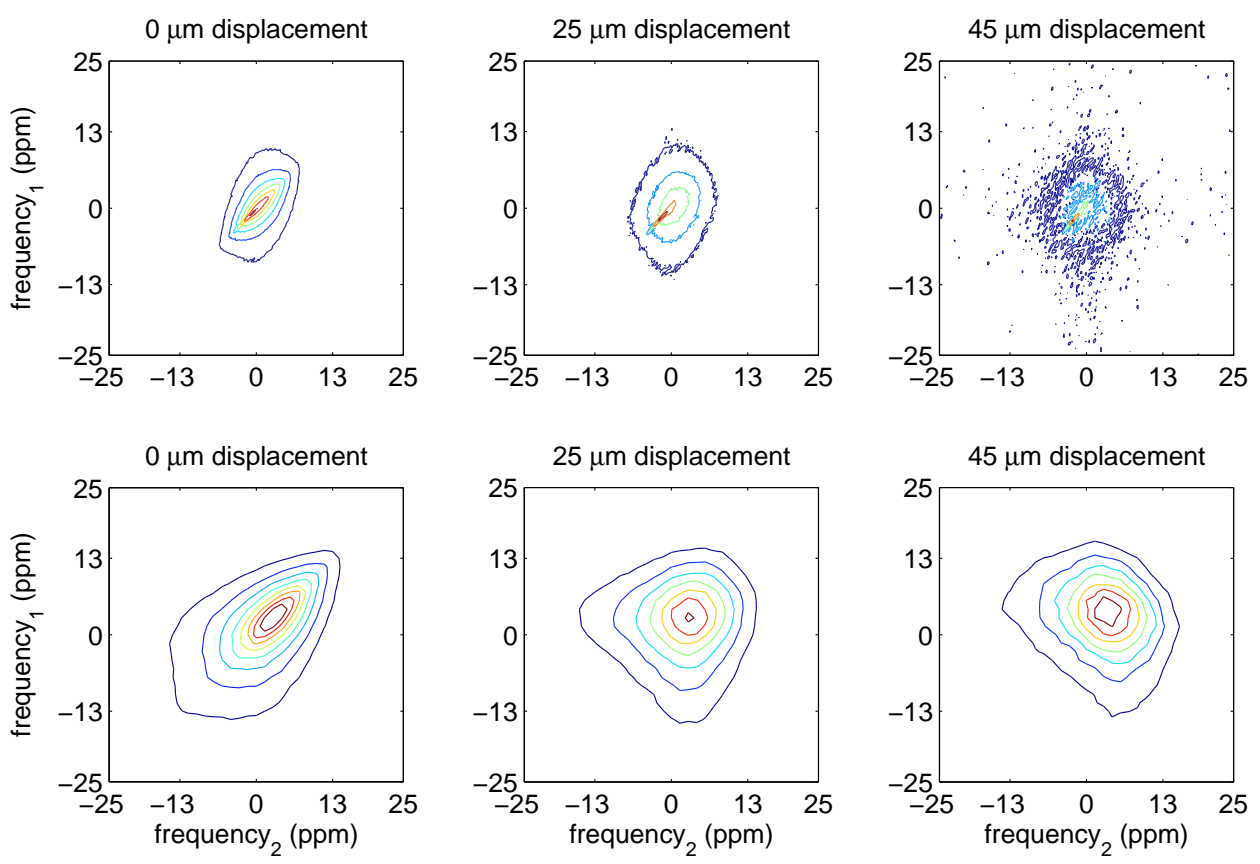

Figure 7.5: 2D spectra for a $\tau_{m}$ of $160 \mathrm{~ms}$. Top row: obtained using experimental data. Intensities range from $5 \times 10^{-5}$ to $1 \times 10^{-4}$ in increments of $4.5 \times 10^{-5}$. Bottom row: obtained using simulated data. Intensities range from $5 \times 10^{-4}$ to $4 \times 10^{-3}$ in intervals of $4.4 \times 10^{-4}$. Red contour lines indicate higher intensities. 
$4.3 \mathrm{kHz}(10.75 \mathrm{ppm})$ and $5.2 \mathrm{kHz}(12.9 \mathrm{ppm})$. This choice is consistent with the compromise between the signal to noise ratio and the sensitivity to exchange as found previously. The average intensities at these offsets are plotted in figure 7.6a-b as a function of both $\tau_{m}$ and $Z$, referred to as $I\left(Z, \tau_{m}\right)$.

a). 0.8 FWHM, Experiment

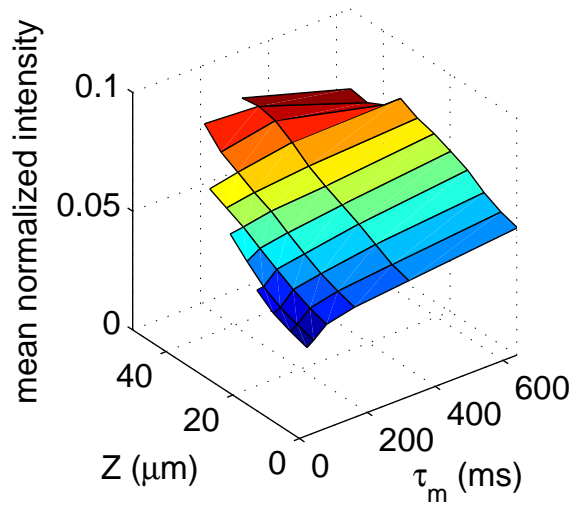

c). 0.8 FWHM, Simulation

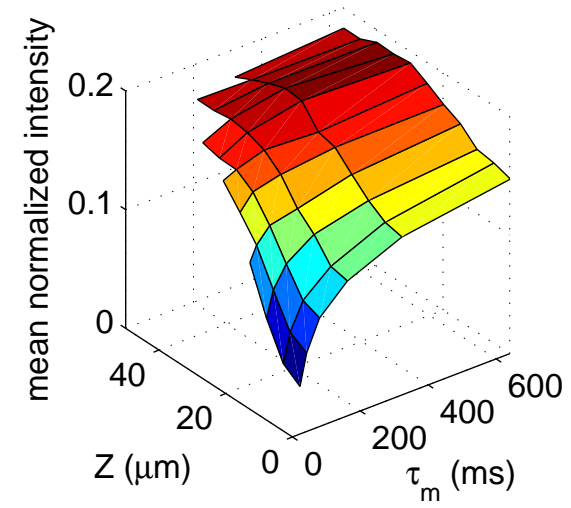

b). 1.0 FWHM, Experiment

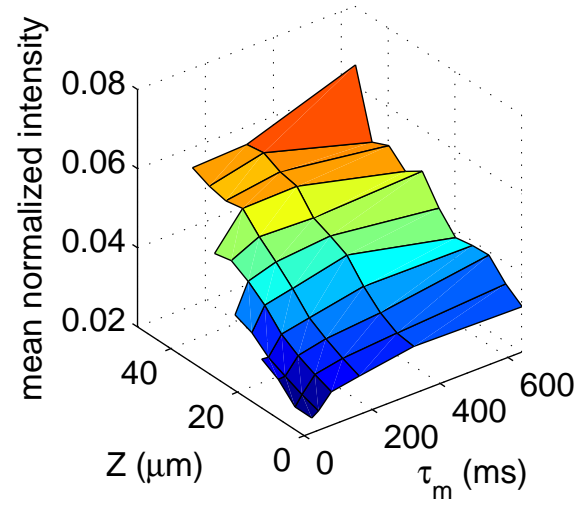

d). 1.0 FWHM, Simulation

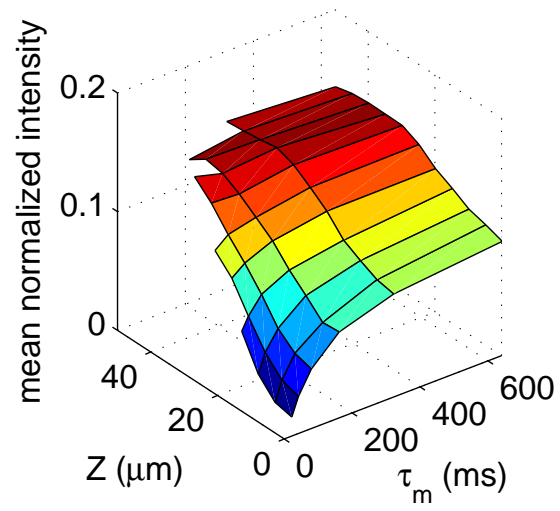

Figure 7.6: $I\left(Z, \tau_{m}\right)$ for a $\tau_{m}$ of $160 \mathrm{~ms}$ and at different displacements $Z$. The spectra are normalized to a common total intensity in order to make a visual comparison. a). For experimental data at 0.8 FWHM. b). For experimental data at 1.0 FWHM. c). For simulated data at 0.8 FWHM. d). For simulated data at $0.8 \mathrm{FHWM}$. The blue colors indicate lower intensities while the red colors indicate higher intensities.

Mean normalized intensities increase as both $\tau_{m}$ and $Z$ increase. Off-diagonal 
line broadening occurs because water molecules, whose spectra were acquired during $t_{1}$ evolution encoding (a total period of up to $3.3 \mathrm{~ms}$ ) are diffusing through inhomogeneous fields to a new local field value at the time of acquisition (which occurs over $6.4 \mathrm{~ms}$ ). For the purpose of the present analysis we ignore any motional averaging spectral effects which may result from the finite evolution and acquisition times, since these are generally lower than the $\tau_{m}$ values used in this work. Of course, we expect an increase of off-diagonal intensity with increasing $Z$ since the increase in positional displacement increases the possibility of a water molecule having moved to a different local field. For increasing $\tau_{m}$, both $Z$ and $X, Y$ displacements contribute, further increasing the likelihood of an altered local field, and thus increasing off-diagonal intensity, $I\left(Z, \tau_{m}\right)$.

The simulated propagator-resolved 2D spectra are calculated by including only those particles which have diffused a designated $Z$-displacement within the bead pack. The bottom row of figure 7.5 shows the calculated simulated spectra for a $\tau_{m}$ of $160 \mathrm{~ms}$, and $Z$ of $0 \mu \mathrm{m}, 25 \mu \mathrm{m}$, and $45 \mu \mathrm{m}$. Also shown, in figure 7.6c-d, are the off-diagonal $I\left(Z, \tau_{m}\right)$ data obtained from the simulation at 0.8 FWHM offset and 1.0 FWHM offset. The addition of displacement information allows us to develop a theory incorporating both mixing time and displacement, rather than extracting exchange times by simple single or double exponential fitting, as in the previous method.

In the previous chapter, where no spatial resolution was incorporated, all spins were included no matter what their displacements. By integrating the current data over all $Z$ we should be able to approximate the off-diagonal intensity growth expected for the non-spatially resolved method. Figure 7.7a shows the result for both experimental FWHMs from figure 7.6a-b, and figure 7.7b shows the result for both simulated FWHMs from figure 7.6c-d. In chapter 6, we were able to achieve shorter mixing times, down to $1 \mathrm{~ms}$, because of the absence of displacement-encoding gradient pulses. In the present work, these timing constraints enforced a lower mixing time limit of $20 \mathrm{~ms}$. For times above this, both sets of data are comparable. In both cases we have attempted fits to an exponential rise. Here, for the more limited range of $\tau_{m}$ values available, we fit a single exponential of the form $\int I\left(Z, \tau_{m}\right) d Z=A \exp \left(-\tau_{m} \tau^{-1}\right)+C$ and obtain characteristic times of $83 \mathrm{~ms}$ for both the 0.8 FWHM and 1.0 FWHM widths for the 

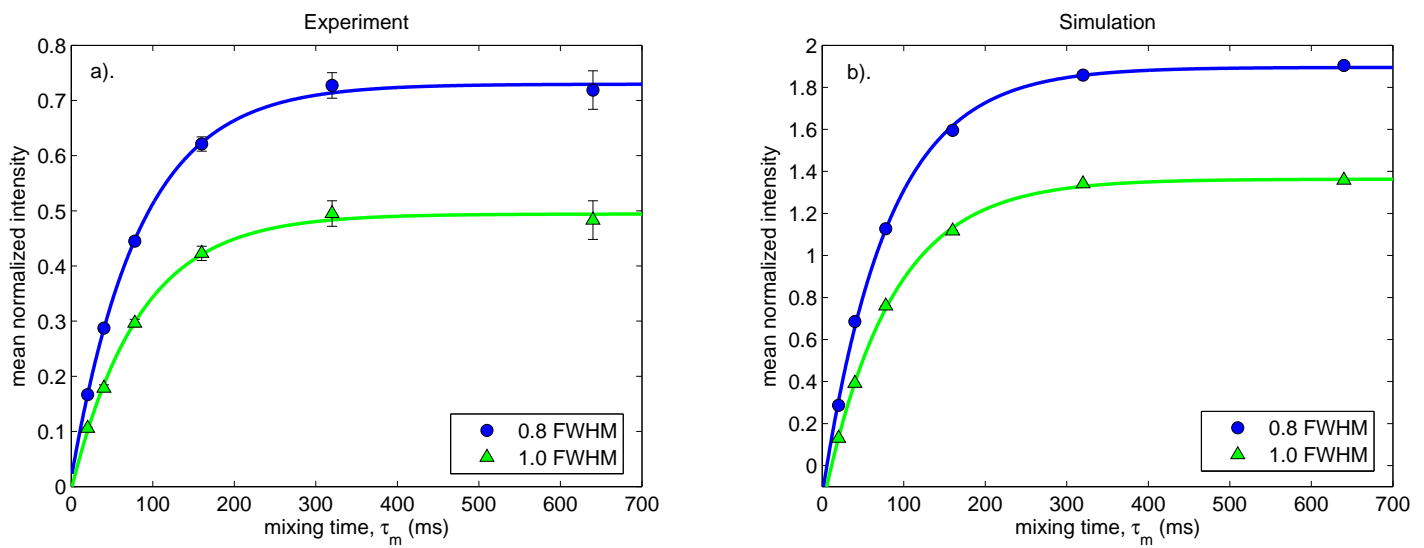

Figure 7.7: a). Mean normalized off-diagonal intensities summed for all $\Delta Z$. The solid lines represent a single exponential curve fit to $\int I\left(Z, \tau_{m}\right) d Z=$ $A \exp \left(-\tau_{m} / \tau\right)+C$. b). Simulated mean normalized off-diagonal intensities integrated over all $\Delta Z$. The solid lines represent a fit to the same function as in a). Note in b). error bars are smaller than the data markers.

spatially resolved method, which corresponds to a mean squared displacement of 0.18 bead diameters. For the non-spatially resolved method, we obtain the characteristic times of 402 and 870 ms respectively. These characteristic times correspond to a range of 0.40 and 0.59 bead diameters. Indeed these exponential fits are not particularly good, and may not be justified by theory, as we shall later demonstrate.

In the case of the simulations, off-diagonal intensities are obtained from 2D spectra in a similar fashion to the experiments. Note in figure $7.6 \mathrm{c}-\mathrm{d}$, that the minimum $\tau_{m}$ simulated is $20 \mathrm{~ms}$. At shorter mixing times, off-diagonal intensities were too weak and dominated by noise. As in the case of the experiments (figure 7.6a-b), the simulated intensities shown in figure 7.6c-d exhibit a growth of intensity with increasing $\tau_{m}$ and $Z$.

Again, integrating the simulated data over all $Z$ for each $\tau_{m}$ also results in a growth similar to that calculated experimentally as in figure 7.7a. Figure 7.7b shows the simulated curves fit to the same expression from which we obtain characteristic times of $80 \mathrm{~ms}$ and $84 \mathrm{~ms}$ for 0.8 FWHM and 1.0 FWHM respectively. These characteristic times correspond to mean squared displacements of around 0.18 bead diameters. 


\subsection{Theory}

We assume that the mean intensity, $I\left(Z, \tau_{m}\right)$, of the off-diagonal grows as molecules diffuse to the new local fields. We define, for convenience, a soft well given by $\left(1-\exp \left(-R^{2} / 2 l_{c}^{2}\right)\right)$, where $l_{c}$ is a characteristic length (see figure 7.8). This function represents the conditional probability of a molecule changing its local field so as to contribute to the off-diagonal intensity. It is zero for $R=0$ and 1 as $R \rightarrow \infty$ as required. In the $Z$-resolved experiment, $R^{2}$ is the total distance a molecule has diffused such that it ends on a plane of fixed displacement, $Z$, along the gradient direction, $Z$. In other words, $R^{2}=Z^{2}+r^{2}$ where $r$ is the distance traveled transverse to the magnetic field gradient. Given an a priori knowledge of $Z$ at any time $\tau_{m}, I\left(Z, \tau_{m}\right)$ is given by

$$
\begin{aligned}
I\left(Z, \tau_{m}\right) & \propto \int_{0}^{\infty} 2 \pi r d r\left(4 \pi D_{e f f} \tau_{m}\right)^{-1} \exp \left(-\frac{r^{2}}{4 D_{e f f} \tau_{m}}\right)\left(1-\exp \left(-\frac{Z^{2}+r^{2}}{2 l_{c}^{2}}\right)\right) \\
& =\text { const } *\left(1-\frac{2 l_{c}^{2}}{2 l_{c}^{2}+4 D_{\text {eff }} \tau_{m}} \exp \left(-\frac{Z^{2}}{2 l_{c}^{2}}\right)\right) .
\end{aligned}
$$

Weighting $I\left(Z, \tau_{m}\right)$ by the propagator $P\left(Z, \tau_{m}\right)$ and integrating over $Z$, we obtain the expected result for the non-spatially resolved experiment,

$$
I\left(\tau_{m}\right)=\text { const } *\left(1-\left[\frac{2 l_{c}^{2}}{2 l_{c}^{2}+4 D_{e f f} \tau_{m}}\right]^{3 / 2}\right) .
$$

Interestingly, this function differs considerably from the naïve exponential rise assumed earlier and represents the experimental data with fewer fit parameters, as shown in figure 7.9a, where a fit to $l_{c}$ yields $21 \mu \mathrm{m}$ and $22 \mu \mathrm{m}$ for both 0.8 FWHM and 1.0 FWHM, respectively. We repeat this fit for the simulated data as shown in figure $7.9 \mathrm{~b}$ and obtain an $l_{c}$ of 23 to $25 \mu \mathrm{m}$ for 0.8 FWHM and 1.0 FWHM respectively. This characteristic length will give us an idea of distance a molecule needs to travel in order to sample a significant range of local fields.

We might expect the "propagator-summed" data presented in figures 7.7 and 7.9 to be equivalent to data obtained in an experiment with no propagator dimension, as in chapter 6 where we used a different pulse sequence in which no 


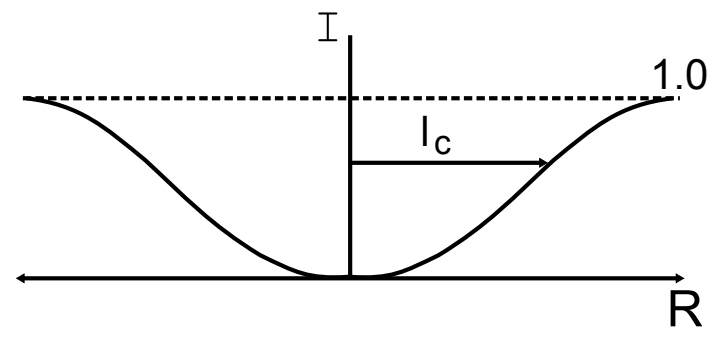

Figure 7.8: A softwell of $\left(1-\exp \left(-R^{2} / 2 l_{c}^{2}\right)\right)$, with a characteristic length $l_{c}$.
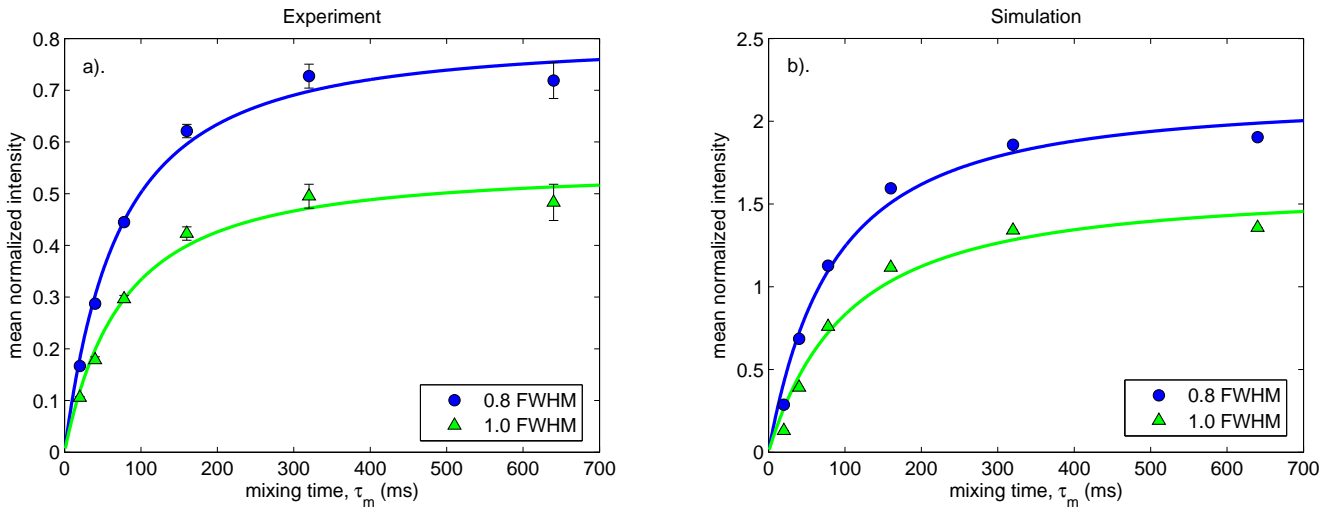

Figure 7.9: a). Calculated off-diagonal intensities from experiment using equation 7.4 with a characteristic length of $21 \mu \mathrm{m}$ and $22 \mu \mathrm{m}$ for 0.8 FWHM and 1.0 FWHM respectively. b). The same as a). but with simulated data. Characteristic lengths are 21 and $32 \mu m$ for 0.8 FWHM and 1.0 FWHM respectively. 
gradient pulses were included. However, the fit to that earlier data using equation 7.4 results in a much shorter length scale, $l_{c} \simeq 6 \mu \mathrm{m}$. This discrepancy may be due to motional averaging effects during frequency encoding. We note that our earlier method used a longer frequency encoding time (and hence greater spectral resolution) than that employed in the present work (12.8 ms compared to $6.4 \mathrm{~ms}$ total $t_{2}$ acquisition time and 12.8 compared to $3.2 \mathrm{~ms}$ total $t_{1}$ evolution time). We should observe that the soft well model which led to equation 7.4 is simplistic and does not accurately represent the complexity of the local field distribution. For example, we can see from figure 7.10 that we have a large change in field in the pore throats, which corresponds to a large local magnetic field gradient. A water molecule does not have to diffuse a large distance to experience a large change in magnetic field and thus a corresponding increase in off-diagonal intensity. This means that the ensemble of molecules will exhibit multiple length scales for field exchange, depending on starting position. Hence it is not surprising that different pulse sequences may return different length scales. However we do find internal consistency within the pulse sequence used here, when we compare fits for propagator-summed and propagator-resolved experiments.

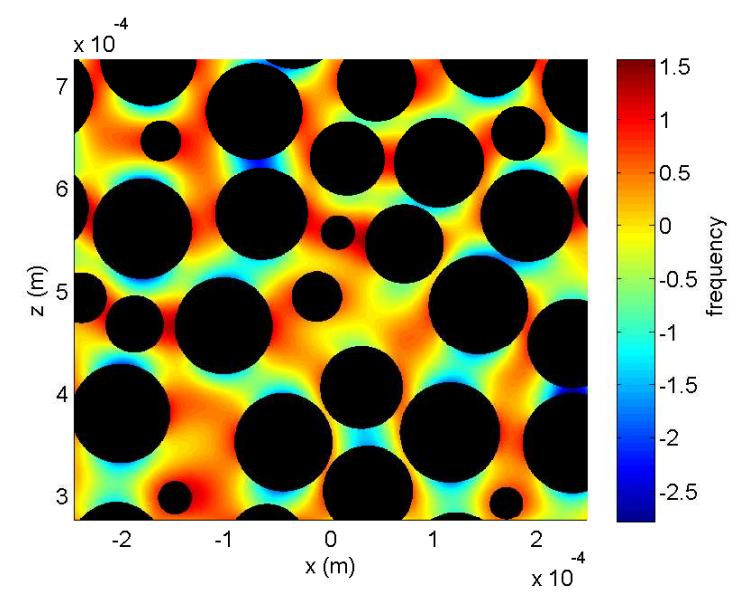

Figure 7.10: The simulated internal magnetic field of an $x z$-plane slice centered at $y=0$.

Using the effective diffusion coefficient measured for for each $\tau_{m}$, and our $Z$-displacements which range from approximately 0 to $50 \mu m$, we plot $I\left(Z, \tau_{m}\right)$ as given by equation 7.3 in figure 7.11. The best representation of our experimental and simulated 2D surfaces shown in figure 7.6 is found by choosing $l_{c}$ in 
the range 20 to $30 \mu \mathrm{m}$. An $l_{c}$ value of around $20 \mu \mathrm{m}$ is comparable with a typical pore dimension in a $100 \mu \mathrm{m}$ diameter bead pack. We note that Stapf [107] found correlation lengths of 0.35 - 0.4 bead diameters for simulated diffusion in a monodisperse bead pack. Audoly et al [74] suggest that it is the pore dimension which provides the relevant length scale for internal field variations. Finally, we note that the correspondence between $l_{c}$ values obtained for both the experiment and the simulation found in our study is encouraging, and provides some support for the simple physical concepts used in the analysis of our results.

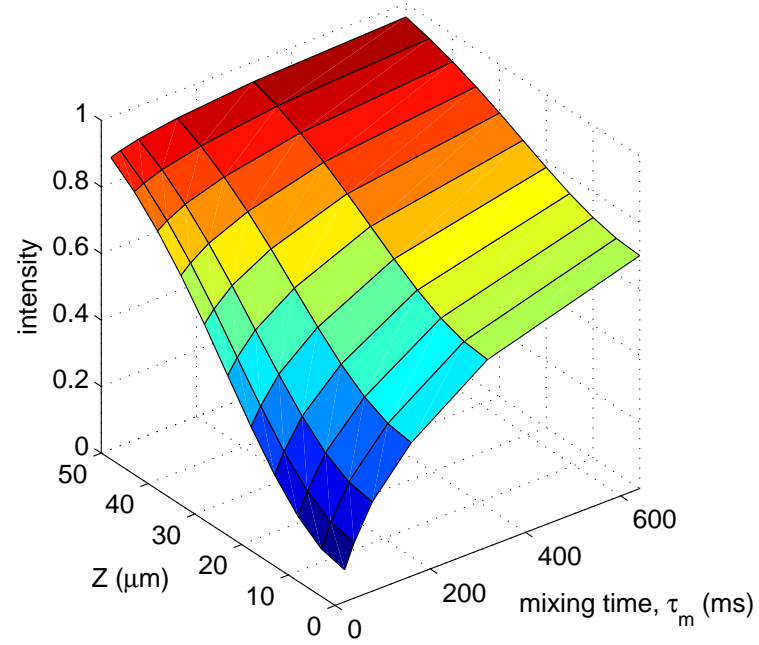

Figure 7.11: Calculated off-diagonal intensities using equation 7.3 with a characteristic length of $25 \mu \mathrm{m}$.

\subsection{Conclusions}

In this chapter, we present results of a propagator-resolved field exchange experiment. By adding a displacement dimension to the previous frequency exchange experiment, we are able to separate 2D spectra by both spatial displacement and mixing time rather than just by mixing time alone. We find offdiagonal intensities increase with increasing $Z$ and $\tau_{m}$ which is to be expected. 
The results of a simulation also produce similar results. A simple theory based on a characteristic length over which significant changes in local field occur, gives good agreement with both experiment and simulations. The new insight here concerns the use of a spatio-temporal approach which has the advantage of yielding a characteristic length from an exchange experiment in a more natural manner. 


\section{Chapter 8}

\section{$T_{2}-B_{z}^{i}$ Correlation}

\subsection{Introduction}

Two-dimensional correlation experiments allow us to correlate two material properties, for example, answering the question as to what diffusion coefficients correspond to what transverse relaxation values in a system. The correlation spectrum can give us information about the structure of the sample.

Diffusion-relaxation correlation spectroscopy (DRCOSY) allows us to correlate $T_{2}$ with corresponding diffusion coefficients. This can enable us to distinguish between two fluids such as oil and water [47] or can allow us to determine where water is located within a porous medium such as hollow capsules [45] or plant structures [46]. For example, the $T_{2}$ distributions of oil and water are often similar, but their corresponding diffusion distributions are different since water typically diffuses faster than oil. By correlating $D$ and $T_{2}$, we are able to separate the water or oil signal contribution in the $T_{2}$ distribution, thus allowing one to distinguish between the two fluids.

Relaxation-relaxation correlation spectroscopy (RRCOSY) [48] correlates $T_{1}$ with $T_{2}$ which provides another way to investigate the pore space in porous media. This method has been used to study enzymes [49], muscle tissue [50], water filled rocks [51], and cements [37, 38]. By correlating $T_{1}$ with $T_{2}$, one can find the $T_{1} / T_{2}$ ratio, which provides information about the rotational mobility of a molecule, and which can also provide information about the exchange of 
protons within a porous system.

In this chapter we present a simple method which correlates the inhomogeneous internal magnetic field with the $T_{2}$ relaxation. By using a CPMG echo train in which the echo decay is also acquired, we can find the $T_{2}$ values that correspond to specific frequency offsets. A related experiment was carried out in 2002 by Sun and Dunn [83] where the $T_{2}$ and internal magnetic field gradient, $g$ were correlated. They found that the largest gradients in a sample correspond to the smallest pores. We know from our simulation and simulations by others [74] that the change in the shape of the internal magnetic field within the pore space of a monodisperse bead pack is independent of pore and grain size. However, by varying the echo spacing in our CPMG echo train, we can obtain information about the internal field gradients, which we know are dependent on pore and grain size. Beginning with a simulated cubic pack of 6 beads, then building to our simulated random bead pack as described in section 5.2, we show how the shape of a correlation between the magnetic field offset and the magnitude of the magnetic field gradient gives an indication of sample pore shape, grain size, pore size heterogeneity and grain heterogeneity. We then move on to running a $T_{2}-B_{z}^{i}$ correlation experiment with varying $\tau$ using a simple sample of a monodisperse bead pack and a more complicated sample of a Bentheimer sandstone, and find confirmation of our simulated results.

\subsection{Simulating the $|g|-B_{z}^{i}$ Correlation}

We first start with a series of simplistic 6 bead structures to create a pore whose size and anisotropy we can control. We use four different bead packs, shown in the top row of figure 8.1. From left to right, we have a small pore using closely packed beads, a larger pore using loosely packed beads, an anisotropic pore with a horizontal long axis created by shifting the four outer beads oriented parallel to the $x y$-plane out and moving the two beads parallel to the $z$-axis closer together, and finally, we have an anisotropic pore with a vertical long axis created by moving the beads parallel to the $y$ - and $z$-axis outwards and moving the beads parallel to the $x$-axis inwards. These beads allow us to see how the 
internal magnetic field and the magnitude of the magnetic field gradient are correlated when we change properties of the pore such as pore size and pore anisotropy.
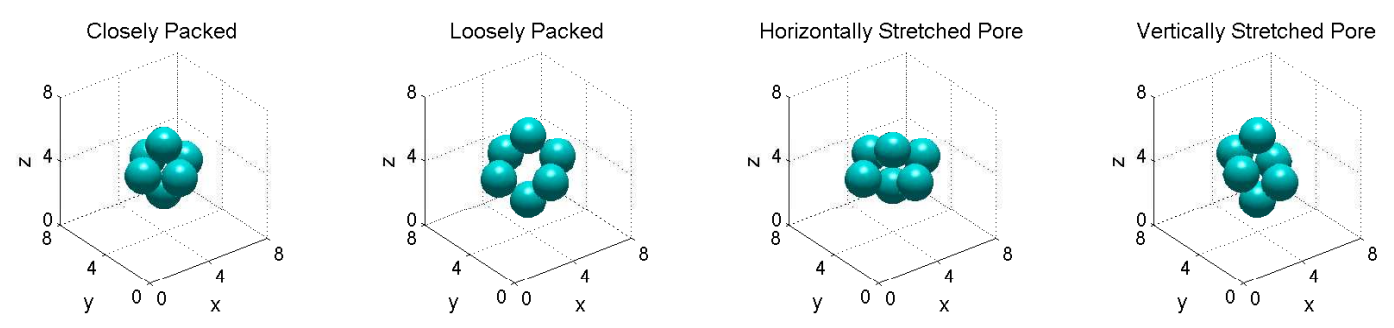

Closely Packed, Rotated

Loosely Packed, Rotated
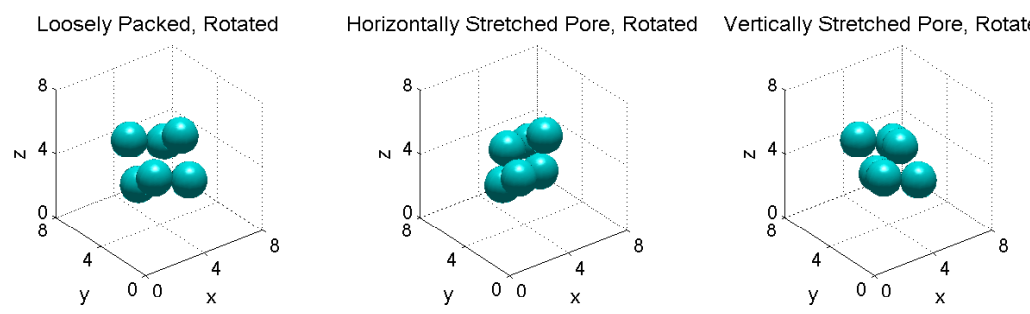

Figure 8.1: Top row: The four simple bead packs used which are comprised of 6 spheres each. From left to right the structures are: closely packed (small pore), loosely packed (large pore), horizontally stretched pore, and vertically stretched pore. Bottom row: As for the top row but the bead packs are rotated so that the beads previously parallel to the $z$-axis now lie along the body diagonal of a cube of side 8 centered about $(4,4,4)$.

The corresponding field and gradient maps for the bead packs in the top row of figure 8.1 are shown in figure 8.2. The field maps are shown in the top row, and the gradient magnitude maps are shown in the bottom row. In the field maps, we see that for the closely packed and loosely packed shape, the magnetic field offset is zero at the center of the pore which is to be expected. However in the horizontally and vertically stretched pores we either have a strongly negatively offset field as in the horizontally stretched pore, or a strongly positive offset field as in the vertically stretched pore. We can correlate the simulated field and gradient, and these correlations are shown in figure 8.3. What these correlations tell us is that the diagonal "wings" which protrude off the edge of the spectrum correspond to regions of high gradient intensity which occur in 

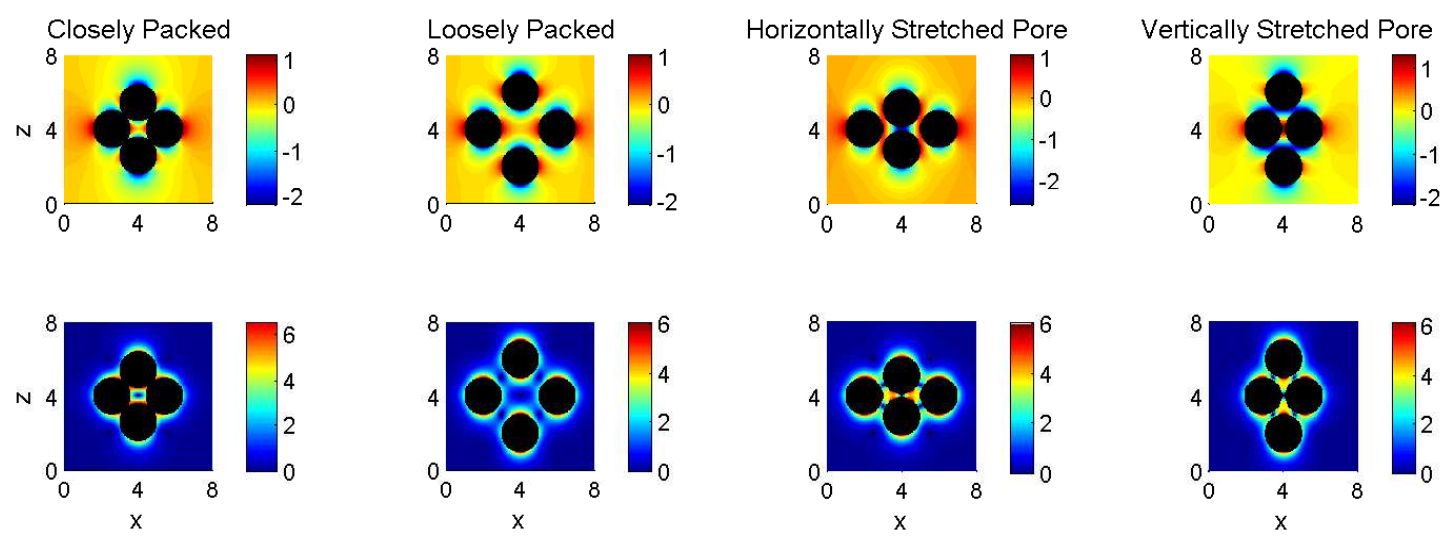

Figure 8.2: Top row: Simulated magnetic field maps for the bead packs shown in the top row of figure 8.1. Bottom row: The simulated magnetic gradient field magnitude for the same bead packs.

regions of large magnetic field offsets. Comparing figures $8.3 a$ and $b$, we see that the $|g|-B_{z}^{i}$ correlations could possibly be indicative of pore size, as the diagonal wings corresponding to the smaller pore are more pronounced than those which correspond to the larger pore. Similarly, comparing figures $8.3 \mathrm{c}$ and $\mathrm{d}$, we see that the diagonal wings on the correlations could be indicative of pore anisotropy, as a horizontally stretched pore results in high gradients correlated with large negative offsets and vice versa for vertically stretched pores.

To make the pores more complex, we rotate the bead packs so that the beads which were originally parallel to the $z$-axis are now aligned along the body diagonal of a cube of side 8 centered about the point $(4,4,4)$. These rotated bead packs are shown in the bottom row of figure 8.1, and their corresponding simulated internal magnetic field and gradient maps are shown in figure 8.4. The resultant $|g|-B_{z}^{i}$ correlations are shown in figure 8.5.

The $|g|-B_{z}^{i}$ correlations of the rotated bead packs show that rotating the bead packs has resulted in an greater amount of large magnitude gradients to be correlated with more positive field offsets. The closely packed bead pack in figure 8.5a still displays a greater quantity of gradient magnitude at large offsets compared to that of the loosely packed spheres in $b$, due to the fact that smaller 

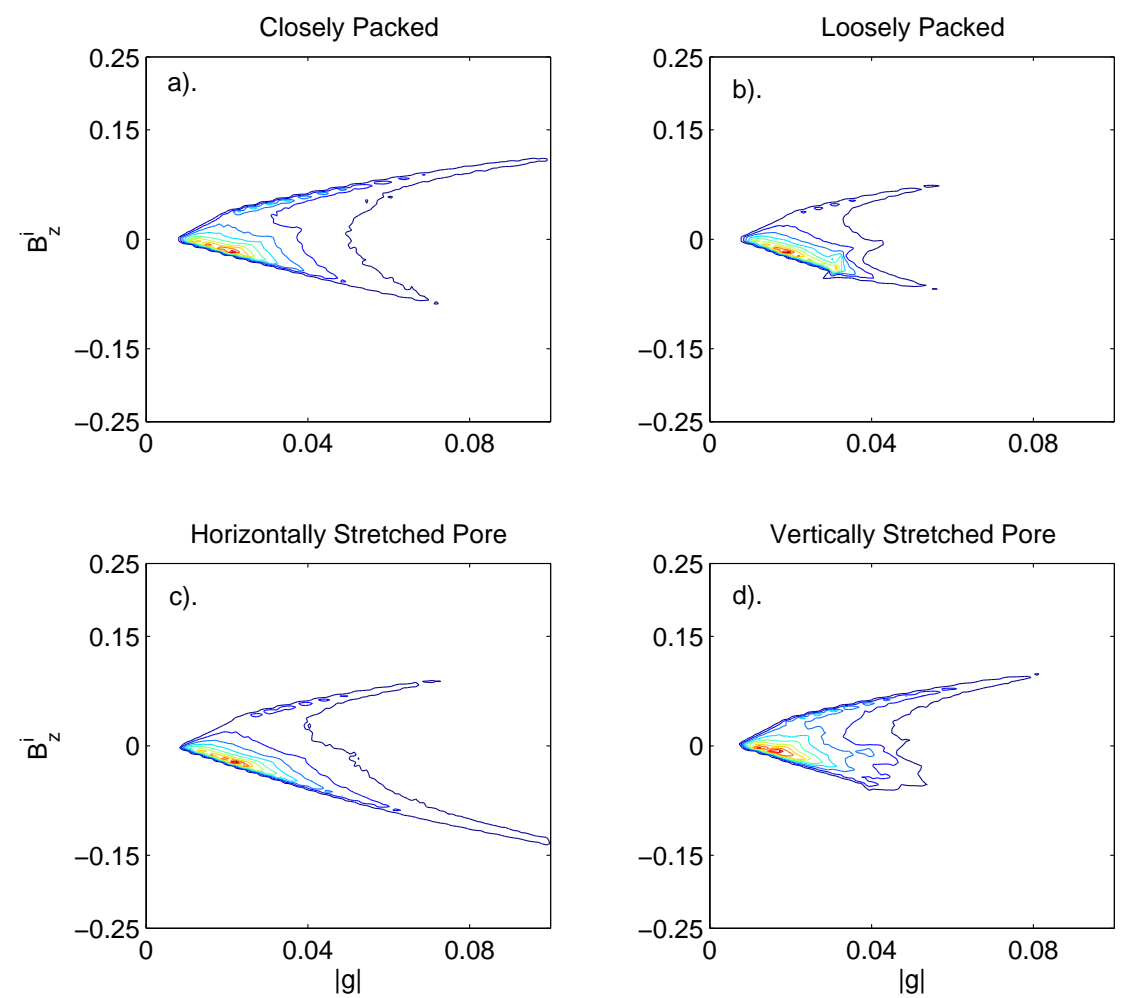

Figure 8.3: $|g|-B_{z}^{i}$ correlations for the beadpacks shown in the top row of figure 8.1. Contour levels range from 0.1 to 1.0 in intervals of 0.1 . Red indicates higher intensities.

pores will exhibit greater gradient magnitudes. We also see from $\mathrm{c}$ and $\mathrm{d}$ that we no longer see any evidence of the pore anisotropy after rotation of the bead packs. These figures indicate that while this method may not be sensitive to pore anisotropy unless the pores are aligned along the $x y-, x z^{-}$, or $y z$-plane, it is somewhat sensitive to pore size.

Moving on from our simplistic single pore models, we calculate the $|g|-B_{z}^{i}$ correlation of our simulated random bead pack as described in section 5.2. Comprised of many pores, this simulated bead pack will be a more realistic study of the interplay between magnetic field gradients and the field offset.

The maps of the resultant internal magnetic field and gradient magnitudes 

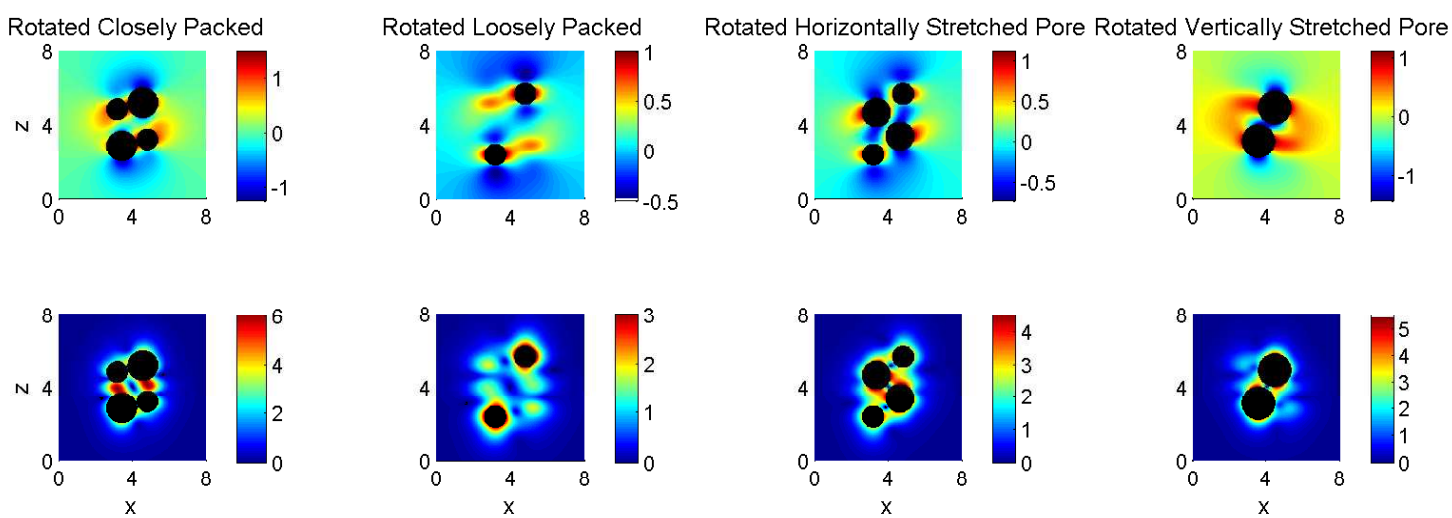

Figure 8.4: Top row: Simulated magnetic field maps for the bead packs shown in the bottom row of figure 8.1. Bottom row: The simulated magnetic gradient field magnitude for the same bead packs.

are shown in figure 8.6. Similar to the field and gradient maps of our single pore systems, we can see that areas of high gradient appear to occur most frequently near areas of large internal field offset, particularly at the surface of the spheres at the $z$-direction poles. When we take the correlation between the two, shown in figure 8.7, there are two things to notice. One, a diagonal wing appears which correlates large values of gradient magnitude with regions of large frequency offsets. This is to be expected due to the fact that from figure 8.6, box $B$, we see that regions of large frequency offset correspond to regions of high magnetic field gradient. Note that this spectrum does not have the large, pronounced diagonal wings that were present in the single pore correlations. This is probably in part due to the fact that this bead pack is comprised of reasonably close-packed monodisperse beads, therefore the contribution of all pores in the system will be similar in size and also to the effect of averaging many pore shapes and orientations.

Another trait of figure 8.7 is that there is a negative distortion wherein the largest magnitude gradients occur at regions of large negative field offset. This negative distortion corresponds to the skew in the 1D field spectrum which arises due to the more strongly negative region around the $z$-direction poles of the spheres which take up a smaller volume than the equatorial regions of 

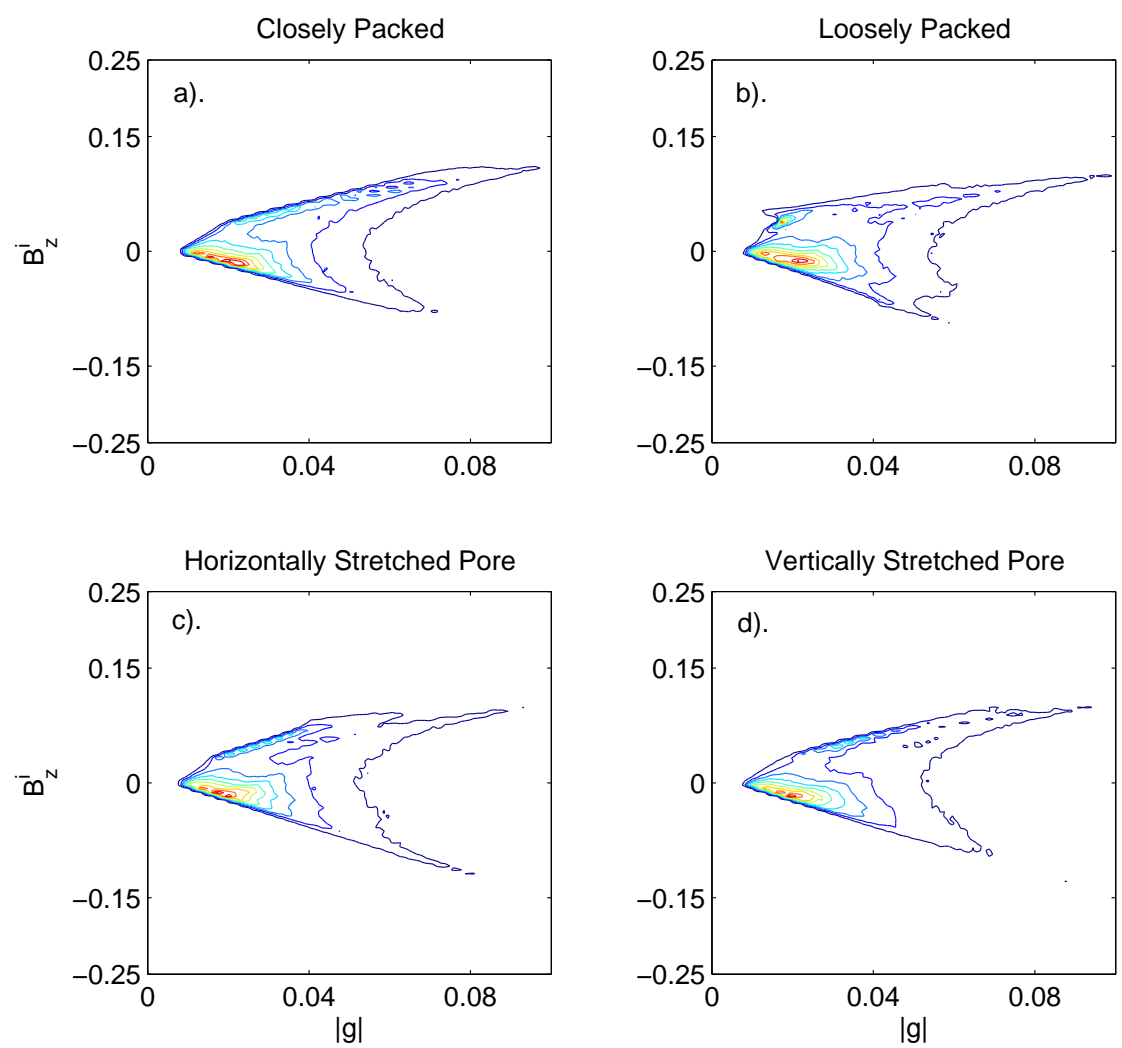

Figure 8.5: $|g|-B_{z}^{i}$ correlations for the beadpacks shown in the bottom row of figure 8.2. Contour levels range from 0.1 to 1.0 in intervals of 0.1 . Red indicates higher intensities.

positive field values. However, the average field is near zero with a value of -0.0191 . We suspect the slight negative value may be due to edge effects caused by bead ordering along the flat bottom of the bead pack.

\subsection{Summary of $|g|-B_{z}^{i}$ Correlation Spectra}

In general, the $|g|-B_{z}^{i}$ correlation will generate two different types of spectra. The first type is a peak which has diagonal wings that correlate larger field offsets with larger field gradients as shown schematically in figure 8.8a. The second 

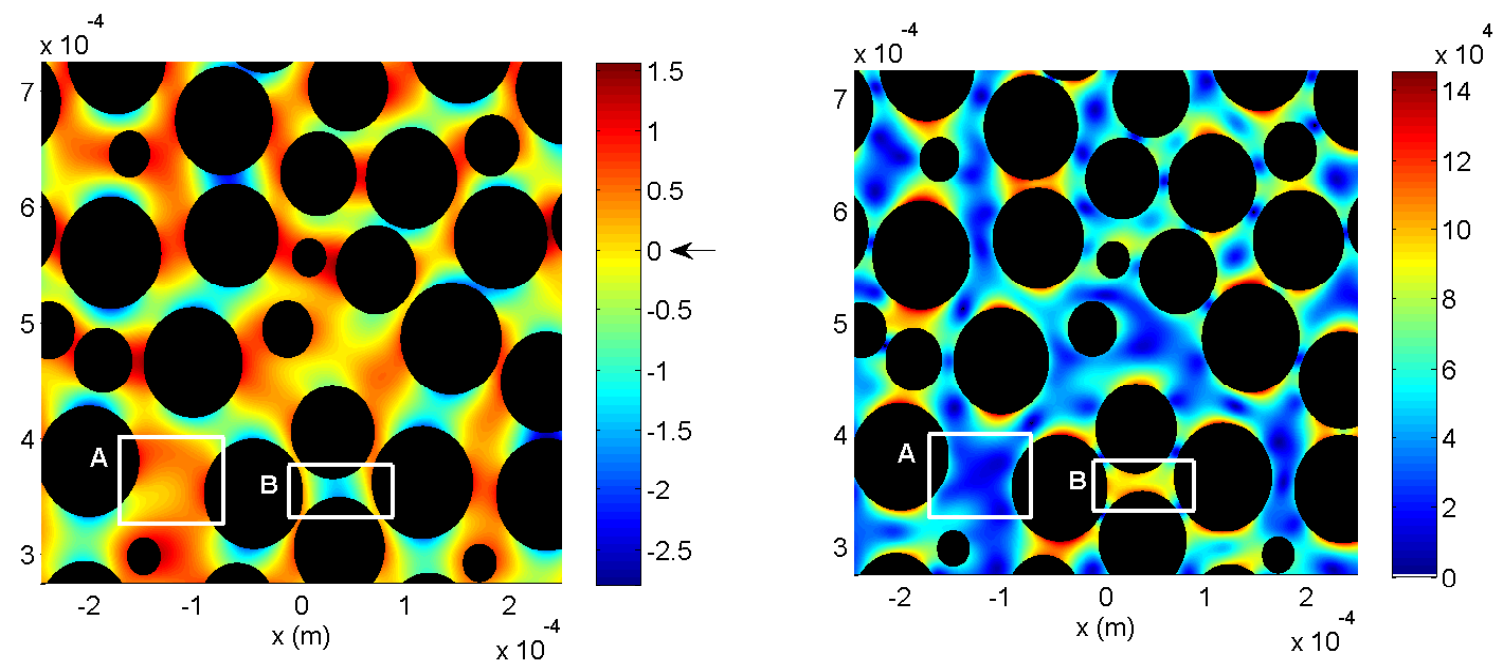

Figure 8.6: Left: The simulated internal magnetic field for the center $y$ slice of the cylindrical bead pack. Box A shows a low magnetic field offset corresponding to loosely packed beads. Box B shows a large magnetic field offset corresponding to tightly packed beads. Right: The simulated internal magnetic field gradient along the same slice of the left figure. Box A shows a region of loosely packed beads and thus a relatively low gradient magnitude. Box $\mathrm{B}$ shows a region of tightly packed beads resulting in a relatively high magnetic field gradient.

type of peak as shown in figure $8.8 \mathrm{~b}$, is a peak which rather than displaying diagonal wings, has horizontal wings, indicating that there is gradient growth without a change in field offset. Horizontal wings arise when $|g|$ is independent of field offset which occurs when there are smaller distances involved. Smaller distances are associated with smaller grains being present in the sample. Although the range of field offsets will remain the same, smaller grains mean that there will be much larger gradients as seen in figure 8.9. In this figure, two different size beads are displayed. The left column shows a bead of radius 0.5 units, and the right column shows a bead of radius 1 unit. The top row shows the simulated magnetic field generated by placing a $z$-oriented magnetic dipole at the center of each sphere. The field offset for both spheres ranges from approximately -2 to 1 frequency units. However, looking at the simulated gradient 


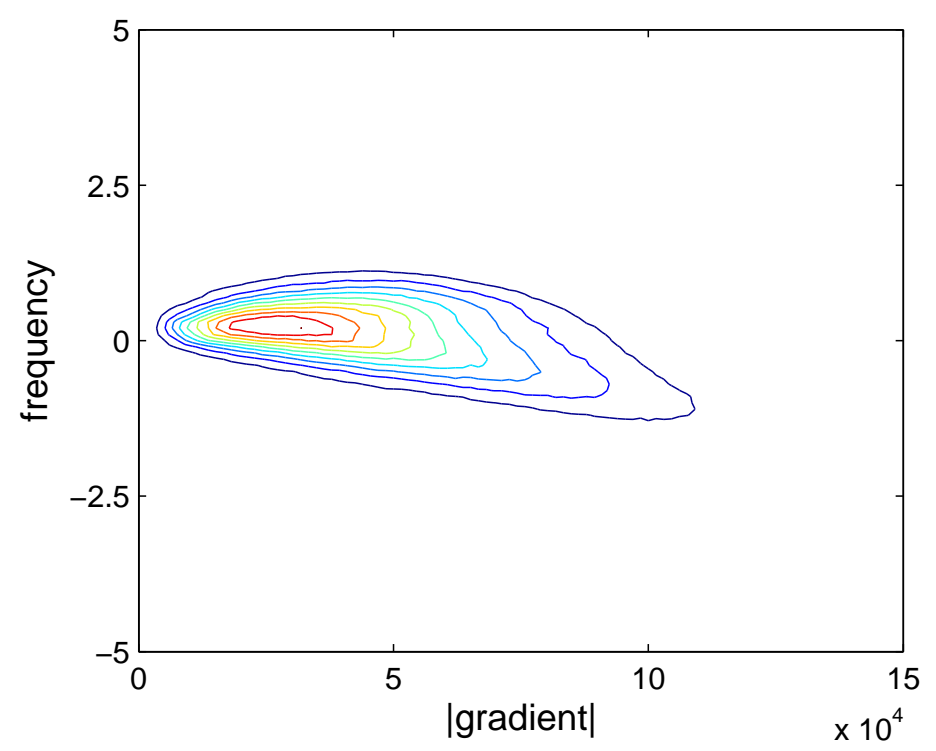

Figure 8.7: The simulated $|g|-B_{z}^{i}$ correlation. Both frequency and gradient units are dimensionless. Contour levels range from 0.1 to 1.0 in intervals of 0.1. Red indicates higher intensities.
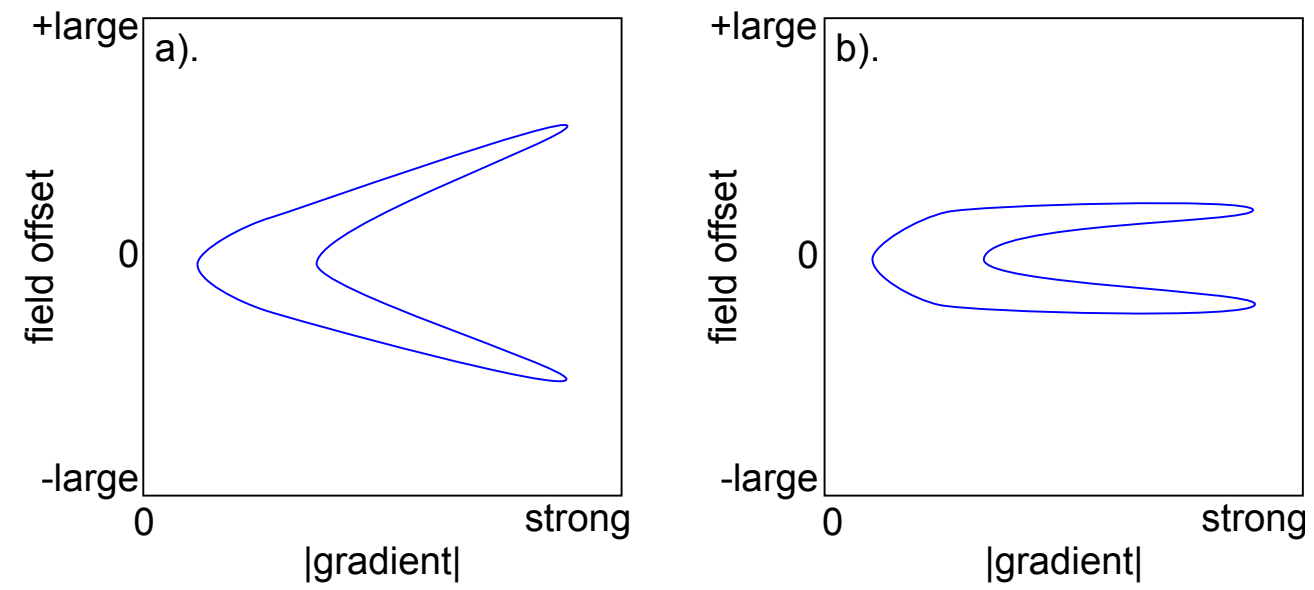

Figure 8.8: Schematic representations of a). The $|g|-B_{z}^{i}$ spectrum type with diagonal wings which correspond to large gradient magnitudes at large field offsets. b). The $|g|-B_{z}^{i}$ spectrum type with horizontal wings which correspond to small grain sizes. 
magnitudes for each sphere in the bottom row, we see the smaller sphere has a maximum gradient magnitude of about 12 , while the larger bead has a maximum gradient about half that at about 6 . Therefore, horizontal wings are indicative of large grain heterogeneity, where we have the presence of both large grains and groups of smaller grains.
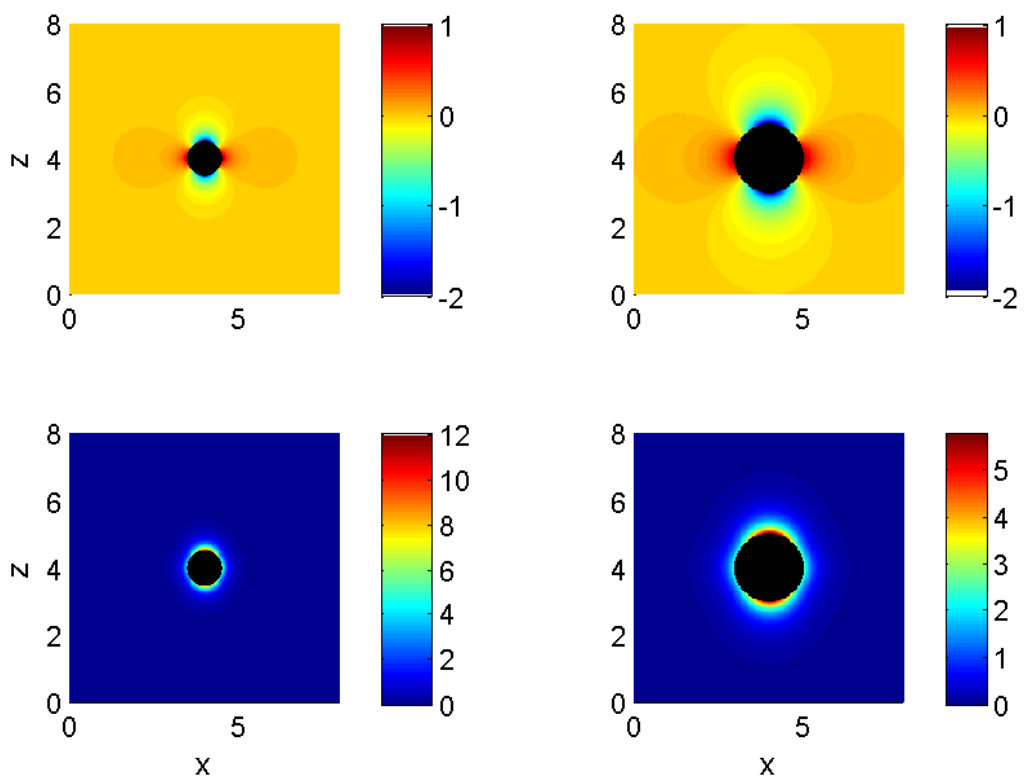

Figure 8.9: Left column: Field (top) and gradient (bottom) maps for a sphere of radius 0.5 units. Right column: As for right column but with a sphere of radius 1 unit. Note that while the range of field offsets are similar for both beads, the maximum gradient values are twice as large for the smaller bead than that of the larger bead.

From here, we move away from our simulation and move on to our $T_{2}-B_{z}^{i}$ experiment with varying $\tau$. 


\subsection{Pulse Sequence}

The pulse sequence used is shown in figure 8.10 and is a simple CPMG train as originally discussed in section 3.2.4. We start our pulse sequence with a $90^{\circ}$ pulse to bring our magnetization into the transverse plane. Following a time $\tau$, we apply a $180^{\circ}$ pulse, and wait another time $\tau$ and repeat our $180^{\circ}$ pulse $n$ times. We then acquire from the spin echo maximum to a time $t_{1}$ over $m$ points. The values of $\tau, m, n$, and $t_{1}$ depend on the sample being used. For a $100 \mu m$ monodisperse bead pack, $\tau$ ranges from $62.5 \mu \mathrm{s}$ to $1 \mathrm{~ms}$ with corresponding $n$ values ranging from 8196 and 512 for a total echo train time of $\sim 1 s$ for all $\tau$ values. We also acquire an $m$ value of 512 decay points in each echo. For a Bentheimer sandstone, $\tau$ values range from $125 \mu \mathrm{s}$ to $1 \mathrm{~ms}$ with corresponding $n$ values of 16384 and 512, for an echo train time of $\sim 4 s$ for all $\tau \mathrm{s}$, with an $m$ value of 256. We select $p$ echoes out of each echo train. For both samples $p=256$. This results in an $m \times p$ matrix with $m$ points of echo decay and $p$ echoes. By taking a Fourier transform along the echo decay dimension we acquire a spectrum of the broadened NMR frequency spectrum due to susceptibility differences between the solid matrix of our sample and its fluid filled pore space. The application of an inverse Laplace transform as described in section 2.9.2 along the CPMG dimension will allow us to obtain the $T_{2}$ distribution of our sample. This means we have a $2 \mathrm{D}$ data set that requires a combination of Fourier transform and inverse Laplace transform for analysis.

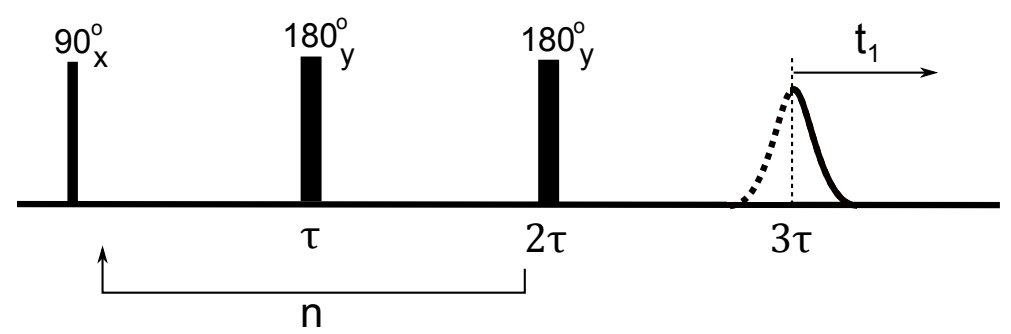

Figure 8.10: The CPMG pulse sequence used for $T_{2}-B_{z}^{i}$ correlation. Note we acquire from the echo maximum to a time $t_{1} . \tau$ is kept constant for all $n$. 


\subsection{Relaxation in the Inhomogeneous Magnetic Field}

The resulting magnetization decay from the pulse sequence of figure 8.10 will be a function of the inhomogeneous field and the transverse relaxation decay, $T_{2}$. Without diffusion, the magnetization due to $T_{2}$ relaxation is given by

$$
\frac{M\left(t_{T_{2}}\right)}{M_{0}}=\int P\left(T_{2}\right) \exp \left(-t_{T_{2}} / T_{2}\right) d T_{2}
$$

where $t_{T_{2}}$ is the time associated with $T_{2}$. The magnetization decay due only to diffusion through the inhomogeneous magnetic field is

$$
\frac{M\left(t_{T_{2}}\right)}{M_{0}}=\int P\left(g^{2}\right) \exp \left(-\gamma^{2} g^{2} \tau^{2} D t_{T_{2}} / 3\right) d g^{2}
$$

The total magnetization relaxation decay from diffusion through the inhomogeneous field is obtained by combining equations 8.1 and 8.2. We first define the relaxation time, $T_{2}^{\prime}$ which is a function of $g^{2}$

$$
\frac{1}{T_{2}^{\prime}\left(g^{2}\right)}=\frac{1}{T_{2}}+\gamma^{2} g^{2} \tau^{2} \frac{D}{3}
$$

Then the total magnetization relaxation decay from diffusion through the inhomogeneous field is given by

$$
\frac{M\left(t_{T_{2}}\right)}{M_{0}}=\int P\left(T_{2}^{\prime}\left(g^{2}\right)\right) \exp \left(-t_{T_{2}^{\prime}} \frac{1}{T_{2}^{\prime}}\right) d T_{2}^{\prime}\left(g^{2}\right)
$$

We also need to take into account the line broadening from the inhomogeneous field. We know the magnetization for a distribution of spins freely precessing in an external magnetic field is

$$
\frac{M\left(t_{\omega}\right)}{M_{0}}=\int P(\omega) \exp \left(-i \Delta \omega t_{\omega}\right) d \omega
$$

where $t_{\omega}$ is the time associated with the frequency. We can describe the magnetization following the pulse sequence as a combination of equations 8.4 and 8.5

$$
\begin{aligned}
\frac{M\left(t_{\omega}, t_{T_{2}^{\prime}}\right)}{M_{0}} & =\iint P\left(\omega, T_{2}^{\prime}\left(g^{2}\right)\right) \exp \left(-t_{T_{2}^{\prime}} \frac{1}{T_{2}^{\prime}}\right) \exp \left(-i \Delta \omega t_{\omega}\right) d \omega d T_{2}^{\prime}\left(g^{2}\right) \\
& +\epsilon\left(t_{\omega}, t_{T_{2}^{\prime}}\right)
\end{aligned}
$$


where $P\left(\omega, T_{2}^{\prime}\left(g^{2}\right)\right)$ is the joint probability density and $\epsilon\left(t_{\omega}, t_{T_{2}^{\prime}}\right)$ is the experimental noise. The joint probability density will be our correlation spectrum. In order to retrieve this value from equation 8.6, we need to use a Fourier transform followed by an inverse Laplace transform.

\subsection{Analysis of FT-ILT Data}

There are two main ways to analyze FT-ILT data, namely univariate and multivariate. Univariate processing, first described by Morris and Johnson [108], uses a simple 1D FT and then an iterative 1D ILT. This method is best used for samples which have a single species. Multivariate processing is first published by Stilbs [109] in which they use a global method called CORE-NMR for COmponent REsolved NMR spectroscopy. Unlike simply taking a Fourier transform and incrementally applying an Inverse Laplace, the CORE method uses the common echo attenuation for each component in the frequency spectrum to fit a global self-diffusion coefficient for each frequency spectrum component. In other words, the univariate method treats each signal as a separate decay, while the multivariate method fits the entire spectra simultaneously.

Since we are only measuring the NMR spectrum of distilled water in the pore space of a sample, we can use the univariate method rather than a multivariate method. To be sure that the univariate method will capture any overlapping peaks we may have in the projections along the frequency or $T_{2}^{\prime}$, we apply the univariate method to a simulated data matrix with a known test spectrum. This model spectrum first involves generating a test spectrum from which we can create a data matrix and then subsequently apply an FT and an iterative ILT to try to replicate the original test spectrum. This will involve 4 steps:

1. Create a 2D test spectrum, $T\left(T_{2}^{\prime}, \omega\right)$, using a combination of a Gaussian peak along the linear (frequency domain) axis, and a lognormal peak along the logarithmic (relaxation time domain) axis.

2. Apply a forward Laplace transform along the to $T\left(T_{2}^{\prime}, \omega\right)$ to obtain part of the data matrix $D\left(t_{T_{2}^{\prime}}, \omega\right)$. 
3. Apply an inverse Fourier transform to $D\left(t_{T_{2}^{\prime}}, \omega\right)$ to obtain the final data matrix, $D\left(t_{T_{2}^{\prime}}, t_{\omega}\right)$.

4. To generate the calculated spectrum, $S\left(T_{2}^{\prime}, \omega\right)$, to be compared with $T\left(T_{2}^{\prime}, f\right)$, apply an FT and then an iterative ILT to $D\left(t_{T_{2}^{\prime}}, \omega\right)$.

\subsubsection{Generating the Test Spectrum}

The test spectrum is meant to replicate the frequency- $T_{2}^{\prime}$ plots of the $T_{2}^{\prime}-B_{z}^{i}$ correlation experiment. Since frequency is typically displayed on a linear scale, and $T_{2}^{\prime}$ displayed on a logarithmic scale, we need a solution spectrum that has a linear dimension and a logarithmic dimension.

One of the concerns with taking a Fourier transform of the data and then doing a 1D inverse Laplace transform sequentially along the CPMG time domain is that correlation of information between the individual rows will be lost. In other words each individual row is treated independently. So for our test spectra, we use Gaussian peaks which span a number of frequencies and $T_{2}^{\prime}$ times to test if any information is lost between the rows when we analyze our data. That is, we will be seeking to establish whether the Gaussian shape of the spectrum is retained along the relaxation time dimension.

To make our peaks, we use a combination of a Gaussian peak along the linear axis (the frequency domain) which we designate as the $y$-axis, and a lognormal peak along the logarithmic $x$-axis (the transverse relaxation domain). The equation for a 1D non-normalized Gaussian is given by

$$
f(y)=\exp \left(\frac{-\left(y-y_{0}\right)^{2}}{2 \sigma_{y}^{2}}\right)
$$

where $y_{0}$ defines the center of the Gaussian and $\sigma_{y}^{2}$ is the variance of the function. The 1D lognormal function has a similar form to that of the Gaussian

$$
f(x)=\exp \left(\frac{-\left(\log (x)-\log \left(x_{0}\right)\right)^{2}}{2 \sigma_{x}^{2}}\right)
$$

where $x_{0}$ is the center of the distribution on a logarithmic scale and $\sigma_{x}^{2}$ is the variance of the function. 
The equation for the 2D lognormal-normal non-correlated distribution will be given by the product of equations 8.7 and 8.8 :

$$
f(x, y)=\exp \left(\frac{\left(y-y_{0}\right)^{2}}{2 \sigma_{y}^{2}}-\frac{\left(\log (x)-\log \left(x_{0}\right)\right)^{2}}{2 \sigma_{x}^{2}}\right) .
$$

This equation will give us a peak like that shown in figure 8.11a. To add multiple peaks, we simply sum together their associated functions.

To generate an ellipsoidal peak that is oriented along an angle to the $x$ - and $y$-axis, we add a correlation function to equation 8.9

$$
f(x, y)=\exp \left(\frac{\left(y-y_{0}\right)^{2}}{2 \sigma_{y}^{2}}-\frac{\left(\log (x)-\log \left(x_{0}\right)\right)^{2}}{2 \sigma_{x}^{2}}-\frac{b}{4} \frac{\left(\log (x)-\log \left(x_{0}\right)\right)\left(y-y_{0}\right)}{\sigma_{x}^{2} \sigma_{y}^{2}}\right)
$$

where the value $b / 4$ defines the eccentricity of the ellipse which must be a value between 0 and 1 . This equation will produce a peak such as that shown in figure 8.11b.
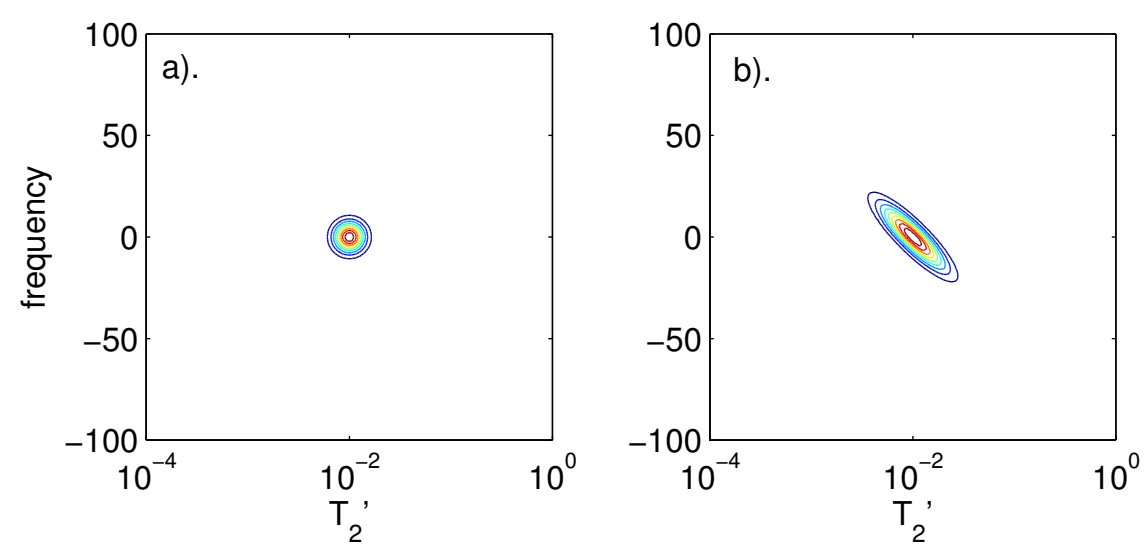

Figure 8.11: a). An example of a non-correlated Gaussian peak using equation 8.9. b). An example of a correlated skewed Gaussian peak using equation 8.10.

For our different test spectra, we use three spectra, two of which will result in the same projections along the $x$ - and $y$-axis to ensure that our analysis method 
is able to distinguish between the placement of the two peaks in the spectrum when the projections are the same. These two spectra are shown in figure 8.12a and $\mathrm{b}$. The third spectrum has a projection which includes overlapping peaks in both the $x$ - and $y$ - axis, as shown in figure 8.12c.
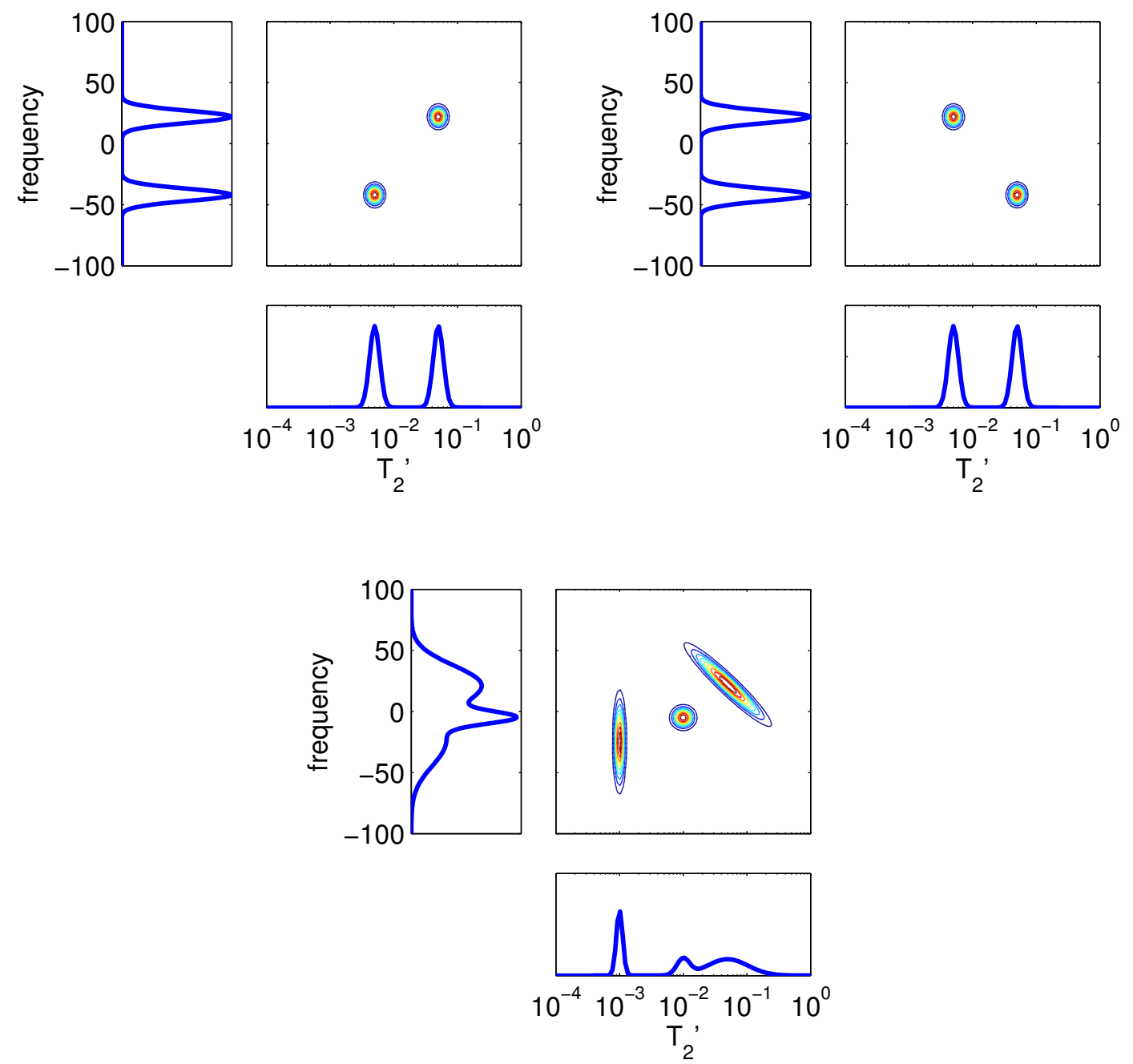

Figure 8.12: a). Two peaked solution spectrum with similar projections in the $x$ and $y$ directions. b). As for a). but in a different orientation. c). Test spectrum with overlapping peaks in the $\mathrm{x}$ and $\mathrm{y}$ projections. 


\subsubsection{Creating and Processing the Data Matrix from the Test Spectra}

Our test matrix, defined as $\mathbf{T}$, is an $m \times n$ matrix with $m$ points of linearly spaced frequency values and $n$ points of logarithmically spaced $T_{2}^{\prime}$ values. Our desired data matrix, $\mathrm{D}$ is defined by the matrix equation

$$
\mathbf{D}=\mathbf{F}^{-1} \mathbf{T}^{T} \mathbf{K}
$$

where $\mathbf{K}$ is the kernel function which is an $n \times p$ matrix of exponential decays $\exp \left(-t_{i} / T_{j}\right)$, where $p$ is the number of $t_{i}$, and $\mathbf{F}^{-\mathbf{1}}$ is the inverse Fourier transform of $\mathbf{T}^{T} \mathbf{K}$. D will be a matrix of size $m \times p$.

Now that we have $\mathbf{D}$ we proceed with the combined Fourier-inverse Laplace transform to see if we can reproduce the sample spectra of figure 8.12. First we take the magnitude of the Fourier transform along $m$ and sequentially apply the inverse Laplace transform to each row in the Fourier transformed matrix. The same regularization parameter, $\alpha$ is used for each inverse Laplace transform.

The results of this processing are shown in figure 8.13, where the top row shows the starting test spectra and the bottom row shows the results of the processed data matrix. The calculated results show slight broadening with respect to $T_{2}^{\prime}$ compared to the solution spectra, and the calculated spectrum of the rightmost column shows a loss of signal in the shortest $T_{2}^{\prime}$ peak. Overall, these spectra show that information is not lost using this processing method and that it will be appropriate to use for our $T_{2}^{\prime}-B_{z}^{i}$ correlation data. It is important to note that in the rightmost column of spectra the shortest $T_{2}^{\prime}$ peaks in both the test and calculated spectra appear to have two different intensities due to slight broadening along the $T_{2}^{\prime}$ domain for the calculated spectrum. When both peaks are integrated with respect to frequency and $T_{2}^{\prime}$, they are found to have equivalent total intensities. 

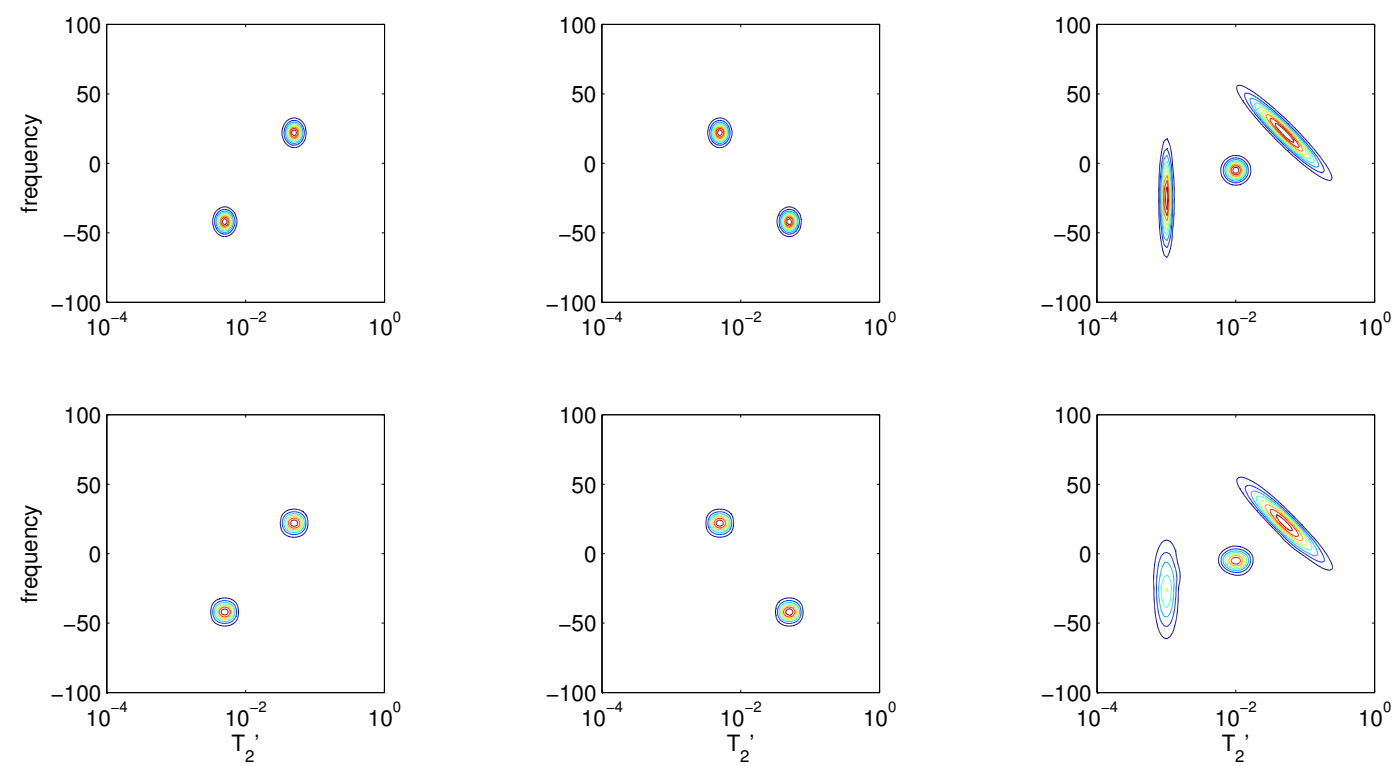

Figure 8.13: Top row: Test spectra from figure 8.12. Bottom row: Calculated spectra after processing by the FT-sequential ILT method. Note that for the rightmost column, the intensities for all peaks are the same. The apparent decrease in intensity for the short $T_{2}^{\prime}$ peak in the calculated spectrum is due to the slight broadening along the $T_{2}^{\prime}$ domain. 


\section{7 $T_{2}-B_{z}^{i}$ Correlation for a Monodisperse Glass Bead Pack}

Using the same $100 \mu \mathrm{m}$ monodisperse bead pack as described in chapter 6 , we perform a set of $9 T_{2}^{\prime}-B_{z}^{i}$ correlation experiments each with a different value of $\tau$ ranging from a minimum of $62.5 \mu \mathrm{s}$ to a maximum of $1 \mathrm{~ms}$. A spectral width of $100 \mathrm{kHz}$ is used and 512 points are acquired after the maximum of the last echo in the CPMG train. The values of $n$ range from 512 to 8192 with 256 echoes selected from each CPMG train. We select only the even echoes so as to eliminate the odd-even echo effect. All experiments are performed at a constant temperature of $25^{\circ} \mathrm{C}$. These results are shown in figure 8.14.

From figure 8.14, we can see that for $\tau$ values from $500 \mu$ s and greater, there is some slight structure on the short $T_{2}^{\prime}$ side of the peak which we would expect to arise from the increasing role of internal field gradients as the echo time $\tau$ is increased. What we immediately notice in the case of $\tau=1 \mathrm{~ms}$ is the appearance of horizontal "wings". Such wings, as we have argued earlier, are indicative of grain size heterogeneity. Why then do we observe such effects in a monodisperse bead pack? While our bead pack only has one bead size and two susceptibilities arising from the water and glass, heterogeneities can arise from the randomness in packing. For example, more loosely packed beads will have a smaller magnetic field gradient since the length scale over which changes in field occur will be larger. Such a region is shown in the right plot in figure 8.6, box A. By contrast, more densely packed areas (as shown by box B), will experience the same field change but over a shorter distance, leading to a larger value of field gradient. Of course, inspection of the corresponding field map (right plot in figure 8.6), also shows that more loosely packed spheres (box A) also have a smaller average field offset and more densely packed spheres (box B), have a larger average field offset. Therefore, in a heterogeneous sample we might expect that lower gradients correspond with smaller field offsets. This is the basis of the "diagonal wings" argument, and indeed, such features are apparent in the broadly triangular map obtained in the simulation itself, figure 8.7. But closer inspection of this figure shows that at the highest gradients, 

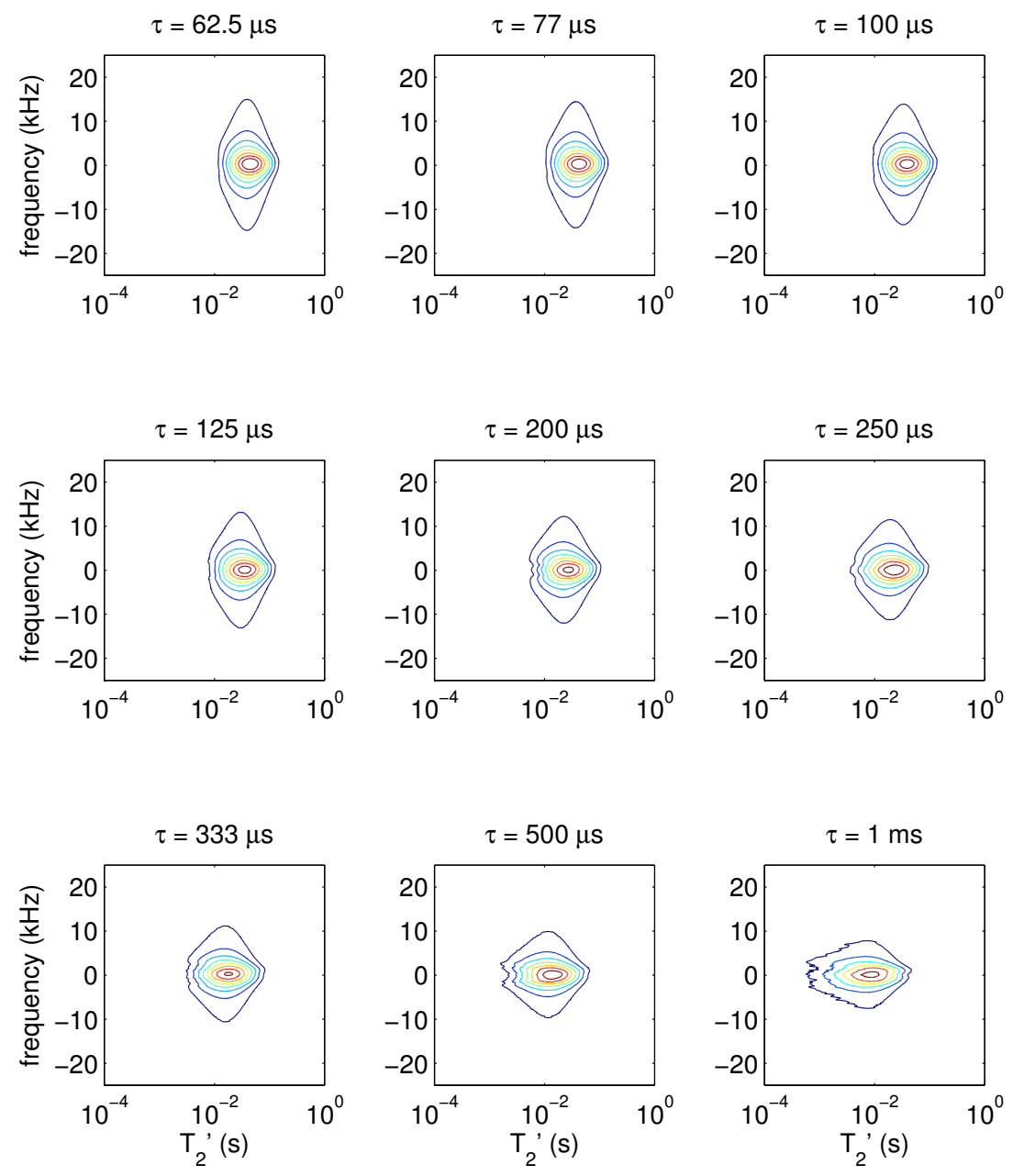

Figure 8.14: The $\mathrm{T}_{2}^{\prime}$-B correlation for a monodisperse $100 \mu m$ glass bead pack. Bandwidth shown is $50 \mathrm{kHz}$. $\tau$ values range from $62.5 \mu \mathrm{s}$ to $1 \mathrm{~ms}$. Note that at the longest $\tau$ value internal field gradients have more significant influence on $T_{2}^{\prime}$ contributing to extended peak structure at the shorter $T_{2}^{\prime}$ values. Contour levels range from 0.1 to 1.0 in intervals of 0.1 . Red indicates higher intensities. 
a horizontal "flattening" occurs. This is consistent with the "similar fields but different length scales" picture. However the effect is subtle, both in our simulation and in the bead pack experiments. In heterogeneous materials much larger variations in gradient will arise through changes in characteristic local length scales, and much more pronounced horizontal wings may arise. Thus we see the $T_{2}^{\prime}-B_{z}^{i}$ correlation experiment as potentially providing a signature for the degree of grain size heterogeneity. This we test in an experiment on a rock sample as described in the next section.

Before leaving our discussion of the monodisperse bead pack, we need to address the differences apparent in the simulation shown in figure 8.7 and the experimental results shown in figure 8.14. First, note that they are plotted in a different way, as $|g|-B_{z}^{i}$ maps in the former and $T_{2}^{\prime}-B_{z}^{i}$ maps in the latter. Still as $\tau$ becomes large we would expect the $T_{2}^{\prime}-B_{z}^{i}$ map to be indicative of $|g|-B_{z}^{i}$, albeit with horizontal inversion, i.e. shorter $T_{2}^{\prime}$ indicating larger $|g|$. It may be that recalculating the $T_{2}^{\prime}-B_{z}^{i}$ maps as $|g|-B_{z}^{i}$ maps might bring about a greater correspondence. This we have not attempted here due to time constraints, but such analysis could be the basis of future work. However, we have also to acknowledge that the simulated bead pack and the actual experimental bead pack are different, and indeed have different porosities. Hence we would not necessarily expect identical local gradient patterns, especially since these are derived from a spatial derivative which may be highly sensitive to structure.

The important point we wish to establish is that these simple $T_{2}^{\prime}-B_{z}^{i}$ experiments can provide a signature for length scale heterogeneity. That this is so becomes very clear when we apply the experiment to a real rock sample.

\section{8 $T_{2}^{\prime}-B_{z}^{i}$ Correlation for a Bentheimer Sandstone Core}

To observe a greater correlation between $T_{2}^{\prime}$ and $\Delta B_{i}^{z}$, we need a sample with larger heterogeneity than a monodisperse glass bead pack. We select a Bentheimer sandstone core of diameter $12.3 \mathrm{~mm}$ and length $40 \mathrm{~mm}$ and saturate it with a $3 \%$ weight $\mathrm{KCl}$ brine solution. In preliminary experiments using a $5 \mathrm{~mm}$ diameter core, the long duration of our frequency resolved CPMG experiments 
caused evaporation of the brine out of the core which then condensed on the inside of our sample tube resulting in a change in resonance frequency and CPMG decay as the experiment progressed even with temperature control. To eliminate this evaporation we sealed a new core in Perspex acrylic. Xray-CT has been used to create a tomogram of a Bentheimer core (shown in figure 8.15) and has also been used to determine the material components of the sandstone [110]. Bentheimer is comprised of equal parts of the mineral kaolinite $\left(\mathrm{Al}_{2}\left[\mathrm{Si}_{2} \mathrm{O}_{5}\right](\mathrm{OH})_{4}\right)$ and diamagnetic clay regions. The placement of these clay regions within the core determines the total heterogeneity of the sample.

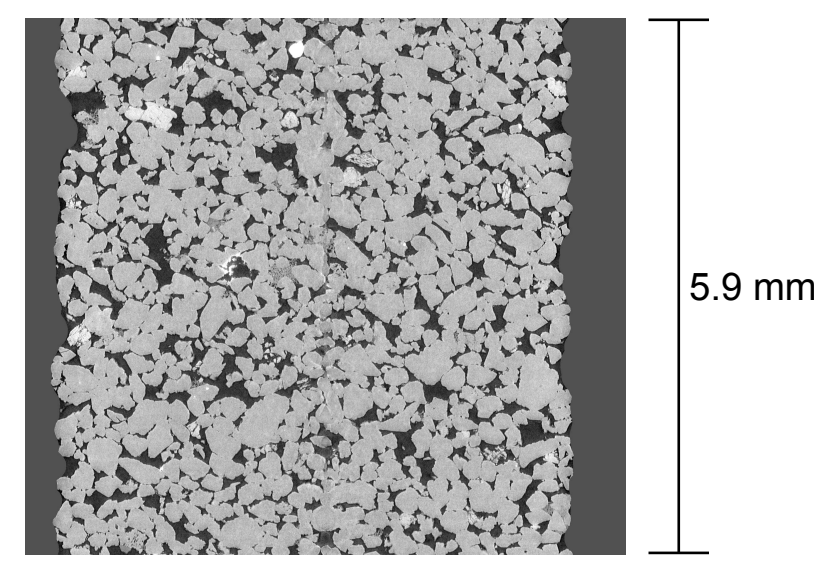

Figure 8.15: X-ray tomogram of a $5 \mathrm{~mm}$ diameter Bentheimer sandstone. The resolution per voxel is $(2.8 \mu \mathrm{m})^{3}$.

For our Bentheimer experiments, we use $\tau$ times ranging from $125 \mu \mathrm{s}$ to $1 \mathrm{~ms}$. Additional experiments of 2 and $4 \mathrm{~ms}$ were also performed, but the data quality was very poor due to the large echo attenuation. All experiments are performed at a constant temperature of $25^{\circ} \mathrm{C}$. The full width half maximum (FWHM) of the Betheimer is approximately $1.7 \mathrm{kHz}$, so we used a spectral width of $20 \mathrm{kHz}$ in our experiment. Our $n$ values range from 16384 to 512 with 256 echoes selected from each CPMG train.

The results are shown in figure 8.16 and again, similar to the $100 \mu m$ bead pack, there is structure at larger values of $\tau$, however, unlike the bead pack, the structure for Bentheimer is more pronounced, and can be seen as early as $250 \mu s$, indicating larger heterogeneity in the rock core. This heterogeneity can 
arise from different materials present in the core, such as paramagnetic grains or different pore sizes. There is a much sharper point at long $T_{2}^{\prime}$ values compared to the bead pack, also indicating larger heterogeneity.
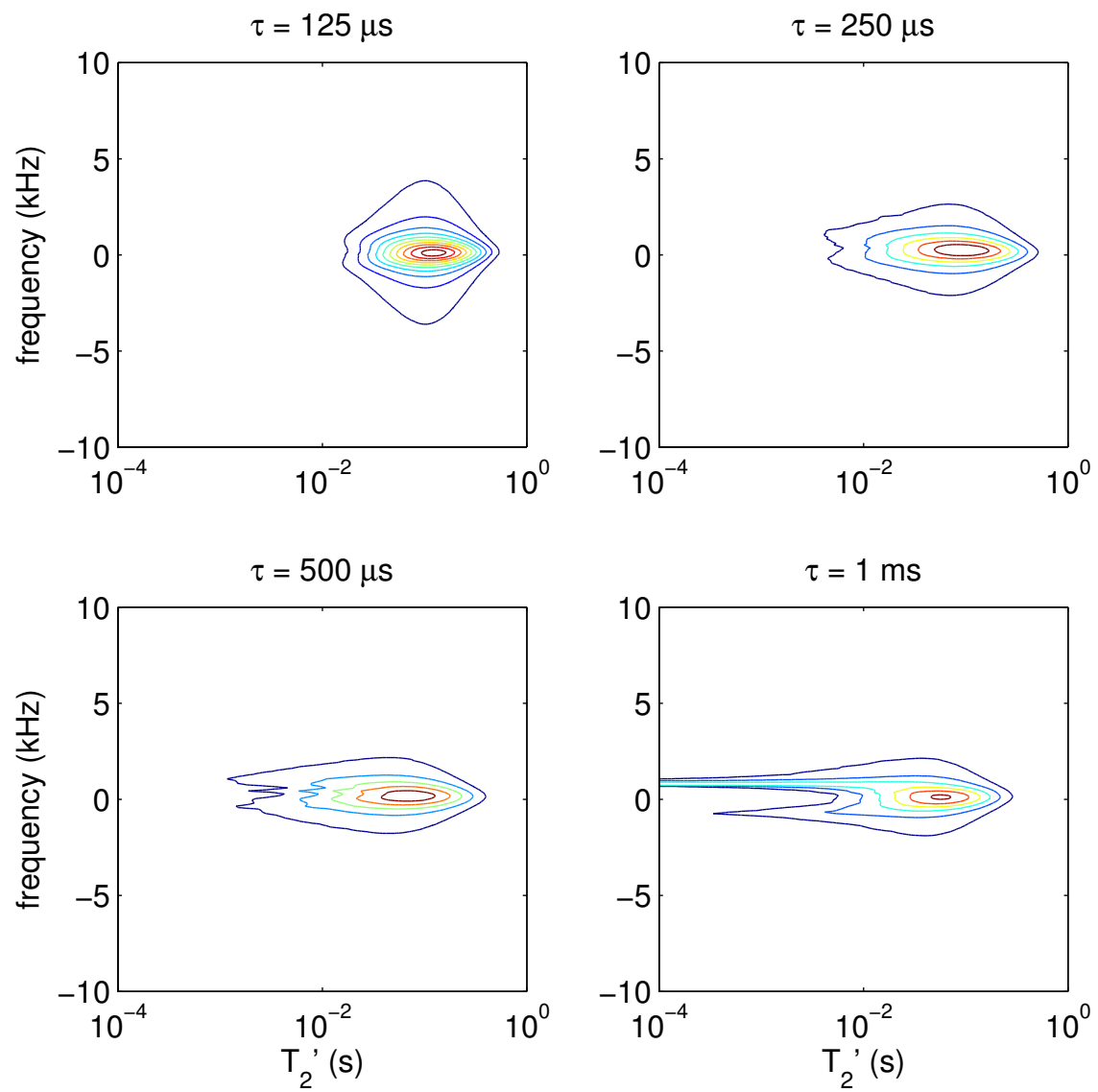

Figure 8.16: The $T_{2}^{\prime}$-B correlation for a Bentheimer. Bandwidth shown is $20 \mathrm{kHz} . \tau$ values range from $125 \mu \mathrm{s}$ to $1 \mathrm{~ms}$. Contour levels range from 0.1 to $1.0 \mathrm{in}$ intervals of 0.1 . Red indicates higher intensities.

From figures 8.14 and 8.16 , it is clear that this experiment can be used to indicate pore and grain heterogeneities in a sample. In rock the lobes of the inhomogeneous field spectrum are much more pronounced compared to those of the bead pack due to the different reasons for heterogeneities in the samples. In both samples, heterogeneities are caused by packing and pore size, whereas 
the rock, unlike the bead pack, is also made of different materials, and therefore can have different regions of susceptibility differences which in turn can cause larger internal field changes which will arise in larger field gradient values. Also note that the wings in the correlation remain at the same offset, indicating a large grain and pore heterogeneity since in general, the shape of the internal magnetic field remains the same independent of pore size, however, smaller pores will have larger gradients. Therefore, a growth in gradient intensity along a constant magnetic field offset indicates strong pore and grain heterogeneity.

\subsection{Simulating the $T_{2}-B_{z}^{i}$ Correlation in the Bead Pack}

The addition of relaxation to our simulation was discussed in depth in Chapter 5, section 5.7. However, it is important to note that our simulation is rather simplistic and treats relaxation as a function of proximity of tracer to bead. Namely, if a tracer reflects off a bead, there is a small probability $k$ that a tracer will "relax" and no longer contribute to the simulation. Since the internal magnetic field is only calculated as a function of position in the pore space before and after diffusion, we do not directly simulate the echo shape, therefore we will be unable to exactly simulate the experiment's dependence on $\tau$. However, we can simulate an overall correlation between $T_{2}$ and $B_{z}^{i}$, and acquire an exponential decay which can be inverted using the ILT to generate a 1D $T_{2}$ decay.

It is important to note that in this section we refer specifically to the $T_{2}$ relaxation caused by surface relaxation, and not the $T_{2}^{\prime}$ relaxation which is also a function of field gradient. This is due to the method used to simulate relaxation as described in section 5.7 where we calculate our simulated $T_{2}$ from a constant probability $k$ that a tracer will disappear if encounters a sphere, and we do not include relaxation due to diffusion through an inhomogeneous field.

Note that, our simulated $T_{2}$ distribution will look different from the $T_{2}^{\prime}$ distribution as measured in the experiment except at the time $\tau \rightarrow 0$, where $T_{2}^{\prime}\left(g^{2}\right) \rightarrow$ $T_{2}$. The $k$ value chosen is 0.05 , using the biexponential CPMG echo train fit as outlined in section 5.7. One way to determine the simulated $T_{2}$ distribution is by taking an ILT of the simulated echo amplitude decay. This decay can be found 
by cumulatively summing over the number of tracers that have not relaxed after each diffusion step. The echo amplitude decay for our simulation is shown in left figure in figure 8.17 with the subsequent $T_{2}$ distribution shown in the right figure in figure 8.17. As expected, comparing this distribution directly to experiment shows that the simulated $T_{2}$ distribution is slightly more broad and has a longer average $T_{2}$ time than that of the experimental distribution. Recall that the chosen $k$ value was based on the Brownstein-Tarr spherical pore calculation. It is not surprising that in the case of a bead pack, where the pores are far from spherical, there will be an incompatibility of the calculated $T_{2}$ distribution and the biexponential approximation to the CPMG echo decay, used to fit for $k$. Since the work here primarily concerns the shape of the 2D distribution, we can live with the mean $T_{2}$ offset, allowing that the overall spread in simulated $T_{2}$ values approximately matches the experimental data.
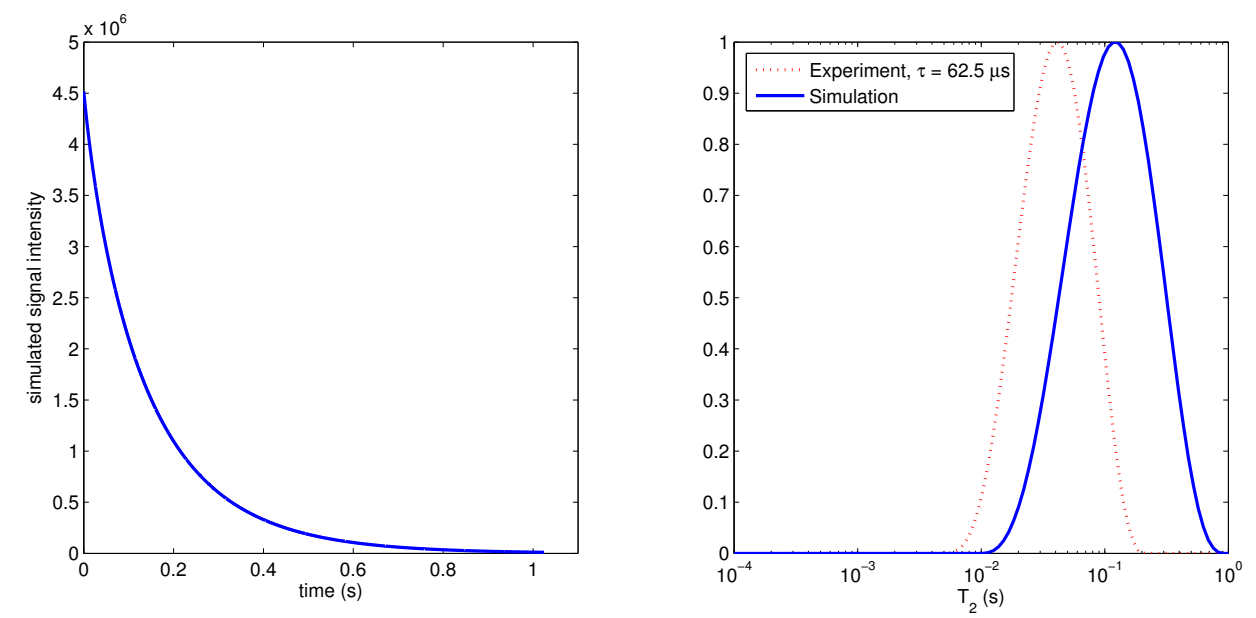

Figure 8.17: Left: The simulated echo amplitude decay. Right: The $\mathrm{T}_{2}$ distribution of the simulated echo decay from left is shown by the solid blue line. The $\mathrm{T}_{2}$ distribution for the monodisperse bead pack with a $\tau$ of $62.5 \mu \mathrm{s}$ is shown by the dotted red line. The value of $\tau$ is sufficiently short that $T_{2}^{\prime}\left(g^{2}\right) \leftarrow T_{2}$, as indicated by the $\tau$ independence of the $T_{2}^{\prime}$ distribution for $\tau<500 \mu s$.

While this method of determining the $T_{2}$ distribution is straightforward, it does not give us any information about the internal field for a particular relaxation time, therefore we must use another method. The $T_{2}-B_{z}^{i}$ correlation is 
simulated by assigning a $T_{2}$ time for each relaxed tracer as $T_{2}=j \Delta / N$ where $j$ is the number of diffusion steps a tracer has completed, $\Delta$ is the total diffusion time for the entire simulation, 1.0245 seconds, and $N$ is the maximum number of diffusion steps, which is 8196 for this simulation. Therefore, for each relaxed tracer, we have both a $T_{2}$ time, and the tracer's position information from which we can calculate the value of the internal magnetic field at the point where the tracer relaxed. With this information we can generate a $T_{2}-B_{z}^{i}$ correlation map, shown in figure 8.18.

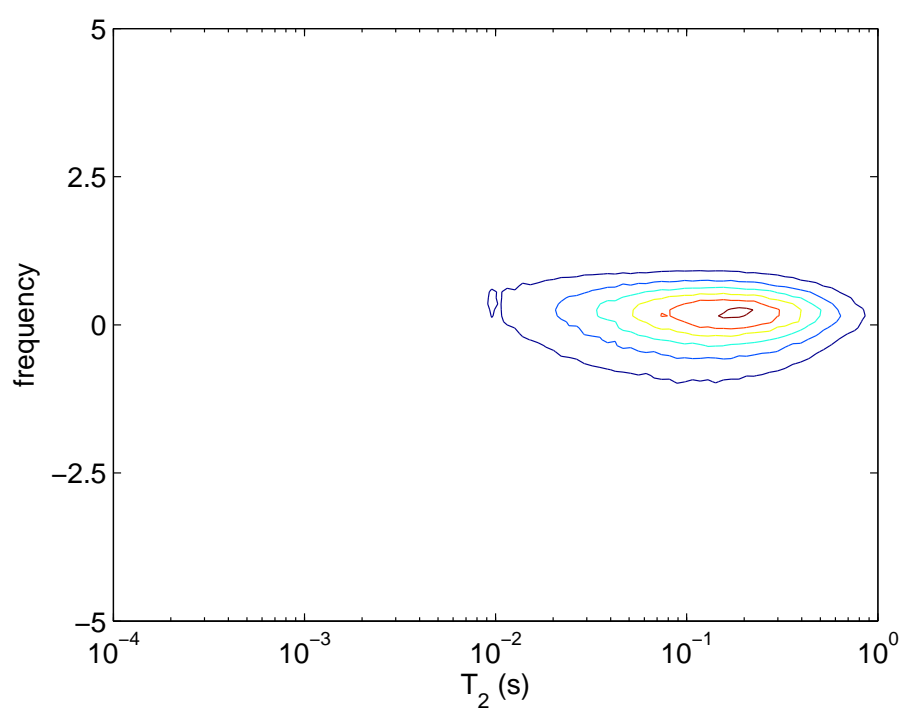

Figure 8.18: The simulated $\mathrm{T}_{2}-B_{z}^{i}$ correlation. Frequency units are dimensionless. Contour levels range from 0.1 to 1.0 in intervals of 0.1 . Red indicates higher intensities.

The most obvious feature of figure 8.18 is that we do not see any strong correlations between $T_{2}$ relaxation and field offset. This is due to the fact that the $T_{2}$ in our simulation is driven solely by surface relaxation and does not take into account the local magnetic field gradient. Again, we have room for improvement in the relaxation aspect of the simulation. Adding relaxation due to local field gradients would indeed produce a correlation with likely a triangular shape and a correlation between short $T_{2}$ times and large offset as relaxation would occur not only on the surface of the sphere as is currently being simulated, but also as 
a function of local gradient magnitude. This is a possibility for future work.

\subsection{Conclusions}

In this chapter we examined the correlation between echo-time dependent transverse relaxation, $T_{2}^{\prime}$, and the internal magnetic field, $B_{z}^{i}$, by using a frequency resolved CPMG train on a monodisperse glass bead pack and a core of Bentheimer sandstone. From our $|g|-B_{z}^{i}$ simulations, we find that two types of "wings" appear, either horizontal, which can be indicative of grain size heterogeneity, with horizontal wings indicating the presence of differing size grains, or diagonal, which is indicative of a wide range of local field strengths. We find in our monodisperse bead pack correlations hints of horizontal wings at long $\tau$ times which correspond to a strong influence of internal gradients on $T_{2}^{\prime}$. While horizontal wings are indicative of grain size heterogeneity, pore heterogeneities can appear due to the randomness in packing. The $T_{2}^{\prime}-B_{z}^{i}$ correlations of the Bentheimer sandstone have very clear horizontal wings at long $\tau$ times which we expect not only due to the smaller grains, but the greater range of grain sizes and the presence of multiple magnetic susceptibilities.

We also simulate $T_{2}-B_{z}^{i}$ correlations, however, due to the simplicity of the implementation of $T_{2}$ relaxation in the simulation, namely the fact it is dependent on proximity to sphere surface and does not include an internal gradient dependence, we do not see a strong correlation between $T_{2}$ and $B_{z}^{i}$. However, in future work, expanding the simulation to include relaxation due to local field strength should allow us to accurately simulate the experiment's dependence on $\tau$. Future experimental work may also include using rock cores of different heterogeneities, directly calculating the $|g|-B_{z}^{i}$ correlations, and simulating the Bentheimer rock core used in this chapter. Christoph Arns plans on creating this simulation in the near future. 


\section{Chapter 9}

\section{Summary and Future Work}

It is well known that the magnetic susceptibility difference between the solid matrix and fluid-filled void space of a porous medium results in an inhomogeneous internal magnetic field in the pore space when a sample is exposed to an external magnetic field, such as that which exists in an NMR machine. These inhomogeneities will cause line broadening in the 1D NMR spectrum and can affect the measurements of various properties such as relaxation and diffusion. In this thesis, we seek to exploit the presence of this inhomogeneous internal field to glean information about the pore space of the system. To accomplish this, we purposefully use samples which, upon exposure to an external magnetic field, will have large internal magnetic fields such as glass beads imbibed with water and brine-saturated sandstone. We first develop a program to simulate a bead pack with an inhomogeneous magnetic field and allow tracers to diffuse through the pore space via a random walk model. From there we utilize multi-dimensional NMR exchange methods which can allow us to separate the susceptibility induced broadening from the broadening caused by diffusion through the pore space. Later we use a 2D correlation method to correlate the field offset with transverse relaxation.

The most time-consuming work of this thesis was by far the simulation project. This simulation initially started as tracers generated along a mesh in the pore space of a cube centered in the cylindrical bead pack. These tracers diffused as a random walk along the $x y z$-axis. The cube was made small enough so 
that no tracers would encounter the edge of the cylindrical bead pack, and if a bead was encountered during the random walk, the tracer would bounce back to its previous step position along the $x y z$-axis. The internal magnetic field was calculated by placing a $z$-oriented magnetic dipole at the center of each sphere. While sufficient for simulating simple experiments, this program was not robust enough to accurately represent realistic $Z$-displacements which would manifest as propagators with oscillations. We rebuilt the diffusion stepping portion of the simulation from the ground up. First, to replicate more realistic diffusion, the random walk was changed to a walk along a random direction. Secondly, the basic reflection off a bead was changed from the simple bounce back to the previous tracer point, to a more realistic angled reflection off the sphere surface. Thirdly, we remove the mesh-based tracer generation and incorporate an algorithm which generates the tracers randomly in the pore space. Lastly, we no longer use a starting cube centered in the bead cylinder but rather define cylindrical boundaries just inside the bead pack which define a reflection boundary for any tracers which encounter them during diffusion.

For our first experiment, we used a two-dimensional exchange method to separate the line width broadening due to magnetic susceptibility differences from the line width broadening due to diffusion through the inhomogeneous internal magnetic field of the pore space. We found that by quantifying the offdiagonal intensity growth with $\tau_{m}$ as an average of two points on either side of the off-diagonal plotted with respect to mixing time results in a biexponential curve from which we can obtain a characteristic time and thus a characteristic length of the pore system. We use two different samples of monodisperse water filled glass bead packs at two different magnetic field strengths. We find this biexponential growth occurs at both magnetic field strengths and in bead samples with diameters as small as $10 \mu \mathrm{m}$, although since the mean displacement during the frequency encoding time at this size bead is comparable to a large fraction of the pore space, we do not put very much weight in the pore characterization results from this particular sample. However, results from the larger $100 \mu \mathrm{m}$ diameter bead pack look promising. Our simulated results also mesh well with experimental results.

Calculating a characteristic time based on temporal information alone while 
possible, is not precise, due to the fact the characteristic length we calculate is indirectly based on time and not directly based on spatial information. So for our next experiment we add a spatial dimension to the frequency exchange experiment in the form of a propagator. What this propagator allowed us to do is select 2D spectra based on the $Z$-displacement of the spins in the pore space of the system. From the propagators we are able to obtain the effective diffusion of the system and derive a theory which moves away from the simplistic biexponential fit of the previous chapter and directly calculates a characteristic length using a combination of the $Z$-displacement, mixing time, and effective diffusion. It is in this chapter where the simulation really shines. We're able to simulate the propagator dimension, and the spatially-resolved 2D exchange spectra. Again we find our simulation agrees well with experiment.

Finally, we move away from exchange experiments and correlate transverse relaxation with the inhomogeneous internal field. We find that as we increase the $\tau$ time between $180^{\circ}$ pulses, correlation between short $T_{2}$ times and large magnetic field offsets occurs. This correlation, while subtle in the $100 \mu \mathrm{m}$ bead pack, becomes more apparent when we use a brine-filled Bentheimer sandstone core. This increase in correlation indicates that the transverse relaxation and internal field correlation may be an indicator of sample heterogeneity. We correlate the internal magnetic field with the transverse relaxation, $T_{2}$, however, it is important to note we cannot directly simulate the echo-time dependent transverse relaxation, $T_{2}^{\prime}$ due to the nature of the software currently being unable to mimic relaxation which is dependent on local magnetic field gradients.

\subsection{Future Work}

While the work in this thesis comprises a good body of work which covers using the susceptibility induced inhomogeneous internal magnetic field to obtain information about the pore space of a sample, there is still more work which can be done.

One project could be on the continuing improvement of the simulation. In particular, work could be done with making a more realistic simulated trans- 
verse relaxation which is dependent on the local field gradient rather than solely on the proximity to bead. This would allow for a proper direct simulation of the $T_{2}-B_{z}^{i}$ correlations of the previous chapter.

Another project could involve using more realistic samples for both the propagator resolved and non-propagator resolved frequency exchange. While the glass bead samples are a nice model system, it would be interesting to see how the complexities of rock core samples would manifest themselves in the 2D spectra, particularly the spatially-resolved spectra.

As for the correlation experiment, a spatial dimension could be added to the $T_{2}-B_{z}^{i}$ correlation experiment which could give information on whether or not correlation seen in this experiment is dependent on $Z$-displacement. Along the same vein, one could add a three dimensional propagator to either the correlation experiment or the exchange experiment, but this may be limited by experimental time, as a three dimensional propagator resolved experiment may take a few days to run.

\subsection{Conclusion}

In conclusion, the results of this thesis show that by using multi-dimensional NMR exchange and correlation methods, one can exploit the susceptibility induced inhomogeneous magnetic field present in porous materials to obtain information about the pore size and heterogeneities of the system. The use of software for both visualizing the internal field and gradient and for simulating our experiment has proved to be invaluable for this work. It is hoped that the methods presented here could be useful as another building block in the study of porous media with NMR. 


\section{Appendix A}

\section{Publications from this Thesis}

1. L.M. Burcaw and P.T. Callaghan "Multidimensional Correlation and Exchange Experiments Using Local Fields in Porous Media" AIP Conf. Proc., December 5, 2008 1081:11-13 MAGNETIC RESONANCE IN POROUS MEDIA: Proceedings of the 9th International Bologna Conference on Magnetic Resonance in Porous Media (MRPM9), including 8th Colloquium on Mobile Magnetic Resonance (CMMR8)

2. L.M. Burcaw and P.T. Callaghan "Observation of molecular migration in porous media using 2D exchange spectroscopy in the inhomogeneous magnetic field" Journal of Magnetic Resonance 198:167-173(2009)

3. L.M. Burcaw, M.W. Hunter, and P.T. Callaghan "Propagator-resolved 2D exchange in porous media in the inhomogeneous magnetic field" Journal of Magnetic Resonance 205:209-215(2010)

4. L.M Burcaw and P.T. Callaghan "Correlation of the inhomogeneous field as a measure of sample heterogeneity" In progress. 


\section{Bibliography}

[1] F. Bloch. Nuclear induction. Physical Review, 70:460-474, 1946.

[2] E.M. Purcell, H.C. Torrey, and R.V. Pound. Resonance absorption by nuclear magnetic moments in a solid. Physical Review, 69:37-38, 1946.

[3] Paul T. Callaghan. Principles of Nuclear Magnetic Resonance Microscopy. Oxford University Press, 1991.

[4] P.T. Callaghan. Translational Dynamics and Magnetic Resonance Principles of Pulsed Field Gradient NMR. Oxford University Press, In Press.

[5] Malcolm H. Levitt. Spin Dynamics. John Wiley and Sons, Ltd, 2008.

[6] James Keeler. Understanding NMR Spectroscopy. John Wiley and Sons, Ltd, 2005.

[7] Edwin D. Becker. High Resolution NMR. Academic Press, 2000.

[8] Frederick Reif. Fundamentals of Statistical and Thermal Physics. McGraw Hill Book Company, 1965.

[9] H.J. Weber and G.B. Arfken. Essential Mathematical Methods for Physicists. Elsevier Acadmic Press, 2004.

[10] I. Fredholm. Sur une classe d'Équations fonctionnelles. Acta Mathematica, 27:365-390, 1903.

[11] K.P. Whittall and A.L. MacKay. Quantitative interpretation of nmr relaxation data. Journal of Magnetic Resonancea, 84:134-152, 1989. 
[12] E.J. Fordham, A. Sezginer, and L.D. Hall. Imaging multiexponential relaxation in the $\left(y, \log _{e} t_{1}\right)$ plane, with application to clay filtration in rock cores. Journal of Magnetic Resonance, 113:139-150, 1995.

[13] D.L. Phillips. A technique for the numerical solution of certain integral equations of the first kind. Journal of the ACM, 9:84-97, 1962.

[14] Paul H. Merz. Determination of adsorption energy distribution by regularization and a characterization of certain adsorption isotherms. Journal of Computational Physics, 38:64-85, 1980.

[15] W. Provencher. A constrained regularization method for inverting data represented by linear algebraic or integral equation. Computer Physics Communications, 27:213-227, 1982.

[16] W. Provencher. Contin: A general purpose constrained regularization program for inverting noisy linear algebraic and integral equations. Computer Physics Communications, 27:229 - 242, 1982.

[17] A.N. Tychonoff and V.Y. Arsenin. Solution of Ill-Posed Problems. Winston and Sons, 1977.

[18] R.L. Vold and J.S. Waugh. Measurement of spin relaxation in complex systems. Journal of Chemical Physics, 48:3831-3832, 1968.

[19] E. L. Hahn. Spin echoes. Physical Review, 80(4):580, 1950.

[20] H.Y. Carr and E.M. Purcell. Effects of diffusion on free precession in nuclear magnetic resonance experiments. Physical Review, 94(3):630-638, 1954.

[21] S. Meiboom and D. Gill. Modified spin-echo method for measuring nuclear relaxation times. Review of Scientific Instruments, 29(8):688-691, 1958.

[22] A.G. Anderson, R.L. Garwin, E. L. Hahn, J.W. Horton, G.L. Tucker, and R.M. Walker. Spin echo serial storage memory. Journal of Applied Physics, 26:1324-1338, 1955. 
[23] D.W. McCall, D.C. Douglass, and E.W. Anderson. Self-diffusion studies by means of nuclear magnetic resonance spin-echo techniques. Ber. Bunsen-Ges. Phys. Chem., 67:336-340, 1963.

[24] E.O. Stejskal and J.E. Tanner. Spin diffusion measurements: Spin echoes in the presence of a time-dependent field gradient. Journal of Chemical Physics, 42(1):288-292, 1965.

[25] H.C. Torrey. Bloch equations with diffusion terms. Physical Review, 104(3):563 - 565, 1956.

[26] J.E. Tanner. Use of the stimulated echo in $\mathrm{nmr}$ diffusion studies. Journal of Chemical Physics, 52(5):2523-2526, 1970.

[27] K.J. Packer, C. Rees, and D.J. Tomlinson. A modification of the pulsed magnetic field-gradient spin echo method of studying diffusion. Molecular Physics, 18:421-423, 1970.

[28] W. David Williams, E.F.W. Seymour, and R.M. Cotts. A pulsed-gradient multiple-spin-echo $\mathrm{nmr}$ technique for measuring diffusion in the presence of background magnetic field gradients. Journal of Magnetic Resonance, 31:271-282, 1978.

[29] R.F. Karlicek and I.J. Lowe. A modified pulsed gradient technique for measuring diffusion in the presence of large background gradients. Journal of Magnetic Resonance, 37:75-91, 1980.

[30] R.M. Cotts, M.J.R. Hoch, T. Sun, and J.T. Markert. Pulsed field gradient stimulated echo methods for improved nmr diffusion measurements in heterogeneous systems. Journal of Magnetic Resonance, 83:252-266, 1989.

[31] J. Kärger and W. Heink. The propagator representation of molecular transport in microporous crystallites. Journal of Magnetic Resonance, 51:1-7, 1983.

[32] J. Jeener. Ampere International Summer School, 1971. 
[33] W.P. Aue, E. Bartholdi, and R. R. Ernst. Two-dimensional spectroscopy. application to nuclear magnetic resonance. Journal of Chemical Physics, 64(5):2229-2246, 1976.

[34] R. R. Ernst, G. Bodenhausen, and A. Wokaun. Principles of Nuclear Magnetic Resonance in One and Two Dimensions. Oxford University Press, 2003.

[35] J. Jeener, B. H. Meier, P. Bachmann, and R. R. Ernst. Investigation of exchange processes by two-dimensional nmr spectroscopy. Journal of Chemical Physics, 71:4546-4553, 1979.

[36] J.H. Lee, C. Labadie, C.S. Springer, and G.S. Harbison. Two-dimensional inverse laplace transform nmr: Altered relaxation times allow detection of exchange correlation. Journal of the American Chemical Society, 115(17):7761-7764, 1993.

[37] P.J. McDonald, J.-P. Korb, J. Mitchell, and L. Monteilhet. Surface relaxation and chemical exchange in hydrating cement pastes: A two-dimensional nmr relaxation study. Physical Review E, 72:011409, 2005.

[38] L. Monteilhet, J.-P. Korb, J. Mitchell, and P.J. McDonald. Observation of exchange of micropore water in cement pastes by two-dimensional $\mathrm{t} 2$ - $\mathrm{t} 2$ nuclear magnetic resonance relaxometry. Physical Review E, 2006.

[39] K. E. Washburn and P. T. Callaghan. Tracking pore to pore exchange using relaxation exchange spectroscopy. Physical Review Letters, 97(17):175502/1-4, October 2006.

[40] J-F. Kuntz, P. Palmas, V. Level, and D. Canet. Restricted diffusion and exchange of water in porous media: Average structure determination and size distribution resolved from effect of local field gradients on the proton nmr spectrum. Journal of Magnetic Resonance, 191:239-247, 2008.

[41] P. T. Callaghan, S. Godefroy, and B. N. Ryland. Use of the second dimension in pgse nmr studies of porous media. Magnetic Resonance Imaging, 21(3-4):243-248, April 2003. 
[42] P. T. Callaghan and I. Furo. Diffusion-diffusion correlation and exchange as a signature for local order and dynamics. Journal of Chemical Physics, 120(8):4032-4038, February 2004.

[43] P. L. Hubbard, K. M. McGrath, and P. T. Callaghan. A study of anisotropic water self-diffusion and defects in the lamellar mesophase. Langmuir, 21(10):4340-4346, May 2005.

[44] P. L. Hubbard, K. M. McGrath, and P. T. Callaghan. Orientational anisotropy in the polydomain lamellar phase of a lyotropic liquid crystal. Langmuir, 22(9):3999-4003, April 2006.

[45] Y. Qiao, P. Galvosas, T. Adalsteinsson, M. Schonhoff, and P. T. Callaghan. Diffusion exchange nmr spectroscopic study of dextran exchange through polyelectrolyte multilayer capsules. Journal Of Chemical Physics, 122(21):214912, June 2005.

[46] Y. Qiao, P. Galvosas, and P. T. Callaghan. Diffusion correlation nmr spectroscopic study of anisotropic diffusion of water in plant tissues. Biophysical Journal, 89(4):2899-2905, October 2005.

[47] M. D. Hurlimann, L. Venkataramanan, and C. Flaum. The diffusion-spin relaxation time distribution function as an experimental probe to characterize fluid mixtures in porous media. Journal of Chemical Physics, pages 10223-10232, 2002.

[48] H. Peemoeller and M.M. Pintar. Two-dimensional time-evolution approach for resolving a composite free-induction decay. Journal of Magnetic Resonance, pages 358-360, 1980.

[49] H. Peemoeller, R.K. Shenoy, and M.M. Pintar. Two-dimensional nmr time evolution correlation spectroscopy in wet lysozyme. Journal of Magnetic Resonance, pages 193-204, 1981.

[50] A.E. English, K.P. Whittall, M.L.G. Joy, and R.M. Henkelman. Quantitative two-dimensional time correlation relaxometry. Magnetic Resonance in Medicine, pages 425-434, 1991. 
[51] Y. Q. Song, L. Venkataramanan, M. D. Hurlimann, M. Flaum, P. Frulla, and C. Straley. $t_{1}-t_{2}$ correlation spectra obtained using a fast two-dimensional laplace inversion. Journal Of Magnetic Resonance, 154(2):261-268, February 2002.

[52] F.A.L. Dullien. Porous Media Fluid Transport and Pore Structure. Academic Press, 1992.

[53] P.J. Barrie. Characterization of porous media using nmr methods. Annual Reports on NMR Spectroscopy, 41:265-278, 2000.

[54] Jacob Bear. Dynamics of Fluids in Porous Media. Dover Publications, Inc, 1988.

[55] A.T. Watson and C.T. Chang. Characterizing porous media with nmr methods. Progress in Nuclear Magnetic Resonance Spectroscopy, 31:343-386, 1997.

[56] J.R. Banavar and L.M. Schwartz. Magnetic resonance as a probe of porous media. Physical Review Letters, 58:1411-1414, 1987.

[57] G.E. Archie. The electrical resistivity log as an aid in determining some reservoir characteristics. Transactions of the American Institute of Mining and Metallurgical Engineers, 146:54-61, 1942.

[58] P.N. Sen, L.M. Schwartz, P.P. Mitra, and B.I. Halperin. Surface relaxation and the long-time diffusion coefficient in porous media: Periodic geometries. Physical Review B, 49:215-225, 1994.

[59] R.J. Brown and I. Fatt. Measurement of fractional wettability on oil field rocks by the nmr method. Petroleum Transactions of AIME, 207:262-264, 1956.

[60] J.R. Zimmerman and W.E. Brittin. Nuclear magnetic resonance studies in multiple phase systems: Lifetime of a water molecule in an adsorbing phase on silica gel. Journal of Physical Chemistry, 61:1328-1333, 1957. 
[61] S.D. Senturia and J.D. Robinson. Nuclear spin-lattice relaxation of liquids confined in porous solids. SPE Journal, 10:237-244, 1970.

[62] K.R. Brownstein and C.E. Tarr. Spin-lattice relaxation in a system governed by diffusion. Journal of Magnetic Resonance, 26:17-24, 1977.

[63] R.L. Kleinberg, W.E. Kenyon, and P.P. Mitra. Mechanism of nmr relaxation of fluids in rock. Journal of Magnetic Resonance, Series A, 108:206-214, 1994.

[64] G.C. Borgia, R.J.S. Brown, and P. Fantazzini. Nuclear magnetic resonance relaxivity and surface-to-volume ratio in porous media with a wide distribution of pore sizes. Journal Of Applied Physics, 79:3656-3664, 1996.

[65] M. D. Hurlimann, K. G. Helmer, L. L. Latour, and C. H. Sotak. Restricted diffusion in sedimentary rocks. determination of surface-area-tovolume ratio and surface relaxivity. Journal of Magnetic Resonance, Series A, 111(2):169 - 178, 1994.

[66] H-K Liaw, R. Kulkarni, S. Chen, and A.T. Watson. Characterization of fluid distributions in porous media by $\mathrm{nmr}$ techniques. AIChE, 42(2):538546, 1996.

[67] M. Lipsicas, J.R. Banavar, and J. Willemsen. Surface relaxation and pore sizes in rocks - a nuclear magnetic resonance analysis. Applied Physics Letters, 48:1544-1546, 1986.

[68] R.L. Kleinberg and M.A. Horsfield. Transverse relaxation processes in porous sedimentary rock. Journal of Magnetic Resonance, 88:9-19, 1990.

[69] P.P. Mitra, P.N. Sen, L.M. Schwartz, and P. Le Doussal. Diffusion propagator as a probe of the structure of porous media. Physical Review Letters, 68:3555-3558, 1992.

[70] R. J. Brown. Distribution of fields from randomly placed dipoles - freeprecession signal decay as result of magnetic grains. Physical Review, 121(5):1379-1382, 1961. 
[71] L. E. Drain. Broadening of magnetic resonance lines due to field inhomogeneities in powdered samples. Proceedings Of The Physical Society Of London, 80(518):1380, 1962.

[72] P. N. Sen and S. Axelrod. Inhomogeneity in local magnetic field due to susceptibility contrast. Journal Of Applied Physics, 86(8):4548-4554, 1999.

[73] H. Cho, S. Ryu, J.L. Ackerman, and Y.-Q. Song. Visualization of inhomogeneous local magnetic field gradient due to susceptibility contrast. Journal of Magnetic Resonance, 198:88-93, 2009.

[74] B. Audoly, P.N. Sen, S. Ryu, and Y-Q Song. Correlation functions for inhomogeneous magnetic field in random media with application to a dense random pack of spheres. Journal of Magnetic Resonance, 164:154-159, 2003.

[75] Q. Chen, A. E. Marble, B. G. Colpitts, and B. J. Balcom. The internal magnetic field distribution, and single exponential magnetic resonance free induction decay, in rocks. Journal Of Magnetic Resonance, 175(2):300-308, 2005.

[76] P.W. Anderson and P.R. Weiss. Exchange narrowing in paramagnetic resonance. Reviews of Modern Physics, 25:269-276, 1953.

[77] C.F. Hazlewood, D.C. Chang, B.L. Nichols, and D.E. Woessner. Nuclear magnetic resonance transverse relaxation times of water protons in skeletal muscle. Biophysical Journal, 14:583-606, 1974.

[78] Y-Q Song, S. Ryu, and P. N. Sen. Determining multiple length scales in rocks. Nature, 406(6792):178 - 181, July 2000.

[79] H. Cho and Y-Q Song. Nmr measurement of magnetic field correlation function in porous media. Physical Review Letters, 100:025501, 2008.

[80] M. Winkler, M. Zhou, M. Bernardo, B. Endeward, and H. Thomann. Internal magnetic gradient fields in glass bead packs from numerical simulations and constant time diffusion spin echo measurements. Magnetic Resonance Imaging, pages 311-315, 2003. 
[81] M. D. Hurlimann. Effective gradients in porous media due to susceptibility differences. Journal Of Magnetic Resonance, 131(2):232-240, 1998.

[82] A. Leventis, G. Papavassiliou, M. Fardis, and F. Milia. 1h nmr study of the local magnetic field gradients in evoluting porous structures. an application to cement gels. Journal of Chemical Physics, 113(17):7621 - 7626, November 2000.

[83] B. Sun and K. J. Dunn. Probing the internal field gradients of porous media. Physical Review E, 65(5):051309/1-7, May 2002.

[84] D.E. Woessner. Nmr spin-echo self-diffusion measurements on fluids undergoing restricted diffusion. Journal of Physical Chemistry, 67:1365-1367, 1962.

[85] P. N. Sen. Time-dependent diffusion coefficient as a probe of geometry. Concepts in Magnetic Resonance A, 23A(1):1-21, 2004.

[86] Albert Einstein. Investigations on the Theory of the Brownian Movement. Dover Publications, Inc, 1956.

[87] P.P. Mitra, P. N. Sen, and L.M. Schwartz. Short-time behavior of the diffusion coefficient as a geometrical probe of porous media. Physical Review B, 47(14):8565 - 8574, 1993.

[88] T.M. de Swiet and P.N. Sen. Time dependent diffusion coefficient in a disordered medium. Journal of Chemical Physics, 104:206-209, 1996.

[89] G.A. Baker and P. Graves-Morris. Pade Approximants. Cambridge University Press, 1996.

[90] L.L. Latour, P.P. Mitra, R.L. Kleinberg, and C.H. Sotak. Time-dependent diffusion coefficient of fluids in porous media as a probe of surface-tovolume ratio. Journal of Magnetic Resonance, Series A, 101:342-346, 1993.

[91] R.W. Mair, P.N. Sen, M. D. Hurlimann, S. Patz, D.G. Cory, and R. L. Walsworth. The narrow pulse approximation and long length scale de- 
termination in xenon gas diffusion $\mathrm{nmr}$ studies of model porous media. Journal of Magnetic Resonance, 156:202-212, 2002.

[92] J.E. Tanner. Transient diffusion in a sysetm partitioned by permeable barriers. application to $\mathrm{nmr}$ measurements with a pulsed field gradient. Journal of Chemical Physics, 69:1748-1754, 1978.

[93] R.N. Bracewell. The Fourier Transform and its Applications. McGraw Hill Book Company, 2000.

[94] D. G. Cory and A.N. Garroway. Measurement of translational displacement probabilities by nmr: An indicator of compartmentation. Magnetic Resonance in Medicine, 14:435-444, 1990.

[95] P.T. Callaghan, D. MacGowan, K.J. Packer, and F.O. Zelaya. Highresolution q-space imaging in porous structures. Journal of Magnetic Resonance, 90:177-182, 1990.

[96] A.N. Jackson, M. W. Hunter, and P.T. Callaghan. Simulating dispersion in porous media. 2009.

[97] J.D. Jackson. Classical Electrodynamics, 3rd Edition. John Wiley and Sons, Inc, 1999.

[98] K.R. Brownstein and C.E. Tarr. Importance of classical diffusion in nmr studies of water in biological cells. Physical Review A, 19:2446-2453, 1979.

[99] M. W. Hunter. Personal Communication, 2011.

[100] L. Venkataramanan, Y. Q. Song, and M. D. Hurlimann. Solving fredholm integrals of the first kind with tensor product structure in 2 and 2.5 dimensions. IEEE Transactions On Signal Processing, 50(5):1017-1026, May 2002.

[101] M. D. Hurlimann and L. Venkataramanan. Quantitative measurement of two-dimensional distribution functions of diffusion and relaxation in grossly inhomogeneous fields. Journal of Magnetic Resonance, 157:31-42, 2002. 
[102] J. G. Seland, K. E. Washburn, H. W. Anthonsen, and J. Krane. Correlations between diffusion, internal magnetic field gradients, and transverse relaxation in porous systems containing oil and water. Physical Review E, 70(5):051305/1-10, November 2004.

[103] S. Godefroy and P. T. Callaghan. 2d relaxation/diffusion correlations in porous media. Magnetic Resonance Imaging, 21(3-4):381-383, April 2003.

[104] K. E. Washburn and P. T. Callaghan. Propagator resolved transverse relaxation exchange spectroscopy. Journal Of Magnetic Resonance, 186(2):337340, June 2007.

[105] K.E. Washburn, C. H. Arns, and P.T. Callaghan. Pore characterization through propagator-resolved transverse relaxation exchange. Physical Review E, page 051203, 2008.

[106] A Coy and P. T. Callaghan. Pulsed gradient spin-echo nmr "diffusive diffraction" experiments on water surrounding close-packed polymer spheres. Journal of Colloid and Interface Science, 168:373-379, 1994.

[107] S. Stapf. Nmr investigations of correlations between longitudinal and transverse displacements in flow through random structured media. Chemical Physics, 284:369-388, 2002.

[108] K.F. Morris and C.S. Johnson. Resolution of discrete and continuous molecular size distributions by means of diffusion-ordered $2 \mathrm{~d}$ nmr spectroscopy. Journal of the American Chemical Society, 115:4291-4299, 1993.

[109] P. Stilbs, K. Paulsen, and P.C. Griffiths. Global least-squares analysis of large, correlated spectral data sets: Application to component-resolved ftpgse nmr spectroscopy. Journal of Physical Chemistry, 100:8180-8189, 1996.

[110] C.H. Arns, T. AlGhamdi, and J-Y. Arns. Numerical analysis of nmr relaxation-diffusion responses of sedimentary rock. 